\title{
WestVirginiaUniversity
}

THE RESEARCH REPOSITORY @ WVU

Graduate Theses, Dissertations, and Problem Reports

2018

\section{Automated Analysis of Polypharmacy using Prescription Drug Claims Data}

Shahad Nagoor

Follow this and additional works at: https://researchrepository.wvu.edu/etd

\section{Recommended Citation}

Nagoor, Shahad, "Automated Analysis of Polypharmacy using Prescription Drug Claims Data" (2018). Graduate Theses, Dissertations, and Problem Reports. 7219.

https://researchrepository.wvu.edu/etd/7219

This Thesis is protected by copyright and/or related rights. It has been brought to you by the The Research Repository @ WVU with permission from the rights-holder(s). You are free to use this Thesis in any way that is permitted by the copyright and related rights legislation that applies to your use. For other uses you must obtain permission from the rights-holder(s) directly, unless additional rights are indicated by a Creative Commons license in the record and/ or on the work itself. This Thesis has been accepted for inclusion in WVU Graduate Theses, Dissertations, and Problem Reports collection by an authorized administrator of The Research Repository @ WVU. For more information, please contact researchrepository@mail.wvu.edu. 


\title{
Automated Analysis of Polypharmacy using Prescription Drug Claims Data
}

\author{
Shahad Nagoor \\ Thesis submitted to the \\ Benjamin M. Statler College of Engineering and Mineral Resources \\ at West Virginia University \\ in partial fulfillment of the requirements \\ for the degree of
}

Master of Science

in

Computer Science

Donald A. Adjeroh, Ph.D., Chair

Sambamoorthi Usha, Ph.D.

YanFang Ye, Ph.D.

Savage Saiph, Ph.D.

Lane Department of Computer Science and Electrical Engineering

West Virginia University

Morgantown, West Virginia

May 2018

Keywords: polypharmacy analysis, multiple use of medications, inappropriate medications, prescriptions claim examination, emergency room admissions, drug ingredients

Copyright 2018 Shahad Nagoor 


\title{
Abstract \\ Automated Analysis of Polypharmacy using Prescription Drugs Claims Data
}

\author{
Shahad Nagoor
}

Patients are often prescribed with multiple medications, especially those with chronic conditions. This leads to the possibility of concurrent use of multiple medications in overlapping time intervals. Clinical studies have shown that polypharmacy has a substantial impact on health outcomes. Increasing the number of medications that an individual or a patient takes increases the likelihood that the individual or patient would have an adverse drug event, drug misuse, drug overdose, or reduced therapeutic effect of the drugs. These problems are often behind emergency room admissions in hospitals. Thus, studying polypharmacy problems and ways to resolve them have long been of great interest to health care professionals.

In this thesis, we focus on analyzing polypharmacy based on information extracted from prescription drug claims data. Prior work has defined polypharmacy, based mainly on the number of drugs, or the number of drug classes. Here, we emphasize the use of drug active ingredients as another basis for definition of polypharmacy. Then, we develop an algorithm for automated determination of the thresholds required for each of the different definitions of polypharmacy. Based on this, we perform a comparative analysis of the different approaches to polypharmacy. We analyzed the possible association between levels of polypharmacy and ER visits. Independent of the basis used for the polypharmacy definition, the results showed a general increase in ER problems with increasing number of drugs/drug classes/or active ingredients. However, the threshold for polypharmacy varied with the specific basis used in the definition. This observation led to a composite definition of polypharmacy, involving the use of a combination of different basic elements at the same time, rather than the current practice of using one single element. Further computational analysis included studying the occurrence of single-elements (e.g., single drugs, single classes, or single active ingredients), and the co-occurrence of multiple elements of the same basic type (e.g., two-drugs, two drug classes, two active ingredients, three-drugs, three drug classes, three active ingredients, etc.). This provides a repository of incidences of common drugs, active ingredients, drug class polypharmacy combinations, along with their richness, which can be a basis for further studies in drug safety and drug efficacy.

The results from this work provides a framework for healthcare specialists to analyze the impact of each polypharmacy definition and their respective associations with emergency room (ER) visits, and which combinations of drugs, of drug classes, or of active ingredients are likely to result in significant hospitalization, and thus should be avoided. 


\section{Dedication}

To my husband 'Foad', my son 'Mohammed', my daughter 'Danah' and our parents, sisters and brothers. 


\section{Acknowledgements}

I would like to express sincere gratitude to my research advisor Dr. Donald A. Adjeroh, for his insightful guidance. I am thankful for him for understanding my condition and being extremely supportive for my work. His advice continuously contributed to making this project better. His earnest endeavor imparted his best thoughts to my work. I also thank my committee members, Dr. Usha Sambamoorthi for her effort in preparing the data for this project, Dr.Saiph Savage and Dr. YanFang Ye for their knowledgeable suggestions. My deepest gratitude and love to my husband Dr.Foad Bukhari for his pure love, continues support, endless patience and for his medical consultations supporting my work. I am grateful and blessed to have my kids Muhammed and Danah who have been my strength and joy in my tough times. I would like to express my deep appreciation for my mother Amal Alhindi, my father Hussain Nagoor, my husbund's parents: Sabria Banafe'e and Asaad Bukhari for their unconditional love, kind words and for all of their sincere prayers. I am blessed with sisters and brothers who were helpful by their respective capacities and encouragements that made this master's journey easier for me. Finally, I express my obedience and gratitude to my God, my creator, and sustainer Allah, for illuminating the road ahead my dreams. 


\section{TABLE OF CONTENTS}

TABLE OF CONTENTS

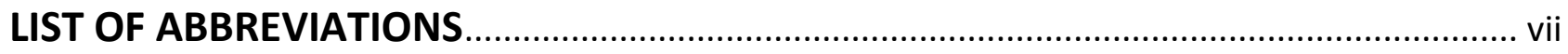

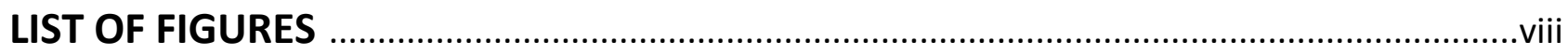

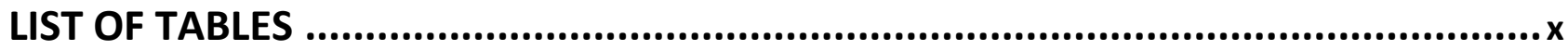

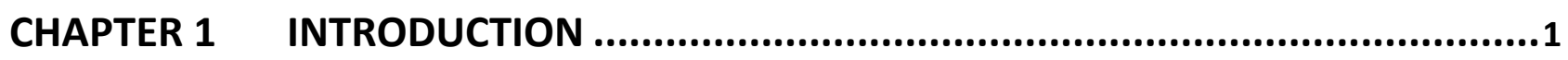

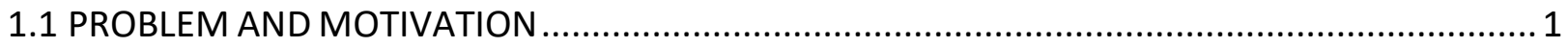

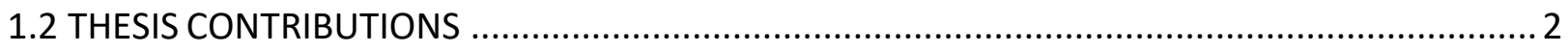

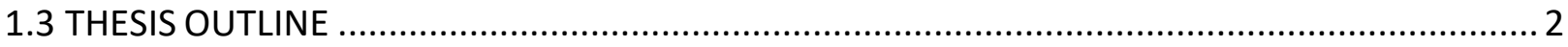

CHAPTER 2 BACKGROUND AND RELATED WORK ...................................... 3

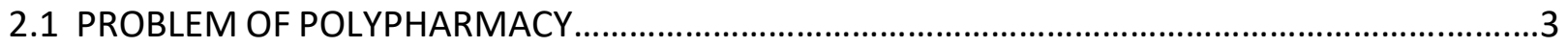

2.2 DEFINITIONS OF POLYPHARMACY .............................................................................

2.3 POLYPHARMACY -- THE GOOD AND THE BAD ......................................................................

2.4 METHODS OF DETECTION AND TYPES OF DATA USED ..........................................................

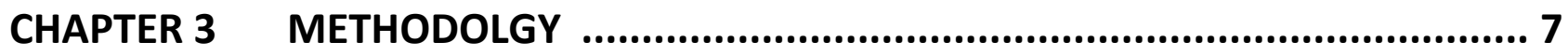

3.1 THREE DEFINTIONS OF POLYPHARMACY....................................................................... 7

3.1.1 Defining polypharmacy using number of drugs.......................................................... 7

3.1.2 Defining polypharmacy using number of drug classes............................................... 7

3.1.3 Defining polypharmacy using active ingredients .................................................... 9

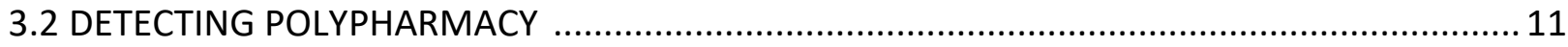

3.3 AUTOMATED THRESHOLD FOR POLYPHARMACY ...............................................................14

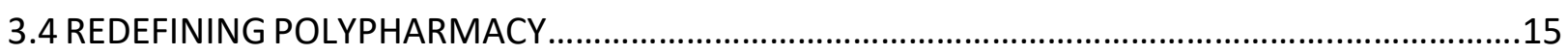

3.5 COMPUTATIONAL ANALYSIS FOR THE THREE DEFINTIONS....................................................16

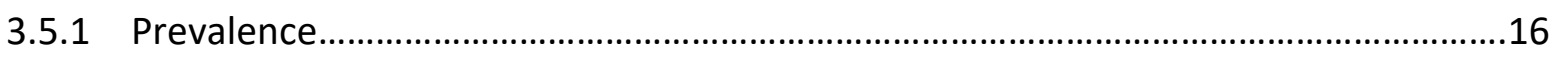

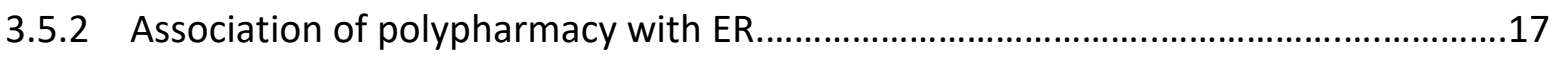

3.5.3 Richness and over-representations .........................................................................18

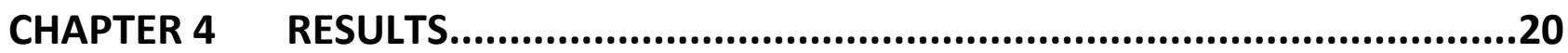

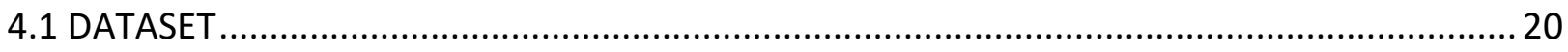

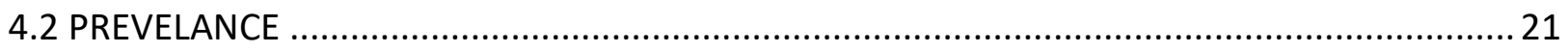


4.2.1 Prevalence of polypharmacy using number of drugs ......................................... 21

4.2.2 Prevalence of polypharmacy using number of Active ingredients ......................... 25

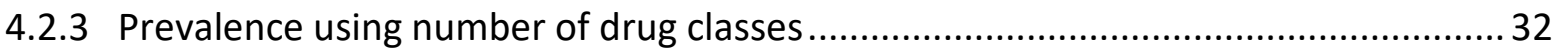

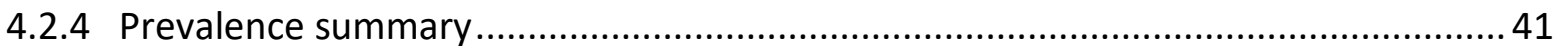

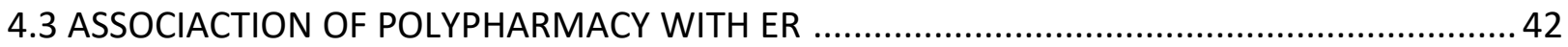

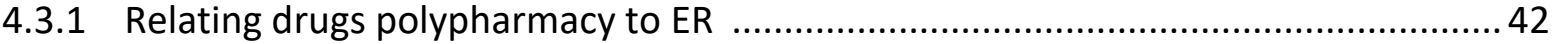

4.3.2 Relating active ingredients polypharmacy to ER ............................................ 45

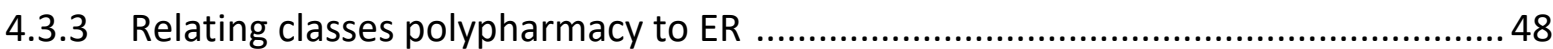

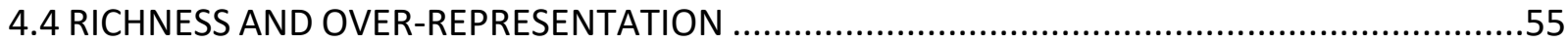

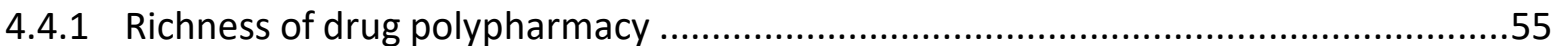

4.4.2 Richness of active ingredients polypharmacy ................................................60

4.4.3 Richness of drug classes polypharmacy.........................................................66

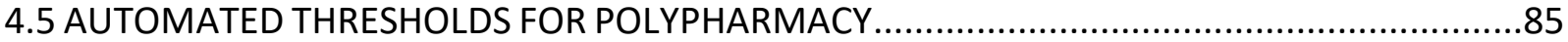

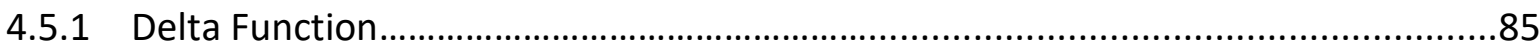

4.5.2 Choosing Polypharmacy Thresholds ....................................................................91

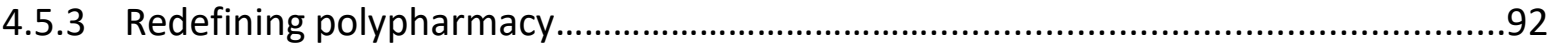

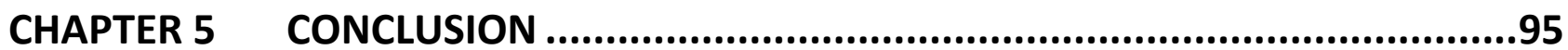

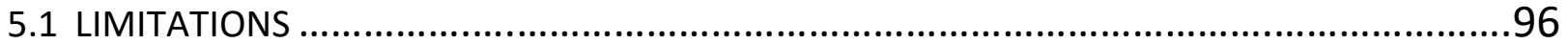

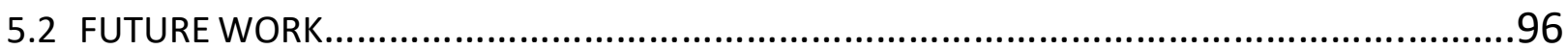

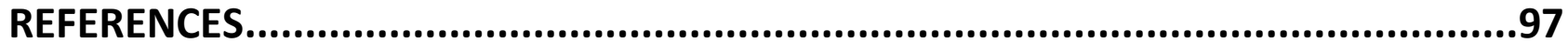

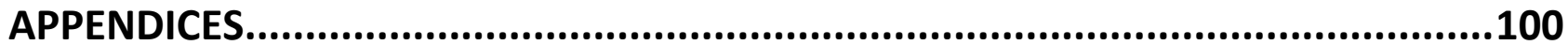

A TC1S1 Class Codes and Names....................................................................................100

B Associations of three-element polypharmacy with ER ..........................................................104

C Richness of three-element polypharmacy..............................................................................121 


\section{LIST OF ABBREVIATIONS}

$\begin{array}{ll}\text { PP } & \text { Polypharmacy } \\ \text { ER } & \text { Emergency Room } \\ \text { Al } & \text { Active ingredients } \\ \text { TC1 } & \text { MULTUM THERAPEUTIC CLASS \#1 } \\ \text { TC1S1 } & \text { MULTUM THERAPEUTIC SUB-CLASS \#1 FOR TC1 }\end{array}$




\section{LIST OF FIGURES}

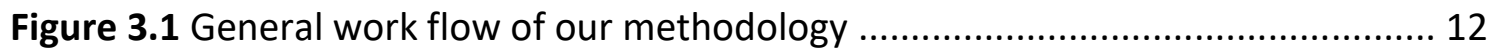

Figure 3.2 Polypharmacy detection algorithm ..................................................... 13

Figure 4.1 Ratio of ER visits to the number of polypharmacy for 5 polypharmacy groups based on the number of drugs. 44

Figure 4.2 Proportion of ER visits for given polypharmacy groups based on the number of drugs. 44

Figure 4.3 The probability that a patient in a given polypharmacy group will visit ER in a given year. 45

Figure 4.4 Ratio of ER visits to the number of polypharmacy for 5 polypharmacy groups based on the number of active ingredients.

Figure 4.5 Proportion of ER visits for given polypharmacy groups based on the number of active ingredients. 47

Figure 4.6 The probability that a patient in a given polypharmacy group will visit ER in a given year. .48

Figure 4.7 Ratio of ER visits to the number of polypharmacy for 5 polypharmacy groups based on the number of TC1 drug class. .50

Figure 4.8 Proportion of ER visits for given polypharmacy groups based on the number of TC1 drug class. .50

Figure 4.9 The probability that a patient in a given polypharmacy group will visit ER in a given year. 51

Figure 4.10 Ratio of ER visits to the number of polypharmacy for 5 polypharmacy groups based on the number of TC1S1 drug class.

Figure 4.11 Proportion of ER visits for given polypharmacy groups based on the number of TC1S1 drug class. .53

Figure 4.12 The probability that a patient in a given polypharmacy group will visit ER in a given year. .54 
Figure 4.13. drug pair occurrence richness plotted against drug pair ER occurrence richness in log scale

Figure 4.14. Relationship between active ingredients occurrence and ER richness using a log scale (logarithms are in base 10). 66

Figure 4.15. relationship between TC1 class pairs occurrence and ER richness. Logarithms are in base 10.

Figure 4.16. Relationship between TC1S1 class pairs occurrence and ER richness.

Logarithms are in base 10 . .77

Figure 4.17. Values of $\Delta(K, 1)$ for drug PP vs. ER measure for years (2011- 2015)..........86

Figure 4.18. Values of $\Delta(K, 1)$ for active ingredients PP vs. ER measure of years (20112015)

Figure 4.19. Values of $\Delta(K, 1)$ for TC1 class PP vs. ER measure of years (2011-2015......88

Figure 4.20. Values of $\Delta(K, 1)$ for TC1S1 class PP vs. ER measure for years (20112015) .89

Figure 4.21. Values of $\Delta(K, 1)$ for all PP vs. ER measure of years (2011-2015).............90

Figure 4.22. compares \#ER/\#PP for all possible definitions of polypharmacy. .93 


\section{LIST OF TABLES}

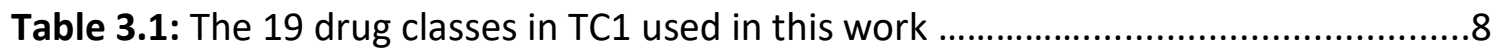

Table 4.1: Summary of MEPS dataset characteristics................................................ 20

Table 4.2: Statistics on prevalence of polypharmacy based on number of drugs.......... 21

Table 4.3: Top 30 single-drugs in the dataset ranked by their occurrences.................... 22

Table 4.4: Top 30 two-drug polypharmacy ranked by their occurrences........................ 23

Table 4.5: Top 30 three-drug polypharmacy ranked by their occurrences...................... 24

Table 4.6: Statistics on Prevalence of Polypharmacy using number of active

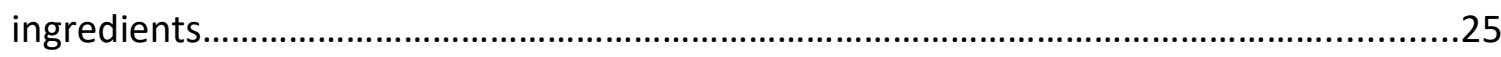

Table 4.7: Top 30 single-ingredients polypharmacy ranked by their occurrences...........26

Table 4.8: Top 30 two-active ingredient polypharmacy ranked by their occurrences.....27

Table 4.9: Top 30 three-active ingredient polypharmacy ranked by their occurrences..28

Table 4.10: List of ingredients/substances matched with their parent drugs..................29

Table 4.11: Prevalence of polypharmacy using number of drug classes .........................32

Table 4.12: Single TC1-class polypharmacy ranked by their occurrences .......................33

Table 4.13: Top 30 two TC1-class polypharmacy ranked by their occurrences...............34

Table 4.14: Top 30 three TC1-class polypharmacy ranked by their occurrences.............35

Table 4.15: Top 30 single TC1S1-classes in the dataset ranked by their occurrences......37

Table 4.16: Top 30 two-TC1S1 classes polypharmacy ranked by their occurrences.........38

Table 4.17: Top 30 three-TC1S1 classes polypharmacy ranked by their occurrences......39

Table 4.18: Summary of prevalence 2011 -2015.............................................................41

Table 4.19: Proportion of single-element, two-element, and three-element of polypharmacy of all definitions. 
Table 4.20: Three measures relating the number of drugs in polypharmacy groups to ER visits

Table 4.21: Three measures relating the number of active ingredients in polypharmacy to ER visits .46

Table 4.22: Three measures relating the number of TC1 classes in polypharmacy to ER visits

Table 4.23: Three measures relating the number of TC1S1 classes in polypharmacy to

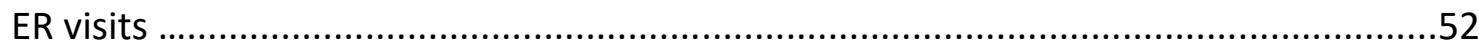

Table 4.24: Summary of average values for all definitions and for all years.................54

Table 4.25: Top 30 drug-pairs and their co-occurrences probability ranked by ER total..

Table 4.26: Individual and joint probability of occurrence for the top 30 two-drug polypharmacy (sorted by ER total) with richness of occurrence and ER .57

Table 4.27: Examples of drug pairs with high occurrence richness and low ER richness, high occurrence richness and low ER richness, and low occurrence richness and high ER richness.

Table 4.28: Top 30 active ingredient-pairs and their co-occurrences probability ranked by ER total.

Table 4.29: Individual and joint probability of occurrence for the top 30 two-active ingredient polypharmacy (sorted by ER total) with richness of occurrence and ER

Table 4.30: Comparison of some single drugs and their continent active ingredients ranking based on ER visits along with the richness of the active ingredient pairs.

Table 4.31: Examples of active ingredient pairs with high occurrence richness and low ER richness, high occurrence richness and low ER richness, and low occurrence richness and high ER richness.

Table 4.32: Top 30 TC1 class-pairs and their co-occurrences probability ranked by ER total. 
Table 4.33: Individual and joint probability of occurrence for the top 30 two-TC1 class polypharmacy (sorted by ER total) with richness of occurrence and ER

Table 4.34: Example of TC1 class pairs with high occurrence richness and low ER richness, high occurrence richness and low ER richness, and low occurrence richness and high ER richness.

Table 4.35: Summary of Top 30 TC1 class pairs and their richness with respect to occurrence and ER

Table 4.36: Top 30 TC1S1 class-pairs and their co-occurrences probability ranked by ER total. 74

Table 4.37: Individual and joint probability of occurrence for the top 30 two-TC1S1 class polypharmacy (sorted by ER total) with richness of occurrence and ER

Table 4.38: Examples of TC1S1 class pairs with high occurrence richness and low ER richness, high occurrence richness and low ER richness, and low occurrence richness and high ER richness.

Table 4.39: Comparison of some single TC1 classes and some of their continent TC1S1 class pairs ranking based on ER visits along with the richness of the TC1S1 class pairs.

Table 4.40: Two TC1 classes and their TC1S1 subclasses. .80

Table 4.41: Comparison of some TC1 class pairs and some of their continent TC1S1 class pairs ranking based on ER visits along with their richness values......

Table 4.42: Summary of ER richness occurrence for the top 20 polypharmacy using each definition ranked by \#ER visits.

Table 4.43: Values of $\Delta(K, 1)$ for drugs PP vs. ER measure for years (2011-2015).........86

Table 4.44: Values of $\Delta(K, 1)$ for active ingredient PP vs. ER measure for years (2011-2015)

Table 4.45: Values of $\Delta(K, 1)$ for TC1 class PP vs. ER measure of years (2011- 2015)....88

Table 4.46: Values of $\Delta(K, 1)$ for TC1S1 class PP vs. ER measure of years (2011- 2015). 89 
Table 4.47: Average values of $\Delta(K, 1)$ for all PP vs. ER measure of years (2011-2015)...90

Table 4.48: Summary statistics on the values for the delta function $(\Delta(K, 1))$, and

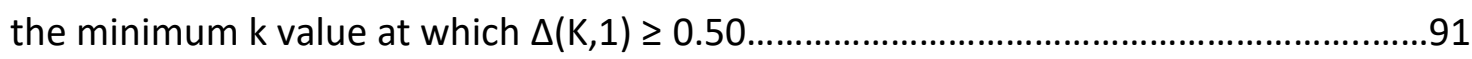

Table 4.49: Measuring \#ER/\#PP for all possible definitions of polypharmacy... .92 


\section{Chapter 1: Introduction}

\subsection{Problem and Motivation}

From daily supplements to life-saving medications, patients are likely to be using multiple medications including prescription drugs and over the counter medications. This is especially the case for those patients that suffer from chronic conditions. This could lead to the concomitant use of multiple medications in overlapping time intervals. Taking multiple medications at the same time has been described as polypharmacy.

Polypharmacy has no standard definition. It basically has been defined as using more than one medication but with no specific upper boundary. An example definition is the standard deviation above the mean number of medications [1]. Others used 2, 3, 5, 10 or more medications [2]. A study of polypharmacy in the U.S. showed that $10 \%$ of the population and $30 \%$ of older adults in the US take five or more drugs at the same time [3]. This is because each chronic disease often results in prescription of multiple medications based on current medical practice guidelines. "An elderly patient with at least two disease states, such as heart failure and chronic obstructive pulmonary disease, will usually exceed this arbitrary threshold of $>$ five medications "[2]. These guidelines have no strict monitoring requirement and thus patients could be subjected to over-prescription [3].

Studies have shown that polypharmacy has significant implications on peoples' health $[4,5]$. The term "Polypharmacy" is not necessarily negative per se. However, polypharmacy raises the likelihood for someone, especially those who take large number of medications, to have an unhealthy mixture of medications. In other words, polypharmacy increases the risk of adverse drug events, drug-interactions, medication non-adherence, decreased functional status and geriatric syndromes or, misuse, overuse, and underuse of those medications [6]. Thus, for some people, the term polypharmacy evokes more of the negative consequences. The more the number of drugs, the higher the risk of these problems [7].

In their book, Neel and Hogan [8] reported that based on studies, polypharmacy would be classified as the fifth leading cause of death in the United States. It is also shown that polypharmacy was behind $28 \%$ of all hospital admissions [8]. Morbidity and mortality in health care are highly associated with adverse drug reactions (ADRs). ADRs are responsible for more than 3.5 million physician office visits and 1 million emergency department visits each year [9]. The Institute of Medicine reported up to 98,000 annual deaths resulting from medical errors in 2000. 7,000 deaths among them were caused by ADRs $[2,10]$. In 2016, the overdose deaths were up to 63,600 [10]. 
Many emergency room cases involve such drug events. Unfortunately, and above all, polypharmacy also leads to increased health care costs for both the patient and the general healthcare system $[7,11]$. Thus, research have used different methods and techniques that contribute to optimize people's health by reducing polypharmacy with potential risk.

Although identifying polypharmacy and its potential risks has long been of interest to health care professionals, it has also been challenging, given the complexity and diversity of patients' data. Medical datasets are often huge, disparate, and more importantly, confidential. In addition to this, the uncertainty about the correct way to define the term "polypharmacy" places another challenge for recognizing and analyzing polypharmacy problems. Therefore, such analysis need to be enhanced by the use of automated algorithms for adjustable identification of the incidence of polypharmacy as well as estimating the potential risks for the patients involved.

\subsection{Thesis Contributions}

The contributions of this thesis are summarized as follows:

- We propose a new definition of polypharmacy using drug active ingredients as the basic element.

- We propose a new method for determining thresholds of polypharmacy using the number of drugs, active ingredients and drug classes. Based on the new thresholds, we propose a refinement to the definition of polypharmacy and analyze the impact of this refinement.

- We provide a detailed computational analysis of polypharmacy, providing a basis for comparative analysis of different definitions of polypharmacy on a popular dataset.

\subsection{Thesis Outline}

Chapter 2 provides a background on the problem of polypharmacy and a literature survey of relevant studies on polypharmacy with a brief discussion of their limitations. Chapter 3 introduces three definitions of polypharmacy, our methodology for detecting polypharmacy sets, and automated approach to determining polypharmacy thresholds. Chapter 4 presents the dataset used in this study and the results of our detailed statistical analysis of polypharmacy. Finally, Chapter 5 provides our overall conclusions as well as future work and recommendations. 


\section{Chapter 2: Background and Related Work}

\subsection{Problem of polypharmacy}

Studying polypharmacy problems have long been of great interest to health care professionals. Researchers have been looking for methods by which they can reliably evaluate the group of medications taken together to get answers about whether they are helpful, risky, or even unneeded. For this to be answered, an agreement on a standard way to define polypharmacy has to be reached in order to formalize what would be considered a polypharmacy and subsequently resolve its issues with higher consistency. Unfortunately, the definition of the term "polypharmacy" has been controversial topic in the health sciences literature.

According to the Viktil et al [12], the lack of a universal definition of polypharmacy makes it problematic to place effective measures to reduce it. This is because it depends on multiple factors such as the number of medications, the status of the patient's health, and the actual combination of medications being taken in that polypharmacy. Such measures should assist in avoiding inappropriate polypharmacy, but at the same time should not eliminate the appropriate multi-drugs that are to improve quality of live for some patients with chronic conditions. In particular, reducing polypharmacy should not lead to undertreatments. As a consequence of this, the threshold by which polypharmacy should be defined has remained unspecified, making the definition imprecise, and the line between useful and harmful polypharmacy very blury. Thus, studies of polypharmacy have used different definitions for the term "polypharmacy". Some studies examine polypharmacy issues by considering the number of certain elements involved in the group of medications. In fact, the specific number that constitute the polypharmacy is in turn debatable.

The multiple faces for polypharmacy also involve considering the type of data or medical element used as basis for its definition. The literature involves some studies that used the actual number of drugs $[1,2,4,7,17]$, and others that used the number of drug classes $[18,19,20]$. This makes a substantial difference for polypharmacy results. For instance, the polypharmacy of two or more drugs that are classified by the same drug class is not considered a polypharmacy by the definition of drug classes.

In addition to the problem of defining polypharmacy, predicting polypharmacy problems has been performed based on multiple variables. In particular, some studies use the number of drugs or drug classes as a leading predictor for problems. Others consider the actual combinations of drugs and drug classes. On the other hand, Jirón et al has shown that [Trends in prevalence and determinants of potentially inappropriate prescribing in the United States: 2007 to 2012]"The strongest predictor of PIM use was the number of drugs dispensed.", and Scott et al [Reducing Inappropriate Polypharmacy The Process of Deprescribing ] has concluded that "the 
single most important predictor of inappropriate prescribing and risk of adverse drug events in older patients is the number of prescribed drugs". Yet, according to the study [16], "numbers in polypharmacy are not the enemy, it is rather the ineffective, harmful, unnecessary manner of prescribing". Also, Kantor et al [14] pointed out that documenting the actual patterns of prescriptions is essential for both the clinical practitioners and researchers.

This also poses a conflict for researchers who study polypharmacy problems and makes defining polypharmacy challenging, ambiguous, and above all, necessary.

\subsection{Definitions of polypharmacy}

Kantor et al [14] consider a polypharmacy as 5 or more prescription drugs. Jyrkkä et al [15] classified polypharmacy into three groups, namely: non-polypharmacy group (0-5 drugs) polypharmacy (6 to 9 drugs), and excessive polypharmacy group (10 or more drugs).

In [12], Viktil et al reported that some studies used different classifications: minor polypharmacy with 2-4 drugs and major polypharmacy with 5 or more drugs. Others used: 2 or more drugs, 3 or more, 5 or more, 6 or more, 10 or more, but the most common number used to define polypharmacy was 5 . As reported in the literature, some studies used the actual drugs $[1,2,4,7,17]$, while others used drug classes $[18,19,20,21]$.

\subsection{Polypharmacy -- The Good and The Bad}

Polypharmacy could have positive or negative effects on patients. In [12] Viktil et al emphasized the janus face of polypharmacy and the importance of exploring the common recognition of the negative outcomes of polypharmacy. The janus face can be illustrated as avoiding the excessive use of drugs but also keeping the appropriate drugs that help reduce morbidity and are essential for improving the quality of life for people with multiple chronic conditions. Consequently, it is important to use the term polypharmacy with caution and not completely associate it with the negative consequences, as there could be necessary and useful polypharmacy.

Steinman [16] also pointed out the good aspect of some polypharmacy. According to his study, multidrug therapy can benefit older adults with multiple chronic diseases and are often considered necessary in managing such chronic conditions with their high level of comorbidity.

Jyrkkä et al [15] classified polypharmacy into three groups, namely: non-polypharmacy group (0-5 drugs) polypharmacy (6 to 9 drugs), and excessive polypharmacy group (10 or more drugs). In this study, interviews were done for elderly people of ages 75 and above in the first phase of analysis for data from 1998 to 2002. The second phase of analysis were conducted for survivors aged 80 and above in years 2003 to 2007. In their methodology, they performed advance regression to study the association that relates polypharmacy status and mortality. 
Their findings pointed out that the excessive polypharmacy is considered to be a main indicator for mortality in old people.

The study by Qato et al [13] performed a descriptive analysis for elderly of ages (62 to 85) in a nationaly representative sample in the U.S. Interviews were conducted in 2005-2006 and in 2010-2011 and polypharmacy were defined as taking at least 2 drugs at the regular use including prescriptions, over the counter, and dietary supplements. The extracted combinations were examined by Micromedex for drug-drug interaction examination. Their results showed an increase in the usage of interacting medications over time, and $15 \%$ of the subjects having the potential to be exposed to major interactions. Their analysis included prevalence of drugs and drug classes, and they estimated harmful interactions at the drug level.

Abe et al [24] conducted a study in Japan in which they attempted to connect polypharmacy results with emergency room visits. Statistical analysis on those visits showed that $69 \%$ of 3084 adult patients transported to hospital by ambulance, had polypharmacy. The mean number of their polypharmacy ranges from 3 to 6 . About $7 \%$ of patients had drug adverse events.

\subsection{Methods of detection and types of data used}

Maher et al [2] discussed the negative consequences of polypharmacy in Ambulatory Care, Hospital Setting, Nursing Home Setting and proved by evidence studies that intervention by inter-professional were effective in reducing unnecessary medications. Similar study was done by Zarowitz et al in 2012 [25]. Their objectives were to reduce polypharmacy risks, and cost. The intervention program involves clinical pharmacists. They performed a review for drug therapy and then contacted with physicians and patients to enhance their awareness about polypharmacy problems and solutions.

Rambhade et al conducted a study in India [6] that used a Questionnaire-based approach to analyze polypharmacy and its consequences. Patients were interviewed at pharmacy shops and their prescriptions were copied for the analysis. They found that $2.38 \%$ of the prescriptions showed over prescribing. 3.96\% showed repetition, while $2.39 \%$ showed drug-drug interactions. Then, they suggested a list of criteria that should be followed by prescribers for the sake of reducing inappropriate medications use.

In another study, Garfinkel et al [26], discussed and summarized the main international approaches to refine clinical and economic outcomes of inappropriate medication use and polypharmacy(IMUP) which involve clinical, pharmacological, computer-assisted and educational programs. By multiple studies reviews, it has been shown that using computer based prescription support system contributed in eliminating IMUP and severe drug-drug interactions.

Grando et al [27] used an ontology-based representation of drugs and their interactions

after reviewing existed drugs knowledgebase and built a system for assigning complexity to drug prescription using multiple factors such as dosage and frequency. This complexity was used later by a decision support system to estimate safety of polypharmacy prescriptions. 
Makar et al [28] used machine learning classifiers for prediction of six-month mortality in elderly people using Medicare claims data. They refined the features obtained from the best of traditional models and included not only the presence of diseases but also measures of their severity. This resulted in an augmented feature set that were tested using six classifiers. Their results outperformed traditional measures of mortality predictions that had been used in medical literature. This provides a prognostic tool that can be used in patient-physician decisions about end of life care and treatment options.

To sum up, prior work has studied multi-drug problems but never agreed on the exact definition of polypharmacy, the definite number for polypharmacy elements, the basic type of element used to define polypharmacy (i.e, drugs, drug-classes, etc). In this study, we investigate multiple definitions for polypharmacy derived from prior work in the literature and propose two more ways to define polypharmacy, the concomitant use of multiple medications. Next chapter introduces the definitions of polypharmacy and considers their advantages and limitations. This is followed by the methodology used in this work to analyze and compare the current and proposed definitions. 


\section{Chapter 3: Methodology}

\subsection{Three definitions of polypharmacy}

We start our analysis by first considering the different definitions of polypharmacy. In particular, we consider three definitions that differ in the key data type used as the basis for the definitions.

\subsubsection{Defining polypharmacy using number of drugs}

Defining polypharmacy using the number of drugs has been considered the classic and the most popular method used for studying polypharmacy. For instance, studies in $[1,2,4,7,17]$ used multi-drugs as their polypharmacy definition. Researchers have used this definition to look for harmful drug-drug interactions. This has been beneficial in recognizing risks at the drug level. However, the problems resulting from drugs could still be unspecified using this definition. In particular, this method may flag that using certain number or patterns of drugs as problematic but not provide sufficient justification on how could two or more drugs that are used together could interact negatively. In fact, this method also does not take into account potential problems with single drugs, as they are not considered as polypharmacy by the nature of this definition.

Thus, although this drug-based polypharmacy definition has provided useful recognition of some problems of using multiple-drug, there is still a need for a more detailed method by which the ambiguity or uncertainty behind single drug-related problems and even multiple-drug adverse reactions could be resolved and better explained. Investigating drug problem can be significantly enhanced by considering drug ingredients. Those ingredients may interact with each other, inhibit each other, enhance each other and therefore lead to misuse or overdose of certain substances. Although examining drugs ingredients should be intuitive, to our knowledge there is only one study prior to this work has considered the drug polypharmacy problem at the drug ingredients level. Section 3.1.3 will provide more detailed discussion about defining polypharmacy by drug ingredients.

\subsubsection{Defining polypharmacy using number of drug classes}

Medications are classified to groups using different criteria. They could be classified based on similarity of chemical structure, or the way they work, or the purpose for which they are used for.

For instance, Aspirin and sulfasalazine are classified as Salicylates based on similarity of their chemical structure. Aspirin is also classified as NSAID (Nonsteroidal anti-inflammatory drug). This class also contains Ibuprofen and Naproxen. Those medications are used for relieving mild to moderate pain such as mild joint inflammation and rheumatic fever. Also, Aspirin work on preventing blood clots, belonging to the class of medications called anti-platelets.

In general, patients should not be prescribed with two or more medications that share the same class since they may result in risks of overdose. Defining polypharmacy based on the 
drug classes has been used in the literature for beneficial monitoring of patients' medications prescriptions and examining their safety at the drug-class level. See $[18,19,20,21]$ for examples.

Researchers have used drug classes as the basic type to define polypharmacy because different classes may have different mechanisms of action. Based on this perspective, drugs that belong to the same class may not be considered as polypharmacy, but rather it would be considered as combinational therapy. Thus, this approach to define polypharmacy identifies the poly-mechanisms embedded in the prescribed drugs, which could not be captured by the definition of polypharmacy using the number of drugs.

A popular drug classification system is the Anatomical Therapeutic Chemical (ATC) classification systems. ATC classification system classifies drugs and their active ingredients based on the body organ or system that the drugs are expected to act on [22]. The therapeutic classes are defined according to the Multum/Lexicon therapeutic classification system [30]. In this work, we consider two therapeutic classes known as TC1 and TC1S1 for Therapeutic classes and Therapeutic sub-classes, respectively. TC1 assigns a drug to one or more therapeutic/chemical categories. Drugs can have up to three categories per drug while TC1S1 assigns one or more subcategories to a more general therapeutic class category given to a drug.

An example of these therapeutic classes is cardiovascular agents which include calcium channel blockers and diuretics sub classes. Likewise, central nervous system agents is a superclass for analgesics, anticonvulsants, and antiparkinson agents. Gastrointestinal agents contain the three subclasses: H2-receptor antagonists, antacids, antidiarrheals, and proton pump inhibitors. Metabolic agents cover antihyperlipidemic agents and antidiabetic agents. Psychotherapeutic agents include the subclass of antidepressants and antipsychotics. More information about these classifications and other types of classifications can be found at the Medical Expenditure Panel Survey website [29]. Table 3.1 shows the 19 drug classes in TC1 used in this work. Table 1 in Appendix A shows the 133 drug-sub classes in TC1S1. The relationship between TC1 and TC1S1 classes can be found at Multum Lexicon documentation [31].

Table 3.1 The 19 drug classes in TC1 used in this work.

\begin{tabular}{|c|l|}
\hline Class code & \multicolumn{1}{|c|}{ Class Name } \\
\hline-9 & Not ascertained \\
\hline 1 & Anti-infectives \\
\hline 19 & Antihyperlipidemic agents \\
\hline 20 & Antineoplastics \\
\hline 28 & Biologicals \\
\hline 40 & Cardiovascular agents \\
\hline 57 & Central nervous system agents \\
\hline 81 & Coagulation modifiers \\
\hline 87 & Gastrointestinal agents \\
\hline 97 & Hormones/hormone modifiers \\
\hline 105 & Miscellaneous agents \\
\hline 113 & Genitourinary tract agents \\
\hline 115 & Nutritional products \\
\hline
\end{tabular}




\begin{tabular}{|l|l|}
\hline 122 & Respiratory agents \\
\hline 133 & Topical agents \\
\hline 218 & Alternative medicines \\
\hline 242 & Psychotherapeutic agents \\
\hline 254 & Immunologic agents \\
\hline 331 & Radiologic agents \\
\hline 358 & Metabolic agents \\
\hline
\end{tabular}

Using drug classes for the purpose of recognizing polypharmacy issues can give a general idea about similar drug characteristics that are easier to remember for physicians and health care professionals. Yet it makes the association with health problems non-specific knowing that a class could include hundreds of drugs as members. Therefore, it could be misleading to rely on coprescribed drug-classes alone, since classes are composed of drugs that also work on multiple targets [23]. As indicated earlier in this section, these classes have been used to define polypharmacy in prior work. However, the problems of polypharmacy using drug classes have not been very well studied as compared with the corresponding use of number of drugs in analyzing polypharmacy. Thus, in our study we map drugs to drug classes and generate lists of drug class combinations. We then checked these combinations for association with the number of emergency room visits and compared the results with those of drugs and drugingredients.

\subsubsection{Defining polypharmacy using active ingredients}

Medications consists of multiple substances. These substances are classified as active ingredients and inactive ingredients or excipients. Inactive ingredients are used to stabilize medications and generally have no pharmacological effect. In particular, they often involve preservatives, and flavoring agents. On the other hand, active ingredients are those that have biological or pharmacological activity for treating a condition. As an example, consider the medication Childrens Tylenol Plus Multi-Symptom Cold. This medication contains four active

ingredients, namely, ACETAMINOPHEN, CHLORPHENIRAMINE MALEATE, DEXTROMETHORPHAN HYDROBROMIDE, and PHENYLEPHRINE HYDROCHLORIDE.

An instance for a polypharmacy defined on the basis of drugs is (ProColMycin, Symbyax, Nighttime Multi-Symptom Cold, Marcaine).

So, this polypharmacy includes four medications. On the other hand, the active ingredients polypharmacy resulting from these four medications contains 13 ingredients. They are listed below in the format: medication = [ active ingredients $A$; active ingredients $B$,...etc.].

ProCoMycin = [BACITRACIN; NEOMYCIN; POLYMYXIN B; LIDOCAINE HYDROCHLORIDE] 


\section{Symbyax $=$ [OLANZAPINE; FLUOXETINE HYDROCHLORIDE]}

\section{Nighttime Multi-Symptom Cold $=[$ ACETAMINOPHEN; CHLORPHENIRAMINE MALEATE; DEXTROMETHORPHAN HYDROBROMIDE; PHENYLEPHRINE]}

\section{Marcaine $=$ [BUPIVACAINE HYDROCHLORIDE ANHYDROUS; EPINEPHRINE BITARTRATE $]$}

Based on the above, and as discussed earlier in Section 3.1.1, it can be seen that ingredients-based polypharmacy can produce a more detailed list of substances that a patient consumes and thus safety and efficacy of polypharmacy can be studied using data inputs at a finer level of granularity.

In particular, considering polypharmacy by active ingratiates can expose potentially hidden patterns of risky (though unintentional) mix of substances resulting in possible unintentional overdose. Thus, defining polypharmacy using active ingredients can uncover the possibility for multiple substances taken, while embedded within one drug. The result is that even though one drug may not be considered as polypharmacy using the drug-based polypharmacy, it could be counted as polypharmacy when we use active ingredients as the basis of polypharmacy. However, it is also worthwhile to mention that getting the active ingredients of all existing drugs is challenging and not straightforward, given that there might be many ingredients with long and inconsistent names provided in the few datasets with this information, and these datasets are often incomplete.

To our knowledge, only one study [3] has used active ingredients in its definition of polypharmacy. Thus, our study is among the first studies that focus on establishing patterns of polypharmacy based on active ingredients and analyzing their impact on patients and their health outcomes. We use this definition of polypharmacy to compose a dataset of active ingredients combinations, and study their relationships with emergency room visits for the given patients. We compare the results of this definition of polypharmacy with the results of other methods discussed earlier. Comparing different definitions of polypharmacy can lead to answers to key questions about which method of defining polypharmacy could lead to improved discovery of associations between polypharmacy and general health problems, e.g., emergency room visits. This also enables us to consider the definitions as complementary sub-definitions for polypharmacy rather than using them separately and viewing them as alternative definitions.

We will build on this view of the different definitions as representing different levels of information about the medications being used by a given subject to propose a refinement of the definition of polypharmacy.

The next section introduces the methodology we use to detect polypharmacy. Detection algorithm is essentially independent of the specific element or level of information used in the definition of polypharmacy. 


\subsection{Detecting Polypharmacy}

In this work, we used temporal windows and user defined thresholds to determine what would be considered as polypharmacy. Information about prescriptions obtained from the dataset go under three levels of examination by which the three types of polypharmacy can be produced. The result of each polypharmacy definition is then statistically analyzed to provide prevalence analysis. This includes studying the occurrence of single-elements (e.g., single drugs, single classes, or single active ingredients), and the co-occurrence of multiple elements of the same basic type (e.g., two-drugs, two drug classes, two active ingredients, three-drugs, three drug classes, three active ingredients, etc.). Then, we analyze the possible association between polypharmacy combinations of the three levels and ER visits. After that, we estimate the richness for all polypharmacy combinations of multiple elements of the same basic type.

Finally, we observe the thresholds of the result of each polypharmacy definition and study their association with the number of ER visits. We use the result of this analysis to propose a refined definition of polypharmacy using the three levels. Figure 3.1 shows the general workflow for our methodology. 
Figure 3.1: General work flow for our methodology

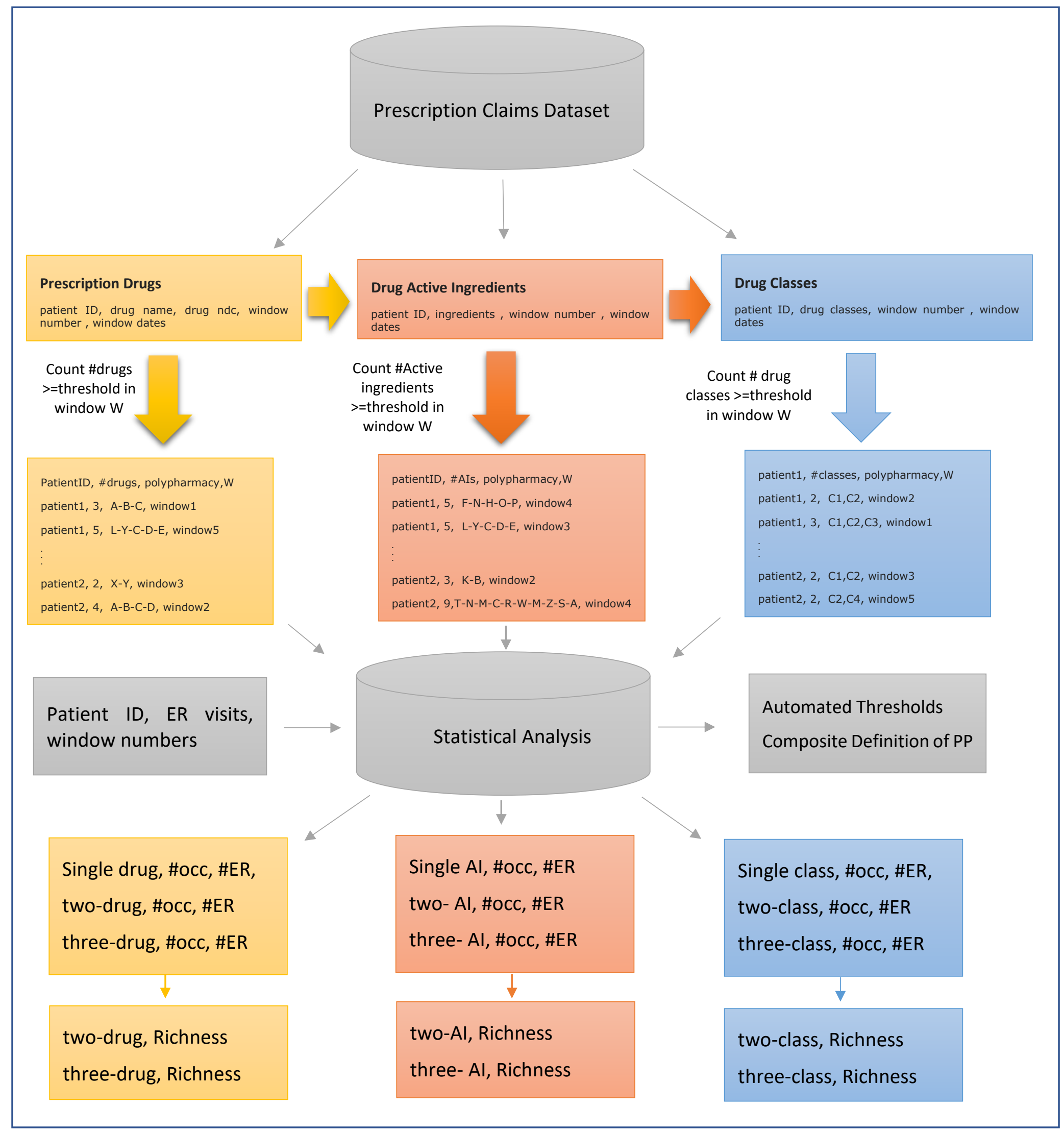


Figure 3.2 shows the algorithm we used to extract polypharmacy for the three definitions.

We run the detection algorithm for all patients in the data set to generate polypharmacy sets for each patient.

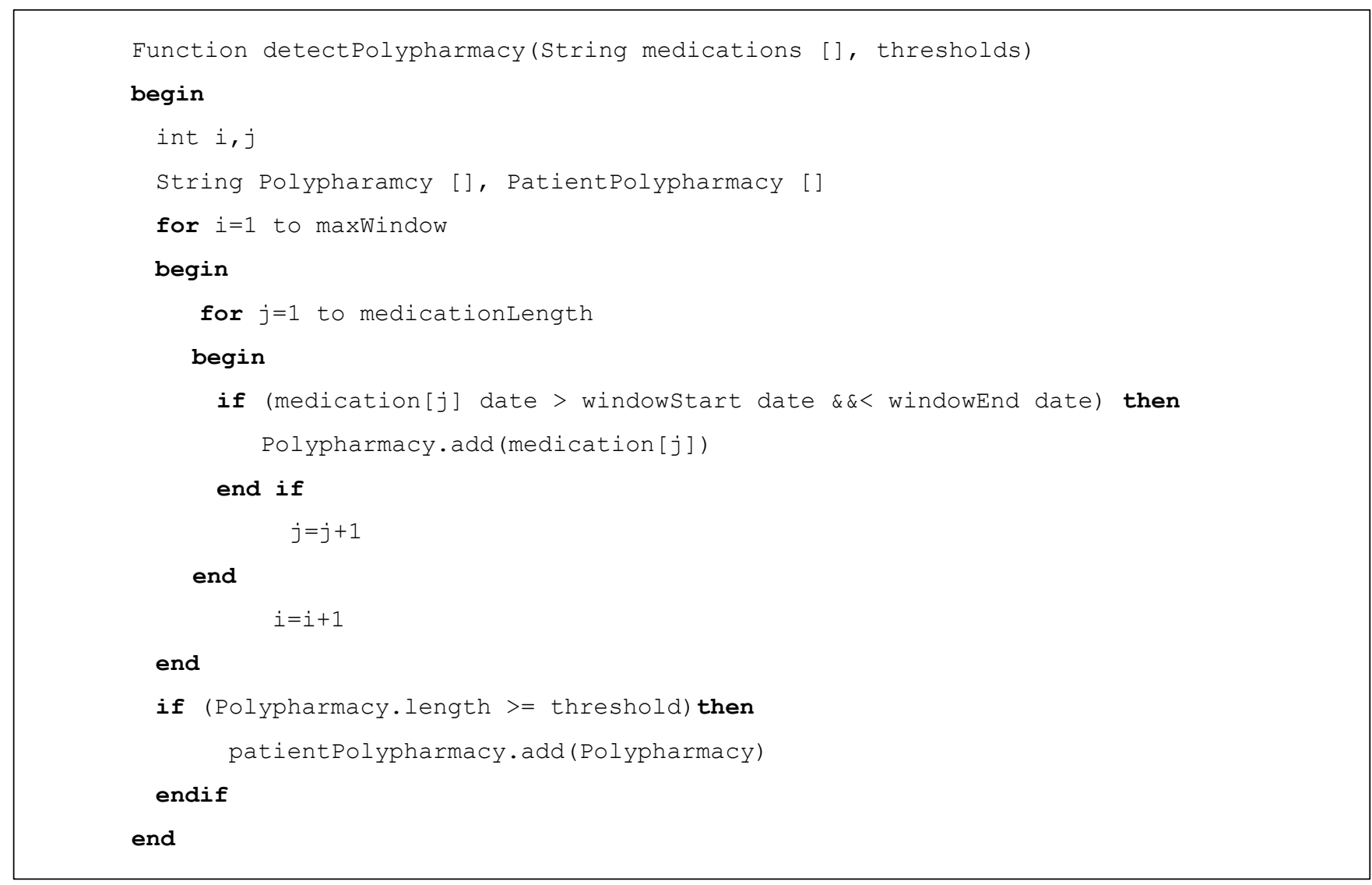

Figure 3.2: Polypharmacy detection algorithm

We used data from the NHANES dataset publicly provided by the U.S. Food and Drug Administration (FDA) to map drugs to their ingredients using National Drug Codes (NDC). Then we map drugs to their drug classes using data provided in our MEPS dataset.

First we apply the algorithm to detect drug polypharmacy. After that, we used a lookup function for retrieving the corresponding ingredients and classes. If the active ingredients were not found by drug NDC, we retry retrieving the ingredients by drug names. If no ingredients were found even after the second attempt, the drug NDC is used in the active ingredients polypharmacy. Finally, the same algorithm in Figure 3.2 is applied to count active ingredients and drug classes within each time window and identify polypharmacy groups based on the given thresholds for each type.

The output from this detection algorithm varies depending on the choice of the thresholds for each: drugs, active ingredients, and drug classes. As discussed earlier in Chapter 2, studies have used various values for these thresholds in an arbitrary manner, without a clear rational 
behind their choices. In fact, determining the problematic thresholds has been considered a challenge in studying polypharmacy[12]. As a consequence, in this study we propose a new method for automating the selection of polypharmacy thresholds by studying different thresholds for each type of polypharmacy and their negative effects on patients. We used the results we obtained to drive a new way for choosing polypharmacy parameters. Next section introduces this contribution.

\subsection{Automated Thresholds for Polypharmacy}

According to a study by Salwe et al [18], "The single most important predictor of inappropriate prescribing and risk of adverse drug events in older patients is the number of prescribed drugs". Polypharmacy problems can be recognized by either identifying threshold on the number of medication elements (drugs, drug classes, active ingredients) or problematic combinations of the elements. In this study, we analyze polypharmacy from both perspectives. This section illustrates our proposed method for defining polypharmacy thresholds. We studied different thresholds for the three definitions of polypharmacy and examined them against a specific measure of risk for ER visits. We varied the thresholds incrementally starting at the simplest value of 1 and then we observe how the ratio

$$
\operatorname{ERPP}(i)=\frac{\# E R(i)}{\# P P(i)}
$$

changes with increasing thresholds, where \#ER(i) = total number of ER visits for cluster $i$, while $\# P P(i)==$ total number of polypharmacy (PP) for cluster $i$. To estimate the change attributed to each threshold, we defined a delta function as follows:

$$
\Delta(k, j)=\frac{\left(\frac{\# E R}{\# P P}\right)_{k}-\left(\frac{\# E R}{\# P P}\right)_{j}}{\left(\frac{\# E R}{\# P P}\right)_{j}}
$$

where $\mathrm{k}$ and $\mathrm{j}$ are two thresholds. To make this comparison more meaningful, we assume intuitively that the least value for the severity of ER problems can be attributed to threshold of value 1 (i.e., single drug, single active ingredient, or single drug class should cause the least ER problems for a given definition of polypharmacy). Based on this assumption, we consider a specific case of the delta function:

$$
\Delta(k, 1)=\frac{\left(\frac{\# E R}{\# P P}\right)_{k}-\left(\frac{\# E R}{\# P P}\right)_{1}}{\left(\frac{\# E R}{\# P P}\right)_{1}}
$$


The percentage threshold can then be chosen by considering the variation of $\Delta(k, 1)$. A specific percentage increase of this measure can be chosen depending on the risk level that is desired to be investigated. We use $50 \%$ increase as an example here.

We calculate the values of the defined ratio $\operatorname{ERPP}(i)$ for each threshold in each year separately and then we averaged the values for all the defined thresholds over the five MEPS years. Overall, we observed that $50 \%$ increase happens at the threshold range of 3-4 for drug polypharmacy. On the other hand, according to the values of this function, it can be observed that $50 \%$ increase in ER measure happens around thresholds 4-5 for active ingredients. With respect to drug classes, this increase happens around the thresholds value of 3-4 values for TC1 and around 5-6 for TC1S1 polypharmacy. Detailed results are presented in Chapter 4. Considering these thresholds for each polypharmacy definition leads to the idea of refining the definition of polypharmacy using these values as sub-thresholds that should be met collectivity. Next section further explains this concept.

\subsection{Refining Definition of Polypharmacy}

The discussion in Section 3.3 leads to the idea of redefining polypharmacy using the thresholds at which a significant increase in ER problems is first observed. As a result, we propose the use of a composite approach to define polypharmacy based on the following criteria:

- Taking $k_{\Delta}$ or more drugs

- Taking $k_{\Delta I}$ or more active ingredients

- Taking $k_{\Delta c}$ or more drug classes by either definition of drug classes.

Where $k_{\Delta}, k_{\Delta I}, k_{\Delta c}$ are thresholds for drugs, active ingredients, and drug classes respectively. Then the definition can be specified by joint consideration of the three criteria, this include:

- Strict polypharmacy definition (Using AND Logic on the three definitions)

- Less strict polypharmacy definition (Using OR Logic on the three definitions)

The result is a polypharmacy definition that satisfies on of the following possibility: (Al here means active ingredients)

O Meeting 1 criteria of the three. i.e., the following definitions:

- drug OR AI OR either type of drug classes

- Drug OR Al

- Drug OR either type of drug classes

- Al OR either type of drug classes

- Meeting 2 criteria of the three (with at most one of the drug classes thresholds). i.e., the following definitions: 
- Drug AND Al

- Drug AND either type of drug classes

- Al AND either type of drug classes

- Meeting all criteria of the three (with at most one of the drug classes thresholds) i.e., the following definition:

- Drug AND AI AND either type of drug classes

We calculate the values of the ratio, $\operatorname{ERPP}(i)=\frac{\# E R(i)}{\# P P(i)}$ for each composite definition and compare the results in Chapter 4.

The choice here could depend on the application at hand. To put this in perspective, a person taking 3 drugs that involve two active ingredients and one drug class might not be exposed to harm as much as a person taking three drugs with five active ingredients and three classes. Such discussion on thresholds and strictness of polypharmacy definition can be further enriched by physicians, pharmacologists, and health care specialists to derive more insightful conclusions.

Chapter 4 exhibits a computational and comparative analysis for polypharmacy extracted from popular data using the three definitions.

\subsection{Computational Analysis of the Three Definitions}

In this work, we perform a computational analysis of the three different approaches to defining polypharmacy. First, we statistically analyze the prevalence of polypharmacy using different medication elements. This includes studying the occurrence of single-elements (e.g., single drugs, single classes, or single active ingredients), and the co-occurrence of multiple elements of the same basic type (e.g., two-drugs, two drug classes, two active ingredients, threedrugs, three drug classes, three active ingredients, etc.). Then, we analyze the possible association between polypharmacy combinations of the three levels and ER visits. After that, we estimate the richness for all polypharmacy combinations of multiple elements of the same basic type

\subsubsection{Prevalence}

Multiple variables are used to analyze the prevalence of each polypharmacy definition. They include: the average number of elements in the polypharmacy, the number of polypharmacy sets, the average number of polypharmacy per patient, the maximum thresholds found for polypharmacy sets, and the counts of occurrence and co-occurrence of multiple elements for polypharmacy. This analysis also summarizes the top polypharmacy combinations

of drugs, active ingredients, and drug classes that were ranked based on the ER visits they were associated with. 


\subsubsection{Association of Polypharmacy with ER}

In this study, we have used three measures to investigate relationships between different polypharmacy definitions and the number of emergency room visits for patients consuming those multiple drugs. We first considered the number of elements in each polypharmacy as a leading predictor for problems connected to ER visits. Thus, we clustered polypharmacy results into groups based on the number of drugs, number of active ingredients, or number of drug classes, respectively. Then we summed up the number of ER visits for every group and normalized it by the number of polypharmacy instances found in the same cluster as illustrated by the following ratio:

$$
\begin{gathered}
\operatorname{ERPP}(i)=\frac{\text { Total of } E R \text { visitis for cluster }(i)}{\text { Total number of PP in cluster }(i)} \\
=\frac{\# E R(i)}{\# P P(i)}
\end{gathered}
$$

where (i) indicates the clusters: 1-element, 2-4 elements, 5-8 elements, 9-12 elements, and all elements $>12$; and PP stands for polypharmacy.

The second measure indicated the percentage that this cluster represents with respect to the total number of ER visits for the whole year. This is defined as the following rate:

$$
\frac{\# E R(i)}{\text { Total } \# E R_{S}}=\frac{\# E R(i)}{\sum_{i} \# E R(i)}
$$

Finally, we calculated the probability that a patient in cluster(i) would visit the ER using the following fraction:

- $\quad$ Cluster ER Probability, $\operatorname{ERP}(\mathrm{i})=\frac{\# E R(i)}{\# \text { patients }(i)}=\frac{\# E R(i)}{\# P T(i)}$

Where \#pt(i)= number of patients in cluster $i$.

$$
\operatorname{PPER} 1(i)=\frac{E R P(i)}{\sum_{j} \operatorname{ERP}(j)}
$$

Assuming conditional independence; we can also measure the probability as follows:

- $\quad \operatorname{ERP2}(\mathrm{i})=\frac{1}{\# P T(i)} \frac{\# E R(i)}{\sum_{h} \# E R(h)}$

$$
\operatorname{PPER} 2(i)=\frac{E R P 2(i)}{\sum_{h} \# E R P 2(h)}
$$

It is important to notice that a patient can belong to multiple groups as patients would have multiple polypharmacy each of which has different number of elements. Independent of the basis 
used for the polypharmacy definition, the results showed a general increase in ER problems with increasing number of drugs/drug classes/or active ingredients. Details on the results on analysis of these three measures can be found in Chapter 4.

\subsubsection{Richness and Over-Representation}

Besides identifying polypharmacy lists and their associations with ER admissions, we analyze the richness for each polypharmacy by which we study how the expected observation of polypharmacy in relation to its actual observation. This is defined by the following ratio:

$$
\text { Richness }=\frac{\text { \#actual observation }}{\text { \#expected observation }}
$$

For cases involving two elements, say $A$ and $B$, this is mathematically equivalent to the following:

$$
\text { Richness }(\mathrm{A}, \mathrm{B})=\frac{P(A \text { and } B)}{P(A) * P(B)}
$$

where $A$ and $B$ are drugs, active ingredients, or drug classes, and $\mathrm{P}$ is probability.

The probability for a single element (e) is calculated as:

$$
P(e)=\frac{\# \operatorname{occ}(e)}{\sum_{j} \# \operatorname{occ}(j)}
$$

Where \#occ $(e)=$ number of occurrences of element $e$.

While the probability for two-element and three-element polypharmacy are calculated as:

$$
P\left(e_{1} \text { and } e_{2}\right)=\frac{\# \operatorname{occ}\left(e_{1} \text { and } e_{2}\right)}{\sum_{i} \sum_{j} \# \operatorname{occ}\left(e_{i} \text { and } e_{j}\right)}
$$

(That is, the relative occurrence of this specific $\left(e_{1}\right.$ and $\left.e_{2}\right)$ pair, over the occurrence of all other two-element pairs that appeared in the dataset.)

$$
P\left(e_{1} \text { and } e_{2} \text { and } e_{3}\right)=\frac{\# \operatorname{occ}\left(e_{1} \text { and } e_{2} \text { and } e_{3}\right)}{\sum_{i} \sum_{j} \sum_{k} \# \text { occ }\left(e_{i} \text { and } e_{j} \text { and } e_{k}\right)}
$$

Thus, for the 2-element and 3-element cases, we have: 


$$
\begin{gathered}
\text { Richness }\left(e_{1}, e_{2}\right)=\frac{P\left(e_{1} \text { and } e_{2}\right)}{P\left(e_{1}\right) * P\left(e_{2}\right)} \\
\operatorname{Richness}\left(e_{1}, e_{2}, e_{3}\right)=\frac{P\left(e_{1} \text { and } e_{2} \text { and } e_{3}\right)}{P\left(e_{1}\right) * P\left(e_{2}\right) * P\left(e_{3}\right)}
\end{gathered}
$$

The significance of studying polypharmacy richness can be seen by comparing the likelihood that two or more combinations would appear together with the likelihood of its single element occurrences. For example, if drug $A$ has high probability to appear as a single drug and drug $B$ also has a high probability to appear as a single drug, it would be expected that the two drugs will occur as two-drug polypharmacy i.e. (the polypharmacy: drug A + drug B). The richness measure however would show whether the actual observation agrees or varies with the expected observation. In particular, higher values for richness reflect higher occurrences of the joint polypharmacy in the dataset than expected while lower values mean that the actual observation were lower than expected. We study richness of occurrence, and richness of occurrence with ER visits, for two-element and three-element polypharmacy. Chapter 4 includes richness values for the top overrepresented polypharmacy. 


\section{Chapter 4: Results}

\subsection{Dataset}

Studying prescription claims provides essential background about prescription practices. In fact, monitoring claims was mandated for medication observation based on a recent Act made on Dec. 2016 [32]. In this work, we used prescriptions claims from the Medical Expenditure Panel Survey (MEPS) for outpatients for years 2011-2015. MEPS is a set of largescale national household surveys for noninstitutionalized civilians (i.e., families and individuals, their medical providers, and employers) in the United States [29]. The data was collected from interviews conducted five times during their panels. The interviews are referred to as five rounds. Round dates reflect interview dates, but based on prescription dates the prescriptions were reallocated to round numbers (1-5) representing intervals of about 3 to 6 months. Thus, the temporal windows used to detect polypharmacy in our work are the predefined rounds in MEPS dataset. We extracted patient IDs, round numbers, round start and end dates, emergency room visits, as well as drug NDCs and drug names. Patient claims have already been sorted based on patients IDs by the collectors. Prescriptions that belong to one patient are consecutive within the file of the calendar year. Further details about the data can be found at the MEPS website [29].

Table 4.1 summarizes information on the MEPS datasets from (2011-2015) including the number of patients and number of prescriptions for each year.

Table 4.1: Summary of MEPS dataset characteristics

\begin{tabular}{|l|c|c|c|c|c|c|}
\hline & $\mathbf{2 0 1 1}$ & $\mathbf{2 0 1 2}$ & $\mathbf{2 0 1 3}$ & $\mathbf{2 0 1 4}$ & $\mathbf{2 0 1 5}$ & Total \\
\hline \#Patients & 19168 & 20431 & 19966 & 18963 & 19604 & 98132 \\
\hline \#Prescriptions & 313748 & 324745 & 327558 & 319468 & 330454 & 1615973 \\
\hline \#ER visits & 240994 & 137318 & 163123 & 174012 & 172146 & 887593 \\
\hline \#Panels & $15-16$ & $16-17$ & $17-18$ & $18-19$ & $19-20$ & $15-20$ \\
\hline
\end{tabular}

It is important here to state the assumptions we made while using this dataset. We assume:

- Patients take all medications they were prescribed and started the medications once they get them.

- Repeated drugs or refills within the same time window are not counted.

- ER visits extracted from MEPS files represents the total ER visits for patients within the MEPS year. In particular, there is no indication that those ER visits happened prior or later than the polypharmacy sets detected at a given round. Such information would provide a better view of polypharmacy-ER associations including polypharmacy that might have resulted from ER visits. However, the dataset did not provide such level of details about the ER visits.

- Count of single elements (i.e., drugs, active ingredients, or drug classes) is the count of elements that occurred individually in a single round for a patient, and count of combinations (i.e., two elements, or three elements) is the count of the occurrence of these combinations in a single round for a patient. 
- The occurrence (\#occ) of single element (i.e., drug, active ingredient, or drug class) means the times that this element appears individually in a single round for a patient while the occurrence with ER (\#ER) for a single element means the sum of ER visits for the patient who was prescribed with this single element. These assumptions also apply for combinations of elements. As a result, the occurrence with ER (\#ER) could be higher than the occurrence (\#occ) in some cases.

Next section provides statistical information and observations about the popularity of polypharmacy defined by drugs, drug active ingredients, and drug classes.

\subsection{Prevalence}

The following three sections shows prevalence analysis with regard to each polypharmacy definition. It is important to point that the detection algorithm threshold was adjusted to include every polypharmacy including single-case polypharmacy (single drugs, Als, and drug classes, we still call it polypharmacy even though they apparently may not necessarily be, as we want to use a uniform the terminology to describe the medication elements taken by patients). Also, the maximum number for each polypharmacy definition is fixed at three since this reflects the maximum number of rounds found in MEPS yearly file.

\subsubsection{Prevalence of polypharmacy using number of drugs}

Table 4.2 describes prevalence of polypharmacy defined by number of drugs for the MEPS dataset. It can be observed that the average number of drugs in the polypharmacy sets found by this definition were approximately around 3.3. Generally, a patient would have about 2 polypharmacy. The maximum number of drugs used concurrently by one patient (i.e., in one round) is 38 as reported in 2013. The proportion of single drugs is around $34 \%$ of all polypharmacy while it was around $19 \%$ for two-drug combinations and nearly $13 \%$ for three-drug combinations.

Table 4.2: Statistics on prevalence of polypharmacy based on number of drugs.

\begin{tabular}{|l|c|c|c|c|c|c|}
\hline & $\mathbf{2 0 1 1}$ & $\mathbf{2 0 1 2}$ & $\mathbf{2 0 1 3}$ & $\mathbf{2 0 1 4}$ & $\mathbf{2 0 1 5}$ & Total \\
\hline Avg of \# drugs in PP & 3.251 & 3.240 & 3.363 & 3.484 & 3.482 & 3.365 \\
\hline $\begin{array}{l}\text { Total number of PP for all } \\
\text { patients }\end{array}$ & 39205 & 41121 & 40130 & 38839 & 40382 & 199677 \\
\hline $\begin{array}{l}\text { Avg number of PP per } \\
\text { patient }\end{array}$ & 2.045 & 2.012 & 2.009 & 2.048 & 2.059 & 2.035 \\
\hline Max \# elements in PP & 33 & 37 & 32 & 38 & 32 & $34.4 * *$ \\
\hline $\begin{array}{l}\text { Max number of } \\
\text { polypharmacy per patient }\end{array}$ & 3 & 3 & 3 & 3 & 3 & 3 \\
\hline Count of single drug & 13,737 & 14,430 & 13,519 & 12,847 & 13,321 & 67854 \\
\hline
\end{tabular}




\begin{tabular}{|l|c|c|c|c|c|c|}
\hline $\begin{array}{l}\text { Count of two-drug } \\
\text { combinations }\end{array}$ & 7798 & 8265 & 7913 & 7613 & 7892 & 39481 \\
\hline $\begin{array}{l}\text { Count of three-drug } \\
\text { combinations }\end{array}$ & 5062 & 5391 & 5286 & 4898 & 5138 & 26861 \\
\hline
\end{tabular}

** Average value is indicated

We extracted drugs that patients take in each round. The resulting sets include combinations of single drugs, two-drugs, and three drugs, and so on. Then we summarized the results of similar drugs for different patients and different years by adding their counts. The result is a table for each possible combination with their counts in each year and collectively in all the 5 years. In our work, we only focus on the first three combinations. Table 4.3, Table 4.4, and Table 4.5 show the top 30 polypharmacy sets of single drugs, two-drugs, and three drugs, respectively, ranked by their occurrence in every year and in total of the five years.

Table 4.3: Top 30 single-drugs in the dataset ranked by their occurrences

\begin{tabular}{|c|c|c|c|c|c|c|c|}
\hline & & \multicolumn{5}{|c|}{ Number of Occurrence } & \multirow[b]{2}{*}{ Total } \\
\hline & Single-drugs & 2011 & 2012 & 2013 & 2014 & 2015 & \\
\hline 1 & AMOXICILLIN & 931 & 958 & 857 & 835 & 890 & 4,471 \\
\hline 2 & AZITHROMYCIN & 732 & 848 & 706 & 694 & 200 & 3,180 \\
\hline 3 & LISINOPRIL & 309 & 392 & 348 & 330 & 358 & 1,737 \\
\hline 4 & LEVOTHYROXIN & 290 & 335 & 326 & 354 & 390 & 1,695 \\
\hline 5 & IBUPROFEN & 271 & 333 & 368 & 332 & 371 & 1,675 \\
\hline 6 & HYDROCO/APAP & 282 & 264 & 236 & 255 & 201 & 1,238 \\
\hline 7 & SIMVASTATIN & 284 & 246 & 180 & 156 & 148 & 1,014 \\
\hline 8 & ALBUTEROL & 156 & 207 & 149 & 142 & 131 & 785 \\
\hline 9 & METFORMIN & 166 & 174 & 146 & 134 & 160 & 780 \\
\hline 10 & OMEPRAZOLE & 101 & 152 & 145 & 140 & 183 & 721 \\
\hline 11 & METHYLPHENID & 42 & 145 & 146 & 178 & 187 & 698 \\
\hline 12 & AMLODIPINE & 114 & 136 & 127 & 114 & 193 & 684 \\
\hline 13 & AMPHETAMINE & 91 & 103 & 121 & 158 & 153 & 626 \\
\hline 14 & APAP/CODEINE & 138 & 139 & 149 & 106 & 82 & 614 \\
\hline 15 & ATORVASTATIN & 1 & 92 & 128 & 141 & 194 & 556 \\
\hline 16 & SERTRALINE & 74 & 118 & 118 & 114 & 115 & 539 \\
\hline 17 & LISINOP/HCTZ & 90 & 115 & 130 & 87 & 117 & 539 \\
\hline 18 & CEPHALEXIN & 109 & 101 & 123 & 115 & 90 & 538 \\
\hline 19 & SYNTHROID & 122 & 120 & 99 & 92 & 104 & 537 \\
\hline 20 & VYVANSE & 114 & 95 & 112 & 109 & 102 & 532 \\
\hline 21 & NAPROXEN & 88 & 101 & 117 & 90 & 97 & 493 \\
\hline 22 & AMOX/K CLAV & & 90 & 100 & 82 & 213 & 485 \\
\hline 23 & FLUTICASONE & 77 & 98 & 104 & 105 & 83 & 467 \\
\hline 24 & HYDROCHLOROT & 78 & 98 & 79 & 102 & 106 & 463 \\
\hline 25 & PROAIR HFA & & 101 & 110 & 111 & 128 & 450 \\
\hline 26 & CITALOPRAM & 111 & 116 & 90 & 59 & 74 & 450 \\
\hline
\end{tabular}




\begin{tabular}{|l|l|c|c|c|c|c|c|}
\hline 27 & ATENOLOL & 124 & 111 & 81 & 73 & 58 & 447 \\
\hline 28 & PREDNISONE & 89 & 92 & 87 & 97 & 71 & 436 \\
\hline 29 & FLUOXETINE & 76 & 81 & 79 & 65 & 71 & 372 \\
\hline 30 & RANITIDINE & 68 & 70 & 60 & 63 & 66 & 327 \\
\hline
\end{tabular}

The most common drugs prescribed within 5 MEPS years from 2011-2015 are AMOXICILLIN and AZITHROMYCIN which are two common antibiotics. HYDROCO/APAP, IBUPROFEN and APAP/CODEINE are known as strong pain killers that require monitoring by clinicians for usage and dosage.

Table 4.4: Top 30 two-drug polypharmacy ranked by their occurrences

\begin{tabular}{|c|c|c|c|c|c|c|c|}
\hline & & \multicolumn{5}{|c|}{ Number of Occurrence } & \multirow[b]{2}{*}{ Total } \\
\hline & Two-drugs & 2011 & 2012 & 2013 & 2014 & 2015 & \\
\hline 1 & AMOXICILLIN--IBUPROFEN & 39 & 52 & 66 & 52 & 85 & 294 \\
\hline 2 & LISINOPRIL--SIMVASTATIN & 52 & 40 & 47 & 49 & 33 & 221 \\
\hline 3 & HYDROCO/APAP--IBUPROFEN & 33 & 38 & 23 & 55 & 27 & 176 \\
\hline 4 & LISINOPRIL--METFORMIN & 28 & 34 & 33 & 31 & 34 & 160 \\
\hline 5 & HYDROCHLOROT--LISINOPRIL & 26 & 35 & 24 & 37 & 37 & 159 \\
\hline 6 & AMOXICILLIN--HYDROCO/APAP & 23 & 31 & 33 & 40 & 30 & 157 \\
\hline 7 & AMLODIPINE--LISINOPRIL & 23 & 18 & 29 & 34 & 39 & 143 \\
\hline 8 & AZITHROMYCIN--IBUPROFEN & 26 & 29 & 26 & 31 & 8 & 120 \\
\hline 9 & APAP/CODEINE--AZITHROMYCIN & 28 & 34 & 29 & 24 & 1 & 116 \\
\hline 10 & METFORMIN--SIMVASTATIN & 21 & 22 & 23 & 15 & 23 & 104 \\
\hline 11 & AMOXICILLIN--AZITHROMYCIN & 21 & 17 & 23 & 22 & 13 & 96 \\
\hline 12 & AMLODIPINE--HYDROCHLOROT & 11 & 22 & 20 & 16 & 27 & 96 \\
\hline 13 & LISINOPRIL--PRAVASTATIN & 21 & 22 & 28 & 16 & 9 & 96 \\
\hline 14 & LEVOTHYROXIN--LISINOPRIL & 16 & 21 & 12 & 21 & 22 & 92 \\
\hline 15 & GLIPIZIDE--METFORMIN & 19 & 15 & 13 & 21 & 21 & 89 \\
\hline 16 & ALBUTEROL--AMOXICILLIN & 15 & 21 & 16 & 17 & 15 & 84 \\
\hline 17 & AZITHROMYCIN--HYDROCO/APAP & 21 & 19 & 17 & 23 & 3 & 83 \\
\hline 18 & CYCLOBENZAPR--IBUPROFEN & 9 & 18 & 21 & 18 & 16 & 82 \\
\hline 19 & CYCLOBENZAPR--HYDROCO/APAP & 16 & 13 & 7 & 23 & 18 & 77 \\
\hline 20 & ATORVASTATIN--LISINOPRIL & & 9 & 18 & 20 & 28 & 75 \\
\hline 21 & LISINOPRIL--OMEPRAZOLE & 7 & 13 & 8 & 17 & 25 & 70 \\
\hline 22 & IBUPROFEN--OXYCOD/APAP & 14 & 8 & 14 & 18 & 13 & 67 \\
\hline 23 & CYCLOBENZAPR--NAPROXEN & 10 & 12 & 19 & 13 & 12 & 66 \\
\hline 24 & AMLODIPINE--LISINOP/HCTZ & 6 & 9 & 13 & 23 & 14 & 65 \\
\hline 25 & AZITHROMYCIN--PREDNISONE & 11 & 13 & 16 & 14 & 9 & 63 \\
\hline 26 & AMOXICILLIN--APAP/CODEINE & 12 & 18 & 17 & 5 & 11 & 63 \\
\hline 27 & ALBUTEROL--AZITHROMYCIN & 24 & 12 & 11 & 8 & 4 & 59 \\
\hline 28 & LEVOTHYROXIN--SIMVASTATIN & 7 & 14 & 16 & 9 & 13 & 59 \\
\hline 29 & HYDROCHLOROT--SIMVASTATIN & 12 & 12 & 11 & 11 & 13 & 59 \\
\hline 30 & LISINOP/HCTZ--SIMVASTATIN & 13 & 15 & 18 & 10 & 2 & 58 \\
\hline
\end{tabular}


Considering the single-occurrences in Table 4.3 and the co-occurrences of pairs in Table 4.4, we can observe that among the most common drugs are AMOXICILLIN which occurred 4,471 times and IBUPROFEN which occurred 1,675 times. However, the pair (AMOXICILLIN-IBUPROFEN) occurred much less with occurrence total of 294 . This indicates that the cooccurrence of two drugs in a polypharmacy does not necessarily align with the occurrence of the individual drugs involved in that polypharmacy. However, in this particular case, the pair (AMOXICILLIN--IBUPROFEN) was the highest occurring two-drug polypharmacy.

Table 4.5: Top 30 three-drug polypharmacy ranked by their occurrences

\begin{tabular}{|c|c|c|c|c|c|c|c|}
\hline & \multirow[b]{2}{*}{ Three-drugs } & \multicolumn{5}{|c|}{ Number of Occurrence } & \multirow[b]{2}{*}{ Total } \\
\hline & & 2011 & 2012 & 2013 & 2014 & 2015 & \\
\hline 1 & LISINOPRIL--METFORMIN--SIMVASTATIN & 12 & 10 & 10 & 12 & 12 & 56 \\
\hline 2 & ATORVASTATIN--LISINOPRIL--METFORMIN & & 6 & 8 & 12 & 11 & 37 \\
\hline 3 & LISINOPRIL--METFORMIN--PRAVASTATIN & 5 & 7 & 4 & 3 & 16 & 35 \\
\hline 4 & GLIPIZIDE--LISINOPRIL--METFORMIN & 6 & 13 & 5 & 4 & 5 & 33 \\
\hline 5 & HYDROCHLOROT--LISINOPRIL--SIMVASTATIN & 4 & 9 & 6 & 8 & 3 & 30 \\
\hline 6 & CYCLOBENZAPR--HYDROCO/APAP--IBUPROFEN & 6 & 7 & 6 & 4 & 6 & 29 \\
\hline 7 & LISINOPRIL--LOVASTATIN--METFORMIN & 3 & 6 & 1 & 8 & 6 & 24 \\
\hline 8 & AMOXICILLIN--HYDROCO/APAP--IBUPROFEN & 4 & 2 & 6 & 4 & 4 & 20 \\
\hline 9 & HYDROCHLOROT--LISINOPRIL--METFORMIN & 4 & 3 & 1 & 7 & 5 & 20 \\
\hline 10 & AMLODIPINE--HYDROCHLOROT--LISINOPRIL & 3 & 6 & 8 & 2 & 1 & 20 \\
\hline 11 & GLYBURIDE--LISINOPRIL--METFORMIN & 3 & 7 & 6 & & 1 & 17 \\
\hline 12 & LISINOPRIL--METOPROLOL--SIMVASTATIN & 6 & 4 & 3 & 4 & & 17 \\
\hline 13 & AMLODIPINE--LISINOPRIL--SIMVASTATIN & 4 & 7 & 1 & 1 & 2 & 15 \\
\hline 14 & HYDROCHLOROT--LISINOPRIL--PRAVASTATIN & 2 & 6 & 4 & 2 & & 14 \\
\hline 15 & LEVOTHYROXIN--SIMVASTATIN--TRIAMT/HCTZ & 2 & 4 & 2 & 3 & 2 & 13 \\
\hline 16 & LEVOTHYROXIN--LISINOPRIL--SIMVASTATIN & 2 & 1 & & 5 & 5 & 13 \\
\hline 17 & AMLODIPINE--LEVOTHYROXIN--SIMVASTATIN & 2 & 5 & & 3 & 3 & 13 \\
\hline 18 & GLIMEPIRIDE--LISINOPRIL--METFORMIN & 2 & 1 & 3 & 3 & 4 & 13 \\
\hline 19 & ATENOLOL--HYDROCHLOROT--SIMVASTATIN & 3 & 5 & 5 & & & 13 \\
\hline 20 & LEVOTHYROXIN--LISINOPRIL--PRAVASTATIN & & 4 & 2 & 4 & 2 & 12 \\
\hline 21 & AMLODIPINE--METOPROL TAR--SIMVASTATIN & & 4 & 4 & 3 & 1 & 12 \\
\hline 22 & LISINOPRIL--METOPROL TAR--SIMVASTATIN & & 5 & & 4 & 3 & 12 \\
\hline 23 & CARVEDILOL--LISINOPRIL--PRAVASTATIN & 4 & 5 & 2 & 1 & & 12 \\
\hline 24 & ATORVASTATIN--LISINOPRIL--METOPROL TAR & & 1 & 3 & 3 & 5 & 12 \\
\hline 25 & ALBUTEROL--AZITHROMYCIN--PREDNISOLONE & 3 & 4 & 1 & 2 & 1 & 11 \\
\hline 26 & ALBUTEROL--AZITHROMYCIN--PREDNISONE & 2 & 5 & 2 & 2 & & 11 \\
\hline 27 & GLIPIZIDE--METFORMIN--PRAVASTATIN & 4 & 3 & 1 & 2 & 1 & 11 \\
\hline 28 & LISINOP/HCTZ--METFORMIN--SIMVASTATIN & 3 & & 1 & 4 & 3 & 11 \\
\hline 29 & LEVOTHYROXIN--LISINOPRIL--METFORMIN & 1 & & 5 & & 4 & 10 \\
\hline 30 & CYCLOBENZAPR--HYDROCO/APAP--NAPROXEN & & 4 & 4 & 1 & 1 & 10 \\
\hline
\end{tabular}


From Table 4.3, Table 4.4, and Table 4.5 we can see that the occurrence values decrease with the increase in number of drugs (i.e, single-drugs have highest occurrences value followed by two-drugs, followed by three-drugs, and this persists even for combinations containing more than three drugs).

\subsubsection{Prevalence of polypharmacy using number of active ingredients}

Table 4.6 describes the prevalence of polypharmacy using the number of drug active ingredients which was obtained from MEPS five calendar years (2011-2105). As shown in the table, the average number of active ingredients in the polypharmacy sets found by this definition were approximately around 3.7. On average, a patient would have 2.035 cases of polypharmacy. The maximum number of active ingredients used concurrently by one patient (i.e., in one round) is 41 as reported in 2014. The proportion of single active ingredients is about $29 \%$ of all polypharmacy while it is nearly $18 \%$ for two-active ingredient combinations and around $13 \%$ for three-active ingredient combinations.

Table 4.6: Statistics on Prevalence of Polypharmacy using number of active ingredients

\begin{tabular}{|l|c|c|c|c|c|c|}
\hline & $\mathbf{2 0 1 1}$ & $\mathbf{2 0 1 2}$ & $\mathbf{2 0 1 3}$ & $\mathbf{2 0 1 4}$ & $\mathbf{2 0 1 5}$ & Total \\
\hline Avg of \# Als in PP & 3.626 & 3.604 & 3.712 & 3.859 & 3.845 & 3.730 \\
\hline $\begin{array}{l}\text { Total number of PP for all } \\
\text { patients }\end{array}$ & 39205 & 41120 & 40130 & 38839 & 40382 & 199676 \\
\hline $\begin{array}{l}\text { Avg number of PP per } \\
\text { patient }\end{array}$ & 2.045 & 2.012 & 2.009 & 2.048 & 2.059 & 2.035 \\
\hline Max \# elements in PP & 35 & 37 & 32 & 41 & 38 & $36.6 * *$ \\
\hline $\begin{array}{l}\text { Max number of } \\
\text { polypharmacy per patient }\end{array}$ & 3 & 3 & 3 & 3 & 3 & 3 \\
\hline \begin{tabular}{l} 
Count of single Al \\
\hline $\begin{array}{l}\text { Count of two-Al } \\
\text { combinations }\end{array}$
\end{tabular} & 71,964 & 12,631 & 11,798 & 11,258 & 11,342 & 58,993 \\
\hline $\begin{array}{l}\text { Count of three-Al } \\
\text { combinations }\end{array}$ & 5235 & 5679 & 5495 & 5105 & 5398 & 26912 \\
\hline
\end{tabular}

** Average value is indicated

Tables $4.7,4.8$, and 4.9 , respectively, show the top 30 polypharmacy sets for single active ingredients, two- active ingredients, and three active ingredients ranked by occurrence. We can observe that the two-active ingredients (namely, SULFAMETHOXAZOLE and TRIMETHOPRIM) in the top rank for case of two-active ingredients did not even appear in the top 30 ranks for case of single active ingredient prevalence. Similarly, the active ingredient SALMETEROL XINAFOATE which appeared in the top 3-active ingredients did not appear in the top 30 ranks for single active ingredients. 
Table 4.7: Top 30 single-ingredients polypharmacy ranked by their occurrences.

\begin{tabular}{|c|c|c|c|c|c|c|c|}
\hline & \multirow[b]{2}{*}{ Single-ingredients } & \multicolumn{5}{|c|}{ Number of Occurrence } & \multirow[b]{2}{*}{ Total } \\
\hline & & 2011 & 2012 & 2013 & 2014 & 2015 & \\
\hline 1 & AMOXICILLIN & 945 & 962 & 863 & 836 & 899 & 4,505 \\
\hline 2 & AZITHROMYCIN DIHYDRATE & 744 & 869 & 717 & 699 & 204 & 3,233 \\
\hline 3 & LEVOTHYROXINE SODIUM & 457 & 504 & 457 & 459 & 516 & 2,393 \\
\hline 4 & ALBUTEROL SULFATE & 307 & 402 & 416 & 355 & 395 & 1,875 \\
\hline 5 & LISINOPRIL & 309 & 392 & 348 & 330 & 358 & 1,737 \\
\hline 6 & IBUPROFEN & 283 & 337 & 371 & 333 & 372 & 1,696 \\
\hline 7 & SIMVASTATIN & 292 & 246 & 180 & 156 & 148 & 1,022 \\
\hline 8 & METHYLPHENIDATE HYDROCHLORIDE & 158 & 202 & 173 & 190 & 207 & 930 \\
\hline 9 & METFORMIN HYDROCHLORIDE & 168 & 178 & 151 & 138 & 167 & 802 \\
\hline 10 & OMEPRAZOLE & 103 & 153 & 145 & 140 & 185 & 726 \\
\hline 11 & AMLODIPINE BESYLATE & 129 & 140 & 131 & 121 & 201 & 722 \\
\hline 12 & FLUTICASONE PROPIONATE & 103 & 144 & 150 & 126 & 104 & 627 \\
\hline 13 & SERTRALINE HYDROCHLORIDE & 99 & 129 & 125 & 124 & 126 & 603 \\
\hline 14 & CEPHALEXIN & 115 & 107 & 126 & 115 & 90 & 553 \\
\hline 15 & LISDEXAMFETAMINE DIMESYLATE & 116 & 95 & 112 & 109 & 102 & 534 \\
\hline 16 & HYDROCHLOROTHIAZIDE & 106 & 109 & 90 & 109 & 113 & 527 \\
\hline 17 & NAPROXEN & 89 & 101 & 120 & 91 & 98 & 499 \\
\hline 18 & CITALOPRAM HYDROBROMIDE & 112 & 117 & 91 & 60 & 79 & 459 \\
\hline 19 & MONTELUKAST SODIUM & 97 & 100 & 91 & 85 & 79 & 452 \\
\hline 20 & ATENOLOL & 124 & 111 & 81 & 73 & 58 & 447 \\
\hline 21 & PREDNISONE & 89 & 92 & 88 & 98 & 71 & 438 \\
\hline 22 & ATORVASTATIN CALCIUM TRIHYDRATE & 117 & 103 & 35 & 92 & 79 & 426 \\
\hline 23 & METOPROLOL TARTRATE & 103 & 97 & 69 & 75 & 79 & 423 \\
\hline 24 & FLUOXETINE HYDROCHLORIDE & 81 & 90 & 89 & 71 & 73 & 404 \\
\hline 25 & LORATADINE & 78 & 76 & 56 & 66 & 74 & 350 \\
\hline 26 & RANITIDINE HYDROCHLORIDE & 73 & 74 & 60 & 69 & 67 & 343 \\
\hline 27 & CETIRIZINE HYDROCHLORIDE & 58 & 54 & 66 & 85 & 72 & 335 \\
\hline 28 & PENICILLIN V POTASSIUM & 63 & 52 & 71 & 79 & 63 & 328 \\
\hline 29 & OSELTAMIVIR PHOSPHATE & 41 & 46 & 86 & 79 & 62 & 314 \\
\hline 30 & $555904358 * *$ & 138 & 33 & 33 & 96 & 14 & 314 \\
\hline
\end{tabular}

**Drug NDC is used when no active ingredients cannot be found. 
Table 4.8: Top 30 two-active ingredient polypharmacy ranked by their occurrences

\begin{tabular}{|c|c|c|c|c|c|c|c|}
\hline & \multirow[b]{2}{*}{ Two-ingredients } & \multicolumn{5}{|c|}{ Number of Occurrence } & \multirow[b]{2}{*}{ Total } \\
\hline & & 2011 & 2012 & 2013 & 2014 & 2015 & \\
\hline 1 & ACETAMINOPHEN--HYDROCODONE BITARTRATE & 216 & 192 & 199 & 285 & 227 & 1119 \\
\hline 2 & HYDROCHLOROTHIAZIDE--LISINOPRIL & 120 & 157 & 172 & 127 & 159 & 735 \\
\hline 3 & AMOXICILLIN--CLAVULANATE POTASSIUM & 118 & 113 & 126 & 103 & 218 & 678 \\
\hline 4 & ACETAMINOPHEN--CODEINE PHOSPHATE & 147 & 146 & 146 & 107 & 86 & 632 \\
\hline 5 & SULFAMETHOXAZOLE--TRIMETHOPRIM & 71 & 98 & 71 & 76 & 192 & 508 \\
\hline 6 & ACETAMINOPHEN--OXYCODONE HYDROCHLORIDE & 107 & 86 & 74 & 67 & 106 & 440 \\
\hline 7 & ETHINYL ESTRADIOL--NORETHINDRONE ACETATE & 59 & 67 & 55 & 61 & 69 & 311 \\
\hline 8 & AMOXICILLIN--IBUPROFEN & 45 & 52 & 67 & 52 & 85 & 301 \\
\hline 9 & ETONOGESTREL--ETHINYL ESTRADIOL & 62 & 52 & 53 & 38 & 43 & 248 \\
\hline 10 & HYDROCHLOROTHIAZIDE--LOSARTAN POTASSIUM & 26 & 52 & 41 & 47 & 69 & 235 \\
\hline 11 & LISINOPRIL--SIMVASTATIN & 53 & 40 & 47 & 49 & 33 & 222 \\
\hline 12 & $\begin{array}{l}\text { FLUTICASONE PROPIONATE--SALMETEROL } \\
\text { XINAFOATE }\end{array}$ & 61 & 45 & 34 & 25 & 54 & 219 \\
\hline 13 & ALBUTEROL SULFATE--FLUTICASONE PROPIONATE & 37 & 49 & 34 & 36 & 34 & 190 \\
\hline 14 & $\begin{array}{l}\text { AMLODIPINE BESYLATE--BENAZEPRIL } \\
\text { HYDROCHLORIDE }\end{array}$ & 44 & 42 & 26 & 35 & 34 & 181 \\
\hline 15 & BISOPROLOL FUMARATE--HYDROCHLOROTHIAZIDE & 77 & 67 & 10 & 7 & 10 & 171 \\
\hline 16 & LISINOPRIL--METFORMIN HYDROCHLORIDE & 31 & 33 & 33 & 31 & 35 & 163 \\
\hline 17 & AMLODIPINE BESYLATE--LISINOPRIL & 23 & 19 & 29 & 36 & 40 & 147 \\
\hline 18 & ATENOLOL--CHLORTHALIDONE & 44 & 40 & 30 & 23 & 8 & 145 \\
\hline 19 & ALBUTEROL SULFATE--MONTELUKAST SODIUM & 37 & 26 & 31 & 22 & 22 & 138 \\
\hline 20 & $\begin{array}{l}\text { HYDROCHLOROTHIAZIDE--PROPRANOLOL } \\
\text { HYDROCHLORIDE }\end{array}$ & 1 & 2 & 68 & 61 & & 132 \\
\hline 21 & HYDROCHLOROTHIAZIDE--TRIAMTERENE & 24 & 27 & 14 & 28 & 33 & 126 \\
\hline 22 & AZITHROMYCIN DIHYDRATE--IBUPROFEN & 26 & 30 & 27 & 31 & 8 & 122 \\
\hline 23 & AMLODIPINE BESYLATE--HYDROCHLOROTHIAZIDE & 16 & 23 & 24 & 20 & 30 & 113 \\
\hline 24 & METFORMIN HYDROCHLORIDE--SIMVASTATIN & 21 & 24 & 26 & 15 & 23 & 109 \\
\hline 25 & $\begin{array}{l}\text { ALBUTEROL SULFATE--BECLOMETHASONE } \\
\text { DIPROPIONATE }\end{array}$ & 18 & 30 & 32 & 11 & 13 & 104 \\
\hline 26 & HYDROCHLOROTHIAZIDE--VALSARTAN & 30 & 32 & 12 & 11 & 19 & 104 \\
\hline 27 & AMOXICILLIN--ALBUTEROL SULFATE & 20 & 23 & 17 & 20 & 23 & 103 \\
\hline 28 & GLIPIZIDE--METFORMIN HYDROCHLORIDE & 25 & 16 & 15 & 23 & 22 & 101 \\
\hline 29 & ALBUTEROL SULFATE--BUDESONIDE & 22 & 21 & 18 & 22 & 17 & 100 \\
\hline 30 & CYCLOBENZAPRINE HYDROCHLORIDE--IBUPROFEN & 16 & 19 & 26 & 19 & 18 & 98 \\
\hline
\end{tabular}


Table 4.9: Top 30 three-active ingredient polypharmacy ranked by their occurrences

\begin{tabular}{|c|c|c|c|c|c|c|c|}
\hline & \multirow[b]{2}{*}{ Three-ingredients } & \multicolumn{5}{|c|}{ Number of Occurrence } & \multirow[b]{2}{*}{ Total } \\
\hline & & 2011 & 2012 & 2013 & 2014 & 2015 & \\
\hline 1 & $\begin{array}{l}\text { ALBUTEROL SULFATE--FLUTICASONE PROPIONATE-- } \\
\text { SALMETEROL XINAFOATE }\end{array}$ & 40 & 38 & 38 & 25 & 24 & 165 \\
\hline 2 & $\begin{array}{l}\text { ACETAMINOPHEN--HYDROCODONE BITARTRATE-- } \\
\text { IBUPROFEN }\end{array}$ & 24 & 30 & 28 & 53 & 28 & 163 \\
\hline 3 & $\begin{array}{l}\text { BROMPHENIRAMINE MALEATE--DEXTROMETHORPHAN } \\
\text { HYDROBROMIDE--PSEUDOEPHEDRINE HYDROCHLORIDE }\end{array}$ & 13 & 29 & 47 & 27 & 39 & 155 \\
\hline 4 & $\begin{array}{l}\text { HYDROCORTISONE--NEOMYCIN SULFATE--POLYMYXIN B } \\
\text { SULFATE }\end{array}$ & 23 & 24 & 34 & 25 & 17 & 123 \\
\hline 5 & $\begin{array}{l}\text { AMLODIPINE BESYLATE--HYDROCHLOROTHIAZIDE-- } \\
\text { LISINOPRIL }\end{array}$ & 11 & 17 & 25 & 31 & 17 & 101 \\
\hline 6 & HYDROCHLOROTHIAZIDE--LISINOPRIL--SIMVASTATIN & 20 & 25 & 25 & 18 & 5 & 93 \\
\hline 7 & $\begin{array}{l}\text { AMOXICILLIN--ACETAMINOPHEN--HYDROCODONE } \\
\text { BITARTRATE }\end{array}$ & 12 & 10 & 14 & 37 & 18 & 91 \\
\hline 8 & $\begin{array}{l}\text { ACETAMINOPHEN--IBUPROFEN--OXYCODONE } \\
\text { HYDROCHLORIDE }\end{array}$ & 15 & 11 & 19 & 20 & 16 & 81 \\
\hline 9 & $\begin{array}{l}\text { ACETAMINOPHEN--CYCLOBENZAPRINE } \\
\text { HYDROCHLORIDE--HYDROCODONE BITARTRATE }\end{array}$ & 16 & 11 & 9 & 22 & 18 & 76 \\
\hline 10 & $\begin{array}{l}\text { AZITHROMYCIN DIHYDRATE--ACETAMINOPHEN-- } \\
\text { CODEINE PHOSPHATE }\end{array}$ & 19 & 18 & 20 & 14 & 1 & 72 \\
\hline 11 & $\begin{array}{l}\text { AMLODIPINE BESYLATE--HYDROCHLOROTHIAZIDE-- } \\
\text { LOSARTAN POTASSIUM }\end{array}$ & 9 & 10 & 19 & 12 & 20 & 70 \\
\hline 12 & ACETAMINOPHEN--BUTALBITAL--CAFFEINE & 10 & 16 & 11 & 16 & 10 & 63 \\
\hline 13 & $\begin{array}{l}\text { LISINOPRIL--METFORMIN HYDROCHLORIDE-- } \\
\text { SIMVASTATIN }\end{array}$ & 12 & 10 & 14 & 13 & 13 & 62 \\
\hline 14 & $\begin{array}{l}\text { ACETAMINOPHEN--HYDROCODONE BITARTRATE-- } \\
\text { OXYCODONE HYDROCHLORIDE }\end{array}$ & 8 & 11 & 8 & 15 & 13 & 55 \\
\hline 15 & $\begin{array}{l}\text { ACETAMINOPHEN--HYDROCODONE BITARTRATE-- } \\
\text { PENICILLIN V POTASSIUM }\end{array}$ & 11 & 4 & 12 & 20 & 8 & 55 \\
\hline 16 & ACETAMINOPHEN--CODEINE PHOSPHATE--IBUPROFEN & 9 & 9 & 9 & 11 & 12 & 50 \\
\hline 17 & $\begin{array}{l}\text { HYDROCHLOROTHIAZIDE--LISINOPRIL--METFORMIN } \\
\text { HYDROCHLORIDE }\end{array}$ & 11 & 11 & 4 & 12 & 12 & 50 \\
\hline 18 & $\begin{array}{l}\text { ACETAMINOPHEN--AMOXICILLIN--HYDROCODONE } \\
\text { BITARTRATE }\end{array}$ & 8 & 8 & 11 & 8 & 14 & 49 \\
\hline 19 & $\begin{array}{l}\text { ACETAMINOPHEN--AZITHROMYCIN DIHYDRATE-- } \\
\text { CODEINE PHOSPHATE }\end{array}$ & 10 & 15 & 10 & 14 & & 49 \\
\hline 20 & AMOXICILLIN--ACETAMINOPHEN--CODEINE PHOSPHATE & 7 & 13 & 14 & 4 & 11 & 49 \\
\hline 21 & $\begin{array}{l}\text { ACETAMINOPHEN--CEPHALEXIN--HYDROCODONE } \\
\text { BITARTRATE }\end{array}$ & 15 & 6 & 8 & 16 & 3 & 48 \\
\hline 22 & $\begin{array}{l}\text { AZITHROMYCIN DIHYDRATE--ACETAMINOPHEN-- } \\
\text { HYDROCODONE BITARTRATE }\end{array}$ & 10 & 10 & 8 & 18 & 1 & 47 \\
\hline 23 & $\begin{array}{l}\text { ALBUTEROL SULFATE--FLUTICASONE PROPIONATE-- } \\
\text { MONTELUKAST SODIUM }\end{array}$ & 13 & 6 & 15 & 9 & 3 & 46 \\
\hline
\end{tabular}




\begin{tabular}{|c|l|c|c|c|c|c|c|}
\hline 24 & $\begin{array}{l}\text { HYDROCHLOROTHIAZIDE--LISINOPRIL--PRAVASTATIN } \\
\text { SODIUM }\end{array}$ & 10 & 12 & 11 & 5 & 4 & 42 \\
\hline 25 & $\begin{array}{l}\text { AZITHROMYCIN DIHYDRATE--CODEINE PHOSPHATE-- } \\
\text { GUAIFENESIN }\end{array}$ & 7 & 19 & 5 & 3 & 7 & 41 \\
\hline 26 & GLIPIZIDE--LISINOPRIL--METFORMIN HYDROCHLORIDE & 10 & 14 & 8 & 4 & 5 & 41 \\
\hline 27 & $\begin{array}{l}\text { BROMPHENIRAMINE MALEATE--DEXTROMETHORPHAN } \\
\text { HYDROBROMIDE--PHENYLEPHRINE HYDROCHLORIDE }\end{array}$ & 9 & 5 & 20 & 4 & 2 & 40 \\
\hline 28 & LISINOPRIL--METOPROLOL TARTRATE--SIMVASTATIN & 15 & 9 & 3 & 8 & 4 & 39 \\
\hline 29 & $\begin{array}{l}\text { HYDROCHLOROTHIAZIDE--LISINOPRIL--METOPROLOL } \\
\text { TARTRATE }\end{array}$ & 3 & 5 & 6 & 10 & 13 & 37 \\
\hline 30 & $\begin{array}{l}\text { LISINOPRIL-- METFORMIN } \\
\text { HYDROCHLORIDE\#PRAVASTATIN SODIUM }\end{array}$ & 5 & 7 & 4 & 3 & 16 & 35 \\
\hline
\end{tabular}

It can be seen from the three Tables $4.7,4.8$, and 4.9 that some active ingredients names are similar to the original drugs. This is because some drugs have only one active ingredient and so are named based on the substance. Many drugs can share the exact sets of active ingredients or subsets of them. As a consequence, the occurrences of single or multiple active ingredients may be originated from different drugs. Table 4.10 shows some instances of active ingredients matched with the drugs they compose.

Table 4.10: List of ingredients/substances matched with their parent drugs.

\begin{tabular}{|l|l|}
\hline Substance name & Drugs Name \\
\hline ZOLEDRONIC ACID & $\begin{array}{l}\text { Reclast } \\
\text { Zometa }\end{array}$ \\
\hline ZOLPIDEM TARTRATE & $\begin{array}{l}\text { Ambien CR } \\
\text { Edluar } \\
\text { Intermezzo } \\
\text { ZolpiMIST }\end{array}$ \\
\hline $\begin{array}{l}\text { BRIMONIDINE } \\
\text { TARTRATE }\end{array}$ & $\begin{array}{l}\text { Brimonidine Tartrate Ophthalmic Solution, 0.2\% } \\
\text { LUMIFY Redness Reliever Eye Drops } \\
\text { Mirvaso } \\
\text { ALPHAGAN P }\end{array}$ \\
\hline $\begin{array}{l}\text { ADAPALENE--BENZOYL } \\
\text { PEROXIDE }\end{array}$ & $\begin{array}{l}\text { Epiduo forte } \\
\text { EPIDUO } \\
\text { ADAPALENE and BENZOYL PEROXIDE } \\
\text { Adapalene and Benzoyl Peroxide } \\
\text { adapalene and benzoyl peroxide }\end{array}$ \\
\hline
\end{tabular}




\begin{tabular}{|c|c|}
\hline $\begin{array}{l}\text { BROMPHENIRAMINE } \\
\text { MALEATE -- } \\
\text { PSEUDOEPHEDRINE } \\
\text { HYDROCHLORIDE }\end{array}$ & $\begin{array}{l}\text { RYNEX PSE } \\
\text { RYNEX PE } \\
\text { Q-Tapp } \\
\text { Lodrane D } \\
\text { Brotapp } \\
\text { Walgreens Fast Acting Heartburn Relief Extra Strength }\end{array}$ \\
\hline $\begin{array}{l}\text { ALUMINUM } \\
\text { HYDROXIDE } \\
-- \\
\text { MAGNESIUM } \\
\text { CARBONATE }\end{array}$ & $\begin{array}{l}\text { SohMed Acid Reducer } \\
\text { RIGINIC ANTACID LIQUID } \\
\text { GOOD NEIGHBOR PHARMACY Antacid Extra Strength } \\
\text { GAVISCON } \\
\text { Gaviscon } \\
\text { GAVIS-CARE ANTACID } \\
\text { Foamcoat Antacid } \\
\text { FAST ACTING Heartburn Relief } \\
\text { EXTRA STRENGTH HEARTBURN RELIEF ANTACID } \\
\text { EXTRA STRENGTH HEARTBURN RELIEF } \\
\text { equate Extra Strength Antacid } \\
\text { CVS Health Extra Strength Heartburn Relief Antacid } \\
\text { Aluminum hydroxide and Magnesium carbonate } \\
\text { ACID GONE ANTACID } \\
\text { Acid Gone Antacid }\end{array}$ \\
\hline $\begin{array}{l}\text { PANCRELIPASE LIPASE } \\
\text {--PANCRELIPASE } \\
\text { PROTEASE } \\
\text {--PANCRELIPASE } \\
\text { AMYLASE }\end{array}$ & $\begin{array}{l}\text { Viokace } \\
\text { ZENPEP } \\
\text { ULTRESA } \\
\text { PANCRELIPASE } \\
\text { Creon }\end{array}$ \\
\hline $\begin{array}{l}\text { CODEINE PHOSPHATE } \\
-- \\
\text { GUAIFENESIN } \\
-- \\
\text { PSEUDOEPHEDRINE } \\
\text { HYDROCHLORIDE }\end{array}$ & $\begin{array}{l}\text { Coditussin DAC } \\
\text { LORTUSS } \\
\text { Tricode } \\
\text { Tusnel } \\
\text { Virtussin DAC }\end{array}$ \\
\hline
\end{tabular}




\begin{tabular}{|l|l|}
\hline BROMPHENIRAMINE & BIO DtUSS \\
MALEATE & BROMPHENIRAMINE MALEATE, PSEUDOEPHEDRINE HYDROCHLORIDE, AND \\
-- & DEXTROMETHORPHAN HYDROBROMIDE \\
$\begin{array}{l}\text { DEXTROMETHORPHAN } \\
\text { HYDROBROMIDE }\end{array}$ & \\
-- & Q Tapp Cold and Cough \\
$\begin{array}{l}\text { PSEUDOEPHEDRINE } \\
\text { HYDROCHLORIDE }\end{array}$ & \\
\hline
\end{tabular}

From Table 4.10 we can see that many drugs that share the same active ingredient(s) have the same name but with some minor changes e.g., different upper vs. lower case, OR shorter vs. longer names. This has been handled by our algorithm by converting all drug names to lower case before performing the lookup function. If the drug at hand was not found using the lookup function and its name, we run the lookup function for the second time using drug NDC.

Using active ingredients approach reveals information about the substances that occurred frequently regardless of their parent drugs. For example, LORATADINE is an active substance for allergy medications. According to our statistics, LORATADINE was ranked 25 based on the occurrence of active ingredients and 40 based on the total of ER visits even though the allergy relief drugs are not ranked high considering ER visits. Also, the substance: HYDROCHLOROTHIAZIDE (ranked 22 by ER) is an active ingredient found in a lot of drugs, which may make observing its patterns prioritized for health care people. Some examples of pairs and triples that include HYDROCHLOROTHIAZIDE are:

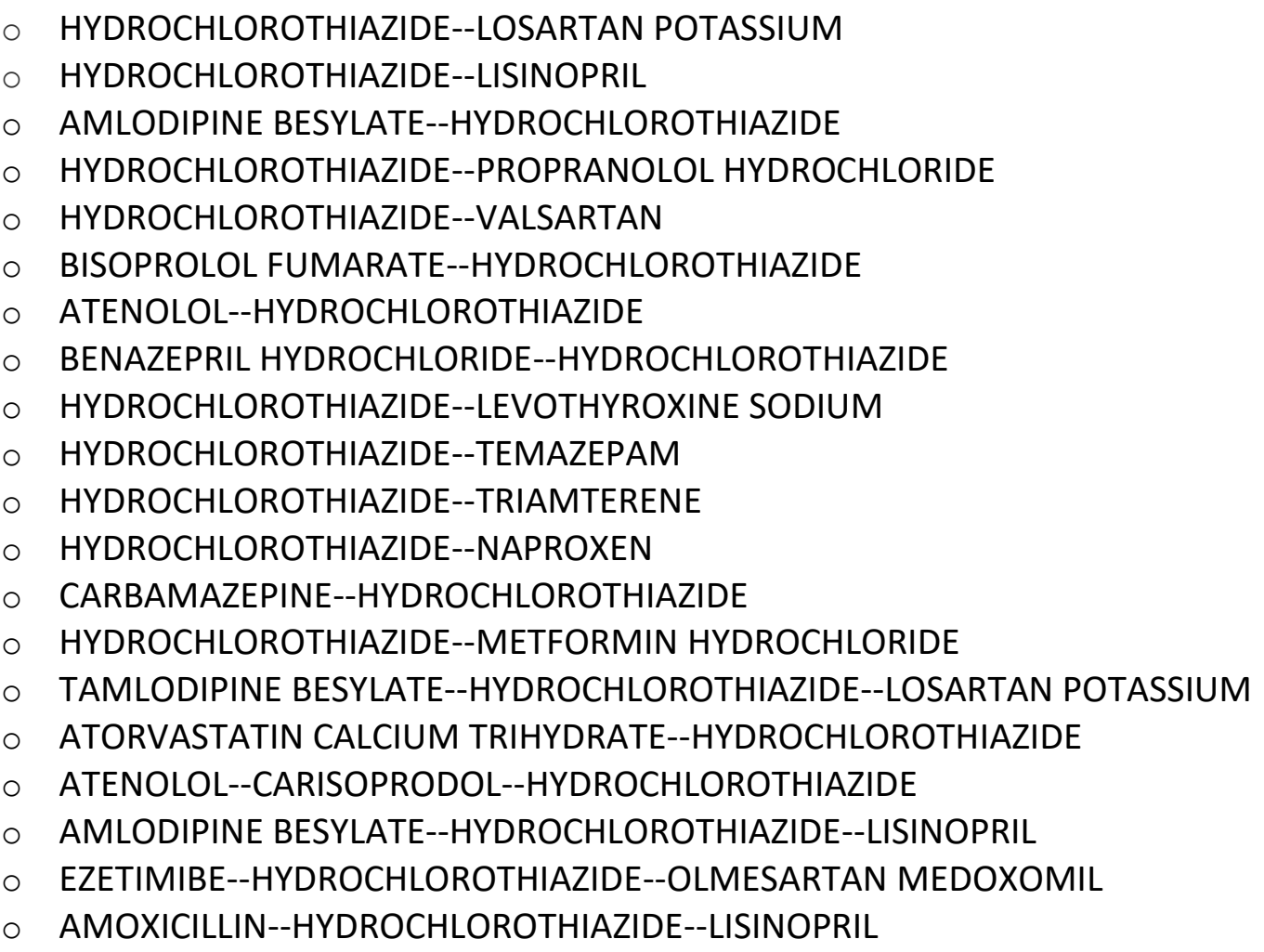


- HYDROCHLOROTHIAZIDE--LISINOPRIL--METOPROLOL TARTRATE

- AMLODIPINE BESYLATE--HYDROCHLOROTHIAZIDE--METOPROLOL TARTRATE

\subsubsection{Prevalence using number of drug classes}

Table 4.11 shows some statistics on of polypharmacy based on drug classes extracted from MEPS five years (2011-2105). Overall, the averages for number of TC1 class polypharmacy is around 2.3 while it is between 2.9 and 3 for TC1S1. The mean number of polypharmacy per patient is 2 for both types of classes. The highest number for TC1 polypharmacy elements is 13 classes. For TC1S1, there are 26 classes at most. The proportion of $\mathrm{TCl}$ single classes is almost $40 \%$ of all polypharmacy whereas it is almost $36 \%$ for TC1S1 class definition. Approximately $23 \%$ out of all polypharmacy sets are for the polypharmacy of two-TC1 classes and $19 \%$ for the polypharmacy of two-TC1S1 classes. Finally, the polypharmacy of combinations of three TC1 class is around $14 \%$ all polypharmacy while it is equal to $13 \%$ for the TC1S1 type.

Tables 4.12-4.14 show the top 30 polypharmacy sets of (single classes, two- classes, and three classes) ranked by occurrence, using TC1 drug classes.

Table 4.11: Prevalence of polypharmacy using number of drug classes

\begin{tabular}{|l|c|c|c|c|c|c|}
\hline TC1 class & $\mathbf{2 0 1 1}$ & $\mathbf{2 0 1 2}$ & $\mathbf{2 0 1 3}$ & $\mathbf{2 0 1 4}$ & $\mathbf{2 0 1 5}$ & Total \\
\hline Avg of TC1 classes in PP & 2.298 & 2.280 & 2.356 & 2.414 & 2.422 & 2.354 \\
\hline Number of PP for all patients & 39205 & 41121 & 40130 & 38839 & 40382 & 199677 \\
\hline Avg number of PP per patient & 2.045 & 2.013 & 2.010 & 2.048 & 2.060 & 2.035 \\
\hline Max number of elements in PP & 13 & 12 & 13 & 13 & 13 & 12.8 \\
\hline $\begin{array}{l}\text { Max number of polypharmacy } \\
\text { per patient }\end{array}$ & 3 & 3 & 3 & 3 & 3 & 3 \\
\hline Count of single TC1 classes & 16,467 & 16,710 & 15,742 & 15,546 & 15,345 & 79,810 \\
\hline Count of two- TC1 Classes & 9,674 & 9,887 & 9,370 & 9,309 & 9,432 & 47,672 \\
\hline Count of three-TC1 classes & 5,663 & 5,550 & 5,727 & 5,781 & 5,623 & 28,344 \\
\hline & & & & & & \\
\hline TC1S1 class & $\mathbf{2 0 1 1}$ & $\mathbf{2 0 1 2}$ & $\mathbf{2 0 1 3}$ & $\mathbf{2 0 1 4}$ & $\mathbf{2 0 1 5}$ & Total \\
\hline Avg of TC1S1 classes in PP & 2.914 & 2.891 & 3.006 & 3.096 & 3.105 & 3.002 \\
\hline Number of PP for all patients & 39205 & 41121 & 40130 & 38839 & 40382 & 199677 \\
\hline Avg number of PP per patient & 2.045 & 2.013 & 2.010 & 2.048 & 2.060 & 2.035 \\
\hline Max number of elements in PP & 23 & 23 & 24 & 26 & 23 & 23.8 \\
\hline $\begin{array}{l}\text { Max number of polypharmacy } \\
\text { per patient }\end{array}$ & 3 & 3 & 3 & 3 & 3 & 3 \\
\hline Count of single TC1S1 classes & 14,641 & 15,441 & 14,451 & 13,778 & 13,569 & 71,880 \\
\hline Count of two-TC1S1 classes & 7,811 & 8,289 & 7,831 & 7,465 & 7,514 & 38,910 \\
\hline Count of three- TC1S1 Classes & 5189 & 5473 & 5439 & 5136 & 5062 & 26299 \\
\hline
\end{tabular}


Table 4.12: Single TC1-class polypharmacy ranked by their occurrences

\begin{tabular}{|c|c|c|c|c|c|c|c|}
\hline & & \multicolumn{5}{|c|}{ Number of Occurrence } & \multirow[b]{2}{*}{ Total } \\
\hline & Single TC1-Classes & 2011 & 2012 & 2013 & 2014 & 2015 & \\
\hline 1 & $\begin{array}{l}\text { Central nervous system } \\
\text { agents }\end{array}$ & 3,329 & 3,440 & 3,386 & 3,583 & 3,422 & 17,160 \\
\hline 2 & Anti-infectives & 2,880 & 2,874 & 2,743 & 2,667 & 2,331 & 13,495 \\
\hline 3 & Cardiovascular agents & 2,257 & 2,326 & 2,116 & 2,051 & 2,133 & 10,883 \\
\hline 4 & $\begin{array}{l}\text { Hormones/hormone } \\
\text { modifiers }\end{array}$ & 1,851 & 1,867 & 1,737 & 1,707 & 1,625 & 8,787 \\
\hline 5 & Topical agents & 1,276 & 1,270 & 1,288 & 1,280 & 1,474 & 6,588 \\
\hline 6 & Respiratory agents & 1,403 & 1,373 & 1,338 & 1,128 & 1,165 & 6,407 \\
\hline 7 & Metabolic agents & 1,377 & 1,313 & 1,079 & 1,042 & 1,103 & 5,914 \\
\hline 8 & Psychotherapeutic agents & 679 & 744 & 674 & 620 & 662 & 3,379 \\
\hline 9 & Gastrointestinal agents & 512 & 569 & 473 & 482 & 525 & 2,561 \\
\hline 10 & Nutritional products & 447 & 444 & 459 & 482 & 426 & 2,258 \\
\hline 11 & Antineoplastics & 99 & 109 & 94 & 100 & 118 & 520 \\
\hline 12 & Miscellaneous agents & 82 & 104 & 77 & 84 & 77 & 424 \\
\hline 13 & Coagulation modifiers & 91 & 71 & 45 & 68 & 57 & 332 \\
\hline 14 & Genitourinary tract agents & 48 & 38 & 34 & 27 & 25 & 172 \\
\hline 15 & Immunologic agents & 17 & 32 & 30 & 34 & 24 & 137 \\
\hline 16 & Alternative medicines & 7 & 9 & 14 & 15 & 12 & 57 \\
\hline \multirow[t]{2}{*}{17} & Biologicals & 2 & 1 & & & 1 & 4 \\
\hline & Grand Total & 16,467 & 16,710 & 15,742 & 15,546 & 15,345 & 79,810 \\
\hline
\end{tabular}

The central nervous system agents have the highest occurrence for drug classification TC1.These include narcotic analgesics (pain relievers), nonnarcotic analgesics (such as acetaminophen and NSAIDs), and sedatives. 
Table 4.13: Top 30 two TC1-class polypharmacy ranked by their occurrences

\begin{tabular}{|c|c|c|c|c|c|c|c|}
\hline & & \multicolumn{5}{|c|}{ Number of Occurrence } & \multirow[b]{2}{*}{ Total } \\
\hline & Two-TC1 Classes & 2011 & 2012 & 2013 & 2014 & 2015 & \\
\hline 1 & Cardiovascular agents--Metabolic agents & 1,771 & 1,850 & 1,624 & 1,484 & 1,495 & 8,224 \\
\hline 2 & $\begin{array}{l}\text { Anti-infectives--Central nervous system } \\
\text { agents }\end{array}$ & 718 & 775 & 761 & 781 & 727 & 3,762 \\
\hline 3 & $\begin{array}{l}\text { Central nervous system agents-- } \\
\text { Psychotherapeutic agents }\end{array}$ & 626 & 613 & 545 & 675 & 613 & 3,072 \\
\hline 4 & Anti-infectives --Respiratory agents & 443 & 387 & 385 & 348 & 330 & 1,893 \\
\hline 5 & Anti-infectives--Topical agents & 369 & 375 & 395 & 350 & 311 & 1,800 \\
\hline 6 & $\begin{array}{l}\text { Central nervous system agents-- } \\
\text { Cardiovascular agents }\end{array}$ & 328 & 340 & 305 & 353 & 337 & 1,663 \\
\hline 7 & $\begin{array}{l}\text { Central nervous system agents-- } \\
\text { Hormones/hormone modifiers }\end{array}$ & 287 & 299 & 236 & 309 & 330 & 1,461 \\
\hline 8 & $\begin{array}{l}\text { Cardiovascular agents--Central nervous } \\
\text { system agents }\end{array}$ & 236 & 292 & 277 & 294 & 281 & 1,380 \\
\hline 9 & $\begin{array}{l}\text { Cardiovascular agents-- } \\
\text { Hormones/hormone modifiers }\end{array}$ & 287 & 292 & 233 & 241 & 282 & 1,335 \\
\hline 10 & $\begin{array}{l}\text { Central nervous system agents-- } \\
\text { Respiratory agents }\end{array}$ & 276 & 306 & 264 & 210 & 219 & 1,275 \\
\hline 11 & Respiratory agents--Topical agents & 250 & 225 & 258 & 219 & 245 & 1,197 \\
\hline 12 & $\begin{array}{l}\text { Central nervous system agents--Metabolic } \\
\text { agents }\end{array}$ & 279 & 234 & 238 & 219 & 226 & 1,196 \\
\hline 13 & $\begin{array}{l}\text { Central nervous system agents--Topical } \\
\text { agents }\end{array}$ & 210 & 199 & 222 & 204 & 276 & 1,111 \\
\hline 14 & $\begin{array}{l}\text { Central nervous system agents-- } \\
\text { Gastrointestinal agents }\end{array}$ & 200 & 202 & 218 & 240 & 231 & 1,091 \\
\hline 15 & $\begin{array}{l}\text { Anti-infectives--Hormones/hormone } \\
\text { modifiers }\end{array}$ & 217 & 205 & 215 & 213 & 225 & 1,075 \\
\hline 16 & $\begin{array}{l}\text { Hormones/hormone modifiers-- } \\
\text { Respiratory agents }\end{array}$ & 179 & 170 & 169 & 173 & 144 & 835 \\
\hline 17 & $\begin{array}{l}\text { Cardiovascular agents--Gastrointestinal } \\
\text { agents }\end{array}$ & 156 & 170 & 152 & 176 & 176 & 830 \\
\hline 18 & $\begin{array}{l}\text { Cardiovascular agents--Psychotherapeutic } \\
\text { agents }\end{array}$ & 140 & 169 & 152 & 130 & 161 & 752 \\
\hline 19 & Anti-infectives--Cardiovascular agents & 139 & 151 & 140 & 162 & 144 & 736 \\
\hline 20 & Cardiovascular agents--Topical agents & 143 & 108 & 114 & 123 & 163 & 651 \\
\hline 21 & $\begin{array}{l}\text { Hormones/hormone modifiers--Topical } \\
\text { agents }\end{array}$ & 108 & 137 & 115 & 129 & 142 & 631 \\
\hline 22 & $\begin{array}{l}\text { Hormones/hormone modifiers--Metabolic } \\
\text { agents }\end{array}$ & 119 & 148 & 126 & 100 & 125 & 618 \\
\hline 23 & $\begin{array}{l}\text { Central nervous system agents-- } \\
\text { Nutritional products }\end{array}$ & 105 & 121 & 139 & 127 & 110 & 602 \\
\hline 24 & $\begin{array}{l}\text { Hormones/hormone modifiers-- } \\
\text { Psychotherapeutic agents }\end{array}$ & 145 & 125 & 98 & 103 & 106 & 577 \\
\hline
\end{tabular}




\begin{tabular}{|c|l|c|c|c|c|c|c|}
\hline 25 & Cardiovascular agents--Respiratory agents & 99 & 90 & 117 & 90 & 108 & 504 \\
\hline 26 & $\begin{array}{l}\text { Cardiovascular agents--Nutritional } \\
\text { products }\end{array}$ & 95 & 99 & 108 & 102 & 97 & 501 \\
\hline 27 & Gastrointestinal agents--Metabolic agents & 85 & 88 & 90 & 75 & 101 & 439 \\
\hline 28 & $\begin{array}{l}\text { Metabolic agents --Psychotherapeutic } \\
\text { agents }\end{array}$ & 75 & 78 & 90 & 68 & 67 & 378 \\
\hline 29 & Metabolic agents --Topical agents & 78 & 77 & 67 & 66 & 76 & 364 \\
\hline 30 & Anti-infectives-Metabolic agents & 85 & 67 & 76 & 61 & 58 & 347 \\
\hline
\end{tabular}

The most common class pair using TC1 classification is (Cardiovascular agents- Metabolic agents). Cardiovascular agents are used to treat medical conditions for heart or the circulatory system (blood vessels). Some instances are arrhythmias, blood clots, coronary artery disease, high or low blood pressure, high cholesterol, heart failure, and stroke. Metabolic agents are used to treat metabolic disorders. These disorders are defects of metabolism which occur when the body is not able to properly process fats, proteins, sugars, or nucleic acids.

Table 4.14: Top 30 three TC1-class polypharmacy ranked by their occurrences

\begin{tabular}{|c|l|c|c|c|c|c|c|}
\hline & & \multicolumn{5}{|c|}{ Number of Occurrence } & \\
\hline & Three-TC1 Classes & $\mathbf{2 0 1 1}$ & $\mathbf{2 0 1 2}$ & $\mathbf{2 0 1 3}$ & $\mathbf{2 0 1 4}$ & $\mathbf{2 0 1 5}$ & Total \\
\hline 1 & $\begin{array}{l}\text { Cardiovascular agents--Central nervous system } \\
\text { agents-- Metabolic agents }\end{array}$ & 290 & 282 & 226 & 285 & 293 & 1376 \\
\hline 2 & $\begin{array}{l}\text { Cardiovascular agents--Hormones/hormone } \\
\text { modifiers-- Metabolic agents }\end{array}$ & 241 & 261 & 241 & 203 & 213 & 1159 \\
\hline 3 & $\begin{array}{l}\text { Central nervous system agents--Cardiovascular } \\
\text { agents-- Metabolic agents }\end{array}$ & 226 & 254 & 212 & 253 & 212 & 1157 \\
\hline 4 & $\begin{array}{l}\text { Cardiovascular agents--Gastrointestinal agents-- } \\
\text { Metabolic agents }\end{array}$ & 206 & 204 & 214 & 194 & 220 & 1038 \\
\hline 5 & $\begin{array}{l}\text { Cardiovascular agents-- Metabolic agents -- } \\
\text { Topical agents }\end{array}$ & 127 & 134 & 115 & 150 & 126 & 652 \\
\hline 6 & $\begin{array}{l}\text { Central nervous system agents--Cardiovascular } \\
\text { agents--Psychotherapeutic agents }\end{array}$ & 117 & 123 & 110 & 141 & 157 & 648 \\
\hline 7 & $\begin{array}{l}\text { Cardiovascular agents-- Metabolic agents -- } \\
\text { Nutritional products }\end{array}$ & 138 & 118 & 130 & 133 & 103 & 622 \\
\hline 8 & $\begin{array}{l}\text { Cardiovascular agents-- Metabolic agents -- } \\
\text { Psychotherapeutic agents }\end{array}$ & 115 & 128 & 120 & 94 & 110 & 567 \\
\hline 9 & $\begin{array}{l}\text { Anti-infectives--Central nervous system agents-- } \\
\text { Respiratory agents }\end{array}$ & 129 & 87 & 138 & 104 & 82 & 540 \\
\hline 10 & $\begin{array}{l}\text { Cardiovascular agents--Coagulation modifiers-- } \\
\text { Metabolic agents }\end{array}$ & 114 & 122 & 100 & 108 & 81 & 525 \\
\hline 11 & $\begin{array}{l}\text { Anti-infectives--Cardiovascular agents-- } \\
\text { Metabolic agents }\end{array}$ & 96 & 102 & 114 & 105 & 73 & 490 \\
\hline 12 & $\begin{array}{l}\text { Anti-infectives--Central nervous system agents-- } \\
\text { Topical agents }\end{array}$ & 87 & 77 & 108 & 98 & 119 & 489 \\
\hline 13 & $\begin{array}{l}\text { Anti-infectives--Hormones/hormone modifiers-- } \\
\text { Respiratory agents }\end{array}$ & 104 & 100 & 77 & 108 & 84 & 473 \\
\hline
\end{tabular}




\begin{tabular}{|c|c|c|c|c|c|c|c|}
\hline 14 & $\begin{array}{l}\text { Central nervous system agents-- } \\
\text { Hormones/hormone modifiers-- } \\
\text { Psychotherapeutic agents }\end{array}$ & 86 & 109 & 99 & 85 & 94 & 473 \\
\hline 15 & $\begin{array}{l}\text { Anti-infectives--Respiratory agents--Topical } \\
\text { agents }\end{array}$ & 88 & 78 & 107 & 84 & 88 & 445 \\
\hline 16 & $\begin{array}{l}\text { Anti-infectives--Central nervous system agents-- } \\
\text { Hormones/hormone modifiers }\end{array}$ & 97 & 78 & 86 & 84 & 94 & 439 \\
\hline 17 & $\begin{array}{l}\text { Anti-infectives--Central nervous system agents-- } \\
\text { Psychotherapeutic agents }\end{array}$ & 83 & 71 & 88 & 83 & 84 & 409 \\
\hline 18 & $\begin{array}{l}\text { Cardiovascular agents--Central nervous system } \\
\text { agents--Psychotherapeutic agents }\end{array}$ & 80 & 84 & 65 & 82 & 75 & 386 \\
\hline 19 & $\begin{array}{l}\text { Cardiovascular agents-- Metabolic agents -- } \\
\text { Respiratory agents }\end{array}$ & 91 & 63 & 65 & 71 & 92 & 382 \\
\hline 20 & $\begin{array}{l}\text { Coagulation modifiers--Cardiovascular agents-- } \\
\text { Metabolic agents }\end{array}$ & 85 & 82 & 74 & 63 & 71 & 375 \\
\hline 21 & $\begin{array}{l}\text { Central nervous system agents-- Metabolic } \\
\text { agents--Psychotherapeutic agents }\end{array}$ & 52 & 73 & 60 & 83 & 51 & 319 \\
\hline 22 & $\begin{array}{l}\text { Central nervous system agents--Respiratory } \\
\text { agents--Topical agents }\end{array}$ & 59 & 62 & 65 & 66 & 58 & 310 \\
\hline 23 & $\begin{array}{l}\text { Central nervous system agents--Gastrointestinal } \\
\text { agents--Psychotherapeutic agents }\end{array}$ & 53 & 61 & 69 & 62 & 62 & 307 \\
\hline 24 & $\begin{array}{l}\text { Central nervous system agents--Cardiovascular } \\
\text { agents--Gastrointestinal agents }\end{array}$ & 48 & 57 & 61 & 73 & 66 & 305 \\
\hline 25 & $\begin{array}{l}\text { Anti-infectives--Central nervous system agents-- } \\
\text { Cardiovascular agents }\end{array}$ & 55 & 57 & 59 & 66 & 46 & 283 \\
\hline 26 & $\begin{array}{l}\text { Anti-infectives--Central nervous system agents-- } \\
\text { Gastrointestinal agents }\end{array}$ & 40 & 39 & 70 & 58 & 75 & 282 \\
\hline 27 & $\begin{array}{l}\text { Central nervous system agents-- } \\
\text { Psychotherapeutic agents--Respiratory agents }\end{array}$ & 57 & 35 & 62 & 64 & 53 & 271 \\
\hline 28 & $\begin{array}{l}\text { Central nervous system agents--Cardiovascular } \\
\text { agents--Hormones/hormone modifiers }\end{array}$ & 47 & 47 & 48 & 49 & 52 & 243 \\
\hline 29 & $\begin{array}{l}\text { Anti-infectives-Hormones/hormone modifiers- } \\
\text { Topical agents }\end{array}$ & 45 & 48 & 50 & 37 & 55 & 235 \\
\hline 30 & $\begin{array}{l}\text { Cardiovascular agents-Central nervous system } \\
\text { agents-Gastrointestinal agents }\end{array}$ & 48 & 40 & 66 & 42 & 32 & 228 \\
\hline
\end{tabular}

As indicated earlier in Chapter 3, using drug classes will provide less specific information on the association with health problems. This could be seen by observing Table 4.12 that lists all single TC1-class polypharmacy ranked by their occurrences, and Table 7 (in Appendix A) that lists of all single TC1-class polypharmacy ranked by the corresponding ER visits. Even though the latter is a ranking based on a health problem, the order for these two tables were quite similar. This could be misleading since drugs in the same class work on multiple targets and thus we emphasis that using drugs or active ingredients provide a more accurate approaches to define polypharmacy. Next Tables 4.15 - 4.17 include the top 30 single TC1S1-classes, as well as the pairs and triples defined using TC1S1 approach. 
Table 4.15: Top 30 single TC1S1-classes in the dataset ranked by their occurrences

\begin{tabular}{|c|c|c|c|c|c|c|c|}
\hline & & \multicolumn{5}{|c|}{ Number of Occurrence } & \multirow[b]{2}{*}{ Total } \\
\hline & Single TC1S1 Classes & 2011 & 2012 & 2013 & 2014 & 2015 & \\
\hline 1 & ANALGESICS & 1,652 & 1,773 & 1,759 & 1,722 & 1,613 & 8,519 \\
\hline 2 & PENICILLINS & 1,168 & 1,151 & 1,090 & 1,034 & 1,150 & 5,593 \\
\hline 3 & SEX_HORMONES & 1,072 & 1,102 & 994 & 905 & 872 & 4,945 \\
\hline 4 & MACROLIDE_DERIVATIVES & 781 & 901 & 742 & 716 & 217 & 3,357 \\
\hline 5 & DERMATOLOGICAL_AGENTS & 644 & 641 & 620 & 619 & 802 & 3,326 \\
\hline 6 & CNS_STIMULANTS & 557 & 586 & 562 & 635 & 619 & 2,959 \\
\hline 7 & ANTIHYPERLIPIDEMIC_AGENTS & 721 & 646 & 525 & 498 & 541 & 2,931 \\
\hline 8 & ANTIDEPRESSANTS & 558 & 630 & 552 & 512 & 546 & 2,798 \\
\hline 9 & BRONCHODILATORS & 558 & 597 & 546 & 466 & 516 & 2,683 \\
\hline 10 & THYROID_HORMONES & 497 & 538 & 503 & 496 & 508 & 2,542 \\
\hline 11 & ANTIDIABETIC_AGENTS & 444 & 481 & 396 & 362 & 396 & 2,079 \\
\hline 12 & ANGIOTENSIN_CONVERTING_ENZYME_INHIBITORS & 394 & 459 & 407 & 372 & 373 & 2,005 \\
\hline 13 & ANTIHYPERTENSIVE_COMBINATIONS & 423 & 439 & 401 & 340 & 322 & 1,925 \\
\hline 14 & OPHTHALMIC_PREPARATIONS & 301 & 345 & 351 & 351 & 388 & 1,736 \\
\hline 15 & PROTON_PUMP_INHIBITORS & 278 & 328 & 274 & 256 & 293 & 1,429 \\
\hline 16 & ANTICONVULSANTS & 241 & 310 & 253 & 243 & 233 & 1,280 \\
\hline 18 & BETA-ADRENERGIC_BLOCKING_AGENTS & 307 & 266 & 181 & 181 & 168 & 1,103 \\
\hline 19 & IRON_PRODUCTS & 218 & 211 & 245 & 216 & 123 & 1,013 \\
\hline 20 & ANTIHISTAMINES & 205 & 181 & 141 & 199 & 195 & 921 \\
\hline 21 & CEPHALOSPORINS & 186 & 173 & 191 & 172 & 149 & 871 \\
\hline 22 & NASAL_PREPARATIONS & 188 & 166 & 215 & 175 & 121 & 865 \\
\hline 23 & UPPER_RESPIRATORY_COMBINATIONS & 177 & 161 & 243 & 114 & 127 & 822 \\
\hline 24 & CALCIUM_CHANNEL_BLOCKING_AGENTS & 160 & 148 & 147 & 141 & 208 & 804 \\
\hline 25 & ADRENAL_CORTICAL_STEROIDS & 156 & 158 & 164 & 179 & 124 & 781 \\
\hline 26 & ANTIVIRAL_AGENTS & 120 & 140 & 170 & 158 & 152 & 740 \\
\hline 27 & ANTIEMETIC/ANTIVERTIGO_AGENTS & 131 & 148 & 159 & 149 & 137 & 724 \\
\hline 28 & DIURETICS & 151 & 146 & 127 & 147 & 145 & 716 \\
\hline 29 & ANXIOLYTICSSEDATIVESAND_HYPNOTICS & 125 & 143 & 134 & 131 & 104 & 637 \\
\hline 30 & ANGIOTENSIN_II_INHIBITORS & 90 & 117 & 114 & 103 & 155 & 579 \\
\hline
\end{tabular}


Table 4.16: Top 30 two-TC1S1 classes polypharmacy ranked by their occurrences

\begin{tabular}{|c|c|c|c|c|c|c|c|}
\hline & & Numb & er of 0 & curren & & & \\
\hline & Two-TC1S1 Classes & 2011 & 2012 & 2013 & 2014 & 2015 & Total \\
\hline 1 & ANALGESICS--PENICILLINS & 195 & 216 & 245 & 245 & 268 & 1,169 \\
\hline 2 & ANALGESICS--MUSCLE_RELAXANTS & 177 & 184 & 188 & 195 & 177 & 921 \\
\hline 3 & ANALGESICS--MACROLIDE_DERIVATIVES & 153 & 151 & 137 & 138 & 30 & 609 \\
\hline 4 & $\begin{array}{l}\text { ANTIHYPERLIPIDEMIC_AGENTS-- } \\
\text { BETA ADRENERGIC BLOCKING AGENTS }\end{array}$ & 92 & 113 & 90 & 56 & 51 & 402 \\
\hline 5 & $\begin{array}{l}\text { ANGIOTENSIN_CONVERTING_ENZYME_INHIBITORS-- } \\
\text { ANTIHYPERLIPIDEMIC AGENTS }\end{array}$ & 85 & 73 & 80 & 72 & 59 & 369 \\
\hline 6 & $\begin{array}{l}\text { ANTIHYPERTENSIVE_COMBINATIONS-- } \\
\text { ANTIHYPERLIPIDEMIC_AGENTS }\end{array}$ & 80 & 94 & 66 & 50 & 61 & 351 \\
\hline 7 & $\begin{array}{l}\text { ANTIDIABETIC_AGENTS-- } \\
\text { ANTIHYPERLIPIDEMIC_AGENTS }\end{array}$ & 63 & 83 & 68 & 68 & 56 & 338 \\
\hline 8 & ANALGESICS--DERMATOLOGICAL_AGENTS & 57 & 51 & 53 & 55 & 106 & 322 \\
\hline 9 & $\begin{array}{l}\text { ADRENAL_CORTICAL_STEROIDS-- } \\
\text { BRONCHODILATORS }\end{array}$ & 66 & 68 & 71 & 65 & 49 & 319 \\
\hline 10 & $\begin{array}{l}\text { BRONCHODILATORS-- } \\
\text { RESPIRATORY_INHALANT_PRODUCTS }\end{array}$ & 73 & 82 & 68 & 45 & 40 & 308 \\
\hline 11 & $\begin{array}{l}\text { ANTIDIABETIC_AGENTS-- } \\
\text { ANGIOTENSIN_CONVERTING_ENZYME_INHIBITORS }\end{array}$ & 66 & 66 & 60 & 57 & 50 & 299 \\
\hline 12 & $\begin{array}{l}\text { ANTIHYPERLIPIDEMIC_AGENTS-- } \\
\text { ANTIHYPERTENSIVE_COMBINATIONS }\end{array}$ & 56 & 64 & 65 & 54 & 57 & 296 \\
\hline 13 & $\begin{array}{l}\text { ANGIOTENSIN_CONVERTING_ENZYME_INHIBITORS-- } \\
\text { DIURETICS }\end{array}$ & 58 & 64 & 46 & 57 & 58 & 283 \\
\hline 14 & ANALGESICS--ANTICONVULSANTS & 48 & 61 & 39 & 55 & 78 & 281 \\
\hline 15 & $\begin{array}{l}\text { ANTIHYPERLIPIDEMIC_AGENTS-- } \\
\text { ANGIOTENSIN_CONVERTING_ENZYME_INHIBITORS }\end{array}$ & 57 & 67 & 69 & 52 & 35 & 280 \\
\hline 16 & ANALGESICS--SEX_HORMONES & 59 & 60 & 51 & 57 & 51 & 278 \\
\hline 17 & ANTIDEPRESSANTS--CNS_STIMULANTS & 33 & 61 & 43 & 71 & 60 & 268 \\
\hline 18 & BRONCHODILATORS--LEUKOTRIENE_MODIFIERS & 72 & 59 & 53 & 38 & 41 & 263 \\
\hline 19 & ANALGESICS--PROTON_PUMP_INHIBITORS & 55 & 41 & 46 & 58 & 60 & 260 \\
\hline 20 & ANTIDEPRESSANTS--SEX_HORMONES & 57 & 54 & 36 & 53 & 52 & 252 \\
\hline 21 & SEX_HORMONES--THYROID_HORMONES & 48 & 64 & 54 & 39 & 37 & 242 \\
\hline 22 & $\begin{array}{l}\text { ANGIOTENSIN_CONVERTING_ENZYME_INHIBITORS-- } \\
\text { BETA_ADRENERGIC_BLOCKING_AGENTS }\end{array}$ & 46 & 49 & 48 & 48 & 40 & 231 \\
\hline 23 & $\begin{array}{l}\text { ANGIOTTENSIN_CONVEERTING_ENZ̄YYME_INHIBITORS-- } \\
\text { CALCIUM_CHANNEL_BLOCKING_AGENTS }\end{array}$ & 41 & 49 & 40 & 48 & 51 & 229 \\
\hline 24 & ANALGESICS--CEPHALLOSPORINS & 49 & 41 & 37 & 58 & 38 & 223 \\
\hline 25 & $\begin{array}{l}\text { ANTIHYPERLIPIDEMIC_AGENTS-- } \\
\text { THYROID_HORMONES }\end{array}$ & 33 & 51 & 54 & 36 & 49 & 223 \\
\hline 26 & ANTIDEPRESSANTS--THYROID_HORMONES & 51 & 55 & 39 & 32 & 41 & 218 \\
\hline 27 & $\begin{array}{l}\text { ANTIHYPERLIPIDEMIC_AGENTS-- } \\
\text { PROTON_PUMP_INHIBITORS }\end{array}$ & 49 & 37 & 44 & 36 & 52 & 218 \\
\hline 28 & $\begin{array}{l}\text { ANTIADRENERGIC_AGENTSCENTRALLY_ACTING-- } \\
\text { CNS_STIMULANTS }\end{array}$ & 44 & 42 & 40 & 51 & 34 & 211 \\
\hline 29 & BRONCHODILATORS--PENICILLINS & 44 & 46 & 40 & 40 & 40 & 210 \\
\hline 30 & DERMATOLOGICAL_AGENTS--PENICILLINS & 39 & 40 & 36 & 43 & 47 & 205 \\
\hline
\end{tabular}


In general, the two-class polypharmacy of TC1S1 have lower occurrences than the TC1 two-classes polypharmacy. This is expected given the much larger number of TC1S1 classes.

Table 4.17: Top 30 three-TC1S1 classes polypharmacy ranked by their occurrences

\begin{tabular}{|c|c|c|c|c|c|c|c|}
\hline & \multirow[b]{2}{*}{ Three-TC1S1 Classes } & \multicolumn{5}{|c|}{ Number of Occurrence } & \multirow[b]{2}{*}{ Tota } \\
\hline & & 2011 & 2012 & 2013 & 2014 & 2015 & \\
\hline 1 & $\begin{array}{l}\text { ANTIDIABETIC_AGENTS-- } \\
\text { ANGIOTENSIN_CONVERTING_ENZYME_INHIBITORS-- } \\
\text { ANTIHYPERLIPIDEMIC_AGENTS }\end{array}$ & 39 & 37 & 39 & 33 & 37 & 185 \\
\hline 2 & $\begin{array}{l}\text { ANTIDIABETIC_AGENTS-- } \\
\text { ANTIHYPERLIPIDEMIC_AGENTS-- } \\
\text { ANGIOTENSIN_CONVERTING_ENZYME_INHIBITORS }\end{array}$ & 25 & 30 & 28 & 35 & 39 & 157 \\
\hline 3 & $\begin{array}{l}\text { ANGIOTENSIN_CONVERTING_ENZYME_INHIBITORS-- } \\
\text { ANTIHYPERLIPIDEMIC_AGENTS-- } \\
\text { BETA_ADRENERGIC_BLOCKING_AGENTS }\end{array}$ & 30 & 31 & 16 & 23 & 16 & 116 \\
\hline 4 & $\begin{array}{l}\text { ANGIOTENSIN_CONVERTING_ENZYME_INHIBITORS-- } \\
\text { BETA_ADRENERGIC_BLOCKING_AGENTS--DIURETICS }\end{array}$ & 13 & 16 & 24 & 17 & 16 & 86 \\
\hline 5 & $\begin{array}{l}\text { ANTIHYPERLIPIDEMIC_AGENTS-- } \\
\text { ANGIOTENSIN_CONVERTING_ENZYME_INHIBITORS-- } \\
\text { ANTIDIABETIC_AGENTS }\end{array}$ & 13 & 21 & 11 & 21 & 17 & 83 \\
\hline 6 & $\begin{array}{l}\text { ANTIHYPERLIPIDEMIC_AGENTS-- } \\
\text { BETA_ADRENERGIC_BLOCKING_AGENTS--DIURETICS }\end{array}$ & 16 & 31 & 18 & 7 & 10 & 82 \\
\hline 7 & $\begin{array}{l}\text { ANGIOTENSIN_CONVERTING_ENZYME_INHIBITORS-- } \\
\text { ANTIHYPERLIPIDEMIC_AGENTS-- } \\
\text { ANTIDIABETIC_AGENTS }\end{array}$ & 9 & 17 & 10 & 15 & 29 & 80 \\
\hline 8 & $\begin{array}{l}\text { ANTIHYPERTENSIVE_COMBINATIONS-- } \\
\text { ANTIHYPERLIPIDEMIC_AGENTS-- } \\
\text { BETA_ADRENERGIC_BLOCKING_AGENTS }\end{array}$ & 28 & 14 & 15 & 8 & 13 & 78 \\
\hline 9 & $\begin{array}{l}\text { ADRENAL_CORTICAL_STEROIDS-- } \\
\text { BRONCHODILATORS--MACROLIDE_DERIVATIVES }\end{array}$ & 17 & 20 & 10 & 19 & 11 & 77 \\
\hline 10 & $\begin{array}{l}\text { ANTIHYPERLIPIDEMIC_AGENTS-- } \\
\text { BETA_ADRENERGIC_BLOCKING_AGENTS-- } \\
\text { CALCIUM_CHANNEL_BLOCKING_AGENTS }\end{array}$ & 18 & 17 & 8 & 15 & 14 & 72 \\
\hline 11 & $\begin{array}{l}\text { ANGIOTENSIN_CONVERTING_ENZYME_INHIBITORS-- } \\
\text { ANTIHYPERLIPIDEMIC_AGENTS--DIURETICS }\end{array}$ & 13 & 17 & 14 & 14 & 12 & 70 \\
\hline 12 & $\begin{array}{l}\text { ANTIHYPERLIPIDEMIC_AGENTS-- } \\
\text { ANGIOTENSIN_CONVERTING_ENZYME_INHIBITORS-- } \\
\text { BETA_ADRENERGIC_BLOCKING_AGENTS }\end{array}$ & 20 & 14 & 10 & 13 & 13 & 70 \\
\hline 13 & $\begin{array}{l}\text { ANALGESICS--ANTICONVULSANTS-- } \\
\text { MUSCLE_RELAXANTS }\end{array}$ & 13 & 10 & 13 & 11 & 19 & 66 \\
\hline 14 & $\begin{array}{l}\text { ANALGESICS--PENICILLINS-- } \\
\text { UPPER_RESPIRATORY_COMBINATIONS }\end{array}$ & 18 & 11 & 16 & 15 & 6 & 66 \\
\hline 15 & $\begin{array}{l}\text { BRONCHODILATORS--LEUKOTRIENE_MODIFIERS-- } \\
\text { RESPIRATORY_INHALANT_PRODUCTS }\end{array}$ & 19 & 10 & 17 & 13 & 3 & 62 \\
\hline
\end{tabular}




\begin{tabular}{|c|c|c|c|c|c|c|c|}
\hline 16 & $\begin{array}{l}\text { ANTIDIABETIC_AGENTS-- } \\
\text { ANTIHYPERTENSIVE_COMBINATIONS-- } \\
\text { ANTIHYPERLIPIDEMIC_AGENTS }\end{array}$ & 12 & 11 & 13 & 14 & 8 & 58 \\
\hline 17 & $\begin{array}{l}\text { ADRENAL_CORTICAL_STEROIDS-- } \\
\text { BRONCHODILATORS--PENICILLINS }\end{array}$ & 11 & 16 & 7 & 16 & 6 & 56 \\
\hline 18 & $\begin{array}{l}\text { ANTIHYPERLIPIDEMIC_AGENTS-- } \\
\text { CALCIUM_CHANNEL_BLOCKING_AGENTS--DIURETICS }\end{array}$ & 21 & 14 & 9 & 6 & 6 & 56 \\
\hline 19 & $\begin{array}{l}\text { ANTIHYPERLIPIDEMIC_AGENTS-- } \\
\text { BETA_ADRENERGIC_BLOCKING_AGENTS-- } \\
\text { PROTON_PUMP_INHIBITORS }\end{array}$ & 13 & 12 & 6 & 10 & 12 & 53 \\
\hline 20 & $\begin{array}{l}\text { ANGIOTENSIN_CONVERTING_ENZYME_INHIBITORS-- } \\
\text { ANTIHYPERLIPIDEMIC_AGENTS-- } \\
\text { THYROID_HORMONES }\end{array}$ & 8 & 5 & 9 & 20 & 10 & 52 \\
\hline 21 & $\begin{array}{l}\text { ANTIHYPERTENSIVE_COMBINATIONS-- } \\
\text { ANTIHYPERLIPIDEMIC_AGENTS-- } \\
\text { THYROID_HORMONES }\end{array}$ & 7 & 10 & 13 & 11 & 11 & 52 \\
\hline 22 & $\begin{array}{l}\text { ANGIOTENSIN_CONVERTING_ENZYME_INHIBITORS-- } \\
\text { CALCIUM_CHANNEL_BLOCKING_AGENTS--DIURETICS }\end{array}$ & 13 & 12 & 19 & 6 & 2 & 52 \\
\hline 23 & $\begin{array}{l}\text { ANTIHYPERLIPIDEMIC_AGENTS-- } \\
\text { BETA_ADRENERGIC_BLOCKING_AGENTS-- } \\
\text { THYROID_HORMONES }\end{array}$ & 9 & 17 & 10 & 6 & 9 & 51 \\
\hline 24 & $\begin{array}{l}\text { ANTICONVULSANTS--ANALGESICS-- } \\
\text { MUSCLE_RELAXANTS }\end{array}$ & 3 & 12 & 10 & 11 & 12 & 48 \\
\hline 25 & $\begin{array}{l}\text { ANTIHYPERLIPIDEMIC_AGENTS-- } \\
\text { ANGIOTENSIN_CONVERTING_ENZYME_INHIBITORS-- } \\
\text { DIURETICS }\end{array}$ & 13 & 12 & 11 & 4 & 8 & 48 \\
\hline 26 & $\begin{array}{l}\text { ANALGESICS--ANTIDEPRESSANTS-- } \\
\text { MUSCLE_RELAXANTS }\end{array}$ & 11 & 14 & 10 & 7 & 4 & 46 \\
\hline 27 & $\begin{array}{l}\text { ANGIOTENSIN_II_INHIBITORS-- } \\
\text { ANTIHYPERLIPIDEMIC_AGENTS-- } \\
\text { BETA_ADRENERGIC_BLOCKING_AGENTS }\end{array}$ & 7 & 14 & 3 & 10 & 12 & 46 \\
\hline 28 & $\begin{array}{l}\text { ANTIHYPERLIPIDEMIC_AGENTS-- } \\
\text { PROTON_PUMP_INHIBITORS--THYROID_HORMONES }\end{array}$ & 13 & 9 & 6 & 6 & 12 & 46 \\
\hline 29 & $\begin{array}{l}\text { ANTIDIABETIC_AGENTS-- } \\
\text { ANGIOTENSIN_II_INHIBITORS-- } \\
\text { ANTIHYPERLIPIDEMIC_AGENTS }\end{array}$ & 10 & 11 & 12 & 10 & 2 & 45 \\
\hline 30 & $\begin{array}{l}\text { ANGIOTENSIN_CONVERTING_ENZYME_INHIBITORS-- } \\
\text { ANTIDIABETIC_AGENTS-- } \\
\text { ANTIHYPERLIPIDEMIC_AGENTS }\end{array}$ & 7 & 10 & 9 & 11 & 8 & 45 \\
\hline
\end{tabular}

In general, defining polypharmacy using the therapeutic class TC1S1 provides better associations to health problems than TC1 definition. This could be inferred by the fact that TC1S1 has more granularity than TC1 since, in contrast to the TC1 classification, it considers the ingredients of the drugs in its classification [31]. Also, TC1S1 has shown closer overall trends to those of drugs and Al as will be shown latter in Sections 4.3 and 4.5. 


\subsubsection{Prevalence summary}

The highest average number of medication elements in polypharmacy were found in polypharmacy of active ingredients with the range (3.6-3.8), followed by polypharmacy of drugs with the range (3.2-3.4), followed by polypharmacy of TC1S1 with the range (2.8-3.1), and finally the lowest average number of medication elements were found for polypharmacy of TC1 class with the range (2.2-2.4). Overall, averages of the number of medication elements have increased over time (2011-2015), which means an increase in prescriptions trend. Table 4.18 shows summary of the prevalence analysis across all definitions, and Table 4.19 summarizes the proportion of single-element, two-element, and three-element polypharmacy for each definition.

Table 4.18 Summary of prevalence 2011 -2015

\begin{tabular}{|c|c|c|c|c|}
\hline & $\begin{array}{c}\text { Prevalence of } \\
\text { Drug PP }\end{array}$ & $\begin{array}{c}\text { Prevalence of } \\
\text { Active ingredients } \\
\text { PP }\end{array}$ & $\begin{array}{c}\text { Prevalence of } \\
\text { TC1 class } \\
\text { PP }\end{array}$ & $\begin{array}{c}\text { Prevalence of } \\
\text { TC1S1 class PP }\end{array}$ \\
\hline $\begin{array}{c}\text { Avg \# of PP } \\
\text { element }\end{array}$ & 3.365 & 3.730 & 2.354 & 3.002 \\
\hline Avg \# of PP & 2.035 & 2.035 & 2.0352 & 2.035 \\
\hline Max \# elements & 34.4 & 36.6 & 12.8 & 23.8 \\
\hline $\begin{array}{c}\text { Max \# PP per } \\
\text { patient }\end{array}$ & 3 & 3 & 3 & 3 \\
\hline \#1-element & 67854 & 58,993 & 79810 & 71880 \\
\hline \#2-element & 39481 & 37840 & 47672 & 38910 \\
\hline \#3-element & 26861 & 26912 & 28344 & 26299 \\
\hline
\end{tabular}

Table 4.19 proportion of single-element, two-element, and three-element of polypharmacy of all definitions.

\begin{tabular}{|l|c|c|c|c|}
\hline & Drugs & $\begin{array}{c}\text { Active } \\
\text { Ingredients }\end{array}$ & TC1 drug class & TC1S1 drug class \\
\hline $\begin{array}{l}\text { Proportion of single- } \\
\text { element }\end{array}$ & $34 \%$ & $29 \%$ & $40 \%$ & $36 \%$ \\
\hline $\begin{array}{l}\text { Proportion of two- } \\
\text { element }\end{array}$ & $19 \%$ & $18 \%$ & $23 \%$ & $19 \%$ \\
\hline $\begin{array}{l}\text { Proportion of three- } \\
\text { elements }\end{array}$ & $13 \%$ & $13 \%$ & $14 \%$ & $13 \%$ \\
\hline
\end{tabular}




\subsection{Association of polypharmacy with ER}

This section provides detailed view of relationship between ER and the number of medication elements (drugs, Als, or drug classes) in different polypharmacy groups. To summarize these results, it was observed that measure (1) (i.e., The proportion of ER visit to the number of polypharmacy sets in a cluster (i), ERPP(i) that was defined previously in Chapter 3 ) has been increased with increased number of elements in polypharmacy for all definitions used. This indicates that increasing number of drugs, active ingredients, and drug classes has positive relationship with number of emergency room admissions. It was also noticeable that single active ingredients had the lowest values for this measure followed by single drugs, followed by TC1S1 classes while the highest value were found for single TC1 single classes.

On the other hand, according to the second measure (i.e., Percentage of ER visits referred to as \%ER), it can be observed that the highest proportion of ER visits for a cluster relative to the total of ER visits in the year is found in clusters with 2-4 elements in all categories except for TC1S1 drug class polypharmacy. This may imply that those clusters represent the majority of the polypharmacy detected from patients claims. However, it also reinforces the importance of observing the actual items involved in those polypharmacy. In other words, although using the number of elements as a predictor of problems is one way of looking for polypharmacy impact on ER visits, it is also equally important to investigate and find the patterns of polypharmacy that tend to be associated with ER problems. Supplementary tables of the top 30 single-item, two-item, and three-item polypharmacy categories and their ranking based on ER visits can be found in Appendix $A$.

Finally, our statistical measures include measuring the probability that a patient who belongs to a certain polypharmacy group would visit the ER. This is referred to as PPER1(i) (OR PPER2(i) when we assume the conditional independence). These measures show that patients who have higher number of polypharmacy, independent of the basic type, have higher probability to go to the ER than patients with lower polypharmacy elements. It is important to note here that the results for PPER1 and PPER2 were the same and so we included the values for PPER1 in all Tables and Figures in this section.

\subsubsection{Relating drug polypharmacy to ER}

In this section, we cluster polypharmacy results into groups based on the number of drugs. Then we calculate the three measures we discussed above for each group. Table 4.20 shows the polypharmacy groups and the three measures (i.e., ERPP(i), \% ER, and PPER1(i)).

Figure 4.1 shows the ratio of ER visits to the number of polypharmacy for 5 polypharmacy groups based on the number of drugs. Figure 4.2 shows the proportion of ER visits for given polypharmacy groups based on the number of drugs. Figure $\mathbf{4 . 3}$ shows the probability that a patient in a given polypharmacy group will visit ER in a given year. 
Table 4.20: Three measures relating the number of drugs in polypharmacy groups to ER visits

\begin{tabular}{|c|c|c|c|c|c|c|c|c|c|c|c|}
\hline Group & Year & ER & \#PP & $\begin{array}{c}\text { ERPP = } \\
\text { \#ER/\#PP }\end{array}$ & $\begin{array}{l}\text { Tot } \\
\text { ER }\end{array}$ & \#PT & $\begin{array}{c}\% \\
\text { ER }\end{array}$ & $\begin{array}{l}\text { Tot } \\
\text { \#PT }\end{array}$ & ERP & $\begin{array}{c}\text { Tot } \\
\text { of } \\
\text { ERP }\end{array}$ & PPER \\
\hline$A: 1$ & 011 & 3076 & 13737 & 0.22 & 24250 & 10091 & 0.13 & 38631 & 0.30 & 15.47 & 0.02 \\
\hline B:2-4 & 2011 & 4735 & 44782 & 0.11 & 24250 & 10395 & 0.20 & 38631 & 0.46 & 15.47 & 0.03 \\
\hline$C: 5-8$ & 2011 & 5283 & 67098 & 0.08 & 24250 & 9094 & 0.22 & 38631 & 0.58 & 15.47 & 0.04 \\
\hline $\begin{array}{c}\text { D:9- } \\
12\end{array}$ & 2011 & 5503 & 74485 & 0.07 & 24250 & 8632 & 0.23 & 38631 & 0.64 & 15.47 & 0.04 \\
\hline $\mathrm{E}:>12$ & 2011 & 5653 & 77865 & 0.07 & 24250 & 419 & 0.23 & 38631 & 13.49 & 15.47 & 0.87 \\
\hline$A: 1$ & 012 & 3282 & 14431 & 0.23 & 2782 & 10659 & 0.26 & 27608 & 0.31 & 3.56 & 0.09 \\
\hline B:2-4 & 2012 & 5164 & 17313 & 0.30 & 12782 & 10977 & 0.40 & 27608 & 0.47 & 3.56 & 0.13 \\
\hline C:5-8 & 2012 & 2731 & 6780 & 0.40 & 12782 & 4221 & 0.21 & 27608 & 0.65 & 3.56 & 0.18 \\
\hline $\begin{array}{c}\text { D:9- } \\
12\end{array}$ & 2012 & 1033 & 1939 & 0.53 & 12782 & 1331 & 0.08 & 27608 & 0.78 & 3.56 & 0.22 \\
\hline$E:>12$ & 2012 & 572 & 662 & 0.86 & 2782 & 420 & 0.04 & 27608 & 1.36 & 3.56 & 0.38 \\
\hline$A: 1$ & 2013 & 12133 & 38129 & 0.32 & 13894 & 9643 & 0.23 & 26590 & 0.33 & 3.82 & 0.09 \\
\hline B:2-4 & 2013 & 5266 & 16029 & 0.33 & 13894 & 10311 & 0.38 & 26590 & 0.51 & 3.82 & 0.13 \\
\hline C:5-8 & 2013 & 3028 & 6720 & & 13894 & 4243 & 0.22 & 26590 & 0.71 & 3.82 & 0.19 \\
\hline $\begin{array}{c}\text { D:9- } \\
12\end{array}$ & 2013 & 1725 & 2870 & 0.60 & 13894 & 1909 & 0.12 & 26590 & 0.90 & 3.82 & 0.24 \\
\hline$E:>12$ & 2013 & 658 & 720 & 0.91 & 13894 & 484 & 0.05 & 26590 & 1.36 & 3.82 & 0.36 \\
\hline $\mathrm{A}: 1$ & 2014 & 3239 & 12847 & 0.25 & 14349 & 9598 & 0.23 & 26232 & 0.34 & 4.29 & 0.08 \\
\hline B:2-4 & 2014 & 5433 & 16020 & & 14349 & 10262 & 0.38 & 26232 & 0.53 & 4.29 & 0.12 \\
\hline C:5-8 & 2014 & 3267 & 6958 & & 14349 & 4356 & 0.23 & 26232 & 0.75 & 4.29 & 0.17 \\
\hline $\begin{array}{c}\text { D:9- } \\
12\end{array}$ & 2014 & 1488 & 2131 & 0.70 & 14349 & 1460 & 0.10 & 26232 & 1.02 & 4.29 & 0.24 \\
\hline$E:>12$ & 2014 & 922 & 883 & 1.04 & 14349 & 556 & 0.06 & 26232 & 1.66 & 4.29 & 0.39 \\
\hline$A: 1$ & 2015 & 3030 & 13321 & 0.23 & 14101 & 9886 & 0.21 & 27156 & 0.31 & 4.10 & 0.07 \\
\hline B:2-4 & 2015 & 5347 & 16730 & 0.32 & 14101 & 10644 & 0.38 & 27156 & 0.50 & 4.10 & 0.12 \\
\hline C:5-8 & 2015 & 3265 & 7131 & 0.46 & 14101 & 4474 & 0.23 & 27156 & 0.73 & 4.10 & 0.18 \\
\hline $\begin{array}{c}\text { D:9- } \\
12 \\
\end{array}$ & 2015 & 1542 & 2295 & 0.67 & 14101 & 1572 & 0.11 & 27156 & 0.98 & 4.10 & 0.24 \\
\hline$E:>12$ & 2015 & 917 & 905 & 1.01 & 14101 & 580 & 0.07 & 27156 & 1.58 & 4.10 & 0.39 \\
\hline
\end{tabular}




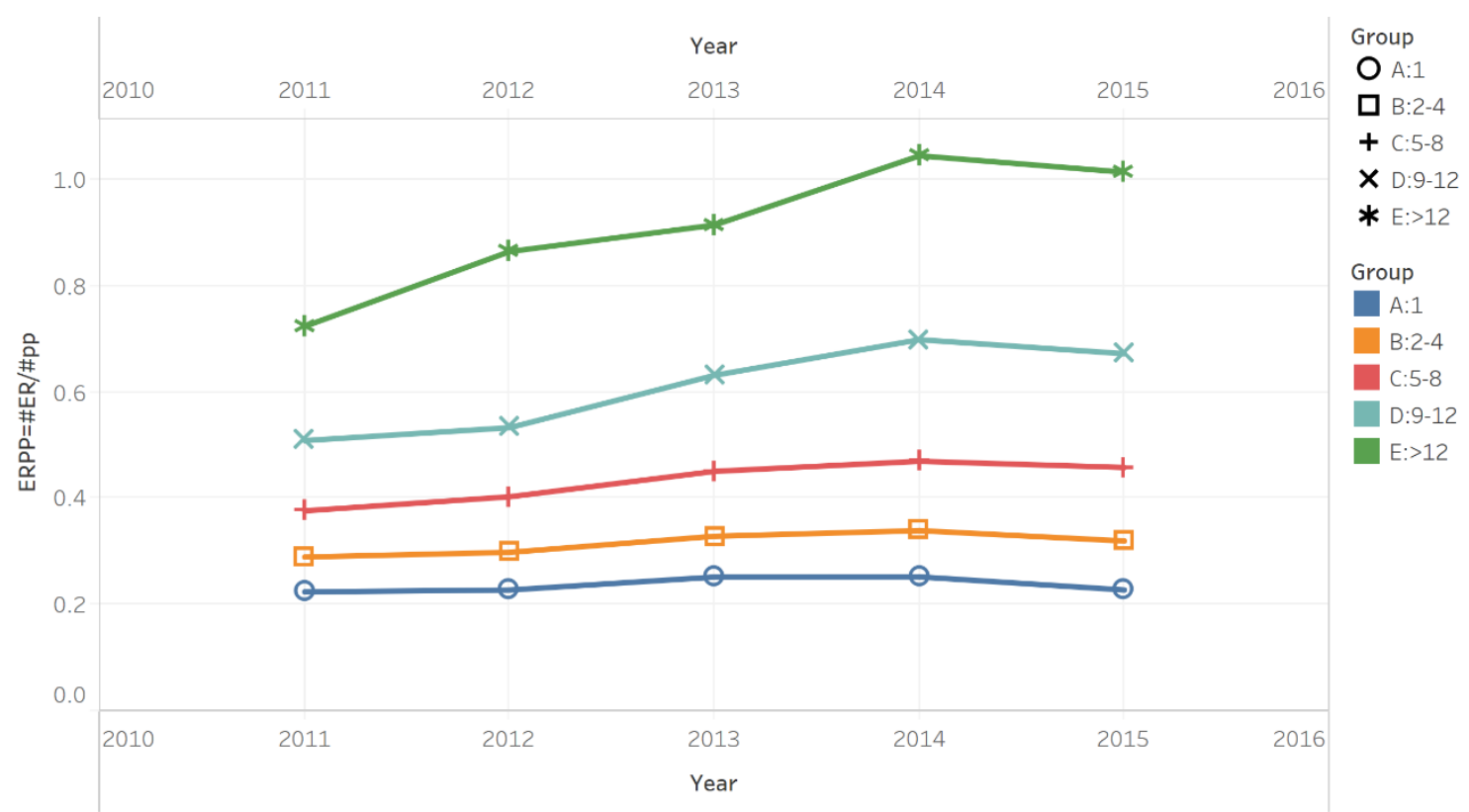

Figure 4.1 Ratio of ER visits to the number of polypharmacy for 5 polypharmacy groups based on the number of drugs.

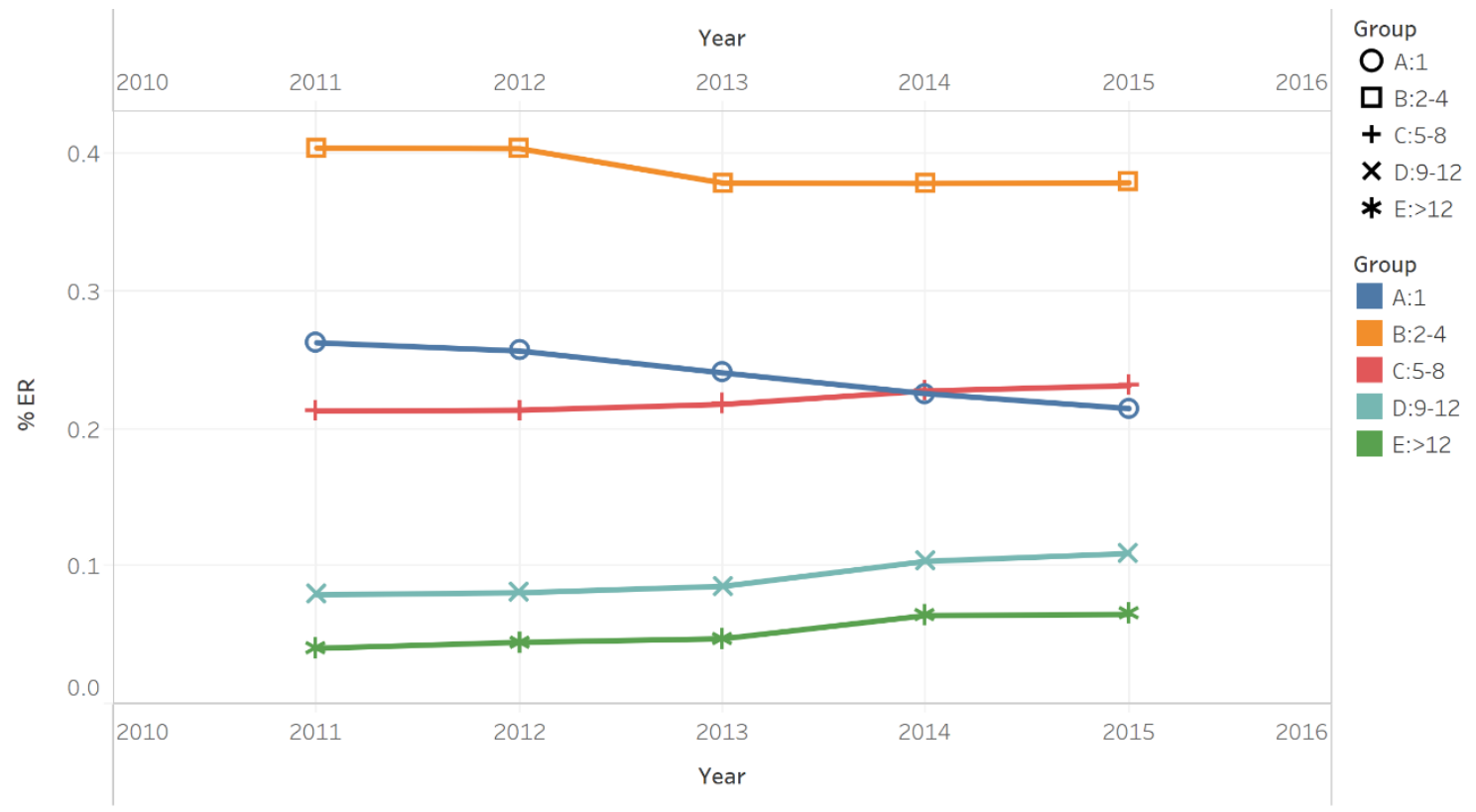

Figure 4.2 Proportion of ER visits for given polypharmacy groups based on the number of drugs. 


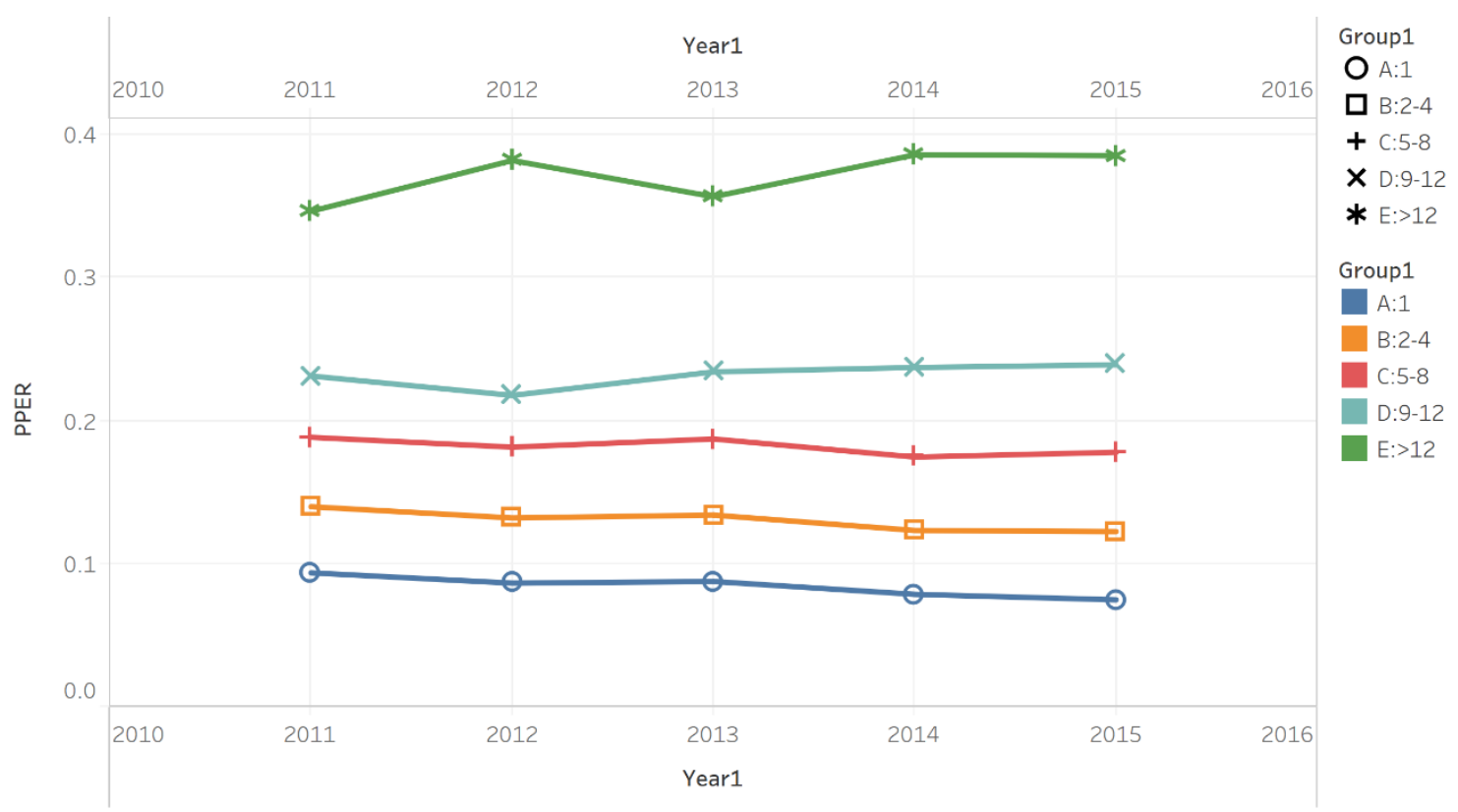

Figure 4.3 The probability that a patient in a given polypharmacy group will visit ER in a given year.

In general, ERPP has been increased with increased number of drugs in all years. This indicates that increasing number of drugs has positive relationship with number of emergency room admissions. It was also noticeable that according to the second measure (i.e., \%ER), it can be observed that the highest proportion of ER visits for a cluster relative to the total of ER visits in the year is found for clusters with 2-4 drugs for all years. This may imply that those clusters represent the majority of the polypharmacy detected from patients claims.

Finally, we calculate the probability that a patient who belongs to a certain polypharmacy group would visit the ER (i.e., PPER1 OR PPER2). These measures show that patients who have higher number of polypharmacy have higher probability to go to the ER than patients with lower polypharmacy elements. In particular, the group with drugs $>12$ has the highest probability value followed by the group with 9-12 drugs followed by the group with 5-8 followed by the group with 2-4 and finally, the group with single drugs. To sum up, the results of the three measures emphasize that the relationship between number of drugs in polypharmacy and the number of emergency room admissions is positive.

\subsubsection{Relating Active Ingredients polypharmacy to ER}

In this section, we cluster polypharmacy results into groups based on the number of active ingredients. Then we calculate the three measures we discussed above (in the introduction of section 4.3) for each group. Table 4.21 shows the polypharmacy groups and the three measures (i.e., ERPP(i), \% ER, and PPER1(i)). Figure 4.4 shows the ratio of ER visits to the number of polypharmacy for 5 polypharmacy groups based on the number of active ingredients. Figure 4.5 shows the proportion of ER visits for given polypharmacy groups based on the number of active 
ingredients. Figure $\mathbf{4 . 6}$ shows the probability that a patient in a given polypharmacy group will visit ER in a given year.

Table 4.21: Three measures relating the number of active ingredients in polypharmacy to ER visits

\begin{tabular}{|c|c|c|c|c|c|c|c|c|c|c|c|}
\hline Group & Year & ER & \#PP & $\begin{array}{c}\text { ERPP }= \\
\text { \#ER/\#PP }\end{array}$ & Tot ER & \#PT & $\%$ ER & $\begin{array}{l}\text { Tot } \\
\text { \#PT }\end{array}$ & ERP & $\begin{array}{l}\text { Tot of } \\
\text { ERP }\end{array}$ & PPER \\
\hline$A: 1$ & 2011 & 2577 & 11964 & 0.22 & 11707 & 8903 & 0.22 & 26500 & 0.29 & 3.08 & 0.09 \\
\hline B:2-4 & 2011 & 4565 & 16572 & 0.28 & 11707 & 10759 & 0.39 & 26500 & 0.42 & 3.08 & 0.14 \\
\hline C:5-8 & 2011 & 2791 & 7453 & 0.37 & 11707 & 4660 & 0.24 & 26500 & 0.60 & 3.08 & 0.19 \\
\hline D:9-12 & 2011 & 1020 & 2183 & 0.47 & 11707 & 1482 & 0.09 & 26500 & 0.69 & 3.08 & 0.22 \\
\hline $\mathrm{E}:>12$ & 2011 & 754 & 1033 & 0.73 & 11707 & 696 & 0.06 & 26500 & 1.08 & 3.08 & 0.35 \\
\hline$A: 1$ & 2012 & 2684 & 12116 & 0.22 & 12144 & 9048 & 0.22 & 26688 & 0.30 & 3.17 & 0.09 \\
\hline B:2-4 & 2012 & 4898 & 16828 & 0.29 & 12144 & 10862 & 0.40 & 26688 & 0.45 & 3.17 & 0.14 \\
\hline C:5-8 & 2012 & 2758 & 7214 & 0.38 & 12144 & 4607 & 0.23 & 26688 & 0.60 & 3.17 & 0.19 \\
\hline D:9-12 & 2012 & 1101 & 2218 & 0.50 & 12144 & 1536 & 0.09 & 26688 & 0.72 & 3.17 & 0.23 \\
\hline$E:>12$ & 2012 & 703 & 940 & 0.75 & 12144 & 635 & 0.06 & 26688 & 1.11 & 3.17 & 0.35 \\
\hline$A: 1$ & 2013 & 2908 & 11798 & 0.25 & 14140 & 9005 & 0.21 & 27733 & 0.32 & 3.59 & 0.09 \\
\hline B:2-4 & 2013 & 5424 & 17095 & 0.32 & 14140 & 11245 & 0.38 & 27733 & 0.48 & 3.59 & 0.13 \\
\hline C:5-8 & 2013 & 3462 & 7818 & 0.44 & 14140 & 5058 & 0.24 & 27733 & 0.68 & 3.59 & 0.19 \\
\hline $\mathrm{D}: 9-12$ & 2013 & 1365 & 2312 & 0.59 & 14140 & 1656 & 0.10 & 27733 & 0.82 & 3.59 & 0.23 \\
\hline $\mathrm{E}:>12$ & 2013 & 981 & 1107 & 0.89 & 14140 & 769 & 0.07 & 27733 & 1.28 & 3.59 & 0.36 \\
\hline$A: 1$ & 2014 & 2731 & 11258 & 0.24 & 14349 & 8496 & 0.19 & 26544 & 0.32 & 3.94 & 0.08 \\
\hline B:2-4 & 2014 & 5326 & 16150 & 0.33 & 14349 & 10552 & 0.37 & 26544 & 0.50 & 3.94 & 0.13 \\
\hline C:5-8 & 2014 & 3468 & 7716 & 0.45 & 14349 & 4949 & 0.24 & 26544 & 0.70 & 3.94 & 0.18 \\
\hline $\mathrm{D}: 9-12$ & 2014 & 1587 & 2450 & 0.65 & 14349 & 1714 & 0.11 & 26544 & 0.93 & 3.94 & 0.24 \\
\hline$E:>12$ & 2014 & 1237 & 1265 & 0.98 & 14349 & 833 & 0.09 & 26544 & 1.48 & 3.94 & 0.38 \\
\hline$A: 1$ & 2015 & 2436 & 11342 & 0.21 & 14101 & 8503 & 0.17 & 27464 & 0.29 & 3.83 & 0.07 \\
\hline B:2-4 & 2015 & 5291 & 17167 & 0.31 & 14101 & 11171 & 0.38 & 27464 & 0.47 & 3.94 & 0.12 \\
\hline C:5-8 & 2015 & 3521 & 8028 & 0.44 & 14101 & 5174 & 0.25 & 27464 & 0.68 & 3.94 & 0.17 \\
\hline $\mathrm{D}: 9-12$ & 2015 & 1698 & 2629 & 0.65 & 14101 & 1826 & 0.12 & 27464 & 0.93 & 3.94 & 0.24 \\
\hline $\mathrm{E}:>12$ & 2015 & 1155 & 1216 & 0.95 & 14101 & 790 & 0.08 & 27464 & 1.46 & 3.94 & 0.37 \\
\hline
\end{tabular}




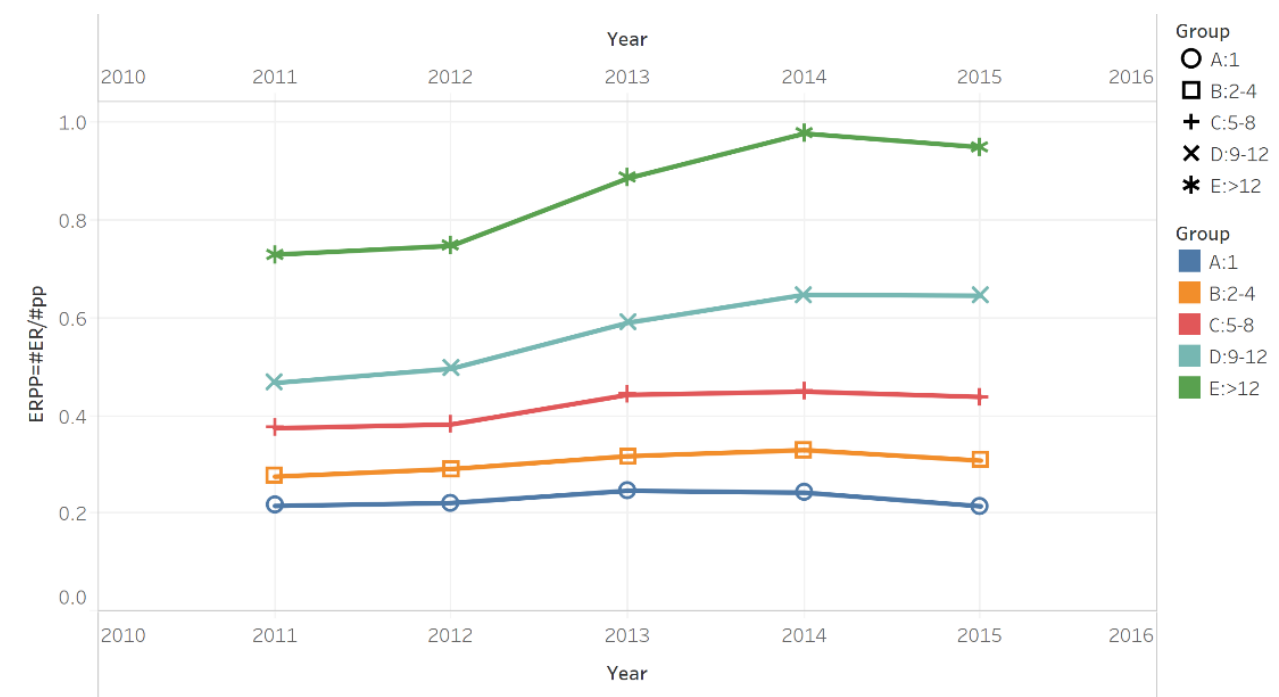

Figure 4.4 Ratio of ER visits to the number of polypharmacy for 5 polypharmacy groups based on the number of active ingredients.

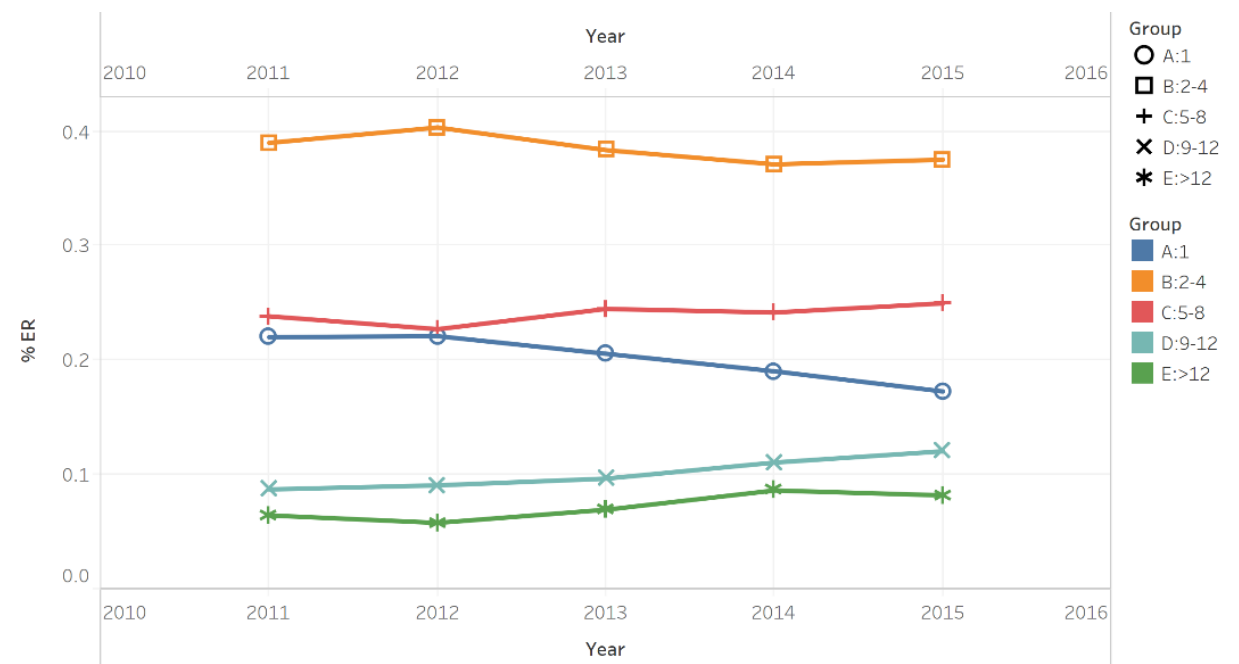

Figure 4.5 Proportion of ER visits for given polypharmacy groups based on the number of active ingredients. 


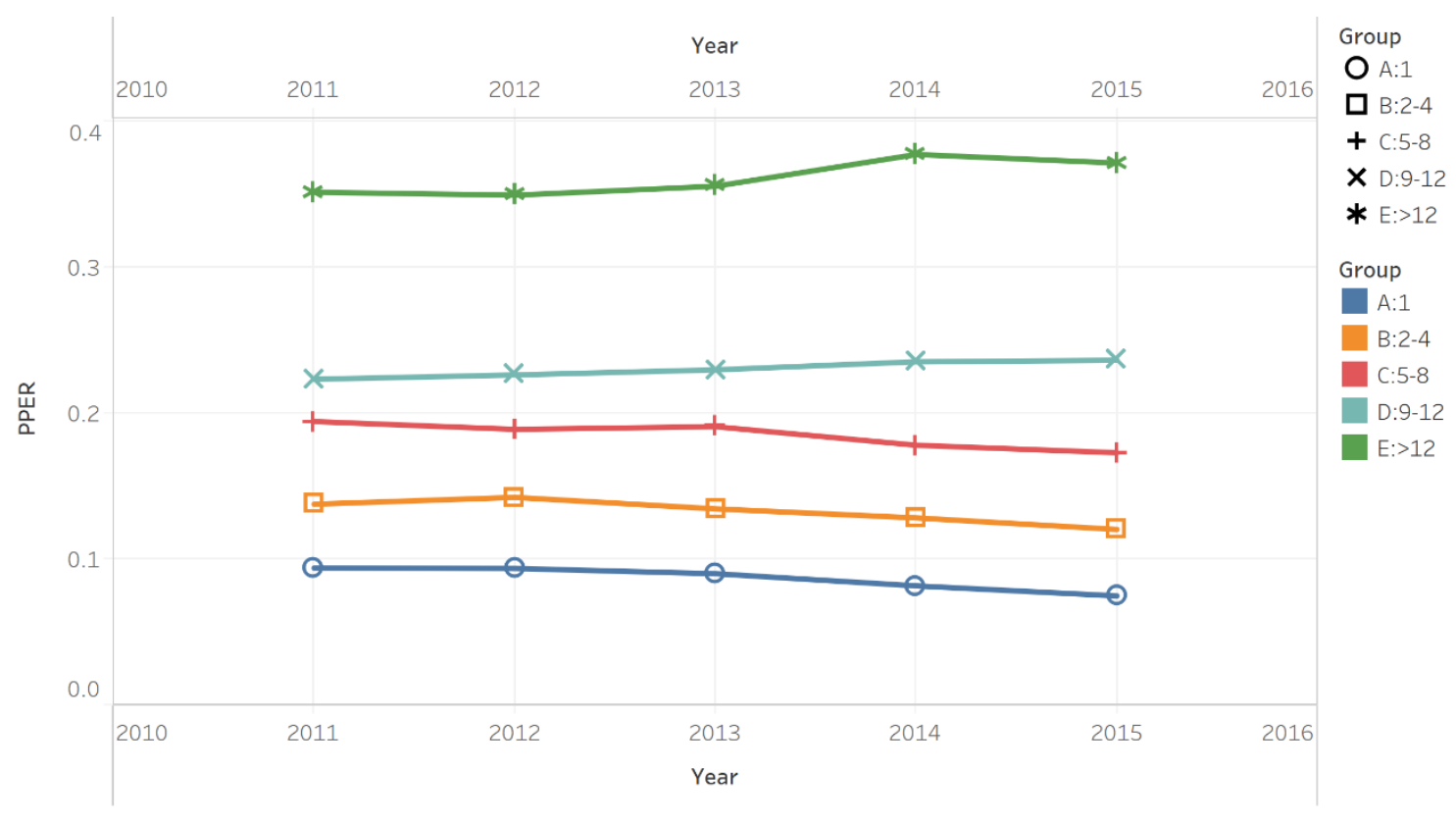

Figure 4.6 The probability that a patient in a given polypharmacy group will visit ER in a given year.

In analysis, ERPP has been increased with increased number of active ingredients in all years. This indicates that increasing number of active ingredients and the number of emergency room admissions are positively correlated. We can observe that based on the second measure (i.e., \%ER), the highest proportion of ER visits for a cluster relative to the total of ER visits in the year is found for clusters with 2-4 active ingredients for all years. This could imply that those clusters represent the biggest group with the highest number of polypharmacy detected from patients claims.

Finally, we calculate the probability that a patient who belongs to a certain polypharmacy group would visit the ER (i.e., PPER1 OR PPER2). These measures show that patients who have higher number of polypharmacy have higher probability to go to the ER than patients with lower polypharmacy elements. In particular, the group with active ingredients $>12$ has the highest probability value followed by the group with 9-12 drugs followed by the group with 5-8 followed by the group with 2-4 and finally, the group with single active ingredients. In conclusion, the results of the three measures emphasize the positive relationship between number of active ingredients in polypharmacy and the number of emergency room admissions.

\subsubsection{Relating polypharmacy based on drug-class to ER}

In this section, we cluster polypharmacy results into groups based on the number of drug classes. We start by TC1 drug class and then we perform the same calculations for TC1S1 drug class. We calculate the three measures we discussed above (in the introduction of section 4.3) for each polypharmacy cluster. Table $\mathbf{4 . 2 2}$ shows the polypharmacy groups and the three 
measures (i.e., ERPP(i), \% ER, and PPER1(i)) for TC1 drug class. Figure 4.7 shows the ratio of ER visits to the number of polypharmacy for 5 polypharmacy groups based on the number of TC1 drug classes. Figure 4.8 shows the proportion of ER visits for given polypharmacy groups based on the number of TC1 drug classes. Figure 4.9 shows the probability that a patient in a given polypharmacy group will visit ER in a given year.

Table 4.22: Three measures relating the number of TC1 classes in polypharmacy to ER visits

\begin{tabular}{|c|c|c|c|c|c|c|c|c|c|c|c|}
\hline Group & Year & ER & \#PP & $\begin{array}{c}\text { ERPP }= \\
\text { \#ER/\#PP }\end{array}$ & Tot ER & \#PT & \% ER & $\begin{array}{l}\text { Tot } \\
\text { \#PT }\end{array}$ & ERP & $\begin{array}{l}\text { Tot of } \\
\text { ERP }\end{array}$ & PPER \\
\hline$A: 1$ & 2011 & 3910 & 16467 & 0.24 & 11707 & 11542 & 0.33 & 24724 & 0.34 & 2.81 & 0.12 \\
\hline$B: 2-4$ & 2011 & 5783 & 18661 & 0.31 & 11707 & 10791 & 0.49 & 24724 & 0.54 & 2.81 & 0.19 \\
\hline C:5-8 & 2011 & 1901 & 3933 & 0.48 & 11707 & 2288 & 0.16 & 24724 & 0.83 & 2.81 & 0.30 \\
\hline $\begin{array}{l}\mathrm{D}: 9- \\
12\end{array}$ & 2011 & 113 & 143 & 0.79 & 11707 & 102 & 0.01 & 24724 & 1.11 & 2.81 & 0.39 \\
\hline$E:>12$ & 2011 & 0 & 1 & 0.00 & 11707 & 1 & 0.00 & 24724 & 0.00 & 2.81 & 0.00 \\
\hline$A: 1$ & 2012 & 4214 & 17399 & 0.24 & 12782 & 12264 & 0.33 & 25989 & 0.34 & 3.24 & 0.11 \\
\hline$B: 2-4$ & 2012 & 6317 & 19543 & 0.32 & 12782 & 11271 & 0.49 & 25989 & 0.56 & 3.24 & 0.17 \\
\hline C:5-8 & 2012 & 2101 & 4024 & 0.52 & 12782 & 2350 & 0.16 & 25989 & 0.89 & 3.24 & 0.28 \\
\hline $\begin{array}{l}\mathrm{D}: 9- \\
12 \\
\end{array}$ & 2012 & 150 & 154 & 0.97 & 12782 & 104 & 0.01 & 25989 & 1.44 & 3.24 & 0.45 \\
\hline $\mathrm{E}:>12$ & 2012 & 0 & 0 & - & 12782 & 0 & 0.00 & 25989 & - & 3.24 & - \\
\hline$A: 1$ & 2013 & 4391 & 16408 & 0.27 & 14140 & 11783 & 0.31 & 25772 & 0.37 & 5.70 & 0.07 \\
\hline B:2-4 & 2013 & 6990 & 19304 & 0.36 & 14140 & 11288 & 0.49 & 25772 & 0.62 & 5.70 & 0.11 \\
\hline C:5-8 & 2013 & 2521 & 4220 & 0.60 & 14140 & 2563 & 0.18 & 25772 & 0.98 & 5.70 & 0.17 \\
\hline $\begin{array}{l}\mathrm{D}: 9- \\
12\end{array}$ & 2013 & 236 & 197 & 1.20 & 14140 & 137 & 0.02 & 25772 & 1.72 & 5.70 & 0.30 \\
\hline$E:>12$ & 2013 & 2 & 1 & 2.00 & 14140 & 1 & 0.00 & 25772 & 2.00 & 5.70 & 0.35 \\
\hline$A: 1$ & 2014 & 4244 & 15546 & 0.27 & 14349 & 11114 & 0.30 & 24679 & 0.38 & 4.11 & 0.09 \\
\hline B:2-4 & 2014 & 6933 & 18584 & 0.37 & 14357 & 10770 & 0.48 & 24679 & 0.64 & 4.11 & 0.16 \\
\hline C:5-8 & 2014 & 2840 & 4467 & 0.64 & 14357 & 2628 & 0.20 & 24679 & 1.08 & 4.11 & 0.26 \\
\hline $\begin{array}{l}\mathrm{D}: 9- \\
12\end{array}$ & 2014 & 332 & 241 & 1.38 & 14357 & 166 & 0.02 & 24679 & 2 & 4.11 & 0.49 \\
\hline $\mathrm{E}:>12$ & 2014 & 0 & 1 & 0 & 14357 & 1 & 0 & 24679 & 0 & 4.11 & 0 \\
\hline$A: 1$ & 2015 & 3880 & 15345 & 0.25 & 13452 & 10928 & 0.30 & 24440 & 0.36 & 3.58 & 0.10 \\
\hline$B: 2-4$ & 2015 & 6403 & 18708 & 0.34 & 13452 & 10764 & 0.48 & 24440 & 0.59 & 3.58 & 0.17 \\
\hline C:5-8 & 2015 & 2931 & 4460 & 0.66 & 13452 & 2588 & 0.20 & 24440 & 1.13 & 3.58 & 0.32 \\
\hline $\begin{array}{l}\mathrm{D}: 9- \\
12\end{array}$ & 2015 & 238 & 229 & 1.04 & 13452 & 159 & 0.02 & 24440 & 1.50 & 3.58 & 0.42 \\
\hline$E:>12$ & 2015 & 0 & 1 & 0.00 & 13452 & 1 & 0.00 & 24440 & 0.00 & 3.58 & 0.00 \\
\hline
\end{tabular}




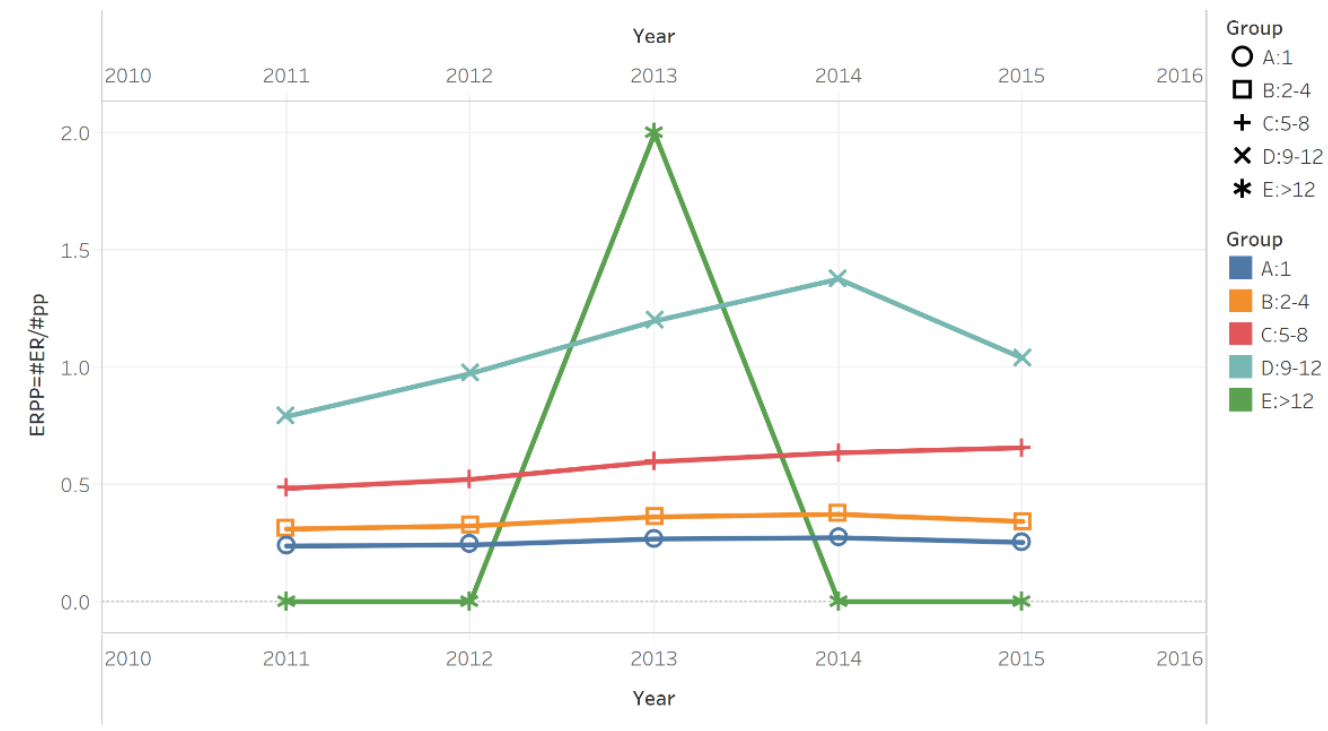

Figure 4.7 Ratio of ER visits to the number of polypharmacy for 5 polypharmacy groups based on the number of TC1 drug class.

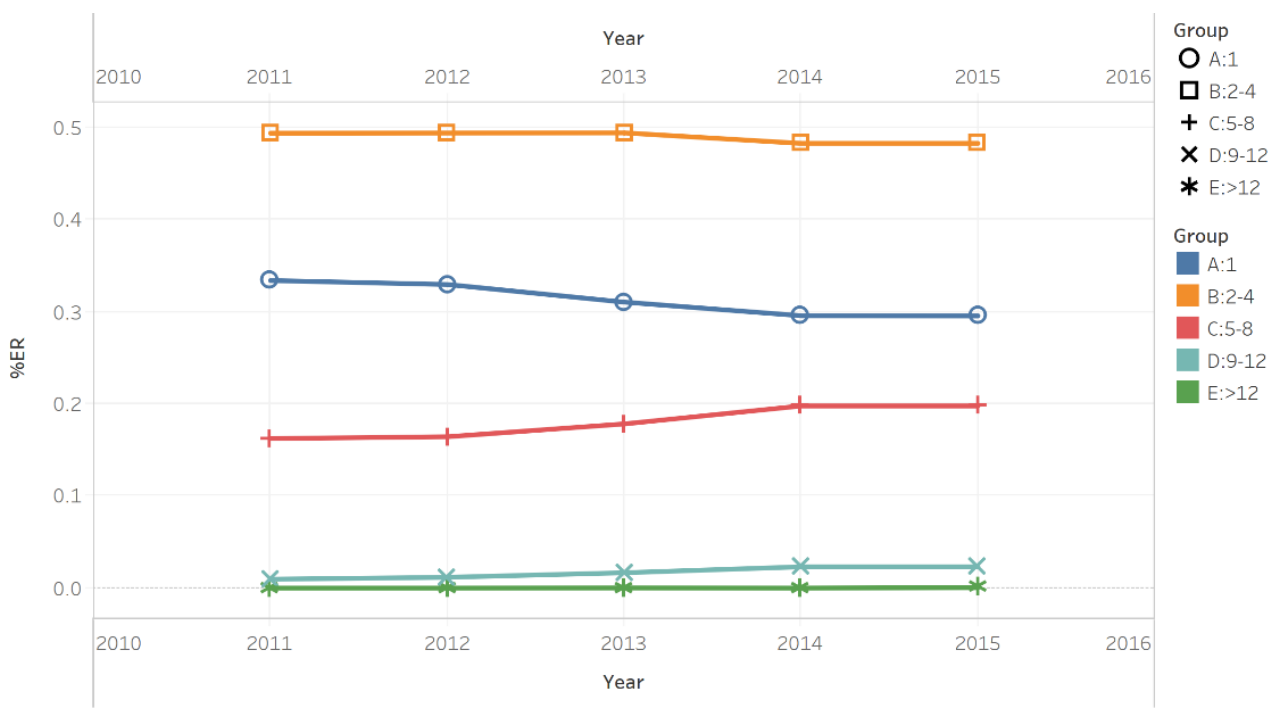

Figure 4.8 Proportion of ER visits for given polypharmacy groups based on the number of TC1 drug class. 


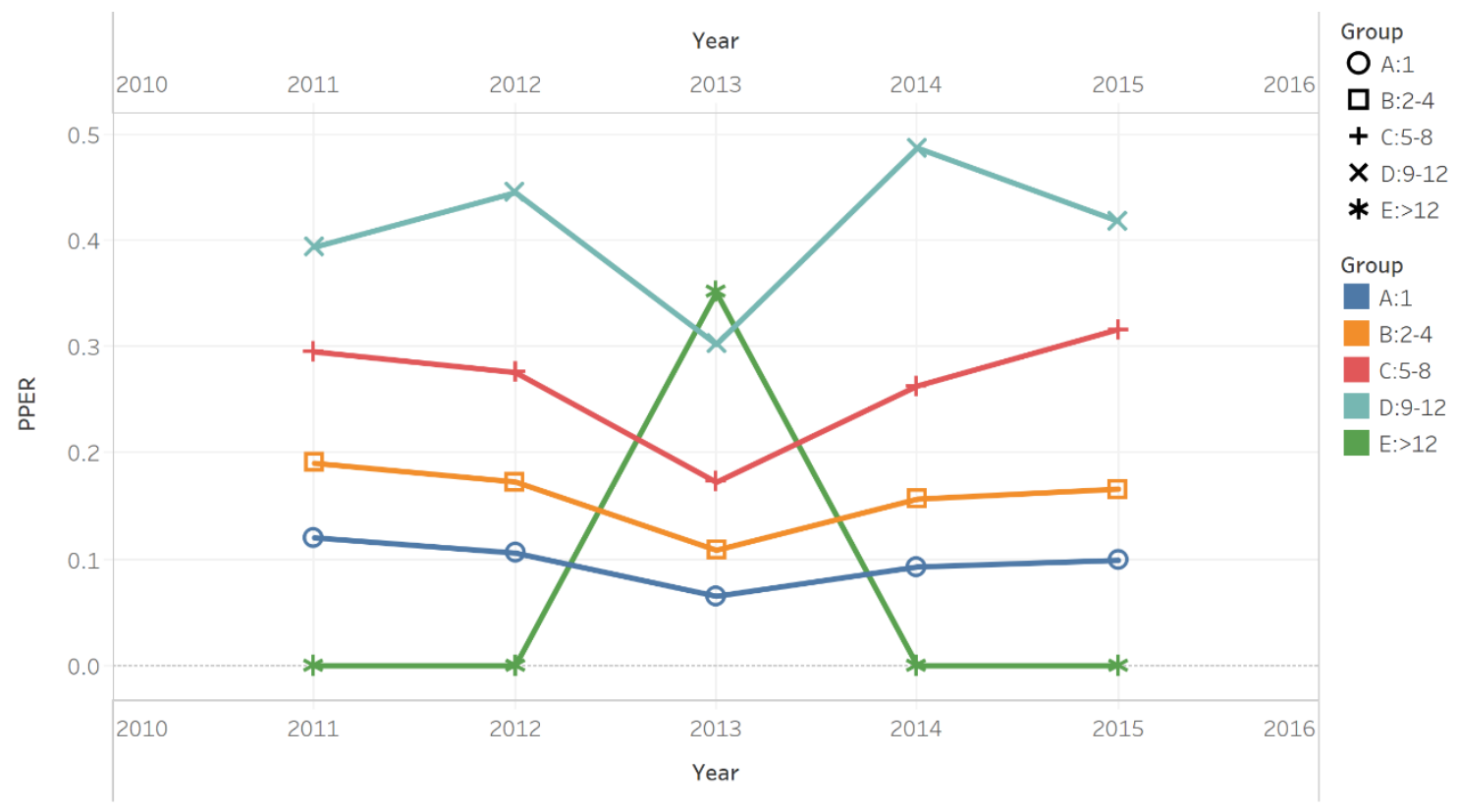

Figure 4.9 The probability that a patient in a given polypharmacy group will visit ER in a given year.

From Table 4.22 and Figure 4.7 we can observe ERPP has been increased with increased number of TC1 drug classes in all years. This indicates that increasing number of TC1 classes has positive relationship with number of emergency room admissions. An exception case was for group E (i.e., which involves polypharmacy with classes greater than 12 ). This group has very low ratio for ERPP. This is explained given that there are much less polypharmacy groups for higher number of elements (i.e., as thresholds for polypharmacy increase, the number of polypharmacy sets found for these thresholds decrease). As a result, no polypharmacy sets were found for thresholds greater than 12 in these years (2011, 2012, 2014, and 2015). However, the remarkable increase for this ratio in 2013 is because this group (E:>12) contains only one patient who has 2 ER visits and one polypharmacy instance with 13 TC1 drug classes. Thus, the ratio \#ER/\#PP value was high (2/1=2) for this case.

On the other hand, we can observe that based on the second measure (i.e., \%ER), the highest proportion of ER visits for a cluster relative to the total of ER visits in the year is found for clusters with 2-4 TC1 drug class for all years. This could imply that those clusters represent the biggest group with the highest number of polypharmacy detected from patients claims. This group is followed by group A: single TC1 drug classes, followed by group C: $5-8$, followed by group D:9-12, and finally group $E:>12$ which has the lowest proportion of ER visits relative to the total ER visits.

Finally, we calculate the probability that a patient who belongs to a certain polypharmacy group would visit the ER (i.e., PPER1 OR PPER2). These measures show that patients who have higher number of polypharmacy have higher probability to go to the ER than patients with lower polypharmacy elements. This was true starting from group D: 9-12 to group A: single TC1 drug classes. The group $\mathrm{E}$ with higher than 12 classes has very low probability due to the poor number 
of polypharmacy in this group. The probability for this group has only increased in 2013 as the \#ER visits was 2 and the number of the patients was 1 , which makes the ratio $\operatorname{ERP}(\mathrm{i})=\frac{\# E R(i)}{\# P T(i)}$ relatively high. To sum up, the results of the three measures emphasize the positive relationship between number of TC1 drug classes in polypharmacy and the number of emergency room admissions. As for TC1S1 drug class, Table $\mathbf{4 . 2 3}$ shows the polypharmacy groups and the three measures (i.e., ERPP(i), \% ER, and PPER1(i)) for TC1S1 drug class. Figure $\mathbf{4 . 1 0}$ shows the ratio of ER visits to the number of polypharmacy for 5 polypharmacy groups based on the number of TC1S1 drug classes. Figure $\mathbf{4 . 1 1}$ shows the proportion of ER visits for given polypharmacy groups based on the number of TC1S1 drug classes. Figure $\mathbf{4 . 1 2}$ shows the probability that a patient in a given polypharmacy group will visit ER in a given year.

Table 4.23: Three measures relating the number of TC1S1 classes in polypharmacy to ER visits

\begin{tabular}{|c|c|c|c|c|c|c|c|c|c|c|c|}
\hline Group & Year & ER & \#PP & $\begin{array}{c}\text { ERPP = } \\
\text { \#ER/\#PP }\end{array}$ & Tot ER & \#PT & \% ER & $\begin{array}{l}\text { Tot } \\
\text { \#PT }\end{array}$ & ERP & $\begin{array}{l}\text { Tot of } \\
\text { ERP }\end{array}$ & PPER \\
\hline$A: 1$ & 2011 & 3347 & 14641 & 0.23 & 11707 & 10565 & 0.29 & 25639 & 0.32 & 3.51 & 0.09 \\
\hline$B: 2-4$ & 2011 & 5043 & 16939 & 0.30 & 11707 & 10457 & 0.43 & 25639 & 0.48 & 3.51 & 0.14 \\
\hline$C: 5-8$ & 2011 & 2329 & 6048 & 0.39 & 11707 & 3581 & 0.20 & 25639 & 0.65 & 3.51 & 0.19 \\
\hline D:9-12 & 2011 & 801 & 1346 & 0.60 & 11707 & 873 & 0.07 & 25639 & 0.92 & 3.51 & 0.26 \\
\hline$E:>12$ & 2011 & 187 & 231 & 0.81 & 11707 & 163 & 0.02 & 25639 & 1.15 & 3.51 & 0.33 \\
\hline$A: 1$ & 2012 & 3620 & 15441 & 0.23 & 12782 & 11219 & 0.28 & 26982 & 0.32 & 3.92 & 0.08 \\
\hline$B: 2-4$ & 2012 & 5424 & 17767 & 0.31 & 12782 & 10931 & 0.42 & 26982 & 0.50 & 3.92 & 0.13 \\
\hline C:5-8 & 2012 & 2672 & 6322 & 0.42 & 12782 & 3777 & 0.21 & 26982 & 0.71 & 3.92 & 0.18 \\
\hline D:9-12 & 2012 & 818 & 1336 & 0.61 & 12782 & 886 & 0.06 & 26982 & 0.92 & 3.92 & 0.24 \\
\hline$E:>12$ & 2012 & 248 & 254 & 0.98 & 12782 & 169 & 0.02 & 26982 & 1.47 & 3.92 & 0.37 \\
\hline$A: 1$ & 2013 & 3732 & 14451 & 0.26 & 14140 & 10725 & 0.26 & 26756 & 0.35 & 4.37 & 0.08 \\
\hline B:2-4 & 2013 & 5802 & 17348 & 0.33 & 14140 & 10826 & 0.41 & 26756 & 0.54 & 4.37 & 0.12 \\
\hline$C: 5-8$ & 2013 & 3206 & 6588 & 0.49 & 14140 & 3999 & 0.23 & 26756 & 0.80 & 4.37 & 0.18 \\
\hline D:9-12 & 2013 & 1073 & 1450 & 0.74 & 14140 & 1003 & 0.08 & 26756 & 1.07 & 4.37 & 0.25 \\
\hline$E:>12$ & 2013 & 327 & 293 & 1.12 & 14140 & 203 & 0.02 & 26756 & 1.61 & 4.37 & 0.37 \\
\hline$A: 1$ & 2014 & 3618 & 13778 & 0.26 & 14532 & 10133 & 0.25 & 25760 & 0.36 & 4.70 & 0.08 \\
\hline$B: 2-4$ & 2014 & 5667 & 16497 & 0.34 & 14532 & 10248 & 0.39 & 25760 & 0.55 & 4.70 & 0.12 \\
\hline$C: 5-8$ & 2014 & 3457 & 6655 & 0.52 & 14532 & 4012 & 0.24 & 25760 & 0.86 & 4.70 & 0.18 \\
\hline D:9-12 & 2014 & 1171 & 1551 & 0.75 & 14532 & 1020 & 0.08 & 25760 & 1.15 & 4.70 & 0.24 \\
\hline$E:>12$ & 2014 & 619 & 550 & 1.13 & 14532 & 347 & 0.04 & 25760 & 1.78 & 4.70 & 0.38 \\
\hline$A: 1$ & 2015 & 3359 & 14133 & 0.24 & 14101 & 10355 & 0.24 & 26498 & 0.32 & 4.53 & 0.07 \\
\hline$B: 2-4$ & 2015 & 5695 & 17414 & 0.33 & 14101 & 10746 & 0.40 & 26498 & 0.53 & 4.53 & 0.12 \\
\hline$C: 5-8$ & 2015 & 3347 & 6802 & 0.49 & 14101 & 4056 & 0.24 & 26498 & 0.83 & 4.53 & 0.18 \\
\hline D:9-12 & 2015 & 1295 & 1669 & 0.78 & 14101 & 1099 & 0.09 & 26498 & 1.18 & 4.53 & 0.26 \\
\hline$E:>12$ & 2015 & 405 & 364 & 1.11 & 14101 & 242 & 0.03 & 26498 & 1.67 & 4.53 & 0.37 \\
\hline
\end{tabular}




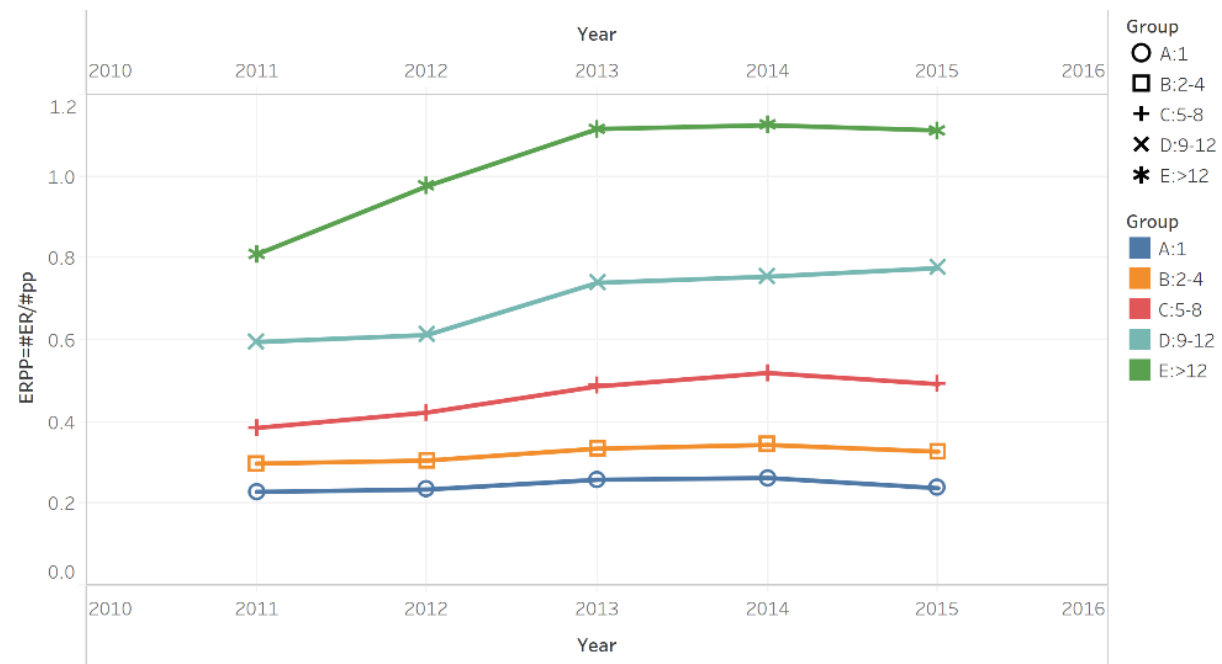

Figure 4.10 Ratio of ER visits to the number of polypharmacy for 5 polypharmacy groups based on the number of TC1S1 drug class.

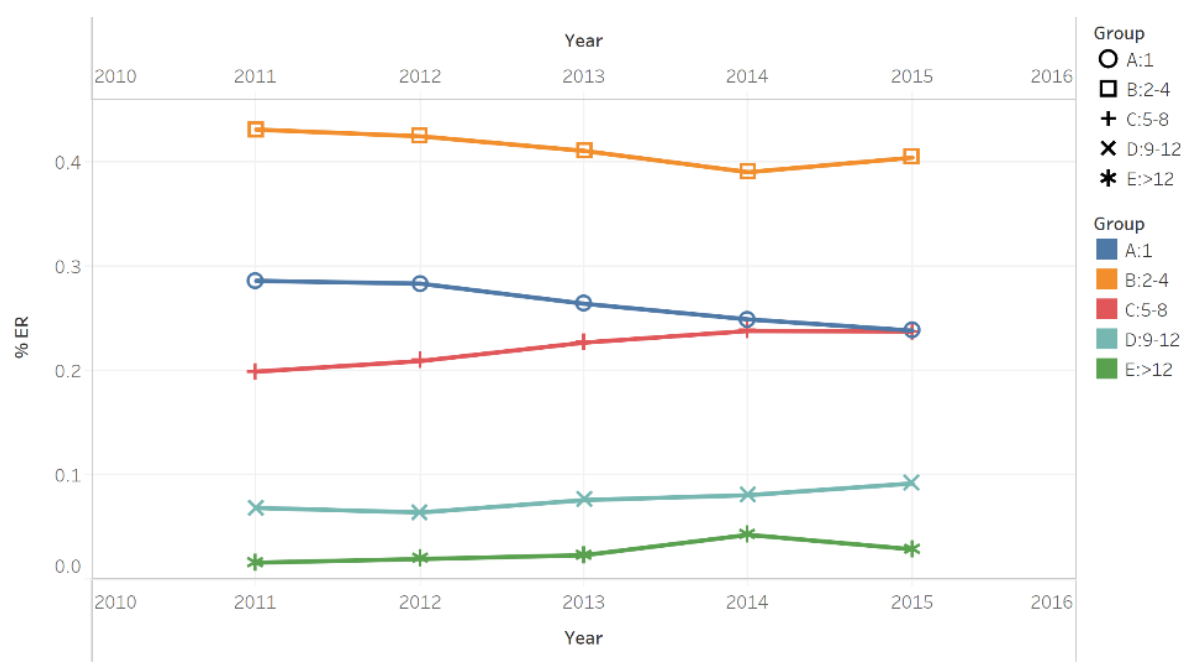

Figure 4.11 Proportion of ER visits for given polypharmacy groups based on the number of TC1S1 drug class. 


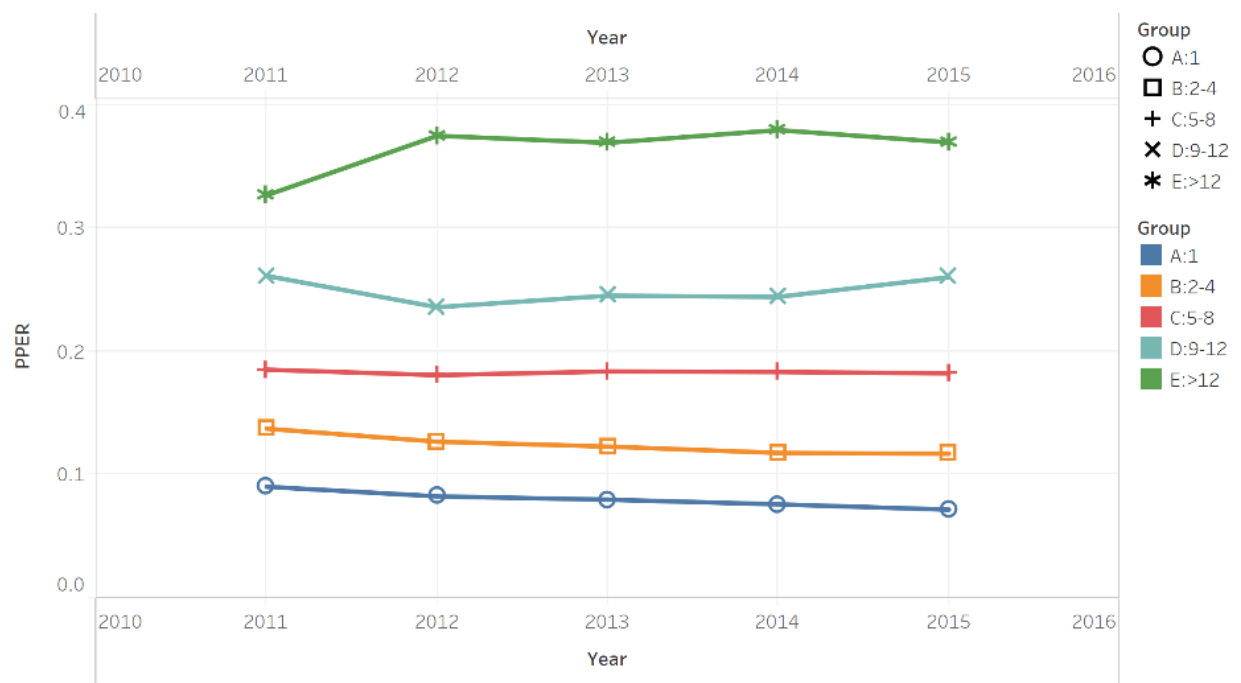

Figure 4.12 The probability that a patient in a given polypharmacy group will visit ER in a given year.

In analysis, ERPP has been increased with increased number of active ingredients in all years. This indicates that increasing number of TC1S1 drug class elements and the number of emergency room admissions are positively correlated. We can observe that based on the second measure (i.e., \%ER), the highest proportion of ER visits for a cluster relative to the total of ER visits in the year is found for clusters with 2-4 TC1S1 drug classes for all years. This means that those clusters have highest number of polypharmacy detected from patients claims. After that, we calculate the probability that a patient who belongs to a certain polypharmacy group would visit the ER (i.e., PPER1 OR PPER2). These measures show that patients who have higher number of polypharmacy have higher probability to go to the ER than patients with lower polypharmacy elements. Thus, the group with TC1S1 drug classes $>12$ has the highest probability value followed by the group with 9-12 drugs followed by the group with 5-8 followed by the group with 2-4 and finally, the group with single drug classes of this type. In conclusion, the results of the three measures emphasize the positive relationship between number of TC1S1 drug classes in polypharmacy and the number of ER visits.

Finally, we combine the results for all years for every definition. Table 4.24 shows a summary of average values for all definitions and for all years.

Table 4.24: Summary of average values for all definitions and for all years.

\begin{tabular}{|l|c|c|c|c|c|c|c|l|l|l|l|}
\hline Type & Group & ER & \#PP & $\begin{array}{l}\text { ERPP= } \\
\text { \#ER/ } \\
\text { \#pp }\end{array}$ & Tot ER & \#PT & \% ER & $\begin{array}{l}\text { Tot } \\
\text { \#PT }\end{array}$ & ERP & $\begin{array}{l}\text { Tot } \\
\text { of } \\
\text { ERP }\end{array}$ & \begin{tabular}{l} 
PPER \\
\hline drug
\end{tabular} \\
\hline A:1 & 3168.8 & 13423 & 0.24 & 13259.2 & 8903 & 0.24 & 26500 & 0.36 & 3.32 & 0.11 \\
\hline drug & B:2-4 & 5189 & 16490.4 & 0.31 & 13259.2 & 10759 & 0.39 & 26500 & 0.48 & 3.32 & 0.15 \\
\hline drug & C:5-8 & 2957 & 6843.2 & 0.43 & 13259.2 & 4660 & 0.22 & 26500 & 0.63 & 3.32 & 0.19 \\
\hline drug & D:9-12 & 1236 & 2014.8 & 0.61 & 13259.2 & 1482 & 0.09 & 26500 & 0.83 & 3.32 & 0.25 \\
\hline drug & E:>12 & 708.4 & 764.8 & 0.93 & 13259.2 & 696 & 0.05 & 26500 & 1.02 & 3.32 & 0.31 \\
\hline
\end{tabular}




\begin{tabular}{|c|c|c|c|c|c|c|c|c|c|c|c|}
\hline & & & & & & & & & & & \\
\hline $\mathrm{Al}$ & $\mathrm{A}: 1$ & 2667.2 & 11695.6 & 0.23 & 13288.2 & 8903 & 0.20 & 26500 & 0.30 & 3.76 & 0.08 \\
\hline $\mathrm{Al}$ & $\mathrm{B}: 2-4$ & 5100.8 & 16762.4 & 0.30 & 13288.2 & 10759 & 0.38 & 26500 & 0.47 & 3.76 & 0.13 \\
\hline $\mathrm{Al}$ & $\mathrm{C}: 5-8$ & 3200 & 7645.8 & 0.42 & 13288.2 & 4660 & 0.24 & 26500 & 0.69 & 3.76 & 0.18 \\
\hline $\mathrm{Al}$ & $\mathrm{D}: 9-12$ & 1354.2 & 2358.4 & 0.57 & 13288.2 & 1482 & 0.10 & 26500 & 0.91 & 3.76 & 0.24 \\
\hline $\mathrm{Al}$ & $\mathrm{E}:>12$ & 966 & 1112.2 & 0.87 & 13288.2 & 696 & 0.07 & 26500 & 1.39 & 3.76 & 0.37 \\
\hline & & & & & & & & & & & \\
\hline TC1 & $\mathrm{A}: 1$ & 4127.8 & 16233 & 0.25 & 13286 & 8903 & 0.31 & 26500 & 0.46 & 1.74 & 0.27 \\
\hline TC1 & $\mathrm{B}: 2-4$ & 6485.2 & 18960 & 0.34 & 13286 & 10759 & 0.49 & 26500 & 0.60 & 1.74 & 0.35 \\
\hline TC1 & $\mathrm{C}: 5-8$ & 2458.8 & 4220.8 & 0.58 & 13286 & 4660 & 0.19 & 26500 & 0.53 & 1.74 & 0.30 \\
\hline TC1 & $\mathrm{D}: 9-12$ & 213.8 & 192.8 & 1.11 & 13286 & 1482 & 0.02 & 26500 & 0.14 & 1.74 & 0.08 \\
\hline TC1 & $\mathrm{E}:>12$ & 0.4 & 0.8 & 0.50 & 13286 & 696 & 0.00 & 26500 & 0.00 & 1.74 & 0.00 \\
\hline & & & & & & & & & & & \\
\hline TC1S1 & $\mathrm{A}: 1$ & 3535.2 & 14488.8 & 0.24 & 13452.4 & 8903 & 0.26 & 26500 & 0.40 & 2.76 & 0.14 \\
\hline TC1S1 & $\mathrm{B}: 2-4$ & 5526.2 & 17193 & 0.32 & 13452.4 & 10759 & 0.41 & 26500 & 0.51 & 2.76 & 0.19 \\
\hline TC1S1 & $\mathrm{C}: 5-8$ & 3002.2 & 6483 & 0.46 & 13452.4 & 4660 & 0.22 & 26500 & 0.64 & 2.76 & 0.23 \\
\hline TC1S1 & $\mathrm{D}: 9-12$ & 1031.6 & 1470.4 & 0.70 & 13452.4 & 1482 & 0.08 & 26500 & 0.70 & 2.76 & 0.25 \\
\hline TC1S1 & $\mathrm{E}:>12$ & 357.2 & 338.4 & 1.06 & 13452.4 & 696 & 0.03 & 26500 & 0.51 & 2.76 & 0.19 \\
\hline
\end{tabular}

We can observe from Table 4.24 that single active ingredients had the lowest values for ERPP followed by single drugs, followed by single TC1S1 classes while the highest value were found for single TC1 single classes. This is naturally as drugs with single active ingredients should be associated with less risk and drug classes that contains more elements are more likely to be associated with higher risk.

\subsection{Richness and Over-Representation}

As discussed in Chapter 3, the richness of a polypharmacy is used to measure the proportion of the actual observation to the expected observation. We study richness using two points of view: richness of occurrence and richness of occurrence with ER. One point to consider here is that richness could not be calculated for combinations of drug/active ingredients/drug classes if the occurrences of their single components could not be found. i.e., richness of AMOXICILLIN--IBUPROFEN cannot be calculated if the occurrence of AMOXICILLIN or IBUPROFEN as single elements was not reported.

The next sub-sections contain multiple tables with richness values for the top 30 twoelement polypharmacy sets ranked based on ER visits. We will identify an exemplary case where the richness defined for the co-occurrence of two items (i.e., two drugs, two active ingredients, or two drug classes) have noticeable lower values in comparison to the richness of ER. Tables for richness for the top 30 three-element polypharmacy can be found in Appendix B.

\subsubsection{Richness of polypharmacy using number of drugs}

This section contains tables with richness values for the top 30 two-drug polypharmacy sets ranked based on ER visits. Table $\mathbf{4 . 2 5}$ shows the top drug-pairs and their co-occurrences 
probability ranked by ER total and Table 4.26 shows the individual and joint probability of occurrence of the top 30 two-drug polypharmacy (sorted by ER total) with richness of occurrence and ER.

Table 4.25: Top 30 drug-pairs and their co-occurrences probability ranked by ER total.

\begin{tabular}{|c|c|c|c|c|c|}
\hline & Two-drug polypharmacy & Occ & ER & \#ER/\#OCc & $\mathrm{p}(\mathrm{two}-\mathrm{occ})^{* *}$ \\
\hline 1 & HYDROCO/APAP--IBUPROFEN & 176 & 147 & 0.84 & 0.0045 \\
\hline 2 & AMOXICILLIN--IBUPROFEN & 294 & 94 & 0.32 & 0.0074 \\
\hline 3 & AMOXICILLIN--HYDROCO/APAP & 157 & 64 & 0.41 & 0.0040 \\
\hline 4 & APAP/CODEINE--AZITHROMYCIN & 116 & 56 & 0.48 & 0.0029 \\
\hline 5 & ALBUTEROL--AMOXICILLIN & 84 & 56 & 0.67 & 0.0021 \\
\hline 6 & AMOXICILLIN--AZITHROMYCIN & 96 & 54 & 0.56 & 0.0024 \\
\hline 7 & AZITHROMYCIN--HYDROCO/APAP & 83 & 53 & 0.64 & 0.0021 \\
\hline 8 & CYCLOBENZAPR--HYDROCO/APAP & 77 & 52 & 0.68 & 0.0020 \\
\hline 9 & CYCLOBENZAPR--IBUPROFEN & 82 & 50 & 0.61 & 0.0021 \\
\hline 10 & IBUPROFEN--OXYCOD/APAP & 67 & 47 & 0.70 & 0.0017 \\
\hline 11 & IBUPROFEN--PREDNISONE & 14 & 46 & 3.29 & 0.0004 \\
\hline 12 & AMLODIPINE--LISINOPRIL & 143 & 43 & 0.30 & 0.0036 \\
\hline 13 & AZITHROMYCIN--IBUPROFEN & 120 & 43 & 0.36 & 0.0030 \\
\hline 14 & CLINDAMYCIN--HYDROCO/APAP & 41 & 43 & 1.05 & 0.0010 \\
\hline 15 & AZITHROMYCIN--PREDNISONE & 63 & 36 & 0.57 & 0.0016 \\
\hline 16 & APAP/CODEINE--IBUPROFEN & 48 & 36 & 0.75 & 0.0012 \\
\hline 17 & $\begin{array}{l}\text { HYDROCO/APAP-- } \\
\text { PROMETHAZINE }\end{array}$ & 23 & 30 & 1.30 & 0.0006 \\
\hline 18 & LISINOPRIL--SIMVASTATIN & 221 & 29 & 0.13 & 0.0056 \\
\hline 19 & IBUPROFEN--TRAMADOL HCL & 26 & 29 & 1.12 & 0.0007 \\
\hline 20 & ALBUTEROL--PREDNISONE & 32 & 28 & 0.88 & 0.0008 \\
\hline 21 & CYCLOBENZAPR--NAPROXEN & 66 & 27 & 0.41 & 0.0017 \\
\hline 22 & ALBUTEROL--AZITHROMYCIN & 59 & 26 & 0.44 & 0.0015 \\
\hline 23 & HYDROCO/APAP--PENICILLN VK & 53 & 26 & 0.49 & 0.0013 \\
\hline 24 & AMOXICILLIN--PREDNISONE & 48 & 26 & 0.54 & 0.0012 \\
\hline 25 & AMLODIPINE--HYDROCHLOROT & 96 & 23 & 0.24 & 0.0024 \\
\hline 26 & CEPHALEXIN--HYDROCO/APAP & 51 & 23 & 0.45 & 0.0013 \\
\hline 27 & AMOXICILLIN--APAP/CODEINE & 63 & 22 & 0.35 & 0.0016 \\
\hline 28 & SERTRALINE--TRAZODONE & 25 & 21 & 0.84 & 0.0006 \\
\hline 29 & HYDROCO/APAP--NAPROXEN & 37 & 20 & 0.54 & 0.0009 \\
\hline 30 & HYDROCO/APAP--OXYCOD/APAP & 25 & 20 & 0.80 & 0.0006 \\
\hline
\end{tabular}

${ }^{* *} \mathrm{p}$ (two-occ) here represents the joint probability for the pairs. 
Table 4.26: Individual and joint probability of occurrence for the top 30 two-drug polypharmacy (sorted by ER total) with richness of occurrence and ER.

\begin{tabular}{|c|c|c|c|c|c|c|c|c|c|c|c|c|c|}
\hline & part1 & part2 & $\begin{array}{l}\text { part1- } \\
\text { occ }\end{array}$ & $\begin{array}{l}\text { part2- } \\
\text { occ }\end{array}$ & $\begin{array}{c}\text { p(part1- } \\
\text { occ) }\end{array}$ & $\begin{array}{l}\text { p(part2- } \\
\text { occ) }\end{array}$ & $\begin{array}{l}\text { Occ- } \\
\text { richness }\end{array}$ & $\begin{array}{c}\text { part1- } \\
\text { ER } \\
\text { Occ }\end{array}$ & $\begin{array}{c}\text { part2- } \\
\text { ER } \\
\text { Occ }\end{array}$ & $\begin{array}{l}\text { p(part1- } \\
\text { ERocc) }\end{array}$ & $\begin{array}{c}\text { p(part2- } \\
\text { ERocc) }\end{array}$ & $\begin{array}{c}\text { p( } \\
\text { two- } \\
\text { case- } \\
\text { ER) }\end{array}$ & $\begin{array}{c}\text { ER- } \\
\text { richness }\end{array}$ \\
\hline 1 & HYDROCO/APAP & IBUPROFEN & 1238 & 1675 & 0.0182 & 0.0247 & 9.90 & 698 & 691 & 0.044 & 0.043 & 0.013 & 6.756 \\
\hline 2 & AMOXICILLIN & IBUPROFEN & 4471 & 1675 & 0.0659 & 0.0247 & 4.58 & 1190 & 691 & 0.074 & 0.043 & 0.008 & 2.534 \\
\hline 3 & AMOXICILLIN & HYDROCO/APAP & 4471 & 1238 & 0.0659 & 0.0182 & 3.31 & 1190 & 698 & 0.074 & 0.044 & 0.006 & 1.708 \\
\hline 4 & APAP/CODEINE & AZITHROMYCIN & 614 & 3180 & 0.0090 & 0.0469 & 6.93 & 304 & 891 & 0.019 & 0.056 & 0.005 & 4.583 \\
\hline 5 & ALBUTEROL & AMOXICILLIN & 785 & 4471 & 0.0116 & 0.0659 & 2.79 & 275 & 1190 & 0.017 & 0.074 & 0.005 & 3.793 \\
\hline 6 & AMOXICILLIN & AZITHROMYCIN & 4471 & 3180 & 0.0659 & 0.0469 & 0.79 & 1190 & 891 & 0.074 & 0.056 & 0.005 & 1.129 \\
\hline 7 & AZITHROMYCIN & HYDROCO/APAP & 3180 & 1238 & 0.0469 & 0.0182 & 2.46 & 891 & 698 & 0.056 & 0.044 & 0.005 & 1.889 \\
\hline 8 & CYCLOBENZAPR & HYDROCO/APAP & 260 & 1238 & 0.0038 & 0.0182 & 27.90 & 122 & 698 & 0.008 & 0.044 & 0.004 & 13.536 \\
\hline 9 & CYCLOBENZAPR & IBUPROFEN & 260 & 1675 & 0.0038 & 0.0247 & 21.96 & 122 & 691 & 0.008 & 0.043 & 0.004 & 13.148 \\
\hline 10 & IBUPROFEN & OXYCOD/APAP & 1675 & 324 & 0.0247 & 0.0048 & 14.40 & 691 & 175 & 0.043 & 0.011 & 0.004 & 8.616 \\
\hline 11 & IBUPROFEN & PREDNISONE & 1675 & 436 & 0.0247 & 0.0064 & 2.24 & 691 & 190 & 0.043 & 0.012 & 0.004 & 7.767 \\
\hline 12 & AMLODIPINE & LISINOPRIL & 684 & 1737 & 0.0101 & 0.0256 & 14.04 & 81 & 291 & 0.005 & 0.018 & 0.004 & 40.440 \\
\hline 13 & AZITHROMYCIN & IBUPROFEN & 3180 & 1675 & 0.0469 & 0.0247 & 2.63 & 891 & 691 & 0.056 & 0.043 & 0.004 & 1.548 \\
\hline 14 & CLINDAMYCIN & HYDROCO/APAP & 260 & 1238 & 0.0038 & 0.0182 & 14.85 & 77 & 698 & 0.005 & 0.044 & 0.004 & 17.735 \\
\hline 15 & AZITHROMYCIN & PREDNISONE & 3180 & 436 & 0.0469 & 0.0064 & 5.30 & 891 & 190 & 0.056 & 0.012 & 0.003 & 4.714 \\
\hline 16 & APAP/CODEINE & IBUPROFEN & 614 & 1675 & 0.0090 & 0.0247 & 5.44 & 304 & 691 & 0.019 & 0.043 & 0.003 & 3.799 \\
\hline 17 & HYDROCO/APAP & PROMETHAZINE & 1238 & 187 & 0.0182 & 0.0028 & 11.59 & 698 & 85 & 0.044 & 0.005 & 0.003 & 11.209 \\
\hline 18 & LISINOPRIL & SIMVASTATIN & 1737 & 1014 & 0.0256 & 0.0149 & 14.63 & 291 & 106 & 0.018 & 0.007 & 0.003 & 20.841 \\
\hline 19 & IBUPROFEN & TRAMADOL HCL & 1675 & 248 & 0.0247 & 0.0037 & 7.30 & 691 & 110 & 0.043 & 0.007 & 0.003 & 8.458 \\
\hline 20 & ALBUTEROL & PREDNISONE & 785 & 436 & 0.0116 & 0.0064 & 10.90 & 275 & 190 & 0.017 & 0.012 & 0.002 & 11.879 \\
\hline 21 & CYCLOBENZAPR & NAPROXEN & 260 & 493 & 0.0038 & 0.0073 & 60.05 & 122 & 174 & 0.008 & 0.011 & 0.002 & 28.195 \\
\hline 22 & ALBUTEROL & AZITHROMYCIN & 785 & 3180 & 0.0116 & 0.0469 & 2.76 & 275 & 891 & 0.017 & 0.056 & 0.002 & 2.352 \\
\hline 23 & HYDROCO/APAP & PENICILLN VK & 1238 & 269 & 0.0182 & 0.0040 & 18.56 & 698 & 94 & 0.044 & 0.006 & 0.002 & 8.784 \\
\hline 24 & AMOXICILLIN & PREDNISONE & 4471 & 436 & 0.0659 & 0.0064 & 2.87 & 1190 & 190 & 0.074 & 0.012 & 0.002 & 2.549 \\
\hline 25 & AMLODIPINE & HYDROCHLOROT & 684 & 463 & 0.0101 & 0.0068 & 35.35 & 81 & 85 & 0.005 & 0.005 & 0.002 & 74.053 \\
\hline 26 & CEPHALEXIN & HYDROCO/APAP & 538 & 1238 & 0.0079 & 0.0182 & 8.93 & 196 & 698 & 0.012 & 0.044 & 0.002 & 3.727 \\
\hline 27 & AMOXICILLIN & APAP/CODEINE & 4471 & 614 & 0.0659 & 0.0090 & 2.68 & 1190 & 304 & 0.074 & 0.019 & 0.002 & 1.348 \\
\hline 28 & SERTRALINE & TRAZODONE & 539 & 80 & 0.0079 & 0.0012 & 67.61 & 97 & 13 & 0.006 & 0.001 & 0.002 & 369.165 \\
\hline 29 & HYDROCO/APAP & NAPROXEN & 1238 & 493 & 0.0182 & 0.0073 & 7.07 & 698 & 174 & 0.044 & 0.011 & 0.002 & 3.650 \\
\hline 30 & HYDROCO/APAP & OXYCOD/APAP & 1238 & 324 & 0.0182 & 0.0048 & 7.27 & 698 & 175 & 0.044 & 0.011 & 0.002 & 3.630 \\
\hline
\end{tabular}

The following explains the table labels for Table 4.26 and subsequent richness tables:

- Part1 and part2 refers to the first element and the second element in the pair, respectively.

- Part1-occ and part2-occ refers to the number of occurrence for element1 and element2, respectively. This represents the times that an element occurs individually in a single round. 
- $p$ (part1-occ) and $p($ part2-occ) refers to the probability that element1 and element2 occurs individually in the dataset, respectively.

- Occ-richness is the richness measure for a pair with respect to the occurrence.

- Part1-ER Occ and part2-ER Occ refers to the number of occurrence with ER for element1 and element2, respectively. This represents the times that an element occurs individually with ER visits in a single round.

- $\quad p$ (part1-ERocc) and $p$ (part2-ERocc) refers to the probability that element1 and element2 occurs individually with ER visits in the dataset, respectively.

- $\quad P($ two-case-ER) refers to the joint probability that a pair occurs with ER visits.

- ER-richness is the richness measure for a pair with respect to ER visits.

As shown in the Table 4.25 and Table 4.26, the richness of (HYDROCO/APAP--IBUPROFEN) is equal to 60.05 and the occurrences of HYDROCO/APAP and IBUPROFEN separately are found as (CYCLOBENZAPR: 260, NAPROXEN: 493). The expected observation for their joint occurrence is defined by the product of their probability, i.e, multiplication of 0.0038 and 0.0073 . It can be observed that the actual observation of their co-occurrence is much higher than the expected observation. This means that they were found to occur together more than expected. The high value of richness for this combination reflects this conclusion. Such conclusion can be taken into consideration by health care specialists to consider the popularity of drug combinations with respect to the occurrence of the individual elements occurrences and maybe prioritize studying the impact of the co-occurrence over the single occurrence for some situations.

Observing the richness with respect to occurrence of ER events reveals that the two drugs (CYCLOBENZAPR and HYDROCO/APAP) separately have a corresponding ER value of 122, and 698 and probability 0.008 and 0.044 , respectively. Their expected observation is equal to 0.000352 . On the other hand, the probability of their joint observation was found as 0.004 . Thus, they tend to occur jointly with ER more than a parted. Again, this emphasizes the importance of monitoring the effect of their joint polypharmacy on ER more than their single polypharmacy. Moreover, it can be observed that while the pair (CYCLOBENZAPR--HYDROCO/APAP) had more richness (over-representation) than (LISINOPRIL--SIMVASTATIN) in terms of occurrence (27.90 vs. 14.63), when we consider their association with ER visits, (LISINOPRIL--SIMVASTATIN) showed a much more richness (13.536 vs. 20.84). This means that, with respect to drug safety risk, the pair (LISINOPRIL--SIMVASTATIN) may pose a significantly higher risk, when compared with (CYCLOBENZAPR--HYDROCO/APAP), even though (CYCLOBENZAPR--HYDROCO/APAP) were more over-represented with respect to their frequency of occurrence. Figure 4.13 visualizes the drug richness with respect to occurrence and ER. 
Figure 4.13 drug pair occurrence richness plotted against drug pair ER occurrence richness in log scale.

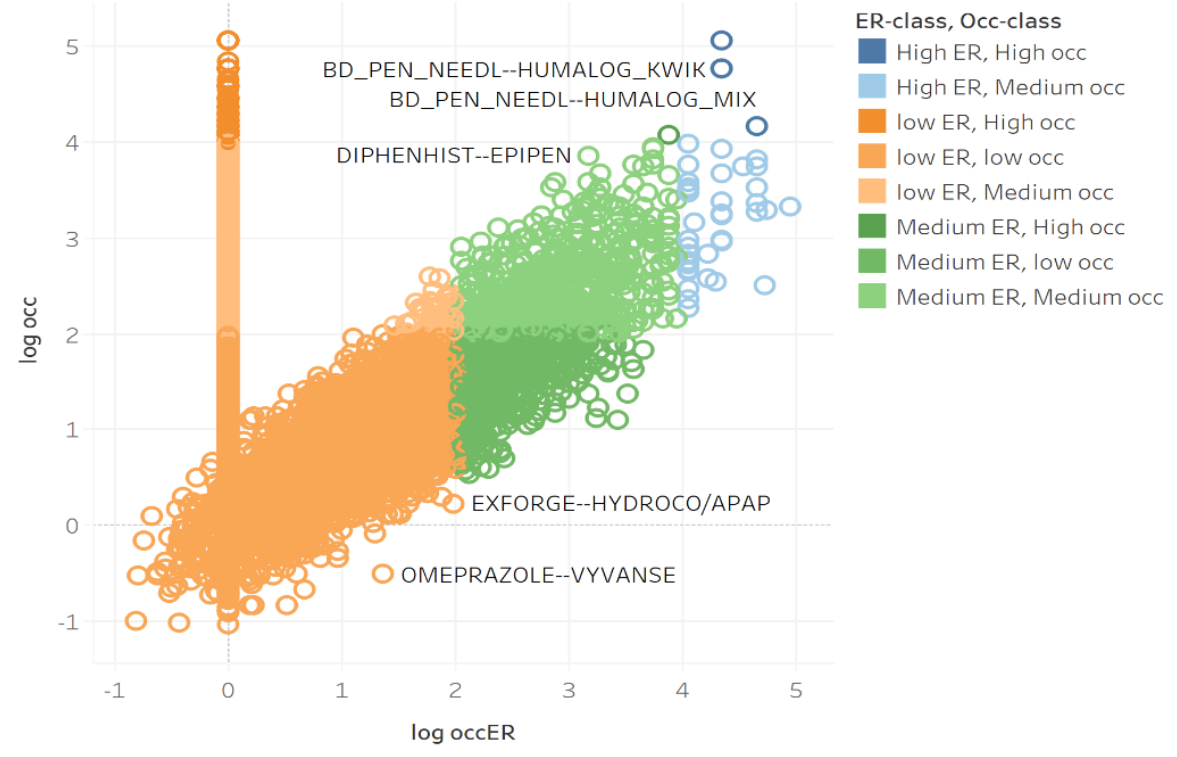

It is important in health care disciplines to look for drug associations with ER that may pose high risk. However, it is also equally important to find drugs that seem to be safe as this would help health care experts to classify them from a safety point of view. Thus, we identify three combinations for all polypharmacy definitions that would be interesting to be reviewed by health care people. This includes:

- Elements with high occurrence richness and low ER richness.

- Elements with high occurrence richness and high ER richness.

- Elements with low occurrence richness and high ER richness.

In Table 4.27 we list some of the drug pairs from the above groups. Based on the results, there were not any drug pairs found for the case (low occurrence richness and high ER richness).

Table 4.27 Examples of drug pairs with high occurrence richness and low ER richness, high occurrence richness and low ER richness, and low occurrence richness and high ER richness.

\begin{tabular}{|l|l|c|c|}
\hline Groups & Drug pairs & Occ-Richness & ER-richness \\
\hline \multirow{2}{*}{$\begin{array}{l}\text { high occurrence richness } \\
\text { and high ER richness }\end{array}$} & BD_PEN_NEEDL-HUMALOG_MIX & $14,577.15$ & $44,334.96$ \\
\cline { 2 - 4 } & $\begin{array}{l}\text { BD_PEN_NEEDL-- } \\
\text { HUMALOG_KWIK }\end{array}$ & $58,308.62$ & $22,167.48$ \\
\hline
\end{tabular}




\begin{tabular}{|l|l|c|c|}
\hline & $\begin{array}{l}\text { HYDROCHLOROT 25MG TAB-- } \\
\text { METOPROL TAR 10OMG TAB }\end{array}$ & $116,617.24$ & $22,167.48$ \\
\cline { 2 - 4 } & $\begin{array}{l}\text { MUCINEX CHLD--RESPIRATORY } \\
\text { AGENTS }\end{array}$ & $58,308.62$ & $22,167.48$ \\
\hline $\begin{array}{l}\text { high occurrence richness } \\
\text { and low ER richness }\end{array}$ & $\begin{array}{l}\text { OXYCODONE_HCL-- } \\
\text { SPIRONOLACTONE }\end{array}$ & $29,154.31$ & 0 \\
\hline & $\begin{array}{l}\text { CIPROFLOXACIN-- } \\
\text { OXYCODONE/APAP }\end{array}$ & $14,577.15$ & 0 \\
\hline & DONEPEZIL_HCL--TERAZOSIN_HCL & $58,308.62$ & 0 \\
\hline & PEDIA-LAX--SENNA & $58,308.62$ & 0 \\
\hline & $\begin{array}{l}\text { AZITHROMYCIN Z-PAK-- } \\
\text { CIPROFLOXACIN }\end{array}$ & $29,154.31$ & 0 \\
\hline & ACCU-CHEK--SOFTCLIX & $20,281.25$ & 0 \\
\hline & ALBUTEROL_HFA-_ASMANEX_30 & $29,154.31$ & 0 \\
\hline & AMITRIPTYLINE--TUSSIN_DM & $116,617.24$ & 0 \\
\hline low occurrence richness & AMMONIUM LACTATE--SOFTCLIX & $116,617.24$ & 0 \\
\hline & $\begin{array}{l}\text { ASCORBIC ACID TAB 500 MG-- } \\
\text { INTEGRA }\end{array}$ & $23,323.44$ & 0 \\
\hline & - & - & - \\
\hline
\end{tabular}

\subsubsection{Richness of Active Ingredients polypharmacy}

This section contains tables with richness values for the top 30 two-active ingredient polypharmacy sets ranked based on ER visits. Table 4.28 shows the top active ingredient-pairs and their co-occurrences probability ranked by ER total and Table $\mathbf{4 . 2 9}$ shows the individual and joint probability of occurrence of the top 30 two-active ingredient polypharmacy (sorted by ER total) with richness of occurrence and ER. 
Table 4.28: Top 30 active ingredient-pairs and their co-occurrences probability ranked by ER total.

\begin{tabular}{|c|c|c|c|c|c|}
\hline & Two-active ingredient polypharmacy & Occ & ER & \#ER/\#OCC & p(two-occ) \\
\hline 1 & $\begin{array}{l}\text { ACETAMINOPHEN--HYDROCODONE } \\
\text { BITARTRATE }\end{array}$ & 1119 & 628 & 0.561 & 0.0296 \\
\hline 2 & $\begin{array}{l}\text { ACETAMINOPHEN--CODEINE } \\
\text { PHOSPHATE }\end{array}$ & 632 & 317 & 0.502 & 0.0167 \\
\hline 3 & $\begin{array}{l}\text { AMOXICILLIN--CLAVULANATE } \\
\text { POTASSIUM }\end{array}$ & 678 & 253 & 0.373 & 0.0179 \\
\hline 4 & $\begin{array}{l}\text { ACETAMINOPHEN--OXYCODONE } \\
\text { HYDROCHLORIDE }\end{array}$ & 440 & 235 & 0.534 & 0.0116 \\
\hline 5 & $\begin{array}{l}\text { SULFAMETHOXAZOLE-- } \\
\text { TRIMETHOPRIM }\end{array}$ & 508 & 189 & 0.372 & 0.0134 \\
\hline 6 & AMOXICILLIN--IBUPROFEN & 301 & 98 & 0.326 & 0.0080 \\
\hline 7 & HYDROCHLOROTHIAZIDE--LISINOPRIL & 735 & 92 & 0.125 & 0.0194 \\
\hline 8 & ALBUTEROL SULFATE--PREDNISONE & 75 & 67 & 0.893 & 0.0020 \\
\hline 9 & AMOXICILLIN--ALBUTEROL SULFATE & 103 & 60 & 0.583 & 0.0027 \\
\hline 10 & $\begin{array}{l}\text { ALBUTEROL SULFATE--FLUTICASONE } \\
\text { PROPIONATE }\end{array}$ & 190 & 56 & 0.295 & 0.0050 \\
\hline 11 & $\begin{array}{l}\text { CYCLOBENZAPRINE HYDROCHLORIDE-- } \\
\text { IBUPROFEN }\end{array}$ & 98 & 56 & 0.571 & 0.0026 \\
\hline 12 & $\begin{array}{l}\text { AZITHROMYCIN DIHYDRATE-- } \\
\text { IBUPROFEN }\end{array}$ & 122 & 47 & 0.385 & 0.0032 \\
\hline 13 & IBUPROFEN--PREDNISONE & 14 & 46 & 3.286 & 0.0004 \\
\hline 14 & AMLODIPINE BESYLATE--LISINOPRIL & 147 & 43 & 0.293 & 0.0039 \\
\hline 15 & $\begin{array}{l}\text { AZITHROMYCIN DIHYDRATE-- } \\
\text { PREDNISONE }\end{array}$ & 63 & 36 & 0.571 & 0.0017 \\
\hline 16 & $\begin{array}{l}\text { ETHINYL ESTRADIOL-- } \\
\text { NORETHINDRONE ACETATE }\end{array}$ & 311 & 36 & 0.116 & 0.0082 \\
\hline 17 & ALBUTEROL SULFATE--BUDESONIDE & 100 & 33 & 0.330 & 0.0026 \\
\hline 18 & $\begin{array}{l}\text { CYCLOBENZAPRINE HYDROCHLORIDE-- } \\
\text { NAPROXEN }\end{array}$ & 74 & 33 & 0.446 & 0.002 \\
\hline 19 & $\begin{array}{l}\text { IBUPROFEN--TRAMADOL } \\
\text { HYDROCHLORIDE }\end{array}$ & 31 & 33 & 1.065 & 0.0008 \\
\hline 20 & $\begin{array}{l}\text { AMOXICILLIN--AZITHROMYCIN } \\
\text { DIHYDRATE }\end{array}$ & 63 & 32 & 0.508 & 0.0017 \\
\hline 21 & $\begin{array}{l}\text { SERTRALINE HYDROCHLORIDE-- } \\
\text { TRAZODONE HYDROCHLORIDE }\end{array}$ & 30 & 31 & 1.033 & 0.0008 \\
\hline 22 & $\begin{array}{l}\text { HYDROCODONE BITARTRATE-- } \\
\text { HOMATROPINE METHYLBROMIDE }\end{array}$ & 94 & 30 & 0.319 & 0.0025 \\
\hline 23 & $\begin{array}{l}\text { ALBUTEROL SULFATE--MONTELUKAST } \\
\text { SODIUM }\end{array}$ & 138 & 29 & 0.210 & 0.0036 \\
\hline 24 & ALBUTEROL SULFATE--PREDNISOLONE & 52 & 29 & 0.558 & 0.0014 \\
\hline 25 & ATENOLOL--CHLORTHALIDONE & 145 & 29 & 0.200 & 0.0038 \\
\hline 26 & LISINOPRIL--SIMVASTATIN & 222 & 29 & 0.131 & 0.0059 \\
\hline
\end{tabular}




\begin{tabular}{|c|l|c|c|c|c|}
\hline 27 & $\begin{array}{l}\text { ALBUTEROL SULFATE-- } \\
\text { BECLOMETHASONE DIPROPIONATE }\end{array}$ & 104 & 28 & 0.269 & 0.0027 \\
\hline 28 & ETONOGESTREL--ETHINYL ESTRADIOL & 248 & 28 & 0.113 & 0.0066 \\
\hline 29 & $\begin{array}{l}\text { AMLODIPINE BESYLATE-- } \\
\text { HYDROCHLOROTHIAZIDE }\end{array}$ & 113 & 26 & 0.230 & 0.003 \\
\hline 30 & AMOXICILLIN--PREDNISONE & 49 & 26 & 0.531 & 0.0013 \\
\hline
\end{tabular}

Table 4.29: Individual and joint probability of occurrence for the top 30 two-active ingredient polypharmacy (sorted by ER total) with richness of occurrence and ER.

\begin{tabular}{|c|c|c|c|c|c|c|c|c|c|c|c|c|c|}
\hline & part1 & part2 & $\begin{array}{l}\text { part1- } \\
\text { occ }\end{array}$ & $\begin{array}{l}\text { part2- } \\
\text { occ }\end{array}$ & $\begin{array}{l}\text { p(part1- } \\
\text { occ) }\end{array}$ & $\begin{array}{l}\text { p(part2- } \\
\text { occ) }\end{array}$ & $\begin{array}{l}\text { Occ- } \\
\text { richness }\end{array}$ & $\begin{array}{c}\text { part1- } \\
\text { ER } \\
\text { Occ }\end{array}$ & $\begin{array}{c}\text { part2- } \\
\text { ER } \\
\text { Occ }\end{array}$ & $\begin{array}{l}\text { p(part1- } \\
\text { ERocc) }\end{array}$ & $\begin{array}{l}\text { p(part2- } \\
\text { ERocc) }\end{array}$ & $\begin{array}{c}\text { p( } \\
\text { two- } \\
\text { case- } \\
\text { ER) }\end{array}$ & $\begin{array}{l}\text { ER- } \\
\text { richness }\end{array}$ \\
\hline 1 & ACETAMINOPHEN & $\begin{array}{l}\text { HYDROCODONE } \\
\text { BITARTRATE }\end{array}$ & 308 & 47 & 0.0052 & 0.0008 & 7109.37 & 136 & 13 & 0.010 & 0.001 & 0.060 & 6133.93 \\
\hline 2 & ACETAMINOPHEN & $\begin{array}{l}\text { CODEINE } \\
\text { PHOSPHATE }\end{array}$ & 308 & 0 & 0.0052 & 0.0000 & 0.00 & 136 & 0 & 0.010 & 0.000 & 0.030 & 0.00 \\
\hline 3 & AMOXICILLIN & $\begin{array}{l}\text { CLAVULANATE } \\
\text { POTASSIUM }\end{array}$ & 4505 & 0 & 0.0764 & 0.0000 & 0.00 & 1197 & 0 & 0.089 & 0.000 & 0.024 & 0.00 \\
\hline 4 & ACETAMINOPHEN & $\begin{array}{l}\text { OXYCODONE } \\
\text { HYDROCHLORIDE }\end{array}$ & 308 & 255 & 0.0052 & 0.0043 & 515.24 & 136 & 140 & 0.010 & 0.010 & 0.022 & 213.14 \\
\hline 5 & SULFAMETHOXAZOLE & TRIMETHOPRIM & 0 & 15 & 0.0000 & 0.0003 & 0.00 & 0 & 9 & 0.000 & 0.001 & 0.018 & 0.00 \\
\hline 6 & AMOXICILLIN & IBUPROFEN & 4505 & 1696 & 0.0764 & 0.0287 & 3.62 & 1197 & 696 & 0.089 & 0.052 & 0.009 & 2.03 \\
\hline 7 & HYDROCHLOROTHIAZIDE & LISINOPRIL & 527 & 1737 & 0.0089 & 0.0294 & 73.85 & 100 & 291 & 0.007 & 0.022 & 0.009 & 54.60 \\
\hline 8 & $\begin{array}{l}\text { ALBUTEROL } \\
\text { SULFATE }\end{array}$ & PREDNISONE & 1875 & 438 & 0.0318 & 0.0074 & 8.40 & 556 & 191 & 0.041 & 0.014 & 0.006 & 10.90 \\
\hline 9 & AMOXICILLIN & $\begin{array}{l}\text { ALBUTEROL } \\
\text { SULFATE }\end{array}$ & 4505 & 1875 & 0.0764 & 0.0318 & 1.12 & 1197 & 556 & 0.089 & 0.041 & 0.006 & 1.56 \\
\hline 10 & $\begin{array}{l}\text { ALBUTEROL } \\
\text { SULFATE }\end{array}$ & $\begin{array}{l}\text { FLUTICASONE } \\
\text { PROPIONATE }\end{array}$ & 1875 & 627 & 0.0318 & 0.0106 & 14.86 & 556 & 86 & 0.041 & 0.006 & 0.005 & 20.22 \\
\hline 11 & $\begin{array}{l}\text { CYCLOBENZAPRINE } \\
\text { HYDROCHLORIDE }\end{array}$ & IBUPROFEN & 270 & 1696 & 0.0046 & 0.0287 & 19.68 & 125 & 696 & 0.009 & 0.052 & 0.005 & 11.12 \\
\hline 12 & $\begin{array}{l}\text { AZITHROMYCIN } \\
\text { DIHYDRATE }\end{array}$ & IBUPROFEN & 3233 & 1696 & 0.0548 & 0.0287 & 2.05 & 909 & 696 & 0.067 & 0.052 & 0.004 & 1.28 \\
\hline 13 & IBUPROFEN & PREDNISONE & 1696 & 438 & 0.0287 & 0.0074 & 1.73 & 696 & 191 & 0.052 & 0.014 & 0.004 & 5.98 \\
\hline 14 & $\begin{array}{l}\text { AMLODIPINE } \\
\text { BESYLATE }\end{array}$ & LISINOPRIL & 722 & 1737 & 0.0122 & 0.0294 & 10.78 & 91 & 291 & 0.007 & 0.022 & 0.004 & 28.04 \\
\hline 15 & $\begin{array}{l}\text { AZITHROMYCIN } \\
\text { DIHYDRATE }\end{array}$ & PREDNISONE & 3233 & 438 & 0.0548 & 0.0074 & 4.09 & 909 & 191 & 0.067 & 0.014 & 0.003 & 3.58 \\
\hline 16 & $\begin{array}{l}\text { ETHINY } \\
\text { LESTRADIOL }\end{array}$ & $\begin{array}{l}\text { NORETHINDRONE } \\
\text { ACETATE }\end{array}$ & 0 & 10 & 0.0000 & 0.0002 & 0.00 & 0 & 1 & 0.000 & 0.000 & 0.003 & 0.00 \\
\hline 17 & $\begin{array}{l}\text { ALBUTEROL } \\
\text { SULFATE }\end{array}$ & BUDESONIDE & 1875 & 64 & 0.0318 & 0.0011 & 76.64 & 556 & 11 & 0.041 & 0.001 & 0.003 & 93.18 \\
\hline 18 & $\begin{array}{l}\text { CYCLOBENZAPRINE } \\
\text { HYDROCHLORIDE }\end{array}$ & NAPROXEN & 270 & 499 & 0.004577 & 0.0085 & 50.51 & 125 & 175 & 0.009 & 0.013 & 0.003 & 26.05 \\
\hline 19 & IBUPROFEN & $\begin{array}{l}\text { TRAMADOL } \\
\text { HYDROCHLORIDE }\end{array}$ & 1696 & 293 & 0.028749 & 0.0050 & 5.74 & 696 & 123 & 0.052 & 0.009 & 0.003 & 6.66 \\
\hline 20 & AMOXICILLIN & $\begin{array}{l}\text { AZITHROMYCIN } \\
\text { DIHYDRATE }\end{array}$ & 4505 & 3233 & 0.076365 & 0.0548 & 0.40 & 1197 & 909 & 0.089 & 0.067 & 0.003 & 0.51 \\
\hline 21 & $\begin{array}{l}\text { SERTRALINE } \\
\text { HYDROCHLORIDE }\end{array}$ & $\begin{array}{l}\text { TRAZODONE } \\
\text { HYDROCHLORIDE }\end{array}$ & 603 & 80 & 0.010222 & 0.0014 & 57.20 & 106 & 13 & 0.008 & 0.001 & 0.003 & 388.48 \\
\hline 22 & $\begin{array}{l}\text { HYDROCODONE } \\
\text { BITARTRATE }\end{array}$ & $\begin{array}{l}\text { HOMATROPINE } \\
\text { METHYLBROMIDE }\end{array}$ & 47 & 0 & 0.000797 & 0.0000 & 0.00 & 13 & 0 & 0.001 & 0.000 & 0.003 & 0.00 \\
\hline
\end{tabular}




\begin{tabular}{|c|c|c|c|c|c|c|c|c|c|c|c|c|c|}
\hline 23 & $\begin{array}{l}\text { ALBUTEROL } \\
\text { SULFATE }\end{array}$ & $\begin{array}{l}\text { MONTELUKAST } \\
\text { SODIUM }\end{array}$ & 1875 & 452 & 0.031783 & 0.0077 & 14.98 & 556 & 95 & 0.041 & 0.007 & 0.003 & 9.48 \\
\hline 24 & $\begin{array}{l}\text { ALBUTEROL } \\
\text { SULFATE }\end{array}$ & PREDNISOLONE & 1875 & 151 & 0.031783 & 0.0026 & 16.89 & 556 & 54 & 0.041 & 0.004 & 0.003 & 16.68 \\
\hline 25 & ATENOLOL & CHLORTHALIDONE & 447 & 22 & 0.007577 & 0.0004 & 1356.09 & 66 & 5 & 0.005 & 0.000 & 0.003 & 1517.56 \\
\hline 26 & LISINOPRIL & SIMVASTATIN & 1737 & 1022 & 0.029444 & 0.0173 & 11.50 & 291 & 107 & 0.022 & 0.008 & 0.003 & 16.08 \\
\hline 27 & $\begin{array}{l}\text { ALBUTEROL } \\
\text { SULFATE }\end{array}$ & $\begin{array}{l}\text { BECLOMETHASONE } \\
\text { DIPROPIONATE }\end{array}$ & 1875 & 62 & 0.031783 & 0.0011 & 82.28 & 556 & 17 & 0.041 & 0.001 & 0.003 & 51.16 \\
\hline 28 & ETONOGESTREL & $\begin{array}{l}\text { ETHINYL } \\
\text { ESTRADIOL }\end{array}$ & 1 & 0 & $1.7 \mathrm{E}-05$ & 0.0000 & 0.00 & 0 & 0 & 0.000 & 0.000 & 0.003 & 0.00 \\
\hline 29 & $\begin{array}{l}\text { AMLODIPINE } \\
\text { BESYLATE }\end{array}$ & HYDROCHLOROTHIAZIDE & 722 & 527 & 0.012239 & 0.0089 & 27.31 & 91 & 100 & 0.007 & 0.007 & 0.002 & 49.34 \\
\hline 30 & AMOXICILLIN & PREDNISONE & 4505 & 438 & 0.076365 & 0.0074 & 2.28 & 1197 & 191 & 0.089 & 0.014 & 0.002 & 1.96 \\
\hline
\end{tabular}

The values (0) indicates that those active ingredients were not found within single occurrences of ingredients, for those the value of richness cannot be determined. A noticeable finding here is that although the pair (BETAMETHASONE DIPROPIONATE--CLOTRIMAZOLE) has much lower richness value with respect to occurrence when it is compared to its occurrence with ER (1820.25 vs. 2437.94). This implies that pair tend to be associated with high risk when it occurs.

In some cases, the richness measure provides additional insight about the safety of two ingredients than the ranking based on ER visits (provided previously in section 4.2). As we discussed earlier, drug polypharmacy may not highlight the problems resulting from single drugs. For this case the active ingredients approach to defining polypharmacy could be more informative and provide a more detailed view of looking at substances and their associations with health problems. Table $\mathbf{4 . 3 0}$ show few examples of single drugs along with their original ranking based on ER visits for patients, the equivalent active ingredients along with their original ranking based on the ER visits, and finally, the richness measure for these ingredients. It can be observed that even though (CIPROFLOXACIN HYDROCHLORIDE--DEXAMETHASONE), (HYDROCHLOROTHIAZIDE --LOSARTAN POTASSIUM), and (ATENOLOL--CHLORTHALIDONE) ingredients are ranked relatively low based on ER visits sort, they have relatively high richness. This indicates that they jointly tend to have more association with ER than what is expected from the occurrence of individual ingredients. This observation can only be derived by the richness measure.

Table 4.30 Comparison of some single drugs and their continent active ingredients ranking based on ER visits along with the richness of the active ingredient pairs.

\begin{tabular}{|c|l|c|}
\hline Single drug & Multiple AI & ER-Richness \\
\hline JANUMET ranked 929 by ER. & $\begin{array}{l}\text { SITAGLIPTIN PHOSPHATE--METFORMIN } \\
\text { HYDROCHLORIDE (ranked 554 by ER) }\end{array}$ & 47.18 \\
\hline COMBIGAN ranked 686 by ER. & $\begin{array}{l}\text { BRIMONIDINE TARTRATE--TIMOLOL } \\
\text { MALEATE (ranked 563 by ER) }\end{array}$ & - \\
\hline DYMISTA ranked 1052 by ER. & $\begin{array}{l}\text { AZELASTINEHYDROCHLORIDE-- } \\
\text { FLUTICASONE PROPIONATE (ranked } 907 \text { by } \\
\text { ER) }\end{array}$ & 80.31 \\
\hline
\end{tabular}




\begin{tabular}{|c|l|c|}
\hline CIPRODEX ranked 168 by ER. & $\begin{array}{l}\text { CIPROFLOXACIN HYDROCHLORIDE-- } \\
\text { DEXAMETHASONE (ranked 57 by ER) }\end{array}$ & 259.03 \\
\hline HYZAAR ranked 2078 by ER. & $\begin{array}{l}\text { HYDROCHLOROTHIAZIDE--LOSARTAN } \\
\text { POTASSIUM (ranked 40 by ER) }\end{array}$ & 86.34 \\
\hline $\begin{array}{c}\text { ATENOLOL/CHLORTHALIDONE } \\
\text { ranked } 1389 \text { by ER. }\end{array}$ & $\begin{array}{c}\text { ATENOLOL--CHLORTHALIDONE (ranked 27 } \\
\text { by ER) }\end{array}$ & 1517.55 \\
\hline
\end{tabular}

We use the same groups we identified above in Section 4.4 .1 to get some of the combinations that would be interesting to be reviewed by health care people. Again, this includes:

- Elements with high occurrence richness and low ER richness.

- Elements with high occurrence richness and high ER richness.

- Elements with low occurrence richness and high ER richness.

In Table 4.31 we list some of the active ingredient pairs from the above groups. Based on the richness results, only one active ingredient pair found for the case (low occurrence richness and high ER richness).

Table 4.31 Examples of active ingredient pairs with high occurrence richness and low ER richness, high occurrence richness and low ER richness, and low occurrence richness and high ER richness.

\begin{tabular}{|c|c|c|c|}
\hline Groups & Active ingredient pairs & Occ-Richness & ER-richness \\
\hline \multirow{7}{*}{$\begin{array}{l}\text { high occurrence richness } \\
\text { and high ER richness }\end{array}$} & $\begin{array}{l}\text { ACETAMINOPHEN-- } \\
\text { HYDROCODONE BITARTRATE }\end{array}$ & $7,109.37$ & $6,133.92$ \\
\hline & $\begin{array}{l}\text { FLUTICASONE PROPIONATE-- } \\
\text { SALMETEROL XINAFOATE }\end{array}$ & $32,123.76$ & 2,309.19 \\
\hline & $\begin{array}{l}\text { BISOPROLOL FUMARATE-- } \\
\text { HYDROCHLOROTHIAZIDE }\end{array}$ & $3,730.31$ & $1,381.50$ \\
\hline & DEXAMETHASONE--TOBRAMYCIN & $2,556.25$ & $1,569.88$ \\
\hline & $\begin{array}{l}\text { HYDROCORTISONE ACETATE-- } \\
\text { PRAMOXINE HYDROCHLORIDE }\end{array}$ & $36,131.37$ & $60,440.69$ \\
\hline & $\begin{array}{l}\text { DEXTROMETHORPHAN } \\
\text { HYDROBROMIDE--GUAIFENESIN }\end{array}$ & $12,358.57$ & $3,139.77$ \\
\hline & $\begin{array}{l}\text { CALCIUM ACETATE--SEVELAMER } \\
\text { CARBONATE }\end{array}$ & $137,956.15$ & $1,439.06$ \\
\hline
\end{tabular}




\begin{tabular}{|c|c|c|c|}
\hline & $\begin{array}{l}\text { ZIPRASIDONE MESYLATE-- } \\
\text { ZIPRASIDONE HYDROCHLORIDE }\end{array}$ & $4,379.56$ & $25,903.15$ \\
\hline & $39328005750--642007630$ & $7,074.67$ & $5,756.25$ \\
\hline & 193654621--193709021 & $4,598.53$ & $2,466.96$ \\
\hline \multirow[t]{10}{*}{$\begin{array}{l}\text { high occurrence richness } \\
\text { and low ER richness }\end{array}$} & $193709021--8290328466$ & $9,197.07$ & 0 \\
\hline & $\begin{array}{l}\text { MAGNESIUM HYDROXIDE--SENNA } \\
\text { LEAF }\end{array}$ & $45,985.38$ & 0 \\
\hline & 49614043612--ZAFIRLUKAST & $10,218.97$ & 0 \\
\hline & ADAPALENE--BENZOYL PEROXIDE & $12,411.28$ & 0 \\
\hline & DOCUSATE SODIUM--SENNOSIDES & $15,328.46$ & 0 \\
\hline & $\begin{array}{l}\text { DORZOLAMIDE HYDROCHLORIDE-- } \\
\text { TIMOLOL MALEATE }\end{array}$ & $34,489.03$ & 0 \\
\hline & 50924098850--50924097110 & $61,313.84$ & 0 \\
\hline & 182101701--MODAFINIL & $10,218.97$ & 0 \\
\hline & 677007010--99073070805 & $9,197.07$ & 0 \\
\hline & 38142300000--54838014530 & $18,394.15$ & 0 \\
\hline $\begin{array}{l}\text { low occurrence richness } \\
\text { and high ER richness }\end{array}$ & GEMFIBROZIL--LOVASTATIN & 9.88933 & $2,072.25$ \\
\hline
\end{tabular}


We plot the trend of active ingredients richness with respect to occurrence and ER in Figure 4.14.

Figure 4.14 Relationship between active ingredients occurrence and ER richness using a log scale (logarithms are in base 10).

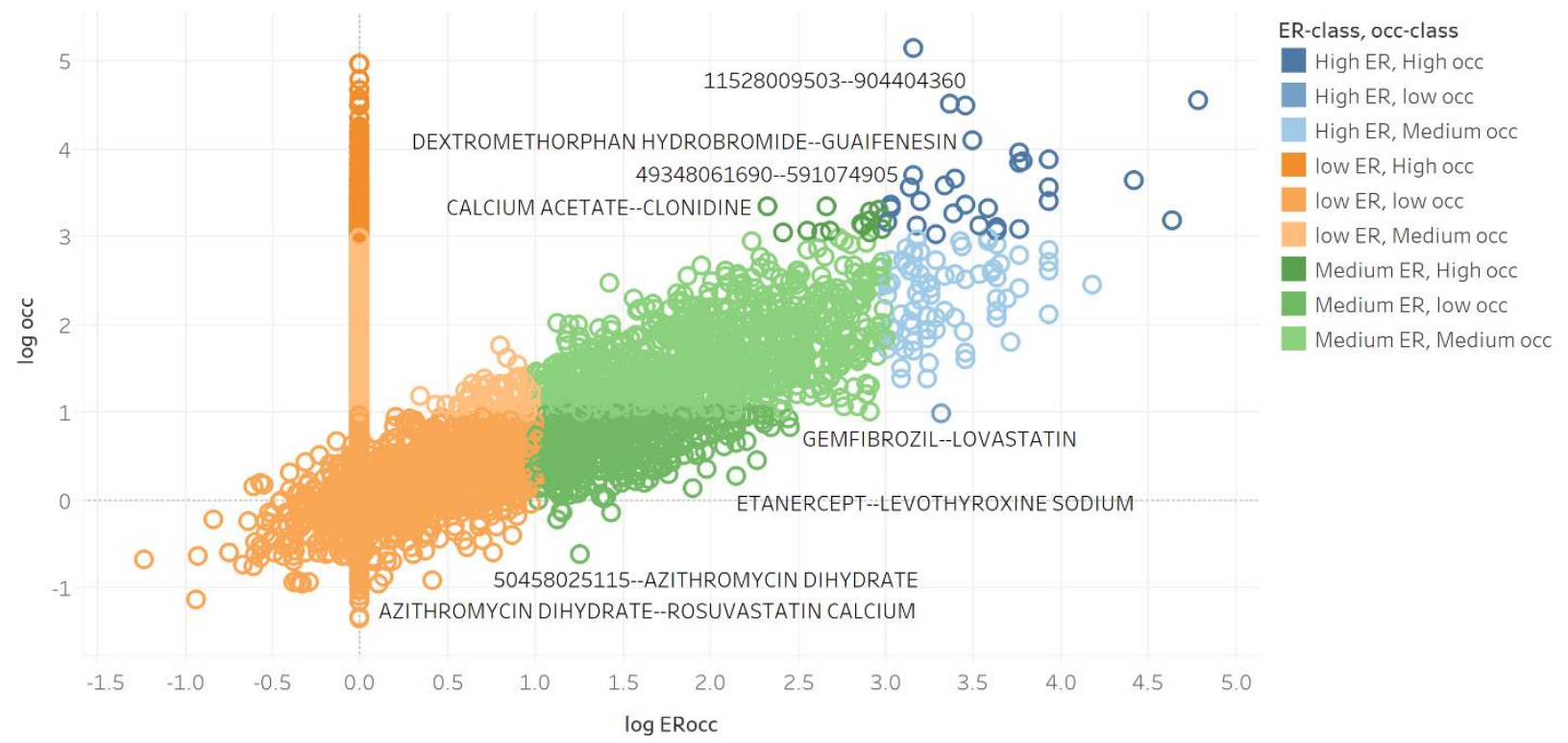

\subsubsection{Richness of drug classes polypharmacy}

This section contains tables with richness values for the top 30 two-TC1 drug class polypharmacy sets ranked based on ER visits. Table 4.32 shows the top TC1 class -pairs and their co-occurrences probability ranked by ER total and Table $\mathbf{4 . 3 3}$ shows the individual and joint probability of occurrence of the top 30 two-TC1 class polypharmacy (sorted by ER total) with richness of occurrence and ER.

Table 4.32: Top 30 TC1 class-pairs and their co-occurrences probability ranked by ER total.

\begin{tabular}{|c|c|c|c|c|c|}
\hline & TC1 class pairs & occ-Total & ER-Total & $\begin{array}{l}\text { \#ER/ } \\
\text { \#Occ }\end{array}$ & $\begin{array}{c}\text { p(two- } \\
\text { occ) }\end{array}$ \\
\hline 1 & Anti_infectives--Central nervous system agents & 3,762 & 1,856 & 0.49 & 0.08 \\
\hline 2 & Cardiovascular agents-- Metabolic agents & 8,224 & 1,496 & 0.18 & 0.17 \\
\hline 3 & Central nervous system agents--Psychotherapeutic agents & 3,072 & 1,160 & 0.38 & 0.06 \\
\hline 4 & Central nervous system agents--Cardiovascular agents & 1,663 & 686 & 0.41 & 0.03 \\
\hline 5 & Anti_infectives--Respiratory agents & 1,893 & 673 & 0.36 & 0.04 \\
\hline 6 & Central nervous system agents--Hormones/hormone modifiers & 1,461 & 570 & 0.39 & 0.03 \\
\hline 7 & Central nervous system agents--Gastrointestinal agents & 1,091 & 544 & 0.50 & 0.02 \\
\hline 8 & Central nervous system agents--Respiratory agents & 1,275 & 541 & 0.42 & 0.03 \\
\hline 9 & Anti_infectives--Topical agents & 1,800 & 479 & 0.27 & 0.04 \\
\hline 10 & Cardiovascular agents--Central nervous system agents & 1,380 & 447 & 0.32 & 0.03 \\
\hline
\end{tabular}




\begin{tabular}{|l|l|c|c|c|c|}
\hline 11 & Central nervous system agents--Topical agents & 1,111 & 409 & 0.37 & 0.02 \\
\hline 12 & Central nervous system agents-- Metabolic agents & 1,196 & 362 & 0.30 & 0.03 \\
\hline 13 & Hormones/hormone modifiers--Respiratory agents & 835 & 326 & 0.39 & 0.02 \\
\hline 14 & Respiratory agents--Topical agents & 1,197 & 317 & 0.26 & 0.03 \\
\hline 15 & Anti_infectives--Hormones/hormone modifiers & 1,075 & 316 & 0.29 & 0.02 \\
\hline 16 & Central nervous system agents--Nutritional products & 602 & 303 & 0.50 & 0.01 \\
\hline 17 & Cardiovascular agents--Gastrointestinal agents & 830 & 236 & 0.28 & 0.02 \\
\hline 18 & Anti_infectives--Cardiovascular agents & 736 & 221 & 0.30 & 0.02 \\
\hline 19 & Cardiovascular agents--Hormones/hormone modifiers & 1,335 & 214 & 0.16 & 0.03 \\
\hline 20 & Cardiovascular agents--Psychotherapeutic agents & 752 & 185 & 0.25 & 0.02 \\
\hline 21 & Cardiovascular agents--Nutritional products & 501 & 179 & 0.36 & 0.01 \\
\hline 22 & Cardiovascular agents--Topical agents & 651 & 167 & 0.26 & 0.01 \\
\hline 23 & Hormones/hormone modifiers--Topical agents & 631 & 138 & 0.22 & 0.01 \\
\hline 24 & Cardiovascular agents--Respiratory agents & 504 & 131 & 0.26 & 0.01 \\
\hline 25 & Anti_infectives-- Metabolic agents & 347 & 117 & 0.34 & 0.01 \\
\hline 26 & Anti_infectives--Nutritional products & 277 & 114 & 0.41 & 0.01 \\
\hline 27 & Anti_infectives--Gastrointestinal agents & 322 & 112 & 0.35 & 0.01 \\
\hline 28 & Hormones/hormone modifiers--Psychotherapeutic agents & 577 & 108 & 0.19 & 0.01 \\
\hline 29 & Hormones/hormone modifiers--Nutritional products & 184 & 97 & 1 & 0.004 \\
\hline 30 & Gastrointestinal agents--Nutritional products & 144 & 95 & 1 & 0.003 \\
\hline
\end{tabular}

Table 4.33: Individual and joint probability of occurrence for the top 30 two-TC1 class polypharmacy (sorted by ER total) with richness of occurrence and ER.

\begin{tabular}{|l|l|c|c|c|c|c|c|c|c|c|c|c|}
\hline \multicolumn{1}{|c|}{ TC1 class pairs } & $\begin{array}{c}\text { part1- } \\
\text { occ }\end{array}$ & $\begin{array}{c}\text { p(part1- } \\
\text { occ) }\end{array}$ & $\begin{array}{c}\text { part2- } \\
\text { occ }\end{array}$ & $\begin{array}{c}\text { p(part2- } \\
\text { occ) }\end{array}$ & $\begin{array}{c}\text { occ- } \\
\text { richness }\end{array}$ & $\begin{array}{c}\text { part1- } \\
\text { ERocc }\end{array}$ & $\begin{array}{c}\text { p(part1- } \\
\text { ERocc }\end{array}$ & $\begin{array}{c}\text { part2- } \\
\text { ERocc }\end{array}$ & $\begin{array}{c}\text { p(part2- } \\
\text { ERocc) }\end{array}$ & $\begin{array}{c}\text { (two- } \\
\text { case- } \\
\text { ER) }\end{array}$ & $\begin{array}{c}\text { ER- } \\
\text { richness }\end{array}$ \\
\hline $\begin{array}{l}\text { Anti_infectives-- } \\
\text { Central nervous } \\
\text { system agents }\end{array}$ & 13495 & 0.17 & 17160 & 0.22 & 2.17 & 4053 & 0.1878 & 6792 & 0.3147 & 0.1247 & 2.11 \\
\hline $\begin{array}{l}\text { Cardiovascular } \\
\text { agents-- Metabolic } \\
\text { agents }\end{array}$ & 10883 & 0.14 & 5914 & 0.07 & 17.07 & 2245 & 0.1040 & 1049 & 0.0486 & 0.1005 & 19.89 \\
\hline $\begin{array}{l}\text { Central nervous } \\
\text { system agents-- } \\
\text { Psychotherapeutic } \\
\text { agents }\end{array}$ & 17160 & 0.22 & 3379 & 0.04 & 7.08 & 6792 & 0.3147 & 831 & 0.0385 & 0.0780 & 6.44 \\
\hline
\end{tabular}




\begin{tabular}{|c|c|c|c|c|c|c|c|c|c|c|c|c|}
\hline 4 & $\begin{array}{l}\text { Central nervous } \\
\text { system agents-- } \\
\text { Cardiovascular } \\
\text { agents }\end{array}$ & 17160 & 0.22 & 10883 & 0.14 & 1.19 & 6792 & 0.3147 & 2245 & 0.1040 & 0.0461 & 1.41 \\
\hline 5 & $\begin{array}{l}\text { Anti_infectives-- } \\
\text { Respiratory agents }\end{array}$ & 13495 & 0.17 & 6407 & 0.08 & 2.93 & 4053 & 0.1878 & 1647 & 0.0763 & 0.0452 & 3.16 \\
\hline 6 & $\begin{array}{l}\text { Central nervous } \\
\text { system agents-- } \\
\text { Hormones/hormone } \\
\text { modifiers }\end{array}$ & 17160 & 0.22 & 8787 & 0.11 & 1.29 & 6792 & 0.3147 & 1491 & 0.0691 & 0.0383 & 1.76 \\
\hline 7 & $\begin{array}{l}\text { Central nervous } \\
\text { system agents-- } \\
\text { Gastrointestinal } \\
\text { agents }\end{array}$ & 17160 & 0.22 & 2561 & 0.03 & 3.32 & 6792 & 0.3147 & 714 & 0.0331 & 0.0366 & 3.51 \\
\hline 8 & $\begin{array}{l}\text { Central nervous } \\
\text { system agents-- } \\
\text { Respiratory agents }\end{array}$ & 17160 & 0.22 & 6407 & 0.08 & 1.55 & 6792 & 0.3147 & 1647 & 0.0763 & 0.0364 & 1.51 \\
\hline 9 & $\begin{array}{l}\text { Anti_infectives-- } \\
\text { Topical agents }\end{array}$ & 13495 & 0.17 & 6588 & 0.08 & 2.71 & 4053 & 0.1878 & 1492 & 0.0691 & 0.0322 & 2.48 \\
\hline 10 & $\begin{array}{l}\text { Cardiovascular } \\
\text { agents--Central } \\
\text { nervous system } \\
\text { agents }\end{array}$ & 10883 & 0.14 & 17160 & 0.22 & 0.99 & 2245 & 0.1040 & 6792 & 0.3147 & 0.0300 & 0.92 \\
\hline 11 & $\begin{array}{l}\text { Central nervous } \\
\text { system agents-- } \\
\text { Topical agents }\end{array}$ & 17160 & 0.22 & 6588 & 0.08 & 1.31 & 6792 & 0.3147 & 1492 & 0.0691 & 0.0275 & 1.26 \\
\hline 12 & $\begin{array}{l}\text { Central nervous } \\
\text { system agents-- } \\
\text { Metabolic agents }\end{array}$ & 17160 & 0.22 & 5914 & 0.07 & 1.57 & 6792 & 0.3147 & 1049 & 0.0486 & 0.0243 & 1.59 \\
\hline 13 & $\begin{array}{l}\text { Hormones/hormone } \\
\text { modifiers-- } \\
\text { Respiratory agents }\end{array}$ & 8787 & 0.11 & 6407 & 0.08 & 1.98 & 1491 & 0.0691 & 1647 & 0.0763 & 0.0219 & 4.16 \\
\hline
\end{tabular}




\begin{tabular}{|c|c|c|c|c|c|c|c|c|c|c|c|c|}
\hline 14 & $\begin{array}{l}\text { Respiratory agents-- } \\
\text { Topical agents }\end{array}$ & 6407 & 0.08 & 6588 & 0.08 & 3.79 & 1647 & 0.0763 & 1492 & 0.0691 & 0.0213 & 4.04 \\
\hline 15 & $\begin{array}{l}\text { Anti_infectives-- } \\
\text { Hormones/hormone } \\
\text { modifiers }\end{array}$ & 13495 & 0.17 & 8787 & 0.11 & 1.21 & 4053 & 0.1878 & 1491 & 0.0691 & 0.0212 & 1.64 \\
\hline 16 & $\begin{array}{l}\text { Central nervous } \\
\text { system agents-- } \\
\text { Nutritional products }\end{array}$ & 17160 & 0.22 & 2258 & 0.03 & 2.08 & 6792 & 0.3147 & 669 & 0.0310 & 0.0204 & 2.09 \\
\hline 17 & $\begin{array}{l}\text { Cardiovascular } \\
\text { agents-- } \\
\text { Gastrointestinal } \\
\text { agents }\end{array}$ & 10883 & 0.14 & 2561 & 0.03 & 3.98 & 2245 & 0.1040 & 714 & 0.0331 & 0.0159 & 4.61 \\
\hline 18 & $\begin{array}{l}\text { Anti_infectives-- } \\
\text { Cardiovascular } \\
\text { agents }\end{array}$ & 13495 & 0.17 & 10883 & 0.14 & 0.67 & 4053 & 0.1878 & 2245 & 0.1040 & 0.0149 & 0.76 \\
\hline 19 & $\begin{array}{l}\text { Cardiovascular } \\
\text { agents-- } \\
\text { Hormones/hormone } \\
\text { modifiers }\end{array}$ & 10883 & 0.14 & 8787 & 0.11 & 1.87 & 2245 & 0.1040 & 1491 & 0.0691 & 0.0144 & 2.00 \\
\hline 20 & $\begin{array}{l}\text { Cardiovascular } \\
\text { agents-- } \\
\text { Psychotherapeutic } \\
\text { agents }\end{array}$ & 10883 & 0.14 & 3379 & 0.04 & 2.73 & 2245 & 0.1040 & 831 & 0.0385 & 0.0124 & 3.10 \\
\hline 21 & $\begin{array}{l}\text { Cardiovascular } \\
\text { agents--Nutritional } \\
\text { products }\end{array}$ & 10883 & 0.14 & 2258 & 0.03 & 2.72 & 2245 & 0.1040 & 669 & 0.0310 & 0.0120 & 3.73 \\
\hline 22 & $\begin{array}{l}\text { Cardiovascular } \\
\text { agents--Topical } \\
\text { agents }\end{array}$ & 10883 & 0.14 & 6588 & 0.08 & 1.21 & 2245 & 0.1040 & 1492 & 0.0691 & 0.0112 & 1.56 \\
\hline 23 & $\begin{array}{l}\text { Hormones/hormone } \\
\text { modifiers--Topical } \\
\text { agents }\end{array}$ & 8787 & 0.11 & 6588 & 0.08 & 1.46 & 1491 & 0.0691 & 1492 & 0.0691 & 0.0093 & 1.94 \\
\hline
\end{tabular}




\begin{tabular}{|c|c|c|c|c|c|c|c|c|c|c|c|c|}
\hline 24 & $\begin{array}{l}\text { Cardiovascular } \\
\text { agents--Respiratory } \\
\text { agents }\end{array}$ & 10883 & 0.14 & 6407 & 0.08 & 0.97 & 2245 & 0.1040 & 1647 & 0.0763 & 0.0088 & 1.11 \\
\hline 25 & $\begin{array}{l}\text { Anti_infectives-- } \\
\text { Metabolic agents }\end{array}$ & 13495 & 0.17 & 5914 & 0.07 & 0.58 & 4053 & 0.1878 & 1049 & 0.0486 & 0.0079 & 0.86 \\
\hline 26 & $\begin{array}{l}\text { Anti_infectives-- } \\
\text { Nutritional products }\end{array}$ & 13495 & 0.17 & 2258 & 0.03 & 1.21 & 4053 & 0.1878 & 669 & 0.0310 & 0.0077 & 1.32 \\
\hline 27 & $\begin{array}{l}\text { Anti_infectives-- } \\
\text { Gastrointestinal } \\
\text { agents }\end{array}$ & 13495 & 0.17 & 2561 & 0.03 & 1.24 & 4053 & 0.1878 & 714 & 0.0331 & 0.0075 & 1.21 \\
\hline 28 & $\begin{array}{l}\text { Hormones/hormone } \\
\text { modifiers-- } \\
\text { Psychotherapeutic } \\
\text { agents }\end{array}$ & 8787 & 0.11 & 3379 & 0.04 & 2.60 & 1491 & 0.0691 & 831 & 0.0385 & 0.0073 & 2.73 \\
\hline 29 & $\begin{array}{l}\text { Hormones/hormone } \\
\text { modifiers--Nutritional } \\
\text { products }\end{array}$ & 8787 & 0.11 & 2258 & 0.03 & 1.24 & 1491 & 0.0691 & 669 & 0.0310 & 0.0065 & 3.04 \\
\hline 30 & $\begin{array}{l}\text { Gastrointestinal } \\
\text { agents--Nutritional } \\
\text { products }\end{array}$ & 2561 & 0.03 & 2258 & 0.03 & 3.33 & 714 & 0.0331 & 669 & 0.0310 & 0.0064 & 6.23 \\
\hline
\end{tabular}

Cardiovascular agents-Metabolic agents has lower tendency to occur together compared to their tendency to occur together with ER (17.07 vs. 19.89). This again could indicate that this pair tend to be associated with higher risk when it occurs. Figure 4.15 graphs the richness of occurrence and the richness of ER visits values for TC1 drug class pairs. 
Figure 4.15 relationship between TC1 class pairs occurrence and ER richness. Logarithms are in base 10.

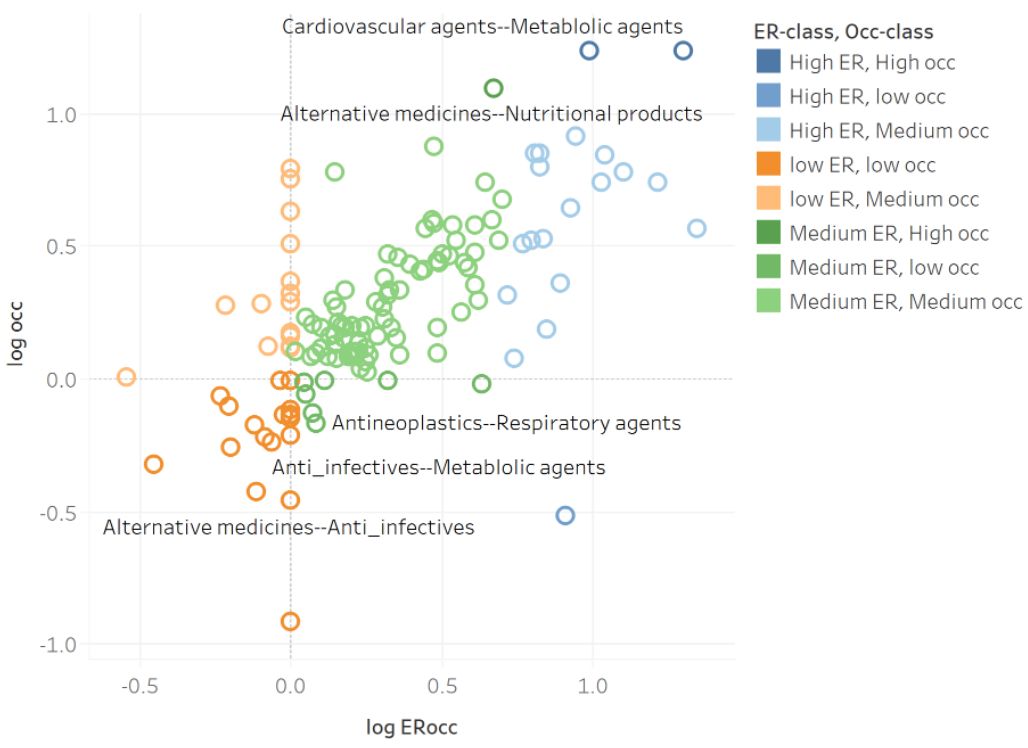

We now identify the same groups we chose for drug pairs and active ingredient pairs to get some of the TC1 drug class combinations that would be interesting to be reviewed by health care people. Again, this includes:

- Elements with high occurrence richness and low ER richness.

- Elements with high occurrence richness and high ER richness.

- Elements with low occurrence richness and high ER richness.

In Table 4.34 we list some of the TC1 class pairs from the above groups. Based on the richness results, only one TC1 class pair found for the case (low occurrence richness and high ER richness).

Table 4.34 Example of TC1 class pairs with high occurrence richness and low ER richness, high occurrence richness and low ER richness, and low occurrence richness and high ER richness.

\begin{tabular}{|l|l|c|c|}
\hline & TC1 class pairs & ER-Richness & $\begin{array}{l}\text { Occ- } \\
\text { Richness }\end{array}$ \\
\hline $\begin{array}{l}\text { high occurrence } \\
\text { richness and high ER } \\
\text { richness }\end{array}$ & $\begin{array}{l}\text { Cardiovascular agents--Metablolic } \\
\text { agents }\end{array}$ & 19.88 & 17.07 \\
\cline { 2 - 4 } & $\begin{array}{l}\text { Miscellaneous agents-- } \\
\text { Psychotherapeutic agents }\end{array}$ & 9.68 & 17.25 \\
\hline & $\begin{array}{l}\text { Nutritional products-- } \\
\text { Psychotherapeutic agents }\end{array}$ & 0.84 & 1.33 \\
\hline
\end{tabular}




\begin{tabular}{|c|c|c|c|}
\hline \multirow{8}{*}{$\begin{array}{l}\text { high occurrence } \\
\text { richness and low ER } \\
\text { richness }\end{array}$} & $\begin{array}{l}\text { Antineoplastics--Gastrointestinal } \\
\text { agents }\end{array}$ & 0.79 & 1.90 \\
\hline & $\begin{array}{l}\text { Cardiovascular agents--Immunologic } \\
\text { agents }\end{array}$ & 0.60 & 1.88 \\
\hline & $\begin{array}{l}\text { Alternative medicines-- } \\
\text { Psychotherapeutic agents }\end{array}$ & 0 & 2.08 \\
\hline & $\begin{array}{l}\text { Antineoplastics--Coagulation } \\
\text { modifiers }\end{array}$ & 0 & 6.19 \\
\hline & $\begin{array}{l}\text { Antineoplastics-- Genitourinary tract } \\
\text { agents }\end{array}$ & 0 & 1.49 \\
\hline & $\begin{array}{l}\text { Biologicals--Central nervous system } \\
\text { agents }\end{array}$ & 0 & 1.94 \\
\hline & $\begin{array}{l}\text { Coagulation modifiers-- } \\
\text { Genitourinary tract agents }\end{array}$ & 0 & 2.33 \\
\hline & $\begin{array}{l}\text { Genitourinary tract agents-- } \\
\text { Immunologic agents }\end{array}$ & 0 & 5.67 \\
\hline $\begin{array}{l}\text { low occurrence } \\
\text { richness and High ER } \\
\text { richness }\end{array}$ & $\begin{array}{l}\text { Gastrointestinal agents-- } \\
\text { Genitourinary tract agents }\end{array}$ & 8.12 & 0.30 \\
\hline
\end{tabular}

Table 4.35 summarizes the occurrence richness and the ER richness. We can observe from table 4.36 that the occurrence richness and the ER richness have very similar values for the case of TC1 class definition. This follows our previous discussion in Section 4.2.3 (prevalence of drug class polypharmacy) that defining polypharmacy using TC1 class is not accurate enough in terms of associations with health problems compared to defining polypharmacy using TC1S1 drug class, Active ingredients, OR drugs.

Table 4.35: Summery of Top 30 TC1 class pairs and their richness with respect to occurrence and ER.

\begin{tabular}{|l|c|c|}
\hline TC1 class pairs & Richness-occ & Richness-ERocc \\
\hline Anti_infectives--Central nervous system agents & 2.17 & 2.11 \\
\hline Cardiovascular agents-- Metabolic agents & 17.07 & 19.89 \\
\hline Central nervous system agents--Psychotherapeutic agents & 7.08 & 6.44 \\
\hline Central nervous system agents--Cardiovascular agents & 1.19 & 1.41 \\
\hline Anti_infectives--Respiratory agents & 2.93 & 3.16 \\
\hline Central nervous system agents--Hormones/hormone modifiers & 1.29 & 1.76 \\
\hline
\end{tabular}




\begin{tabular}{|l|l|l|}
\hline Central nervous system agents--Gastrointestinal agents & 3.32 & 3.51 \\
\hline Central nervous system agents--Respiratory agents & 1.55 & 1.51 \\
\hline Anti_infectives--Topical agents & 2.71 & 2.48 \\
\hline Cardiovascular agents--Central nervous system agents & 0.99 & 0.92 \\
\hline Central nervous system agents--Topical agents & 1.31 & 1.26 \\
\hline Central nervous system agents-- Metabolic agents & 1.57 & 1.59 \\
\hline Hormones/hormone modifiers--Respiratory agents & 1.98 & 4.16 \\
\hline Respiratory agents--Topical agents & 3.79 & 4.04 \\
\hline Anti_infectives--Hormones/hormone modifiers & 1.21 & 1.64 \\
\hline Central nervous system agents--Nutritional products & 2.08 & 2.09 \\
\hline Cardiovascular agents--Gastrointestinal agents & 3.98 & 4.61 \\
\hline Anti_infectives--Cardiovascular agents & 0.67 & 0.76 \\
\hline Cardiovascular agents--Hormones/hormone modifiers & 1.87 & 2.00 \\
\hline Cardiovascular agents--Psychotherapeutic agents & 2.73 & 3.10 \\
\hline Cardiovascular agents--Nutritional products & 2.72 & 3.73 \\
\hline Cardiovascular agents--Topical agents & 1.21 & 1.56 \\
\hline Hormones/hormone modifiers--Topical agents & 1.46 & 1.94 \\
\hline Cardiovascular agents--Respiratory agents & 0.97 & 1.11 \\
\hline Anti_infectives-- Metabolic agents & 0.58 & 0.86 \\
\hline Anti_infectives--Nutritional products & 1.21 & 1.32 \\
\hline Anti_infectives--Gastrointestinal agents & 1.24 & 1.21 \\
\hline Hormones/hormone modifiers--Psychotherapeutic agents & 2.60 & 2.73 \\
\hline Hormones/hormone modifiers--Nutritional products & 1.24 & 3.04 \\
\hline Gastrointestinal agents--Nutritional products & 3.33 & 6.23 \\
\hline
\end{tabular}

Finally, we apply the same richness analysis for TC1S1 class pairs. Table 4.36 shows the top TC1S1 class -pairs and their co-occurrences probability ranked by ER total and Table 4.37 shows the individual and joint probability of occurrence for the top 30 two-TC1S1 class polypharmacy (sorted by ER total) with richness of occurrence and ER. 
Table 4.36: Top 30 TC1S1 class-pairs and their co-occurrences probability ranked by ER total.

\begin{tabular}{|c|c|c|c|c|c|}
\hline & TC1S1 class pairs & \#occ & \#ER & \#ER/\#Occ & $P(t w o-o c c)$ \\
\hline 1 & ANALGESICS--MUSCLE_RELAXANTS & 921 & 525 & 0.57 & 0.0225 \\
\hline 2 & ANALGESICS--PENICILLINS & 1,169 & 519 & 0.44 & 0.0285 \\
\hline 3 & ANALGESICS--MACROLIDE_DERIVATIVES & 609 & 335 & 0.55 & 0.0149 \\
\hline 4 & $\begin{array}{l}\text { ANALGESICS-- } \\
\text { ANTIEMETIC/ANTIVERTIGO_AGENTS }\end{array}$ & 176 & 208 & 1.18 & 0.0043 \\
\hline 5 & $\begin{array}{l}\text { ADRENAL_CORTICAL_STEROIDS-- } \\
\text { BRONCHODILATORS }\end{array}$ & 319 & 192 & 0.60 & 0.0078 \\
\hline 6 & ANALGESICS--ANTICONVULSANTS & 281 & 162 & 0.58 & 0.0069 \\
\hline 7 & ANALGESICS--DERMATOLOGICAL_AGENTS & 322 & 160 & 0.50 & 0.0079 \\
\hline 8 & ANALGESICS--CEPHALOSPORINS & 223 & 123 & 0.55 & 0.0054 \\
\hline 9 & ANALGESICS--PROTON_PUMP_INHIBITORS & 260 & 114 & 0.44 & 0.0063 \\
\hline 10 & ANALGESICS--IRON_PRODUCTS & 157 & 107 & 0.68 & 0.0038 \\
\hline 11 & ANALGESICS--MISCELLANEOUS_ANTIBIOTICS & 114 & 102 & 0.89 & 0.0028 \\
\hline 12 & ANALGESICS--SEX_HORMONES & 278 & 101 & 0.36 & 0.0068 \\
\hline 13 & BRONCHODILATORS--PENICILLINS & 210 & 100 & 0.48 & 0.0051 \\
\hline 14 & $\begin{array}{l}\text { BRONCHODILATORS-- } \\
\text { RESPIRATORY_INHALANT_PRODUCTS }\end{array}$ & 308 & 93 & 0.30 & 0.0075 \\
\hline 15 & ANALGESICS--BRONCHODILATORS & 149 & 90 & 0.60 & 0.0036 \\
\hline 16 & $\begin{array}{l}\text { ANTIEMETIC/ANTIVERTIGO_AGENTS-- } \\
\text { ANALGESICS }\end{array}$ & 89 & 88 & 0.99 & 0.0022 \\
\hline 17 & ANALGESICS--ANTIDEPRESSANTS & 200 & 88 & 0.44 & 0.0049 \\
\hline 18 & ANALGESICS--QUINOLONES & 96 & 82 & 0.85 & 0.0023 \\
\hline 19 & MACROLIDE_DERIVATIVES--PENICILLINS & 148 & 80 & 0.54 & 0.0036 \\
\hline 20 & ADRENAL_CORTICAL_STEROIDS--ANALGESICS & 80 & 79 & 0.99 & 0.0020 \\
\hline
\end{tabular}




\begin{tabular}{|c|l|c|c|c|c|}
\hline 21 & ANTIDEPRESSANTS--ANTICONVULSANTS & 192 & 76 & 0.40 & 0.0047 \\
\hline 22 & ANALGESICS--LINCOMYCIN_DERIVATIVES & 100 & 75 & 0.75 & 0.0024 \\
\hline 23 & ANTICONVULSANTS--ANALGESICS & 165 & 73 & 0.44 & 0.0040 \\
\hline 24 & ANALGESICS-LLAXATIVES & 142 & 70 & 0.49 & 0.0035 \\
\hline 25 & $\begin{array}{l}\text { ANALGESICS-- } \\
\text { ANTIHYPERTENSIVE_COMBINATIONS }\end{array}$ & 120 & 67 & 0.56 & 0.0029 \\
\hline 26 & ANALGESICS--DIURETICS & 101 & 66 & 0.65 & 0.0025 \\
\hline 27 & $\begin{array}{l}\text { ANALGESICS-- } \\
\text { BETA_ADRENERGIC_BLOCKING_AGENTS }\end{array}$ & 138 & 65 & 0.47 & 0.0034 \\
\hline 28 & $\begin{array}{l}\text { BRONCHODILATORS-- } \\
\text { LEUKOTRIENE_MODIFIERS }\end{array}$ & 263 & 62 & 0.24 & 0.0064 \\
\hline 29 & $\begin{array}{l}\text { BRONCHODILATORS-- } \\
\text { MACROLIDE_DERIVATIVES }\end{array}$ & 175 & 62 & 0.35 & 0.0043 \\
\hline 30 & \begin{tabular}{l} 
ANALGESICS--ADRENAL_CORTICAL_STEROIDS \\
\hline
\end{tabular} & 99 & 62 & 0.63 & 0.0024 \\
\hline
\end{tabular}

Table 4.37: Individual and joint probability of occurrence for the top 30 two-TC1S1 class polypharmacy (sorted by ER total) with richness of occurrence and ER.

\begin{tabular}{|c|c|c|c|c|c|c|c|c|c|c|c|c|}
\hline & TC1S1 class pairs & $\begin{array}{l}\text { part1- } \\
\text { occ }\end{array}$ & $\begin{array}{l}\mathrm{p}(\text { part } \\
1-\mathrm{occ})\end{array}$ & $\begin{array}{l}\text { part2- } \\
\text { occ }\end{array}$ & $\begin{array}{c}\text { p(part2 } \\
\text {-occ) }\end{array}$ & $\begin{array}{l}\text { Occ-R } \\
*\end{array}$ & $\begin{array}{l}\text { part1- } \\
\text { ERocc }\end{array}$ & $\begin{array}{l}\text { p(part1- } \\
\text { ERocc }\end{array}$ & $\begin{array}{l}\text { part2- } \\
\text { ERocc }\end{array}$ & $\begin{array}{l}\text { p(part2- } \\
\text { ERocc) }\end{array}$ & $\begin{array}{l}\text { p(two- } \\
\text { case- } \\
\text { ER) }\end{array}$ & $\begin{array}{c}\text { ER-R } \\
* *\end{array}$ \\
\hline 1 & $\begin{array}{l}\text { ANALGESICS-- } \\
\text { MUSCLE_RELAXANTS }\end{array}$ & 8519 & 0.12 & 441 & 0.01 & 30.89 & 3973 & 0.2270 & 166 & 0.0095 & 0.0426 & 19.7 \\
\hline 2 & ANALGESICS--PENICILLINS & 8519 & 0.12 & 5593 & 0.08 & 3.09 & 3973 & 0.2270 & 1591 & 0.0909 & 0.0421 & 2.04 \\
\hline 3 & $\begin{array}{l}\text { ANALGESICS-- } \\
\text { MACROLIDE DERIVATIVES }\end{array}$ & 8519 & 0.12 & 3357 & 0.05 & 2.68 & 3973 & 0.2270 & 932 & 0.0532 & 0.0272 & 2.25 \\
\hline 4 & $\begin{array}{l}\text { ANALGESICS-- } \\
\text { ANTIEMETIC/ANTIVERTIGO_AGENTS }\end{array}$ & 8519 & 0.12 & 724 & 0.01 & 3.60 & 3973 & 0.2270 & 420 & 0.0240 & 0.0169 & 3.10 \\
\hline 5 & $\begin{array}{l}\text { ADRENAL_CORTICAL_STEROIDS-- } \\
\text { BRONCHODILATORS }\end{array}$ & 781 & 0.01 & 2683 & 0.04 & 19.18 & 302 & 0.0173 & 723 & 0.0413 & 0.0156 & 21.8 \\
\hline 6 & $\begin{array}{l}\text { ANALGESICS- } \\
\text { ANTICONVULSANTS }\end{array}$ & 8519 & 0.12 & 1280 & 0.02 & 3.25 & 3973 & 0.2270 & 505 & 0.0289 & 0.0131 & 2.00 \\
\hline 7 & $\begin{array}{l}\text { ANALGESICS-- } \\
\text { DERMATOLOGICAL_AGENTS }\end{array}$ & 8519 & 0.12 & 3326 & 0.05 & 1.43 & 3973 & 0.2270 & 707 & 0.0404 & 0.0130 & 1.41 \\
\hline 8 & $\begin{array}{l}\text { ANALGESICS-- } \\
\text { CEPHALOSPORINS }\end{array}$ & 8519 & 0.12 & 871 & 0.01 & 3.79 & 3973 & 0.2270 & 290 & 0.0166 & 0.0100 & 2.65 \\
\hline 9 & $\begin{array}{l}\text { ANALGESICS-- } \\
\text { PROTON_PUMP_INHIBITORS }\end{array}$ & 8519 & 0.12 & 1429 & 0.02 & 2.69 & 3973 & 0.2270 & 315 & 0.0180 & 0.0092 & 2.26 \\
\hline 10 & $\begin{array}{l}\text { ANALGESICS -- } \\
\text { IRON PRODUCTS }\end{array}$ & 8519 & 0.12 & 1013 & 0.01 & 2.29 & 3973 & 0.2270 & 394 & 0.0225 & 0.0087 & 1.70 \\
\hline 11 & $\begin{array}{l}\text { ANALGESICS-- } \\
\text { MISCELLANEOUS ANTIBIOTICS }\end{array}$ & 8519 & 0.12 & 544 & 0.01 & 3.10 & 3973 & 0.2270 & 203 & 0.0116 & 0.0083 & 3.14 \\
\hline
\end{tabular}




\begin{tabular}{|c|c|c|c|c|c|c|c|c|c|c|c|c|}
\hline 12 & $\begin{array}{l}\text { ANALGESICS-- } \\
\text { SEX_HORMONES }\end{array}$ & 8519 & 0.12 & 4945 & 0.07 & 0.83 & 3973 & 0.2270 & 559 & 0.0319 & 0.0082 & 1.13 \\
\hline 13 & $\begin{array}{l}\text { BRONCHODILATORS-- } \\
\text { PENICILLINS }\end{array}$ & 2683 & 0.04 & 5593 & 0.08 & 1.76 & 723 & 0.0413 & 1591 & 0.0909 & 0.0081 & 2.16 \\
\hline 14 & $\begin{array}{l}\text { BRONCHODILATORS-- } \\
\text { RESPIRATORY INHALANT PRODUCTS }\end{array}$ & 2683 & 0.04 & 200 & 0.00 & 72.33 & 723 & 0.0413 & 63 & 0.0036 & 0.0075 & 50.7 \\
\hline 15 & $\begin{array}{l}\text { ANALGESICS-- } \\
\text { BRONCHODILATORS }\end{array}$ & 8519 & 0.12 & 2683 & 0.04 & 0.82 & 3973 & 0.2270 & 723 & 0.0413 & 0.0073 & 0.78 \\
\hline 16 & $\begin{array}{l}\text { ANALGESICS-- } \\
\text { ANTIDEPRESSANTS }\end{array}$ & 8519 & 0.12 & 2798 & 0.04 & 1.06 & 3973 & 0.2270 & 575 & 0.0329 & 0.0071 & 0.96 \\
\hline 17 & $\begin{array}{l}\text { ANTIEMETIC/ANTIVERTIGO_AGENTS-- } \\
\text { ANALGESICS }\end{array}$ & 724 & 0.01 & 8519 & 0.12 & 1.82 & 420 & 0.0240 & 3973 & 0.2270 & 0.0071 & 1.31 \\
\hline 18 & ANALGESICS--QUINOLONES & 8519 & 0.12 & 393 & 0.01 & 3.61 & 3973 & 0.2270 & 141 & 0.0081 & 0.0066 & 3.63 \\
\hline 19 & $\begin{array}{l}\text { MACROLIDE_DERIVATIVES-- } \\
\text { PENICILLINS }\end{array}$ & 3357 & 0.05 & 5593 & 0.08 & 0.99 & 932 & 0.0532 & 1591 & 0.0909 & 0.0065 & 1.34 \\
\hline 20 & $\begin{array}{l}\text { ADRENAL_CORTICAL_STEROIDS-- } \\
\text { ANALGESICS }\end{array}$ & 781 & 0.01 & 8519 & 0.12 & 1.52 & 302 & 0.0173 & 3973 & 0.2270 & 0.0064 & 1.63 \\
\hline 21 & $\begin{array}{l}\text { ANTIDEPRESSANTS-- } \\
\text { ANTICONVULSANTS }\end{array}$ & 2798 & 0.04 & 1280 & 0.02 & 6.76 & 575 & 0.0329 & 505 & 0.0289 & 0.0062 & 6.50 \\
\hline 22 & $\begin{array}{l}\text { ANALGESICS-- } \\
\text { LINCOMYCIN_DERIVATIVES }\end{array}$ & 8519 & 0.12 & 178 & 0.00 & 8.31 & 3973 & 0.2270 & 64 & 0.0037 & 0.0061 & 7.32 \\
\hline 23 & $\begin{array}{l}\text { ANTICONVULSANTS-- } \\
\text { ANALGESICS }\end{array}$ & 1280 & 0.02 & 8519 & 0.12 & 1.91 & 505 & 0.0289 & 3973 & 0.2270 & 0.0059 & 0.90 \\
\hline 24 & $\begin{array}{l}\text { ANALGESICS-- } \\
\text { LAXATIVES }\end{array}$ & 8519 & 0.12 & 268 & 0.00 & 7.84 & 3973 & 0.2270 & 91 & 0.0052 & 0.0057 & 4.81 \\
\hline 25 & $\begin{array}{l}\text { ANALGESICS-- } \\
\text { ANTIHYPERTENSIVE_COMBINATIONS }\end{array}$ & 8519 & 0.12 & 1925 & 0.03 & 0.92 & 3973 & 0.2270 & 242 & 0.0138 & 0.0054 & 1.73 \\
\hline 26 & $\begin{array}{l}\text { ANALGESICS-- } \\
\text { DIURETICS }\end{array}$ & 8519 & 0.12 & 716 & 0.01 & 2.09 & 3973 & 0.2270 & 164 & 0.0094 & 0.0053 & 2.52 \\
\hline 27 & $\begin{array}{l}\text { ANALGESICS--BETA-- } \\
\text { ADRENERGIC BLOCKING AGENTS }\end{array}$ & 8519 & 0.12 & 0 & 0.00 & 0 & 3973 & 0.2270 & 0 & 0 & 0.0053 & 0 \\
\hline 28 & $\begin{array}{l}\text { ANALGESICS-- } \\
\text { ADRENAL CORTICAL STEROIDS }\end{array}$ & 8519 & 0.12 & 781 & 0.01 & 1.88 & 3973 & 0.2270 & 302 & 0.0173 & 0.0050 & 1.28 \\
\hline 29 & $\begin{array}{l}\text { BRONCHODILATORS-- } \\
\text { LEUKOTRIENE_MODIFIERS }\end{array}$ & 2683 & 0.04 & 457 & 0.01 & 27.03 & 723 & 0.0413 & 97 & 0.0055 & 0.0050 & 21.9 \\
\hline 30 & $\begin{array}{l}\text { BRONCHODILATORS-- } \\
\text { MACROLIDE_DERIVATIVES }\end{array}$ & 2683 & 0.04 & 3357 & 0.05 & 2.45 & 723 & 0.0413 & 932 & 0.0532 & 0.0050 & 2.28 \\
\hline
\end{tabular}

* Occ-R here is occurrence Richness

** ER-R here is ER Richness

The pair ANTIHYPERTENSIVE_COMBINATIONS -- CALCIUM_CHANNEL_BLOCKING_AGENTS, which is Ranked 47 by ER visits, have much higher ER richness compared to its occurrence richness (16.44739 vs. 44.10178). On the other hand, the pair ANALGESICS --MUSCLE_RELAXANTS has higher occurrence richness and lower ER richness (30.89 vs. 19.77). This could mean that the pair ANTIHYPERTENSIVE_COMBINATIONS --CALCIUM_CHANNEL_BLOCKING_AGENTS jointly may be less safety than ANALGESICS -- MUSCLE_RELAXANTS even though the later occurred more frequently.

Figure 4.16 shows the richness of occurrence and the richness of ER visits values for TC1S1 drug class pairs and Table $\mathbf{4 . 3 8}$ shows example pairs for TC1S1 classes with high occurrence richness and low ER richness, high occurrence richness and low ER richness, and low occurrence richness and high ER richness. Based on the richness results, only one TC1S1 class pair was found for the case (low occurrence richness and high ER richness). 


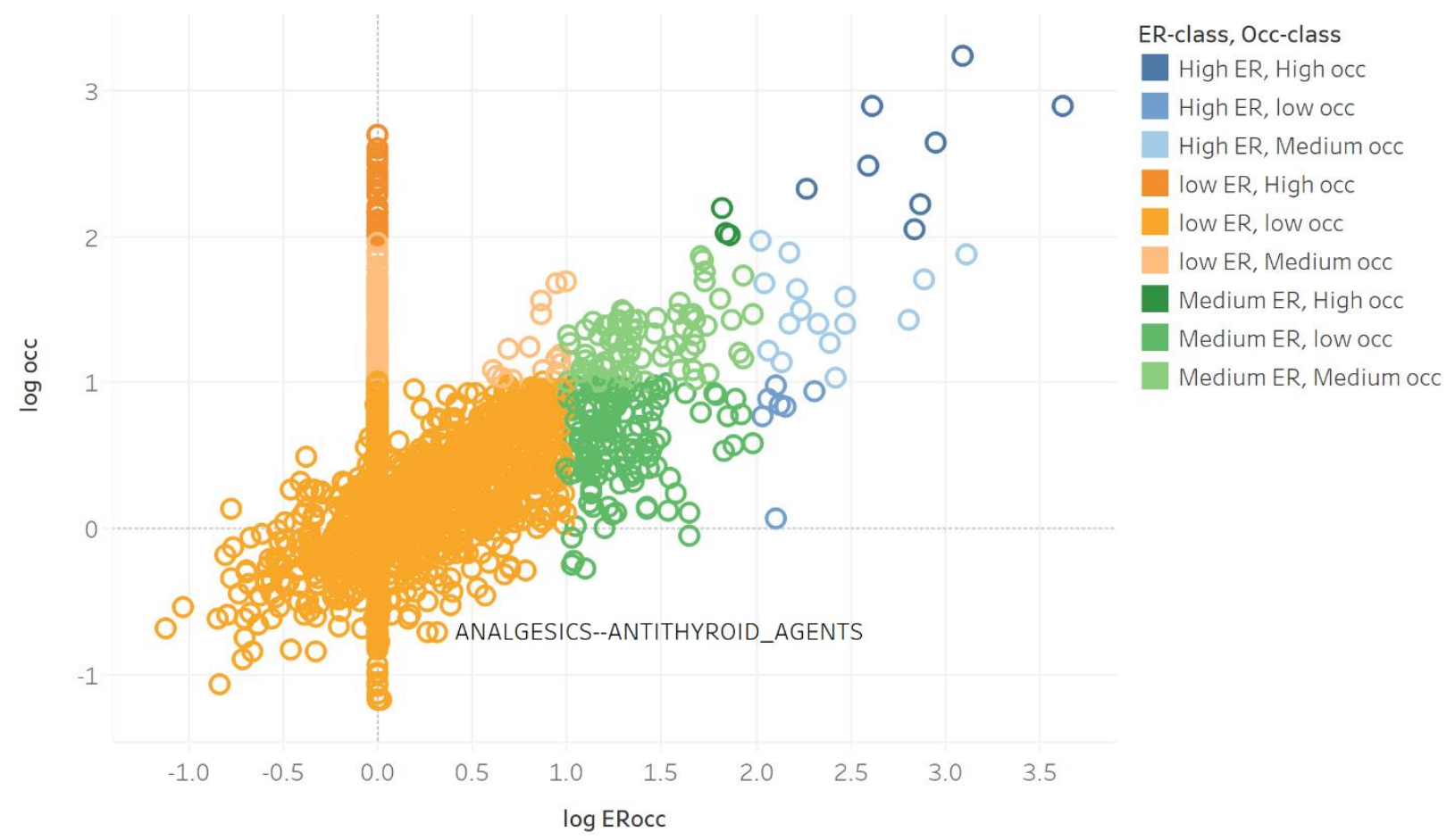

Figure 4.16 Relationship between TC1S1 class pairs occurrence and ER richness. Logarithms are in base 10 . 
Table 4.38 Example of TC1S1 class pairs with high occurrence richness and low ER richness, high occurrence richness and low ER richness, and low occurrence richness and high ER richness.

\begin{tabular}{|c|c|c|c|}
\hline & TC1S1 class pairs & $\begin{array}{l}\text { ER- } \\
\text { richness }\end{array}$ & $\begin{array}{c}\text { Occ- } \\
\text { Richness }\end{array}$ \\
\hline \multirow{5}{*}{$\begin{array}{l}\text { high occurrence } \\
\text { richness and } \\
\text { high ER richness }\end{array}$} & $\begin{array}{l}\text { MISCELLANEOUS_GENITOURINARY_TRACT_AGENTS-- } \\
\text { QUINOLONES }\end{array}$ & 387.42 & 306.71 \\
\hline & ANTIDIURETIC_HORMONES--DIGESTIVE_ENZYMES & $4,138.36$ & 787.61 \\
\hline & ANTIRHEUMATICS--ANTIMETABOLITES & 886.79 & 438.32 \\
\hline & $\begin{array}{l}\text { CHOLINESTERASE_INHIBITORS-- } \\
\text { MISCELLANEOUS_CENTRAL_NERVOUS_SYSTEM_AGENT }\end{array}$ & 413.83 & 795.56 \\
\hline & $\begin{array}{l}\text { MISCELLANEOUS_CARDIOVASCULAR_AGENTS-- } \\
\text { MISCELLANEOUS_CENTRAL_NERVOUS_SYSTEM_AGENT }\end{array}$ & $1,241.51$ & $1,750.24$ \\
\hline \multirow{10}{*}{$\begin{array}{l}\text { high occurrence } \\
\text { richness and } \\
\text { low ER richness }\end{array}$} & ANTICOAGULANTS--CALCITONIN & 0 & 492.25 \\
\hline & (5_AMINOSALICYLATES)--ANTIMETABOLITES & 0 & 345.25 \\
\hline & $\begin{array}{l}\text { ADRENAL_CORTICAL_STEROIDS-- } \\
\text { CHOLINERGIC_AGONISTS }\end{array}$ & 0 & 161.35 \\
\hline & $\begin{array}{l}\text { ALKYLATING_AGENTS-- } \\
\text { ANTIADRENERGIC_AGENTSPERIPHERALLY_ACTING }\end{array}$ & 0 & 282.55 \\
\hline & AMEBICIDES--ANTHELMINTICS & 0 & 203.25 \\
\hline & ANTIANGINAL_AGENTS--VASODILATORS & 0 & 400.05 \\
\hline & ANTIARRHYTHMIC_AGENTS--CALCITONIN & 0 & 316.62 \\
\hline & ANTIDIARRHEALS--ANTIRHEUMATICS & 0 & 228.29 \\
\hline & $\begin{array}{l}\text { ANTIHYPERURICEMIC_AGENTS-- } \\
\text { CHOLINESTERASE_INHIBITORS }\end{array}$ & 0 & 238.67 \\
\hline & ANTIMALARIAL_AGENTS--CHOLINERGIC_AGONISTS & 0 & 369.55 \\
\hline $\begin{array}{l}\text { low occurrence } \\
\text { richness and } \\
\text { high ER richness }\end{array}$ & NASAL_PREPARATIONS--URINARY_ANTISPASMODICS & 126.55 & 1.15 \\
\hline
\end{tabular}


It is important to observe and compare how polypharmacy of TC1 drug class and polypharmacy of TC1S1 drug class flag and highlight risks. This helps people in health care fields to know which one is more reliable at certain health conditions. Thus, we compare the results obtained from the associations between both drug classes and ER visits. This involves their ranking based on ER visits, and their richness with respect to ER occurrence. Table 4.39 shows show few examples of single TC1 drug classes along with their original ranking based on ER visits for patients, some of TC1S1 pairs which individually form subclasses from the parent TC1 class as well as their original ranking based on the ER visits, and finally, the richness measure for TC1S1 class pairs.

From Table 4.39 we can observe that the single TC1 classes Respiratory agents and Antiinfectives are ranked by ER visits as 4 and 2 respectively. This means that these drug classes are associated with high risk according to the definition of TC1 drug class. However, if we consider the TC1S1 pairs, we can notice that the pair BRONCHODILATORS--RESPIRATORY_INHALANT_PRODUCTS has high ER richness while the pair ANTIHISTAMINES--BRONCHODILATORS has low ER richness even though they both are formed by combining TC1S1 subclasses (i.e., BRONCHODILATORS, RESPIRATORY_INHALANT_PRODUCTS, ANTIHISTAMINES, and BRONCHODILATORS) that belong to the same super TC1 class (Respiratory agents). The high richness for the pair BRONCHODILATORS-RESPIRATORY_INHALANT_PRODUCTS means that these drug classes together tend to have more association with ER than what is expected from the occurrence of individual subclasses. On the other hand, the low richness for the pair ANTIHISTAMINES-BRONCHODILATORS mean that these drug classes together tend to have much lower association with ER and considered to be relatively much safer. As a consequence, we can conclude here that even though TC1 single class (Respiratory agents) is flagged to be risky based on ER visits, the richness for its subclasses pairs indicates that the subclasses may not necessarily be at the same level of risk. The same argument apply for the case of (Anti-infectives) and the corresponding TC1S1 pairs:

\section{- ANTITUBERCULOSIS_AGENTS--LINCOMYCIN_DERIVATIVES}

- ANTIFUNGALS--LINCOMYCIN_DERIVATIVES

- ANTIMALARIAL_AGENTS--ANTIVIRAL_AGENTS

The reason behind this different estimates of risks is because TC1 drug classes are broad, general, and modeled classes compared to those for TC1S1. As a result, we can now infer that the TC1S1 approach to defining polypharmacy is more informative and provide a more detailed view of looking at more branched drug classes and their associations with health problems. Table 4.41 shows the above Two TC1 classes along with their TC1S1 subclasses. 
Table 4.39 Comparison of some single TC1 classes and some of their continent TC1S1 class pairs ranking based on ER visits along with the richness of the TC1S1 class pairs.

\begin{tabular}{|c|c|c|c|c|}
\hline Single TC1 class & \#ER/\#Occ & Multiple TC1S1 & \#ER/\#Occ & $\begin{array}{l}\text { ER- } \\
\text { Richness }\end{array}$ \\
\hline \multirow{2}{*}{$\begin{array}{l}\text { Respiratory agents ranked } 4 \\
\text { by ER. }\end{array}$} & \multirow{2}{*}{0.25} & $\begin{array}{l}\text { BRONCHODILATORS-- } \\
\text { RESPIRATORY_INHALANT_PRODUCTS } \\
\text { (Ranked } 16 \text { by ER) }\end{array}$ & 0.30 & 72.33 \\
\hline & & $\begin{array}{l}\text { ANTIHISTAMINES-- } \\
\text { BRONCHODILATORS (ranked } 67 \text { by } \\
\text { ER) }\end{array}$ & 0.28 & 7.32 \\
\hline \multirow{3}{*}{$\begin{array}{c}\text { Anti-infectives ranked } 2 \text { by } \\
\text { ER. }\end{array}$} & \multirow{3}{*}{0.3} & $\begin{array}{l}\text { ANTITUBERCULOSIS_AGENTS-- } \\
\text { LINCOMYCIN_DERIVATIVES } \\
865\end{array}$ & 1 & 775.94 \\
\hline & & $\begin{array}{l}\text { ANTIFUNGALS-- } \\
\text { LINCOMYCIN_DERIVATIVES } \\
337\end{array}$ & 1 & 14.10 \\
\hline & & $\begin{array}{l}\text { ANTIMALARIAL_AGENTS-- } \\
\text { ANTIVIRAL_AGENTS } \\
714\end{array}$ & 0.5 & 3.30 \\
\hline
\end{tabular}

Table 4.40 Two TC1 classes and their TC1S1 subclasses.

\begin{tabular}{|l|l|}
\hline TC1 drug class Name & TC1S1 drug class Name \\
\hline Respiratory agents & $\begin{array}{l}\text { antihistamines } \\
\text { antitussives } \\
\text { bronchodilators }\end{array}$ \\
& $\begin{array}{l}\text { decongestants } \\
\text { expectorants } \\
\text { miscellaneous respiratory agents } \\
\text { respiratory inhalant products } \\
\text { antiasthmatic combinations } \\
\text { upper respiratory combinations } \\
\text { leukotriene modifiers } \\
\text { lung surfactants } \\
\end{array}$ \\
\hline
\end{tabular}




\begin{tabular}{|c|c|}
\hline Anti-infectives & $\begin{array}{l}\text { amebicides } \\
\text { anthelmintics } \\
\text { antifungals } \\
\text { antimalarial agents } \\
\text { antituberculosis agents } \\
\text { antiviral agents } \\
\text { carbapenems } \\
\text { cephalosporins } \\
\text { leprostatics } \\
\text { macrolide derivatives } \\
\text { miscellaneous antibiotics } \\
\text { penicillins } \\
\text { quinolones } \\
\text { sulfonamides } \\
\text { tetracyclines } \\
\text { urinary anti-infectives } \\
\text { aminoglycosides } \\
\text { glycylcyclines }\end{array}$ \\
\hline
\end{tabular}

Now, we will compare the estimate of risks for TC1 drug class and TC1S1 drug class for the case whereby the two TC1S1 classes forming a pair are originated from different two TC1 classes. Table 4.41 shows three examples of TC1 drug class pairs along with their original ranking based on ER visits for patients and their ER richness and some of TC1S1 pairs which individually form subclasses from the two parent TC1 classes as well as their original ranking based on the ER visits, and finally, the richness measure for TC1S1 class pairs. It can be observed that even though the TC1 class pairs (antineoplastics--miscellaneous agents), (cardiovascular agents--coagulation modifiers), and (central nervous system agents-- miscellaneous agents) are ranked relatively low based on ER visits sort (92, 44, and 33, respectively), and have low ER richness using TC1 definition, they have high ER richness using TC1S1 definition. This indicates that a single TC1 drug class final ranking and richness could be affected by the results coming from so many subclasses embedded under this class, and so this emphasizes that using this type of drug classes may not 
be an appropriate choice when we want an accurate, reliable, and more specific associations with health problems.

Table 4.41 Comparison of some TC1 class pairs and some of their continent TC1S1 class pairs ranking based on ER visits along with their richness values.

\begin{tabular}{|c|c|c|c|c|c|}
\hline $\begin{array}{l}\text { Parent TC1 } \\
\text { pair }\end{array}$ & \#ER/\#Occ & $\begin{array}{l}\text { ER- } \\
\text { Richness }\end{array}$ & TC1S1 pair & \#ER/\#Occ & $\begin{array}{l}\text { ER- } \\
\text { Richness }\end{array}$ \\
\hline $\begin{array}{l}\text { antineoplastics } \\
-- \\
\text { miscellaneous } \\
\text { agents } \\
\text { (ranked } 92 \text { by } \\
\text { ER) }\end{array}$ & 1 & 16.26 & $\begin{array}{l}\text { ANTIRHEUMATICS-- } \\
\text { ANTIMETABOLITES } \\
\text { Ranked } 154 \text { By ER }\end{array}$ & 0.16 & 886.79 \\
\hline $\begin{array}{l}\text { cardiovascular } \\
\text { agents } \\
-- \\
\text { coagulation } \\
\text { modifiers } \\
\text { (ranked } 44 \text { by } \\
\text { ER) }\end{array}$ & 0.3 & 6.68 & $\begin{array}{c}\text { ANTICOAGULANTS- } \\
\text { VASODILATORS } \\
156\end{array}$ & 0.6 & 730.3 \\
\hline $\begin{array}{l}\text { central } \\
\text { nervous } \\
\text { system agents } \\
\text {-- } \\
\text { miscellaneous } \\
\text { agents } \\
\text { (ranked } 33 \text { by } \\
\text { ER) }\end{array}$ & 0.37 & 3.42 & $\begin{array}{c}\text { CHOLINESTERASE INHIBITORS- } \\
\text {-URINARY_ANTISPASMODICS }\end{array}$ & 0.6 & 689.72 \\
\hline
\end{tabular}


Table 4.42 summarizes top 20 ER richness ranked by the corresponding ER visits for patients to provide a comparative overview of the richness values for each definition.

Table 4.42: Summary of ER richness occurrence for the top 20 polypharmacy using each definition ranked by \#ER visits.

\begin{tabular}{|c|c|c|c|c|c|c|c|}
\hline Drug pairs & Drugs & Active ingredient pairs & Al & TC1 class Pairs & TC1 & TC1S1 class Pairs & TC1S1 \\
\hline $\begin{array}{l}\text { HYDROCO/APAP-- } \\
\text { IBUPROFEN }\end{array}$ & 13.15 & $\begin{array}{l}\text { ACETAMINOPHEN-- } \\
\text { HYDROCODONE } \\
\text { BITARTRATE }\end{array}$ & 6133.93 & $\begin{array}{l}\text { Anti_infectives--Central } \\
\text { nervous system agents }\end{array}$ & 2.11 & $\begin{array}{l}\text { ANALGESICS-- } \\
\text { MUSCLE_RELAXANTS }\end{array}$ & 19.77 \\
\hline $\begin{array}{l}\text { AMOXICILLIN-- } \\
\text { IBUPROFEN }\end{array}$ & 8.62 & $\begin{array}{l}\text { ACETAMINOPHEN-- } \\
\text { CODEINE PHOSPHATE }\end{array}$ & 0 & $\begin{array}{l}\text { Cardiovascular agents-- } \\
\text { Metabolic agents }\end{array}$ & 19.89 & ANALGESICS--PENICILLINS & 2.04 \\
\hline $\begin{array}{l}\text { AMOXICILLIN-- } \\
\text { HYDROCO/APAP }\end{array}$ & 7.77 & $\begin{array}{l}\text { AMOXICILLIN-- } \\
\text { CLAVULANATE } \\
\text { POTASSIUM }\end{array}$ & 0 & $\begin{array}{l}\text { Central nervous system } \\
\text { agents-- } \\
\text { Psychotherapeutic } \\
\text { agents }\end{array}$ & 6.44 & $\begin{array}{l}\text { ANALGESICS-- } \\
\text { MACROLIDE_DERIVATIVES }\end{array}$ & 2.25 \\
\hline $\begin{array}{l}\text { APAP/CODEINE-- } \\
\text { AZITHROMYCIN }\end{array}$ & 40.44 & $\begin{array}{l}\text { ACETAMINOPHEN-- } \\
\text { OXYCODONE } \\
\text { HYDROCHLORIDE }\end{array}$ & 213.13 & $\begin{array}{l}\text { Central nervous system } \\
\text { agents--Cardiovascular } \\
\text { agents }\end{array}$ & 1.41 & $\begin{array}{l}\text { ANALGESICS-- } \\
\text { ANTIEMETIC/ANTIVERTIGO_ } \\
\text { AGENTS }\end{array}$ & 3.10 \\
\hline $\begin{array}{l}\text { ALBUTEROL-- } \\
\text { AMOXICILLIN }\end{array}$ & 1.55 & $\begin{array}{l}\text { SULFAMETHOXAZOLE-- } \\
\text { TRIMETHOPRIM }\end{array}$ & 0 & $\begin{array}{l}\text { Anti_infectives-- } \\
\text { Respiratory agents }\end{array}$ & 3.16 & $\begin{array}{l}\text { ADRENAL_CORTICAL_STERO } \\
\text { IDS--BRONCHODILATORS }\end{array}$ & 21.83 \\
\hline $\begin{array}{l}\text { AMOXICILLIN-- } \\
\text { AZITHROMYCIN }\end{array}$ & 17.74 & $\begin{array}{l}\text { AMOXICILLIN-- } \\
\text { IBUPROFEN }\end{array}$ & 2.03 & $\begin{array}{l}\text { Central nervous system } \\
\text { agents-- } \\
\text { Hormones/hormone } \\
\text { modifiers }\end{array}$ & 1.76 & $\begin{array}{l}\text { ANALGESICS- } \\
\text { ANTICONVULSANTS }\end{array}$ & 2.00 \\
\hline $\begin{array}{l}\text { AZITHROMYCIN-- } \\
\text { HYDROCO/APAP }\end{array}$ & 4.71 & $\begin{array}{l}\text { HYDROCHLOROTHIAZID } \\
\text { E--LISINOPRIL }\end{array}$ & 54.59 & $\begin{array}{l}\text { Central nervous system } \\
\text { agents--Gastrointestinal } \\
\text { agents }\end{array}$ & 3.51 & $\begin{array}{l}\text { ANALGESICS-- } \\
\text { DERMATOLOGICAL_AGENTS }\end{array}$ & 1.41 \\
\hline $\begin{array}{l}\text { CYCLOBENZAPR-- } \\
\text { HYDROCO/APAP }\end{array}$ & 3.80 & $\begin{array}{l}\text { ALBUTEROL SULFATE-- } \\
\text { PREDNISONE }\end{array}$ & 10.90 & $\begin{array}{l}\text { Central nervous system } \\
\text { agents--Respiratory } \\
\text { agents }\end{array}$ & 1.51 & $\begin{array}{l}\text { ANALGESICS- } \\
\text { CEPHALOSPORINS }\end{array}$ & 2.65 \\
\hline $\begin{array}{l}\text { CYCLOBENZAPR-- } \\
\text { IBUPROFEN }\end{array}$ & 11.21 & $\begin{array}{l}\text { AMOXICILLIN-- } \\
\text { ALBUTEROL SULFATE }\end{array}$ & 2.32 & $\begin{array}{l}\text { Anti_infectives--Topical } \\
\text { agents }\end{array}$ & 2.48 & $\begin{array}{l}\text { ANALGESICS-- } \\
\text { PROTON_PUMP_INHIBITOR } \\
\text { S }\end{array}$ & 2.26 \\
\hline $\begin{array}{l}\text { IBUPROFEN-- } \\
\text { OXYCOD/APAP }\end{array}$ & 20.84 & $\begin{array}{l}\text { ALBUTEROL SULFATE-- } \\
\text { FLUTICASONE } \\
\text { PROPIONATE }\end{array}$ & 1.56 & $\begin{array}{l}\text { Cardiovascular agents-- } \\
\text { Central nervous system } \\
\text { agents }\end{array}$ & 0.92 & $\begin{array}{l}\text { ANALGESICS -- } \\
\text { IRON_PRODUCTS }\end{array}$ & 1.70 \\
\hline $\begin{array}{l}\text { IBUPROFEN-- } \\
\text { PREDNISONE }\end{array}$ & 8.46 & $\begin{array}{l}\text { CYCLOBENZAPRINE } \\
\text { HYDROCHLORIDE-- } \\
\text { IBUPROFEN }\end{array}$ & 20.22 & $\begin{array}{l}\text { Central nervous system } \\
\text { agents--Topical agents }\end{array}$ & 1.26 & $\begin{array}{l}\text { ANALGESICS-- } \\
\text { MISCELLANEOUS_ANTIBIOTI } \\
\text { CS }\end{array}$ & 3.14 \\
\hline $\begin{array}{l}\text { AMLODIPINE-- } \\
\text { LISINOPRIL }\end{array}$ & 11.88 & $\begin{array}{l}\text { AZITHROMYCIN } \\
\text { DIHYDRATE-- } \\
\text { IBUPROFEN }\end{array}$ & 11.12 & $\begin{array}{l}\text { Central nervous system } \\
\text { agents--M Metabolic } \\
\text { agents }\end{array}$ & 1.59 & $\begin{array}{l}\text { ANALGESICS-- } \\
\text { SEX_HORMONES }\end{array}$ & 1.13 \\
\hline $\begin{array}{l}\text { AZITHROMYCIN-- } \\
\text { IBUPROFEN }\end{array}$ & 40.44 & $\begin{array}{l}\text { IBUPROFEN-- } \\
\text { PREDNISONE }\end{array}$ & 1.28 & $\begin{array}{l}\text { Hormones/hormone } \\
\text { modifiers--Respiratory } \\
\text { agents }\end{array}$ & 4.16 & $\begin{array}{l}\text { BRONCHODILATORS-- } \\
\text { PENICILLINS }\end{array}$ & 2.16 \\
\hline
\end{tabular}




\begin{tabular}{|c|c|c|c|c|c|c|c|}
\hline $\begin{array}{l}\text { CLINDAMYCIN-- } \\
\text { HYDROCO/APAP }\end{array}$ & 1.55 & $\begin{array}{l}\text { AMLODIPINE BESYLATE- } \\
\text {-LISINOPRIL }\end{array}$ & 5.98 & $\begin{array}{l}\text { Respiratory agents-- } \\
\text { Topical agents }\end{array}$ & 4.04 & $\begin{array}{l}\text { BRONCHODILATORS-- } \\
\text { RESPIRATORY_INHALANT_P } \\
\text { RODUCTS }\end{array}$ & 50.70 \\
\hline $\begin{array}{l}\text { AZITHROMYCIN-- } \\
\text { PREDNISONE }\end{array}$ & 17.74 & $\begin{array}{l}\text { AZITHROMYCIN } \\
\text { DIHYDRATE-- } \\
\text { PREDNISONE }\end{array}$ & 28.04 & $\begin{array}{l}\text { Anti_infectives-- } \\
\text { Hormones/hormone } \\
\text { modifiers }\end{array}$ & 1.64 & $\begin{array}{l}\text { ANALGESICS-- } \\
\text { BRONCHODILATORS }\end{array}$ & 0.78 \\
\hline $\begin{array}{l}\text { APAP/CODEINE-- } \\
\text { IBUPROFEN }\end{array}$ & 4.71 & $\begin{array}{l}\text { ETHINYL ESTRADIOL-- } \\
\text { NORETHINDRONE } \\
\text { ACETATE }\end{array}$ & 0.81 & $\begin{array}{l}\text { Central nervous system } \\
\text { agents--Nutritional } \\
\text { products }\end{array}$ & 2.09 & $\begin{array}{l}\text { ANALGESICS-- } \\
\text { ANTIDEPRESSANTS }\end{array}$ & 0.96 \\
\hline $\begin{array}{l}\text { HYDROCO/APAP-- } \\
\text { PROMETHAZINE }\end{array}$ & 3.80 & $\begin{array}{l}\text { ALBUTEROL SULFATE-- } \\
\text { BUDESONIDE }\end{array}$ & 3.58 & $\begin{array}{l}\text { Cardiovascular agents-- } \\
\text { Gastrointestinal agents }\end{array}$ & 4.61 & $\begin{array}{l}\text { ANTIEMETIC/ANTIVERTIGO_ } \\
\text { AGENTS--ANALGESICS }\end{array}$ & 1.31 \\
\hline $\begin{array}{l}\text { LISINOPRIL-- } \\
\text { SIMVASTATIN }\end{array}$ & 11.21 & $\begin{array}{l}\text { CYCLOBENZAPRINE } \\
\text { HYDROCHLORIDE-- } \\
\text { NAPROXEN }\end{array}$ & 0.00 & $\begin{array}{l}\text { Anti_infectives-- } \\
\text { Cardiovascular agents }\end{array}$ & 0.76 & ANALGESICS--QUINOLONES & 3.63 \\
\hline $\begin{array}{l}\text { IBUPROFEN-- } \\
\text { TRAMADOL HCL }\end{array}$ & 20.84 & $\begin{array}{l}\text { IBUPROFEN-- } \\
\text { TRAMADOL } \\
\text { HYDROCHLORIDE }\end{array}$ & 1.01 & $\begin{array}{l}\text { Cardiovascular agents-- } \\
\text { Hormones/hormone } \\
\text { modifiers }\end{array}$ & 2.00 & $\begin{array}{l}\text { MACROLIDE_DERIVATIVES-- } \\
\text { PENICILLINS }\end{array}$ & 1.34 \\
\hline $\begin{array}{l}\text { ALBUTEROL-- } \\
\text { PREDNISONE }\end{array}$ & 13.15 & $\begin{array}{l}\text { AMOXICILLIN-- } \\
\text { AZITHROMYCIN } \\
\text { DIHYDRATE }\end{array}$ & 93.18 & $\begin{array}{l}\text { Cardiovascular agents-- } \\
\text { Psychotherapeutic } \\
\text { agents }\end{array}$ & 3.10 & $\begin{array}{l}\text { ADRENAL_CORTICAL_STERO } \\
\text { IDS--ANALGESICS }\end{array}$ & 1.63 \\
\hline
\end{tabular}

As observed from table 4.42, the polypharmacy of active ingredients seems to be more sensitive than other definitions for the richness measure. The richness of active ingredients may not necessarily align with the richness of the parent drugs. For example, the drug pair (ALBUTEROL\#AMOXICILLIN) has drug-richness that is equal to 3.79 while the active ingredient pair (AMOXICILLIN\#ALBUTEROL SULFATE) has Al- richness that is equal to 1.55. On the other hand, the richness for drug pair (AMOXICILLIN\#AZITHROMYCIN) is 1.12898, but for Al richness, it decreased to 0.50 for the pair (AMOXICILLIN\#AZITHROMYCIN DIHYDRATE). Thus, these differentiation should be taken into consideration while studying polypharmacy health problems. 


\subsection{Automated thresholds for polypharmacy}

The following sub sections provide tables and figures that shows and visualize the values of the Delta function (discussed previously in Chapter 3 ) for each polypharmacy category and discuss their results values.

\subsubsection{Delta Function}

As illustrated previously in Chapter 3, we define the Delta function as the difference in ER values between two thresholds as the following $\Delta(k, j)=\frac{\left(\frac{\# E R}{\# P P}\right)_{k}-\left(\frac{\# E R}{\# P P}\right)_{j}}{\left(\frac{\# E R}{\# P P}\right)_{j}}$, and we consider a specific case of the delta function by which we estimate the change attributed to each threshold $\mathrm{k}$ and the threshold 1 assuming that, using any polypharmacy definition, the association between single element and ER problems should be ideally the least. The percentage threshold can then be chosen by considering the variation of $\Delta(k, 1)$. A specific percentage increase of this measure can be chosen depending on the risk level that is desired to be investigated. We use $50 \%$ increase as an example here. In this section, we will use an exemplary case where we study $50 \%$ increase in the variation of $\Delta(k, 1)$ to determine the problematic thresholds for each definition. The results will be presented started by polypharmacy of drugs followed by polypharmacy of axtive ingredients followed by polypharmacy of drug classes. Table 4.43 and Figure 4.17 show the values of delta function for the drug-based polypharmacy definition, respectively. 


\begin{tabular}{|rrrrrrr|r|}
\hline k value & \multicolumn{1}{r}{ Y2011 } & Y2012 & Y2013 & Y2014 & \multicolumn{2}{c|}{ Y2015 } \\
\hline 1 & 0 & 0 & 0 & 0 & 0 \\
\hline 2 & 0.189485 & 0.222993 & 0.220109 & 0.273258 & 0.299636 \\
\hline 3 & 0.309234 & 0.345682 & 0.345899 & 0.328043 & 0.419536 \\
\hline 4 & 0.497979 & 0.46116 & 0.485189 & 0.524823 & 0.610022 \\
\hline 5 & 0.588648 & 0.615404 & 0.563554 & 0.626544 & 0.706004 \\
\hline 6 & 0.635043 & 0.779675 & 0.7715 & 0.809136 & 1.026679 \\
\hline 7 & 0.782341 & 0.902517 & 0.969403 & 0.970613 & 1.22419 \\
\hline 8 & 0.874798 & 0.968218 & 1.177683 & 1.345501 & 1.454539 \\
\hline 9 & 0.908378 & 1.10086 & 1.287882 & 1.420752 & 1.719018 \\
\hline 10 & 1.464232 & 1.116019 & 1.498891 & 1.914181 & 1.95108 \\
\hline 11 & 1.289863 & 1.609262 & 1.584395 & 1.573514 & 1.896542 \\
\hline 12 & 1.783303 & 3.380951 & 2.535669 & 2.640168 & 2.590159 \\
\hline 13 & 2.022353 & 2.608169 & 2.108939 & 1.830684 & 2.554512 \\
\hline 14 & 2.058811 & 2.140507 & 1.878488 & 2.91856 & 3.930735 \\
\hline 15 & 2.387897 & 2.936102 & 2.672032 & 2.239184 & 2.942776 \\
\hline 16 & 2.46455 & 3.267935 & 3.878675 & 4.386803 & 3.977708 \\
\hline
\end{tabular}

\section{Delta relative to $\mathrm{k}=1$ : Drug}

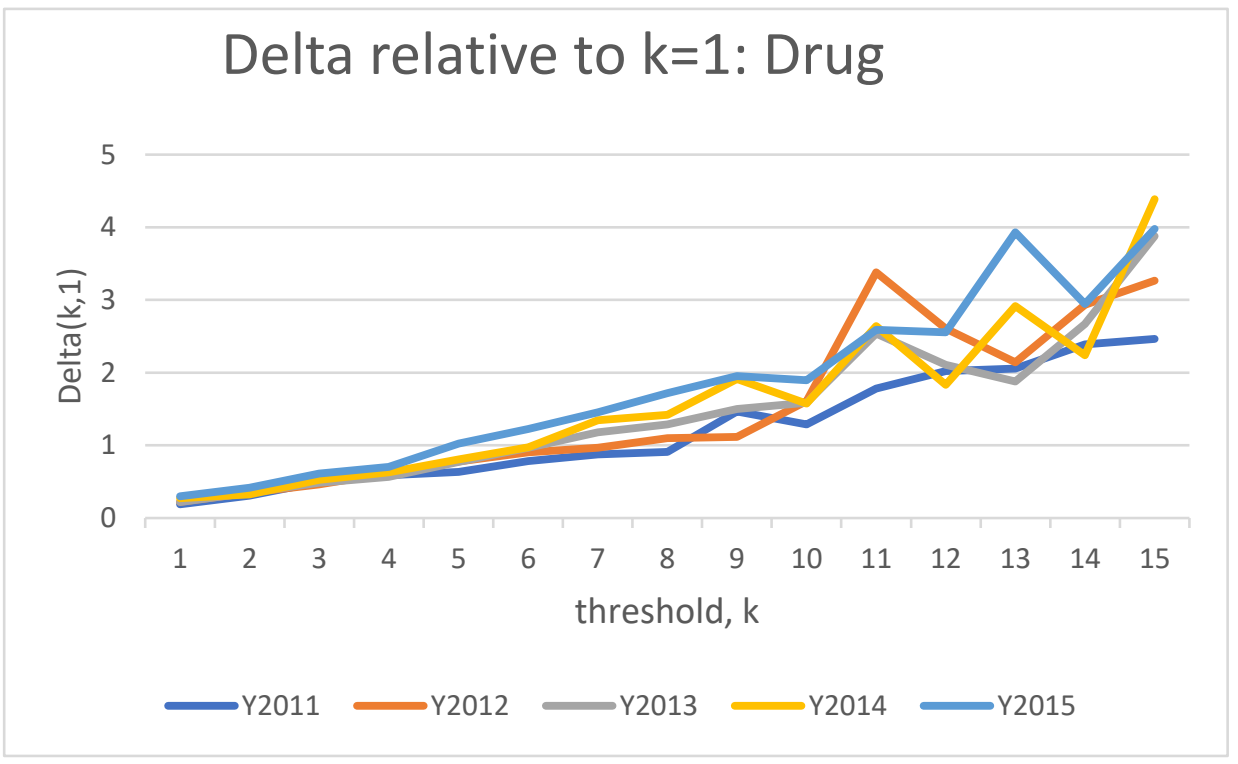

Table 4.43. Values of $\Delta(K, 1)$ for drugs PP vs. ER measure for years (2011-2015)
Figure 4.17. Values of $\Delta(\mathrm{K}, 1)$ for drug PP vs. ER measure for years (20112015)

In general, the variation $\Delta(\mathrm{K}, 1)$ for drug polypharmacy increases with increasing number of threshold. This implies that polypharmacy with higher number of drugs (i.e., greater than 1 ) is likely to be associated with more ER problems. In particular, $\Delta(K, 1)$ is less than 1 for the thresholds (1-5), ranges from 1 to around 2.5 for thresholds (6-13), and nearly about 2.6 to 3 for thresholds (14-16). Also, it can be observed from figure 4.17 that the variation $\Delta(K, 1)$ has an overall increase over time from 2011 to 2015.

Table 4.44 and figure 4.18 show the values of delta function for the polypharmacy of active ingredients. 


\begin{tabular}{|rrrrrrr|}
\hline k value & \multicolumn{1}{r}{ Y2011 } & Y2012 & \multicolumn{1}{c}{ Y2013 } & \multicolumn{2}{c|}{ Y2014 } & \multicolumn{2}{c|}{ Y2015 } \\
\hline 1 & 0 & 0 & 0 & 0 & 0 \\
\hline 2 & 0.166546 & 0.219991 & 0.156289 & 0.259154 & 0.29883 \\
\hline 3 & 0.363161 & 0.323095 & 0.39805 & 0.381356 & 0.507789 \\
\hline 4 & 0.383793 & 0.449688 & 0.416232 & 0.505856 & 0.584702 \\
\hline 5 & 0.694256 & 0.48364 & 0.655249 & 0.704404 & 0.808144 \\
\hline 6 & 0.718104 & 0.674156 & 0.684001 & 0.84031 & 1.007899 \\
\hline 7 & 0.8081 & 0.976339 & 0.985538 & 0.886392 & 1.251168 \\
\hline 8 & 0.793464 & 1.022654 & 1.083476 & 1.177541 & 1.416201 \\
\hline 9 & 1.006683 & 1.140404 & 1.029003 & 1.538357 & 1.755961 \\
\hline 10 & 0.936848 & 8.555351 & 1.24804 & 1.699281 & 2.018783 \\
\hline 11 & 1.721268 & 1.285563 & 1.558277 & 1.573989 & 2.062994 \\
\hline 12 & 1.256805 & 1.948508 & 2.22967 & 1.907854 & 2.271668 \\
\hline 13 & 2.206634 & 1.948039 & 1.79316 & 2.639904 & 2.57344 \\
\hline 14 & 1.688903 & 2.326416 & 2.387203 & 2.6276 & 3.212641 \\
\hline 15 & 2.014106 & 1.893232 & 2.235541 & 2.557164 & 3.881116 \\
\hline 16 & 2.879682 & 2.875826 & 3.405001 & 3.538909 & 3.915724 \\
\hline
\end{tabular}

Table 4.44. Values of $\Delta(K, 1)$ for active ingredient PP vs. ER measure for years (2011-2015)

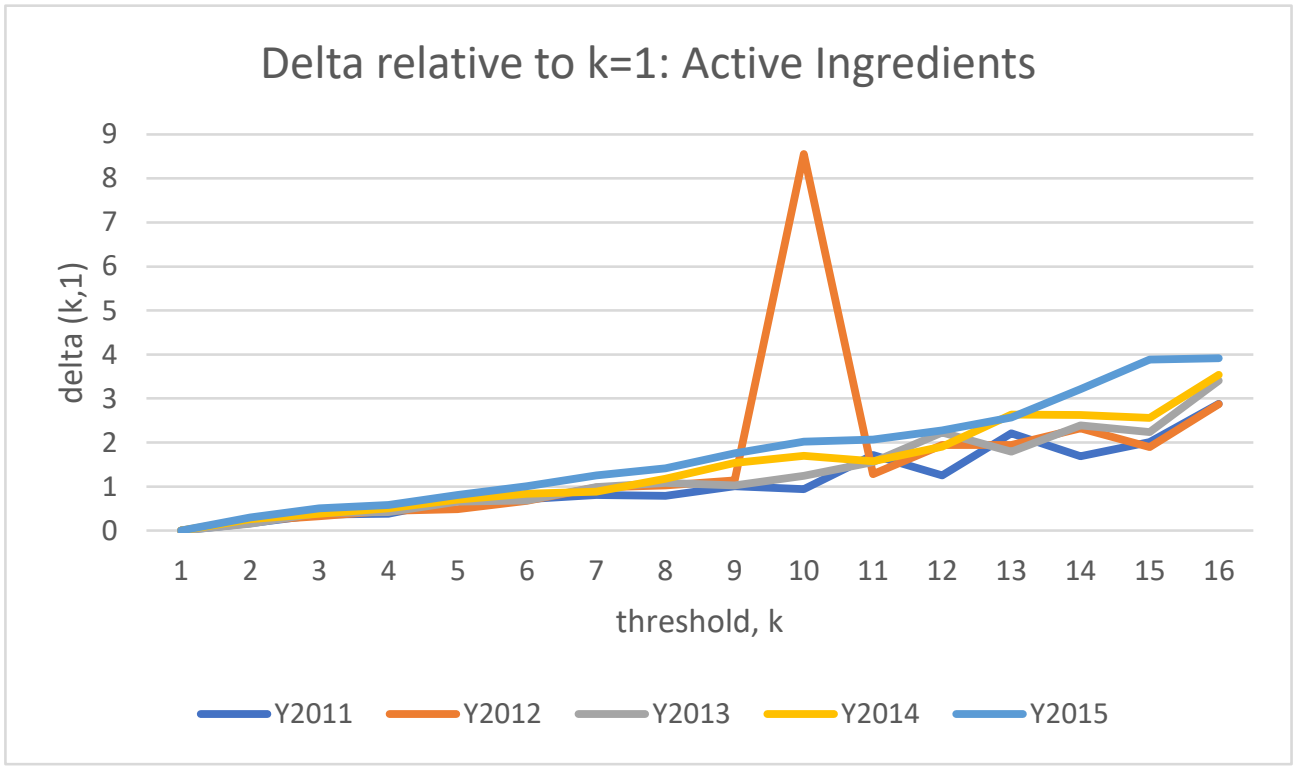

Figure 4.18. Values of $\Delta(K, 1)$ for active ingredients PP vs. ER measure of years (2011-2015)

The variation $\Delta(K, 1)$ for active ingredient polypharmacy shows similar tendency to the variation estimates for drug polypharmacy. Similar to drug polypharmacy, polypharmacy with higher number of active ingredients (i.e., greater than 1 ) is likely to be associated with more ER problems. Also, figure 4.18 shows that the variation $\Delta(K, 1)$ tend to increase over time from 2011 to 2015 . The greatest value for $\Delta(K, 1)$ is 8.5 , which was found for $k=10$ in 2012 . This is because the ER visits found for this threshold were much higher than the number of polypharmacy (\#ER=630, \#PP=296). Some of the reported ER visits were relatively high for about 8 patients (e.g., $8,7,6$, or 5 visits per year). Thus, the resulting ratio (\#ER/\#PP) for this threshold is very high 
compared to the others in 2012 and in all years. Table 4.45 and Figure 4.19 show the values of delta function for the polypharmacy of TC1 drug classes.

\begin{tabular}{|c|c|c|c|c|c|c|c|}
\hline \multirow[t]{2}{*}{$\mathrm{k}$ value } & \multicolumn{2}{|c|}{ Y2011 } & Y2012 & Y2013 & Y2014 & Y2015 & \multirow{4}{*}{ Table 4.45. Values of $\Delta(K, 1)$} \\
\hline & 1 & 0 & 0 & 0 & 0 & 0 & \\
\hline & 2 & 0.185875 & 0.207333 & 0.187966 & 0.23479 & 0.18153 & \\
\hline & 3 & 0.410034 & 0.419495 & 0.410904 & 0.387031 & 0.417995 & \\
\hline & 4 & 0.473965 & 0.57439 & 0.702658 & 0.683705 & 0.706127 & for TC1 class PP vs. ER \\
\hline & 5 & 0.67914 & 0.945249 & 0.983808 & 1.09742 & 1.144057 & measure of years \\
\hline & 6 & 1.161659 & 1.14829 & 1.231444 & 1.293912 & 1.729688 & 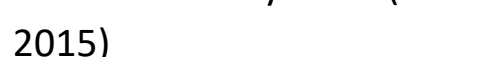 \\
\hline & 7 & 1.519644 & 1.489975 & 1.708219 & 1.860121 & 2.091849 & \\
\hline & 8 & 2.365361 & 2.184118 & 1.996787 & 1.88839 & 2.822632 & \\
\hline & 9 & 2.713373 & 2.606817 & 2.536552 & 3.502504 & 2.691316 & \\
\hline & 10 & 1.500583 & 4.205949 & 4.696988 & 4.38011 & 3.003476 & \\
\hline & 11 & 0.804932 & 2.354696 & 2.113945 & 6.765674 & 5.764784 & \\
\hline & 12 & 5.317263 & 1.477314 & 12.70136 & 2.663054 & 2.927939 & \\
\hline & 13 & -1 & \#DIV/0! & 6.473468 & -1 & -1 & \\
\hline & 14 & \#DIV/0! & \#DIV/0! & \#DIV/0! & \#DIV/0! & \#DIV/0! & \\
\hline & 15 & \#DIV/0! & \#DIV/0! & \#DIV/0! & \#DIV/0! & \#DIV/0! & \\
\hline & 16 & \#DIV/0! & \#DIV/0! & \#DIV/0! & \#DIV/0! & \#DIV/0! & \\
\hline
\end{tabular}

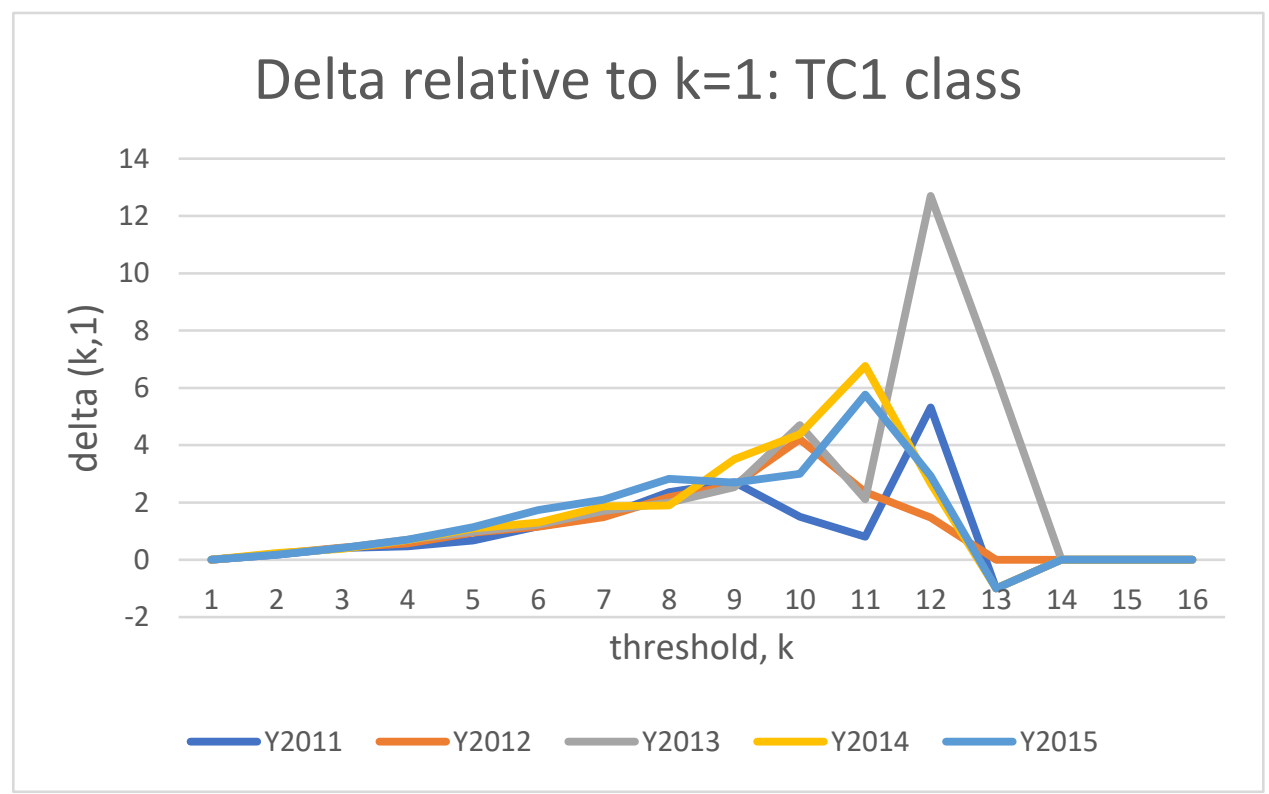

Figure 4.19. Values of $\Delta(\mathrm{K}, 1)$ for TC1 class PP vs. ER measure of years (2011-2015) 
From Table 4.45 and Figure 4.19 we can observe that increasing number of TC1 classes has positive relationship with number of emergency room admissions at the threshold range (2-10). However, for thresholds higher than 10 and less than 14 the results were fluctuated. This is explained given that there are much less polypharmacy groups for these thresholds (i.e., as thresholds increase, the number of polypharmacy sets found for these thresholds decrease). As a result to this fact, no polypharmacy sets were found for thresholds greater than 14 . The noticeable increase located for 2013 in Figure 4.19 is because there was one patient who has 6 visits and three polypharmacy sets. This contributes to increase the \#ER total to be 22 vs. 6 for \#PP polypharmacy sets and increase the final variation for $\Delta(K, 1)$ to be about 12.70. Table 4.46 and Figure 4.20 show the values of delta function for the polypharmacy of TC1 drug classes.

\begin{tabular}{|rrrrrrr|}
\hline k value $\begin{array}{r}\text { Y2011 } \\
\text { Y2012 }\end{array}$ & \multicolumn{1}{c}{ Y2013 } & \multicolumn{2}{c|}{ Y2014 } & \multicolumn{2}{c|}{ Y2015 } \\
\hline 1 & 0 & 0 & 0 & 0 & 0 \\
\hline 2 & 0.180859 & 0.223334 & 0.21779 & 0.25446 & 0.273589 \\
\hline 3 & 0.35387 & 0.354538 & 0.325613 & 0.293121 & 0.362164 \\
\hline 4 & 0.505949 & 0.412752 & 0.424582 & 0.453412 & 0.618185 \\
\hline 5 & 0.569774 & 0.645759 & 0.71072 & 0.672864 & 0.691822 \\
\hline 6 & 0.564856 & 0.842779 & 0.838142 & 0.902014 & 1.147886 \\
\hline 7 & 0.81046 & 0.232098 & 1.121623 & 1.107839 & 1.317363 \\
\hline 8 & 1.053506 & 0.946379 & 1.139427 & 1.75915 & 1.631853 \\
\hline 9 & 1.40176 & 1.180292 & 1.407035 & 1.778943 & 2.008079 \\
\hline 10 & 1.722948 & 1.581156 & 2.347312 & 1.370593 & 2.07888 \\
\hline 11 & 1.410364 & 2.331826 & 1.824076 & 2.288293 & 2.337432 \\
\hline 12 & 2.312381 & 2.346753 & 2.541634 & 2.629673 & 3.323839 \\
\hline 13 & 2.463039 & 2.896342 & 2.872186 & 2.554303 & 3.472542 \\
\hline 14 & 2.376703 & 3.839667 & 3.477216 & 4.016547 & 4.281758 \\
\hline 15 & 2.809931 & 3.052196 & 3.592593 & 3.957821 & 2.465002 \\
\hline 16 & 2.280774 & 3.826716 & 3.709416 & 4.236249 & 4.030709 \\
\hline
\end{tabular}

Table 4.46. Values of $\Delta(K, 1)$ for TC1S1 class PP vs. ER measure of years (2011-2015)

$\begin{array}{lllllll}16 & 2.280774 & 3.826716 & 3.709416 & 4.236249 & 4.030709\end{array}$

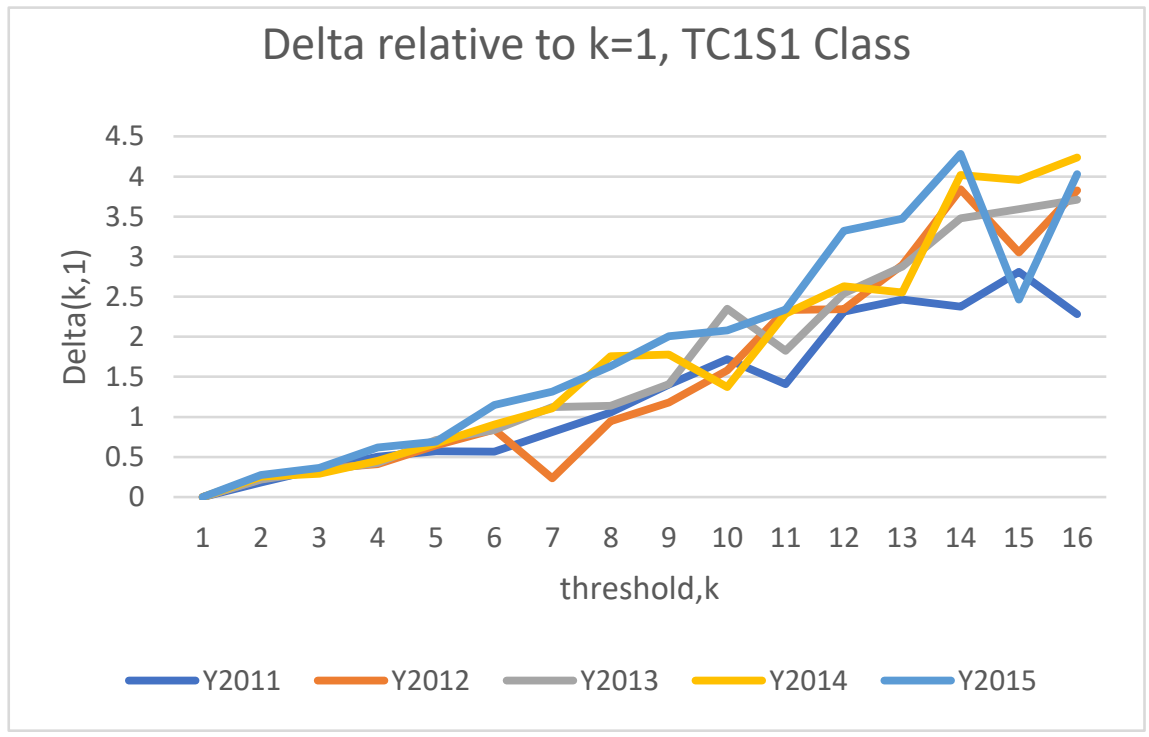

Figure 4.20. Values of $\Delta(\mathrm{K}, 1)$ for TC1S1 class PP vs. ER measure for years (2011-2015). 
The variation $\Delta(\mathrm{K}, 1)$ for TC1S1 polypharmacy generally have similar trend to that for active ingredient. In particular, \#ER visits increase with increasing number of thresholds so polypharmacy with higher number of drug classes of this type (i.e., greater than 1) is likely to be associated with more ER problems. Also, the overall increase in the variation $\Delta(K, 1)$ over time from 2011 to 2015 persists for this polypharmacy definition as shown in Figure 4.20.

We then calculate the average value for every year to get an overall view of the values over time. Table 4.47 contains the numerical values while Figure 4.21. show the graph of these values.

\begin{tabular}{|c|c|c|c|c|c|c|}
\hline $\mathrm{k}$ value & \multicolumn{2}{|c|}{ Al-Avg } & Drug-Avg & TC1-Avg & TCS1-Avg & \multirow{17}{*}{$\begin{array}{l}\text { Table 4.47. Average values of } \Delta(K, 1) \\
\text { for all PP vs. ER measure of years } \\
(2011-2015) \text {. }\end{array}$} \\
\hline & 1 & 0 & 0 & 0 & 0 & \\
\hline & 2 & 0.2185 & 0.24187 & 0.200032 & 0.223244 & \\
\hline & 3 & 0.39382 & 0.350566 & 0.411483 & 0.330026 & \\
\hline & 4 & 0.469048 & 0.51712 & 0.639817 & 0.474692 & \\
\hline & 5 & 0.669855 & 0.621935 & 0.989507 & 0.654086 & \\
\hline & 6 & 0.78378 & 0.808919 & 1.330563 & 0.860198 & \\
\hline & 7 & 0.987717 & 0.978749 & 1.76991 & 0.856638 & \\
\hline & 8 & 1.11348 & 1.189678 & 2.288172 & 1.336593 & \\
\hline & 9 & 1.300614 & 1.314252 & 2.894918 & 1.567383 & \\
\hline & 10 & 2.190311 & 1.62676 & 3.88247 & 1.818723 & \\
\hline & 11 & 1.644473 & 1.612296 & 4.092853 & 2.055386 & \\
\hline & 12 & 1.946854 & 2.589814 & 5.441164 & 2.668355 & \\
\hline & 13 & 2.242127 & 2.219799 & 0.96377 & 2.863218 & \\
\hline & 14 & 2.495532 & 2.67264 & \#DIV/0! & 3.742511 & \\
\hline & 15 & 2.503638 & 2.646897 & \#DIV/0! & 3.240996 & \\
\hline & 16 & 3.387051 & 3.769143 & \#DIV/0! & 3.810177 & \\
\hline
\end{tabular}

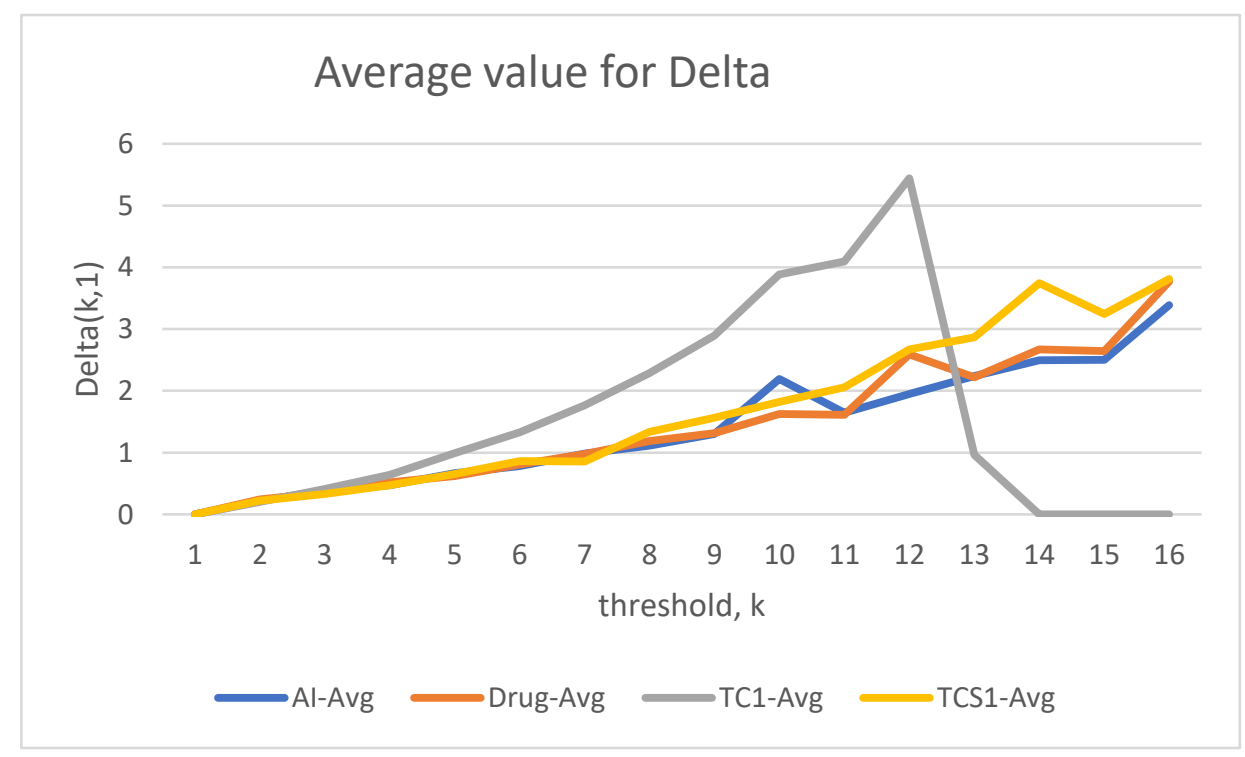

Figure 4.21. Values of $\Delta(K, 1)$ for all PP vs. ER measure of years (2011-2015) 
We can observe from Table 4.42 and Figure 4.21 that defining polypharmacy using the number of TC1 class shows the highest the average followed by the average for the definition that uses TC1S1 Followed by the average for the definition that uses drugs, and finally the average for the definition that uses active ingredients. This implies that the definition with higher level of granularity provides more specific results than the definition with lower level of granularity. As a result, defining the polypharmacy using active ingredients as the basic type should provide superior and more related associations, followed by polypharmacy of drugs followed by polypharmacy of TC1S1 drug classes, and finally polypharmacy of TC1 drug classes.

\subsubsection{Choosing Polypharmacy Thresholds}

Section 4.5.1 provides the values of our proposed function $\Delta$ with respect to the threshold 1 assuming that this threshold (which represents single drug/single active ingredient/single drug class) should be associated to the least risk in comparison to any threshold greater than 1 . Table 4.48 summarizes the average differences, showing where they crossed above $50 \%$ increase. We use these values to derive the polypharmacy thresholds based on the above assumption.

Table 4.48 Summary statistics on the values for the delta function $(\Delta(K, 1))$, and the minimum $\mathrm{k}$ value at which $\Delta(K, 1) \geq 0.50$.

\begin{tabular}{|l|l|r|r|r|r|r|c|}
\hline & k value & \multicolumn{1}{|c|}{ Y2011 } & \multicolumn{1}{|c|}{ Y2012 } & \multicolumn{1}{|c|}{ Y2013 } & \multicolumn{1}{|c|}{ Y2014 } & \multicolumn{1}{|c|}{ Y2015 } & AvgY11-Y15 \\
\hline Al & 5 & 0.694 & 0.484 & 0.655 & 0.704 & 0.808 & 0.669 \\
\hline & Avg & 1.176 & 1.742 & 1.351 & 1.523 & 1.838 & 1.526 \\
\hline & std & 0.772 & 2.045 & 0.908 & 0.978 & 1.171 & 0.983 \\
\hline TC1S1 & 5 & 0.570 & 0.646 & 0.711 & 0.673 & 0.692 & 0.658 \\
\hline & avg & 1.388 & 1.648 & 1.770 & 1.885 & 2.003 & 1.739 \\
\hline & stdv & 0.887 & 1.299 & 1.236 & 1.366 & 1.315 & 1.190 \\
\hline Drug & 4 & 0.498 & 0.461 & 0.485 & 0.525 & 0.610 & 0.516 \\
\hline & Avg & 1.217 & 1.497 & 1.465 & 1.587 & 1.820 & 1.517 \\
\hline & stdev & 0.765 & 1.091 & 1.016 & 1.126 & 1.184 & 1.005 \\
\hline TC1 & 4 & 0.474 & 0.574 & 0.703 & 0.684 & 0.706 & 0.628 \\
\hline & Avg & 1.557 & 1.601 & 2.661 & 2.251 & 2.135 & 2.041 \\
\hline & Stddev & 1.483 & 1.173 & 3.557 & 1.979 & 1.590 & 1.547 \\
\hline
\end{tabular}

As a result, we can infer that the thresholds values are 4 drugs, 5 active ingredients, 4 TC1 classes, and 5 TC1S1 classes. Thus, we can substitute the thresholds we defined in chapter 3 for our composite approach to define polypharmacy as the following:

$k_{\Delta}=4$ for drugs, $k_{\Delta} I=5$ for active ingredients, $k_{\Delta} c=4$ if we use TC1 drug class definition or $k_{\Delta} c$ $=5$ if we use TC1S1 class definition. 


\subsubsection{Redefining polypharmacy}

Now, we apply the joint consideration of the three criteria, this include:

- Strict polypharmacy definition (Using AND Logic on the three definitions)

By which we obtain the following definitions:

$\circ$ drug and $\mathrm{Al}$ and TC1 (here, $\mathrm{Al}=$ Active Ingredients)

$\circ$ drug and Al and TC1S1

- Less strict polypharmacy definition (Using OR Logic on the three definitions), this includes:

- Meeting 1 criteria of the three.
O drug OR Al
O drug OR TC1
O drug OR TC1S1
- Al OR TC1
- Al OR TC1S1
O drug OR AI OR TC1
- drug OR AI OR TC1S1

- Meeting 2 criteria of the three (with at most one of the drug class thresholds), this includes:
$O$ drug and $\mathrm{Al}$
o drug and TC1
$\circ$ drug and TC1S1
O Al and TC1
- Al and TC1S1

Table 4.49 provides the values of ER visits, the number of polypharmacy sets found in the specified thresholds, and the ratio \#ER/\#PP for each definition. Figure $\mathbf{4 . 2 2}$ shows a comparative bar graph for these definitions.

Table 4.49 Measuring \#ER/\#PP for all possible definitions of polypharmacy

\begin{tabular}{|l|r|r|r|}
\hline Definition & ER sum & PP sum & \#ER/\#PP \\
\hline drug & 31271 & 66566 & 0.470 \\
\hline Al & 27876 & 56082 & 0.497 \\
\hline TC1 & 20867 & 39920 & 0.523 \\
\hline TCS1 & 21772 & 41267 & 0.528 \\
\hline drug and Al and TC1 & 128789 & 234428 & 0.549 \\
\hline drug and Al and TC1S1 & 139857 & 260308 & 0.537 \\
\hline drug and Al & 64802 & 128048 & 0.506 \\
\hline drug and TC1 & 51409 & 96796 & 0.531 \\
\hline drug and TC1S1 & 54396 & 102525 & 0.531 \\
\hline Al and TC1 & 51828 & 96464 & 0.537 \\
\hline
\end{tabular}




\begin{tabular}{|l|r|r|r|}
\hline Al and TC1S1 & 52313 & 97661 & 0.536 \\
\hline drug OR Al OR TC1 & 80014 & 162568 & 0.492 \\
\hline drug OR AI OR TC1S1 & 80919 & 163915 & 0.494 \\
\hline drug OR Al & 59147 & 122648 & 0.482 \\
\hline drug OR TC1 & 52138 & 106486 & 0.490 \\
\hline drug OR TC1S1 & 53043 & 107833 & 0.492 \\
\hline Al OR TC1 & 48743 & 96002 & 0.508 \\
\hline Al OR TC1S1 & 49648 & 97349 & 0.510 \\
\hline
\end{tabular}

Figure 4.22. compares \#ER/\#PP for all possible definitions of polypharmacy

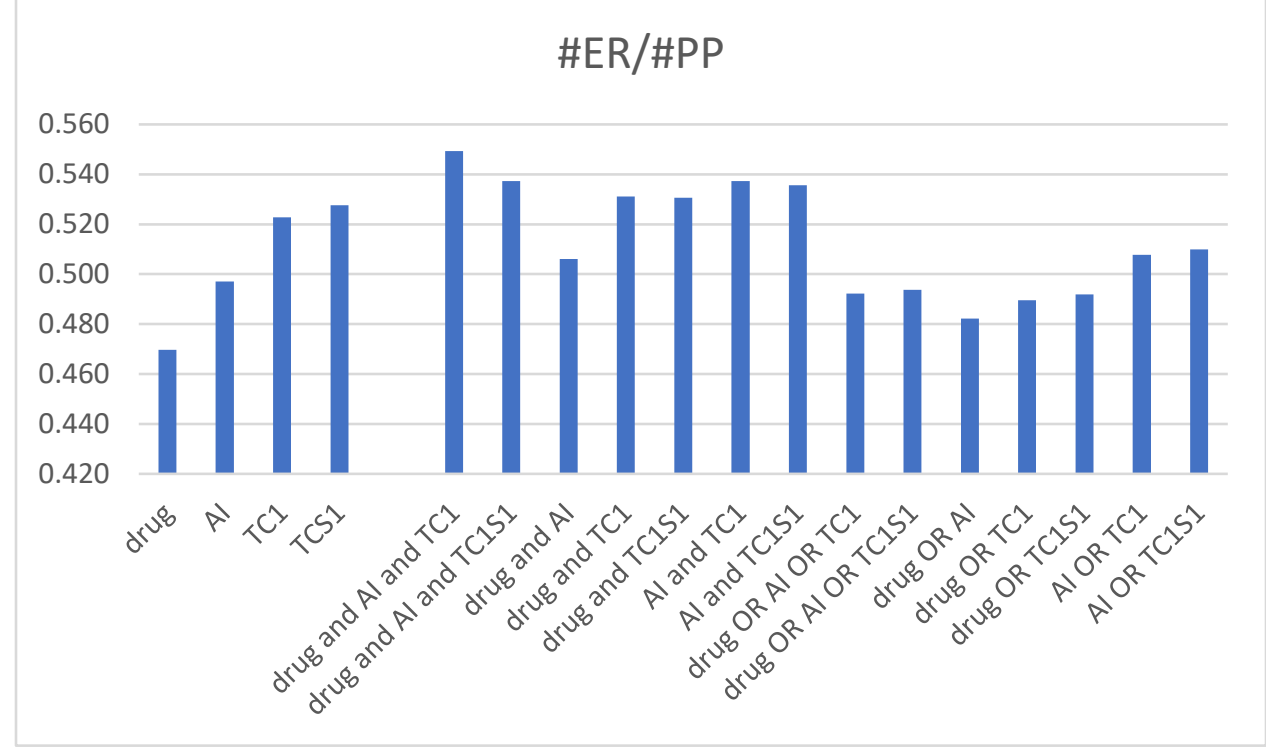

As illustrated by figure 4.22, The composite definitions have the highest values of \#ER/\#PP. This means that with respect to safety, patients that meet the three criteria jointly, (i.e, satisfy the thresholds for the definitions: drug and AI and TC1, OR the definition: drug and Al and TC1S1) may expose to significantly higher risk, when compared with patients who meet the individual definitions of polypharmacy or the relatively relaxed ones (joint by logical OR).

On the other hand, this should not eliminate the importance of the definitions formed by the logical OR since these definitions may provide additional details that could be missed when considering one individual definition. In particular, health care professional may need to highlight patients profile of polypharmacy who (not strictly) happened to meet more than a criterion rather than looking at it from one angle.

For instance, consider a patient is taking two drugs (Bacitracin, and EPIDUO FORTE). This person does not have a polypharmacy by considering the classic drug definitions and setting its threshold $k_{\Delta}$ to be three or more (i.e., $k_{\Delta}=3$ ). Likewise, these drugs belong to one drug class (DERMATOLOGICAL AGENTS) as classified by TC1S1 therapeutic classes and so this person will not have a polypharmacy if we set the threshold for this definition $k_{\Delta c}$ to be say (four or more) (i.e., $k_{\Delta c}=4$ ). Looking at this polypharmacy from either a drug point or a TC1S1 classification point 
of view will not flag any risk. However, considering the active ingredient approach, these two drugs contains 5 ingredients namely (ADAPALENE, BENZOYL PEROXIDE, BACITRACIN ZINC, NEOMYCIN SULFATE, and POLYMYXIN B SULFATE). As a result, if we use the threshold 5 for this definition (i. e., $k_{\Delta I}=5$ ), this person will have a polypharmacy using the active ingredient definition. Based on the above example, the following definitions will not capture the polypharmacy and so may not be effective enough in preventing any possibility of risk:

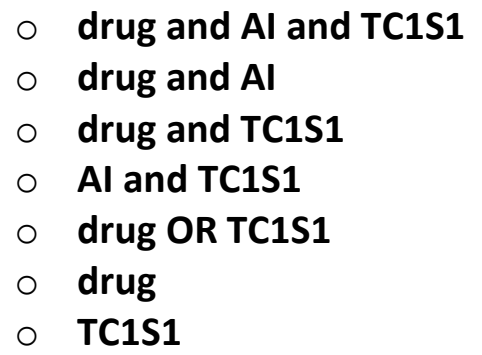

On the other hand, these definitions will capture this polypharmacy:

O drug OR AI OR TC1S1

O drug OR Al

- AI OR TC1S1

○ Al

Another example is a patient taking the four drugs (ZOVIRAX, LASTACAFT, XANAX, and SAPHRIS). These drugs contains the four active ingredients (ACYCLOVIR, ALCAFTADINE, ALPRAZOLAM, ASENAPINE MALEATE) and belong to the classes (DERMATOLOGICAL AGENTS, OPHTHALMIC PREPARATIONS, ANXIOLYTICSSEDATIVESAND HYPNOTICS, and ANTIPSYCHOTICS) as classified by TC1S1 classification scheme. Now, if we keep our thresholds choice as the example above (i.e., $k_{\Delta}=3, k_{\Delta c}=4$, and $k_{\Delta I}=5$ for drugs, TC1S1 drug class, active ingredients respectively), this patient will have polypharmacy based on drug definition and TC1S1 definition but not by the active ingredient definition.

Thus, the definitions that will capture the polypharmacy this time are:

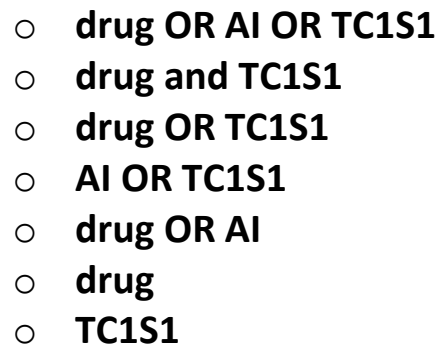

and the ones that will not do so are:

$O \quad$ drug and Al and TC1S1

$O$ drug and Al

○ Al and TC1S1

○ Al 
Therefore, and based on the above examples, we can infer that allowing the logical OR that join the polypharmacy of (drug and AI) or (TC1S1 and AI) would facilitate better observing for polypharmacy issues involved. In fact, enabling the OR logic on the three definitions would provide the safest consideration of risk even though it could be too loose to capture many polypharmacy sets as risky when they could not necessarily be in the reality. Yet, in general, it would be better not to miss any potential risk (using the OR logic) than waiting to reach high risk by meeting the three criteria (using the AND logic).

We here emphasize that the choice among these different approaches to polypharmacy could depend on the specific application and the risk that the health care specialists think is appropriate for patients.

\section{Chapter 5: Conclusion}

In this study, we have used three methods to define polypharmacy including polypharmacy of drugs and polypharmacy of drug-classes, which have been considered in prior work, as well as our proposed definition by the drug active ingredients. Independent of these definitions, our results indicated a general increase in polypharmacy problems reflected by ER visits with increasing number of drugs/drug classes/or active ingredients. However, the threshold found for those polypharmacy varied with the specific basic elements used in the definition. This variation resulted in our idea of defining polypharmacy in a composite manner by combining three criteria that determine the thresholds for each definition. Our results also included quantitative analysis of the occurrence of single-elements (e.g., single drugs, single drug classes, or single active ingredients), and the co-occurrence of multiple elements of the same basic type (e.g., two-drugs, two drug classes, two active ingredients, three-drugs, three drug classes, three active ingredients, etc.). This provides a basis for future studies to further analyze the incidences of common drugs, active ingredients, drug class polypharmacy combinations, as well as their richness to advance the study of their implications on patient's safety. In conclusion, this work forms a framework for healthcare specialists to observe the impact of each polypharmacy definition and their respective associations with emergency room (ER) visits, and which combinations of drugs, of drug classes, or of active ingredients are likely to result in significant hospitalization, and thus should be avoided.

The following are Key points summarizes the contributions from this work:

- If we must use one element to define polypharmacy, then active ingredients should be used.

- If we must use a drug class to define polypharmacy, then TC1S1 should be used.

- Whichever element type is used, we have proposed an automated way to determine the thresholds. 
- For strict polypharmacy definition, we should use the AND logic on the different elements using the proposed thresholds.

- For less strict definition, we use the OR logic.

- Analysis of the richness or overreprestation of the polypharmacy elements showed that the richness of ER occurrence with polypharmacy when considered with the direct polypharmacy occurrence richness provides a more insight on the association between polypharmacy and health problems.

\subsection{Limitations}

- Our assumption that patients actually take all medications they were prescribed pose a limitation as this may not hold at all times.

- The method used to extract active ingredients depends on exact matching of NDCs and drug names. Drug names are not necessarily consistent within the two dataset (i.e., MEPS dataset and NHANES dataset) used for this study. As a result, many active ingredients were not found and were substituted by drug NDC in the polypharmacy of ingredients. This would affect the results of polypharmacy of active ingredients with respect to the number of elements and the actual polypharmacy components.

- ER visits extracted from MEPS files represents the total ER visits for a patient within the MEPS year. In particular, there is no indication that those ER visits happened prior or later than the polypharmacy sets found in the rounds. Thus, weather the polypharmacy caused or ( is caused by) the ER visits is unknown.

\subsection{Future Work}

The possible directions for future work can be listed as follows:

- Practitioners can study risk stratification of patients based on our methodology of determining polypharmacy thresholds. For example, further experiments can be done on thresholds of the three definitions of polypharmacy and their associations with specific health outcomes, such as specific chronic diseases.

- This work will benefit researchers who are interested in studying polypharmacy safety to prioritize the most common combinations of elements (two-drugs, two drug classes, two active ingredients, three-drugs, three drug classes, three active ingredients, etc) in their subsequent studies and consequently drive observations that are more connected to the majority of community problems. Another direction with the provided repository is mapping drugs to possible diseases and summarize the comorbidities that reflect the trends in the prescription claims data.

- Examine the list of elements involved in each polypharmacy definition against data sets of adverse drug events and drug interactions to determine whether they are potential to cause inappropriate therapy problems and create models by which the suspicious patterns of medications can be learned using data mining techniques.

- Involve patients' characteristics such as ethnicity, sex, and age to observe associations with regard to demographics. 


\section{References}

[1] Goldberg, J. F., Kurita, K., Hoblyn, J. C., Ghaemi, S. N., Perlis, R. H., Miklowitz, D. J., ... \& Thase, M. E. (2009). Depressive illness burden associated with complex polypharmacy in patients with bipolar disorder: findings from the STEP-BD. The Journal of clinical psychiatry, 70(2), 155-162.

[2] Maher, R. L., Hanlon, J., \& Hajjar, E. R. (2014). Clinical consequences of polypharmacy in elderly. Expert opinion on drug safety, 13(1), 57-65.

[3] Quinn, K. J., \& Shah, N. H. (2017). A dataset quantifying polypharmacy in the United States. Scientific data, 4, 170167.

[4] Dai, D., Feinstein, J. A., Morrison, W., Zuppa, A. F., \& Feudtner, C. (2016). Epidemiology of Polypharmacy and Potential Drug-Drug Interactions among Pediatric Patients in Intensive Care Units of US Children's Hospitals. Pediatric critical care medicine: a journal of the Society of Critical Care Medicine and the World Federation of Pediatric Intensive and Critical Care Societies, 17(5), e218.

[5] Dagli, R. J., \& Sharma, A. (2014). Polypharmacy: a global risk factor for elderly people. Journal of international oral health: $J \mathrm{OH}, 6(6)$, i.

[6] Rambhade, S., Chakarborty, A., Shrivastava, A., Patil, U. K., \& Rambhade, A. (2012). A survey on polypharmacy and use of inappropriate medications. Toxicology international, 19(1), 68.

[7] Bourgeois, F. T., Shannon, M. W., Valim, C., \& Mandl, K. D. (2010). Adverse drug events in the outpatient setting: an 11-year national analysis. Pharmacoepidemiology and drug safety, 19(9), 901-910.

[8] Neel, A. B., \& Hogan, B. (2012). Are Your Prescriptions Killing You?: How to Prevent Dangerous Interactions, Avoid Deadly Side Effects, and be Healthier with Fewer Drugs. Simon and Schuster.

[9] da Silva, B. A., \& Krishnamurthy, M. (2016). The alarming reality of medication error: a patient case and review of Pennsylvania and National data. Journal of community hospital internal medicine perspectives, 6(4), 31758.

[10] Hedegaard H, Warner M, Miniño AM. Drug overdose deaths in the United States, 19992016. NCHS Data Brief, no 294. Hyattsville, MD: National Center for Health Statistics. 2017.

[11] Lebanon, N. H., \& Hanover, N. H. (2000). How many deaths are due to medical error? Getting the number right. Eff Clin Pract, 6, 277-283.

[12] Viktil, K. K., Blix, H. S., \& Reikvam, Å. (2008). The Janus face of polypharmacy-overuse versus underuse of medication. Norsk Epidemiologi, 18(2).

[13] Qato, D. M., Wilder, J., Schumm, L. P., Gillet, V., \& Alexander, G. C. (2016). Changes in prescription and over-the-counter medication and dietary supplement use among older adults in the United States, 2005 vs 2011. JAMA internal medicine, 176(4), 473-482.

[14] Kantor, E. D., Rehm, C. D., Haas, J. S., Chan, A. T., \& Giovannucci, E. L. (2015). Trends in prescription drug use among adults in the United States from 1999-2012. Jama, 314(17), 1818-1830. 
[15] Jyrkkä, J., Enlund, H., Korhonen, M. J., Sulkava, R., \& Hartikainen, S. (2009). Polypharmacy status as an indicator of mortality in an elderly population. Drugs \& aging, 26(12), 10391048.

[16] Steinman, M. A. (2016). Polypharmacy-time to get beyond numbers. JAMA internal medicine, 176(4), 482-483.

[17] Vyas, A., Pan, X., \& Sambamoorthi, U. (2012). Chronic condition clusters and polypharmacy among adults. International journal of family medicine, 2012.

[18] Salwe, K. J., Kalyansundaram, D., \& Bahurupi, Y. (2016). A study on polypharmacy and potential drug-drug interactions among elderly patients admitted in department of medicine of a tertiary care hospital in Puducherry. Journal of clinical and diagnostic research: JCDR, 10(2), FC06.

[19] Meraya, A. M., Dwibedi, N., \& Sambamoorthi, U. (2016). Peer Reviewed: Polypharmacy and Health-Related Quality of Life Among US Adults With Arthritis, Medical Expenditure Panel Survey, 2010-2012. Preventing chronic disease, 13.

[20] Richardson, K., Bennett, K., \& Kenny, R. A. (2014). Polypharmacy including falls riskincreasing medications and subsequent falls in community-dwelling middle-aged and older adults. Age and ageing, 44(1), 90-96.

[21] Barnett, K., McCowan, C., Evans, J. M. M., Gillespie, N. D., Davey, P. G., \& Fahey, T. (2011). Prevalence and outcomes of use of potentially inappropriate medicines in older people: cohort study stratified by residence in nursing home or in the community. BMJ quality \& safety, 20(3), 275-281.

[22] World Health Organization. (2006). WHO Collaborating Centre for Drug Statistics Methodology: ATC classification index with DDDs and Guidelines for ATC classification and DDD assignment. Oslo, Norway: Norwegian Institute of Public Health.

[23] Sutherland, J. J., Daly, T. M., Liu, X., Goldstein, K., Johnston, J. A., \& Ryan, T. P. (2015). Coprescription trends in a large cohort of subjects predict substantial drug-drug interactions. PloS one, 10(3), e0118991.

[24] Abe, T., Tamiya, N., Kitahara, T., Hasegawa, Y., \& Tokuda, Y. (2014). Polypharmacy as a risk factor for hospital admission among elderly using emergency transport. BMC health services research, 14(2), P2.

[25] Zarowitz, B. J., Stebelsky, L. A., Muma, B. K., Romain, T. M., \& Peterson, E. L. (2005). Reduction of high-risk polypharmacy drug combinations in patients in a managed care setting. Pharmacotherapy: The Journal of Human Pharmacology and Drug Therapy, 25(11), 1636-1645.

[26] Garfinkel, D., Ilhan, B., \& Bahat, G. (2015). Routine deprescribing of chronic medications to combat polypharmacy. Therapeutic advances in drug safety, 6(6), 212-233.

[27] Grando, A., Farrish, S., Boyd, C., \& Boxwala, A. (2012). Ontological approach for safe and effective polypharmacy prescription. In AMIA Annual Symposium Proceedings (Vol. 2012, p. 291). American Medical Informatics Association.

[28] Makar, M., Ghassemi, M., Cutler, D. M., \& Obermeyer, Z. (2015). Short-term mortality prediction for elderly patients using Medicare claims data. International journal of machine learning and computing, 5(3), 192. 
[29] Medical Expenditure Panel Survey. (n.d.). Retrieved from https://meps.ahrq.gov/mepsweb/.

[30] Multum Lexicon Drug Database. (n.d.). Retrieved from http://www.multum.com/Lexicon.htm.

[31] Multum Lexicon Drug Database. (2007). National Health and Nutrition Examination Survey: 1988-2010 data documentation, codebook, and frequencies, prescription medications - drug information (RXQ_Drug). Retrieved from http://www.cdc.gov/nchs/nhanes/nhanes1999-2000/RXQ_DRUG.htm.

[32] Congress.gov (2016). H.R.34 - 21st Century Cures Act. Retrieved from https://www.congress.gov/bill/114th-congress/house-bill/34/text 


\section{Appendices}

\section{A TC1S1 Class Codes and Names}

\begin{tabular}{|c|c|}
\hline Class Code & Class Name \\
\hline-1 & INAPPLICABLE \\
\hline 2 & AMEBICIDES \\
\hline 3 & ANTHELMINTICS \\
\hline 4 & ANTIFUNGALS \\
\hline 5 & ANTIMALARIAL AGENTS \\
\hline 6 & ANTITUBERCULOSIS AGENTS \\
\hline 7 & ANTIVIRAL AGENTS \\
\hline 9 & CEPHALOSPORINS \\
\hline 10 & LEPROSTATICS \\
\hline 11 & MACROLIDE DERIVATIVES \\
\hline 12 & MISCELLANEOUS ANTIBIOTICS \\
\hline 13 & PENICILLINS \\
\hline 14 & QUINOLONES \\
\hline 15 & SULFONAMIDES \\
\hline 16 & TETRACYCLINES \\
\hline 17 & URINARY ANTI-INFECTIVES \\
\hline 18 & AMINOGLYCOSIDES \\
\hline 19 & ANTIHYPERLIPIDEMIC AGENTS \\
\hline 21 & ALKYLATING AGENTS \\
\hline 23 & ANTIMETABOLITES \\
\hline 24 & ANTINEOPLASTIC HORMONES \\
\hline 25 & MISCELLANEOUS ANTINEOPLASTICS \\
\hline 26 & MITOTIC INHIBITORS \\
\hline 27 & RADIOPHARMACEUTICALS \\
\hline 34 & IN VIVO DIAGNOSTIC BIOLOGICALS \\
\hline 36 & RECOMBINANT HUMAN ERYTHROPOIETINS \\
\hline 42 & ANGIOTENSIN CONVERTING ENZYME INHIBITORS \\
\hline 43 & ANTIADRENERGIC AGENTSPERIPHERALLY ACTING \\
\hline 44 & ANTIADRENERGIC AGENTSCENTRALLY ACTING \\
\hline 45 & ANTIANGINAL AGENTS \\
\hline 46 & ANTIARRHYTHMIC AGENTS \\
\hline 47 & BETA-ADRENERGIC BLOCKING AGENTS \\
\hline 48 & CALCIUM CHANNEL BLOCKING AGENTS \\
\hline 49 & DIURETICS \\
\hline 51 & MISCELLANEOUS CARDIOVASCULAR AGENTS \\
\hline 53 & VASODILATORS \\
\hline 54 & VASOPRESSORS \\
\hline 55 & ANTIHYPERTENSIVE COMBINATIONS \\
\hline
\end{tabular}




\begin{tabular}{|l|l|}
\hline 56 & ANGIOTENSIN II INHIBITORS \\
\hline 58 & ANALGESICS \\
\hline 64 & ANTICONVULSANTS \\
\hline 65 & ANTIEMETIC/ANTIVERTIGO AGENTS \\
\hline 66 & ANTIPARKINSON AGENTS \\
\hline 67 & ANXIOLYTICSSEDATIVESAND HYPNOTICS \\
\hline 71 & CNS STIMULANTS \\
\hline 73 & MUSCLE RELAXANTS \\
\hline 80 & MISCELLANEOUS CENTRAL NERVOUS SYSTEM \\
\hline 82 & ANTICOAGULANTS \\
\hline 83 & ANTIPLATELET AGENTS \\
\hline 85 & MISCELLANEOUS COAGULATION MODIFIERS \\
\hline 88 & ANTACIDS \\
\hline 90 & ANTIDIARRHEALS \\
\hline 91 & DIGESTIVE ENZYMES \\
\hline 92 & GALLSTONE SOLUBILIZING AGENTS \\
\hline 94 & H2 ANTAGONISTS \\
\hline 95 & LAXATIVES \\
\hline 96 & MISCELLANEOUS GI AGENTS \\
\hline 98 & ADRENAL CORTICAL STEROIDS \\
\hline 99 & ANTIDIABETIC AGENTS \\
\hline 101 & SEX HORMONES \\
\hline 103 & THYROID HORMONES \\
\hline 104 & IMMUNOSUPPRESSIVE AGENTS \\
\hline 106 & ANTIDOTES \\
\hline 107 & CHELATING AGENTS \\
\hline 110 & MISCELLANEOUS UNCATEGORIZED AGENTS \\
\hline 112 & RADIOCONTRAST AGENTS \\
\hline 116 & IRON PRODUCTS \\
\hline 117 & MINERALS AND ELECTROLYTES \\
\hline 119 & VITAMINS \\
\hline 120 & VITAMIN AND MINERAL COMBINATIONS \\
\hline 121 & INTRAVENOUS NUTRITIONAL PRODUCTS \\
\hline 123 & ANTIHISTAMINES \\
\hline 124 & ANTITUSSIVES \\
\hline 125 & BRONCHODILATORS \\
\hline 127 & DECONGESTANTS \\
\hline 128 & EXPECTORANTS \\
\hline 129 & MISCELLANEOUS RESPIRATORY AGENTS \\
\hline 131 & RESPIRATORY INHALANT PRODUCTS \\
\hline & ANTIASTHMATIC COMBINATIONS \\
\hline UPPER RESPIRATORY COMBINATIONS \\
\hline
\end{tabular}




\begin{tabular}{|l|l|}
\hline 134 & ANORECTAL PREPARATIONS \\
\hline 135 & ANTISEPTIC AND GERMICIDES \\
\hline 136 & DERMATOLOGICAL AGENTS \\
\hline 146 & MOUTH AND THROAT PRODUCTS \\
\hline 147 & OPHTHALMIC PREPARATIONS \\
\hline 148 & OTIC PREPARATIONS \\
\hline 150 & STERILE IRRIGATING SOLUTIONS \\
\hline 151 & VAGINAL PREPARATIONS \\
\hline 192 & ANTIRHEUMATICS \\
\hline 194 & ANTIGOUT AGENTS \\
\hline 219 & NUTRACEUTICAL PRODUCTS \\
\hline 220 & HERBAL PRODUCTS \\
\hline 240 & LINCOMYCIN DERIVATIVES \\
\hline 243 & LEUKOTRIENE MODIFIERS \\
\hline 247 & NASAL PREPARATIONS \\
\hline 249 & ANTIDEPRESSANTS \\
\hline 251 & ANTIPSYCHOTICS \\
\hline 253 & ANOREXIANTS \\
\hline 263 & IMPOTENCE AGENTS \\
\hline 264 & URINARY ANTISPASMODICS \\
\hline 265 & URINARY PH MODIFIERS \\
\hline 266 & MISCELLANEOUS GENITOURINARY TRACT AGENTS \\
\hline 272 & PROTON PUMP INHIBITORS \\
\hline 277 & 5-AMINOSALICYLATES \\
\hline 284 & VISCOSUPPLEMENTATION AGENTS \\
\hline 288 & 5-ALPHA-REDUCTASE INHIBITORS \\
\hline 289 & ANTIHYPERURICEMIC AGENTS \\
\hline 293 & GLUCOSE ELEVATING AGENTS \\
\hline 295 & GROWTH HORMONES \\
\hline 303 & AGENTS FOR PULMONARY HYPERTENSION \\
\hline 312 & CHOLINERGIC AGONISTS \\
\hline 313 & CHOLINESTERASE INHIBITORS \\
\hline 320 & SMOKING CESSATION AGENTS \\
\hline 340 & ALDOSTERONE RECEPTOR ANTAGONISTS \\
\hline 342 & RENIN INHIBITORS \\
\hline 354 & H. PYLORI ERADICATION AGENTS \\
\hline 355 & FUNCTIONAL BOWEL DISORDER AGENTS \\
\hline 359 & PERIPHERALLY ACTING ANTIOBESITY AGENTS \\
\hline 378 & DRUGS USED IN ALCOHOL \\
\hline & MULTIKINASE INHIBITORS \\
\hline BCR-ABL TYROSINE KINASE INHIBITORS \\
\hline EGFR INHIBITORS \\
\hline
\end{tabular}




\begin{tabular}{|l|l|}
\hline 405 & HER2 INHIBITORS \\
\hline 409 & BONE RESORPTION INHIBITORS \\
\hline 411 & CALCITONIN \\
\hline 412 & UTEROTONIC AGENTS \\
\hline 413 & ANTIGONADOTROPIC AGENTS \\
\hline 414 & ANTIDIURETIC HORMONES \\
\hline 418 & PARATHYROID HORMONE AND ANALOGS \\
\hline 419 & GONADOTROPIN-RELEASING HORMONE ANTAGONIST \\
\hline 422 & ANTITHYROID AGENTS \\
\hline 426 & SYNTHETIC OVULATION STIMULANTS \\
\hline 437 & IMMUNOSTIMULANTS \\
\hline
\end{tabular}




\section{B Associations of polypharmacy results with ER}

Table 1: Top 30 single drugs in the dataset ranked by the corresponding ER visits

\begin{tabular}{|c|c|c|c|c|c|c|c|c|}
\hline & \multirow[b]{2}{*}{ Single drug } & \multicolumn{5}{|c|}{ Number of ER } & \multirow{2}{*}{$\begin{array}{l}\text { ER } \\
\text { Total } \\
\end{array}$} & \multirow[b]{2}{*}{ \#ER/\#Occ } \\
\hline & & 2011 & 2012 & 2013 & 2014 & 2015 & & \\
\hline 1 & AMOXICILLIN & 199 & 251 & 253 & 261 & 226 & 1,190 & 0.266 \\
\hline 2 & AZITHROMYCIN & 210 & 243 & 216 & 183 & 39 & 891 & 0.280 \\
\hline 3 & HYDROCO/APAP & 148 & 145 & 126 & 162 & 117 & 698 & 0.564 \\
\hline 4 & IBUPROFEN & 112 & 140 & 138 & 149 & 152 & 691 & 0.413 \\
\hline 5 & APAP/CODEINE & 67 & 59 & 83 & 50 & 45 & 304 & 0.495 \\
\hline 6 & LISINOPRIL & 66 & 46 & 69 & 70 & 40 & 291 & 0.168 \\
\hline 7 & ALBUTEROL & 34 & 73 & 68 & 51 & 49 & 275 & 0.350 \\
\hline 8 & CEPHALEXIN & 40 & 28 & 50 & 51 & 27 & 196 & 0.364 \\
\hline 9 & PREDNISONE & 32 & 42 & 45 & 48 & 23 & 190 & 0.436 \\
\hline 10 & AMOX/K CLAV & & 23 & 40 & 34 & 90 & 187 & 0.386 \\
\hline 11 & LEVOTHYROXIN & 24 & 47 & 30 & 36 & 40 & 177 & 0.104 \\
\hline 12 & OXYCOD/APAP & 30 & 41 & 25 & 33 & 46 & 175 & 0.540 \\
\hline 13 & NAPROXEN & 31 & 26 & 40 & 33 & 44 & 174 & 0.353 \\
\hline 14 & ONDANSETRON & 19 & 28 & 45 & 46 & 29 & 167 & 0.658 \\
\hline 15 & OMEPRAZOLE & 19 & 36 & 37 & 34 & 28 & 154 & 0.214 \\
\hline 16 & PRENATAL & 25 & 33 & 36 & 28 & 3 & 125 & 0.463 \\
\hline 17 & CYCLOBENZAPR & 29 & 19 & 25 & 21 & 28 & 122 & 0.469 \\
\hline 18 & OXYCODONE & 15 & 28 & 20 & 33 & 19 & 115 & 0.545 \\
\hline 19 & METFORMIN & 35 & 32 & 11 & 12 & 24 & 114 & 0.146 \\
\hline 20 & PROAIR HFA & & 22 & 40 & 25 & 26 & 113 & 0.251 \\
\hline 21 & TRAMADOL HCL & & 27 & 18 & 32 & 33 & 110 & 0.444 \\
\hline 22 & SIMVASTATIN & 19 & 25 & 20 & 22 & 20 & 106 & 0.105 \\
\hline 23 & CIPROFLOXACN & 17 & 21 & 24 & 16 & 24 & 102 & 0.338 \\
\hline 24 & RANITIDINE & 16 & 36 & 14 & 19 & 14 & 99 & 0.303 \\
\hline 25 & SERTRALINE & 29 & 12 & 22 & 22 & 12 & 97 & 0.180 \\
\hline 26 & PENICILLN VK & & 17 & 24 & 36 & 17 & 94 & 0.349 \\
\hline 27 & CITALOPRAM & 24 & 17 & 25 & 15 & 9 & 90 & 0.200 \\
\hline 28 & HYDROCHLOROT & 10 & 18 & 17 & 17 & 23 & 85 & 0.184 \\
\hline 29 & PROMETHAZINE & 27 & 18 & 20 & 12 & 8 & 85 & 0.455 \\
\hline 30 & METHYLPHENID & 3 & 30 & 15 & 17 & 17 & 82 & 0.118 \\
\hline
\end{tabular}


Table 2: Top 30 two-drug polypharmacy ranked by ER visits

\begin{tabular}{|c|c|c|c|c|c|c|c|c|}
\hline & \multirow[b]{2}{*}{ Polypharmacy of two-drugs } & \multicolumn{5}{|c|}{ Number of ER } & \multirow{2}{*}{$\begin{array}{l}\text { ER } \\
\text { Total }\end{array}$} & \multirow[b]{2}{*}{ \#ER/\#Occ } \\
\hline & & 2011 & 2012 & 2013 & 2014 & 2015 & & \\
\hline 1 & HYDROCO/APAP--IBUPROFEN & 13 & 29 & 14 & 58 & 33 & 147 & 0.835 \\
\hline 2 & AMOXICILLIN--IBUPROFEN & 16 & 18 & 26 & 14 & 20 & 94 & 0.320 \\
\hline 3 & AMOXICILLIN--HYDROCO/APAP & 10 & 6 & 19 & 17 & 12 & 64 & 0.408 \\
\hline 4 & APAP/CODEINE--AZITHROMYCIN & 8 & 19 & 20 & 9 & 0 & 56 & 0.483 \\
\hline 5 & ALBUTEROL--AMOXICILLIN & 10 & 13 & 9 & 13 & 11 & 56 & 0.667 \\
\hline 6 & AMOXICILLIN--AZITHROMYCIN & 16 & 3 & 16 & 13 & 6 & 54 & 0.563 \\
\hline 7 & AZITHROMYCIN--HYDROCO/APAP & 7 & 18 & 7 & 14 & 7 & 53 & 0.639 \\
\hline 8 & CYCLOBENZAPR--HYDROCO/APAP & 11 & 5 & 4 & 18 & 14 & 52 & 0.675 \\
\hline 9 & CYCLOBENZAPR--IBUPROFEN & 5 & 15 & 10 & 8 & 12 & 50 & 0.610 \\
\hline 10 & IBUPROFEN--OXYCOD/APAP & 6 & 9 & 16 & 11 & 5 & 47 & 0.701 \\
\hline 11 & IBUPROFEN--PREDNISONE & 1 & 0 & 3 & 42 & 0 & 46 & 3.286 \\
\hline 12 & AMLODIPINE--LISINOPRIL & 4 & 5 & 1 & 16 & 17 & 43 & 0.301 \\
\hline 13 & AZITHROMYCIN-IBUPROFEN & 8 & 9 & 8 & 15 & 3 & 43 & 0.358 \\
\hline 14 & CLINDAMYCIN--HYDROCO/APAP & 3 & 9 & 9 & 11 & 11 & 43 & 1.049 \\
\hline 15 & AZITHROMYCIN--PREDNISONE & 5 & 7 & 12 & 5 & 7 & 36 & 0.571 \\
\hline 16 & APAP/CODEINE--IBUPROFEN & 9 & 0 & 6 & 10 & 11 & 36 & 0.750 \\
\hline 17 & HYDROCO/APAP--PROMETHAZINE & 7 & 6 & 3 & 11 & 3 & 30 & 1.304 \\
\hline 18 & LISINOPRIL--SIMVASTATIN & 10 & 6 & 3 & 5 & 5 & 29 & 0.131 \\
\hline 19 & IBUPROFEN--TRAMADOL HCL & & 9 & 3 & 6 & 11 & 29 & 1.115 \\
\hline 20 & ALBUTEROL--PREDNISONE & 10 & 10 & 2 & 3 & 3 & 28 & 0.875 \\
\hline 21 & CYCLOBENZAPR--NAPROXEN & 3 & 7 & 10 & 6 & 1 & 27 & 0.409 \\
\hline 22 & ALBUTEROL--AZITHROMYCIN & 12 & 4 & 3 & 3 & 4 & 26 & 0.441 \\
\hline 23 & HYDROCO/APAP--PENICILLN VK & & 3 & 10 & 7 & 6 & 26 & 0.491 \\
\hline 24 & AMOXICILLIN--PREDNISONE & 4 & 1 & 5 & 15 & 1 & 26 & 0.542 \\
\hline 25 & AMLODIPINE--HYDROCHLOROT & 1 & 6 & 3 & 8 & 5 & 23 & 0.240 \\
\hline 26 & CEPHALEXIN--HYDROCO/APAP & 9 & 7 & 4 & 3 & 0 & 23 & 0.451 \\
\hline 27 & AMOXICILLIN--APAP/CODEINE & 4 & 1 & 11 & 3 & 3 & 22 & 0.349 \\
\hline 28 & SERTRALINE--TRAZODONE & 1 & 7 & 0 & 13 & 0 & 21 & 0.840 \\
\hline 29 & HYDROCO/APAP--NAPROXEN & 2 & 4 & 4 & 7 & 3 & 20 & 0.541 \\
\hline 30 & HYDROCO/APAP--OXYCOD/APAP & 0 & 5 & 7 & 7 & 1 & 20 & 0.800 \\
\hline
\end{tabular}


Table 3: Top 30 three-drug polypharmacy ranked by ER visits

\begin{tabular}{|c|c|c|c|c|c|c|c|c|}
\hline & \multirow[b]{2}{*}{ Polypharmacy of three-drugs } & \multicolumn{5}{|c|}{ Number of ER } & \multirow{2}{*}{$\begin{array}{l}\text { ER } \\
\text { Total }\end{array}$} & \multirow[b]{2}{*}{$\begin{array}{c}\text { \#ER/\# } \\
\text { Occ }\end{array}$} \\
\hline & & 2011 & 2012 & 2013 & 2014 & 2015 & & \\
\hline 1 & $\begin{array}{l}\text { CYCLOBENZAPR--HYDROCO/APAP-- } \\
\text { IBUPROFEN }\end{array}$ & 5 & 3 & 9 & 3 & 7 & 27 & 0.931 \\
\hline 2 & $\begin{array}{l}\text { ATENOLOL--CARISOPRODOL-- } \\
\text { HYDROCHLOROT }\end{array}$ & & & 24 & & & 24 & 24 \\
\hline 3 & IBUPROFEN--LANTUS--OMEPRAZOLE & & 10 & 11 & & & 21 & 10.5 \\
\hline 4 & $\begin{array}{l}\text { AMLODIPINE--METOPROLOL-- } \\
\text { VALSART/HCTZ }\end{array}$ & & & 12 & 0 & & 12 & 2.4 \\
\hline 5 & $\begin{array}{l}\text { DIVALPROEX--LEVETIRACETA-- } \\
\text { OXCARBAZEPIN }\end{array}$ & & & 12 & & & 12 & 4 \\
\hline 6 & $\begin{array}{l}\text { CLINDAMYCIN--CYCLOBENZAPR-- } \\
\text { OXYCODONE }\end{array}$ & & & 12 & & & 12 & 12 \\
\hline 7 & GLIPIZIDE--LISINOPRIL--METFORMIN & 2 & 0 & 5 & 0 & 4 & 11 & 0.333 \\
\hline 8 & $\begin{array}{l}\text { ALBUTEROL--AMOXICILLIN-- } \\
\text { PREDNISONE }\end{array}$ & 4 & & 7 & 0 & 0 & 11 & 2.2 \\
\hline 9 & $\begin{array}{l}\text { AMOXICILLIN--HYDROCO/APAP-- } \\
\text { IBUPROFEN }\end{array}$ & 3 & 0 & 6 & 1 & 0 & 10 & 0.5 \\
\hline 10 & $\begin{array}{l}\text { APAP/CODEINE--AZITHROMYCIN-- } \\
\text { IBUPROFEN }\end{array}$ & 0 & 10 & 0 & 0 & & 10 & 1.666 \\
\hline 11 & $\begin{array}{l}\text { CEPHALEXIN--HYDROCO/APAP-- } \\
\text { IBUPROFEN }\end{array}$ & & & 1 & 9 & & 10 & 2 \\
\hline 12 & $\begin{array}{l}\text { MIRTAZAPINE--PRAVASTATIN-- } \\
\text { TERAZOSIN }\end{array}$ & & 2 & 8 & & & 10 & 3.333 \\
\hline 13 & $\begin{array}{l}\text { LISINOPRIL--PERCOCET-- } \\
\text { SIMVASTATIN }\end{array}$ & & 10 & & & & 10 & 10 \\
\hline 14 & $\begin{array}{l}\text { AMOXICILLIN--APAP/CODEINE-- } \\
\text { IBUPROFEN }\end{array}$ & 0 & 3 & 4 & 2 & 0 & 9 & 1 \\
\hline 15 & $\begin{array}{l}\text { FEXOFENADINE--LEVETIRACETA-- } \\
\text { VITAMIN D }\end{array}$ & & 9 & & & & 9 & 3 \\
\hline 16 & $\begin{array}{l}\text { AMOX/K CLAV--HYDROMORPHON-- } \\
\text { NEXIUM }\end{array}$ & & & 9 & & & 9 & 9 \\
\hline 17 & $\begin{array}{l}\text { ALBUTEROL--QVAR-- } \\
\text { TRAMADOL_HCL }\end{array}$ & 9 & & & & & 9 & 9 \\
\hline 18 & $\begin{array}{l}\text { ALBUTEROL--AZITHROMYCIN-- } \\
\text { PREDNISOLONE }\end{array}$ & 2 & 2 & 4 & 0 & 0 & 8 & 0.727 \\
\hline 19 & $\begin{array}{l}\text { AZITHROMYCIN--PREDNISONE-- } \\
\text { VENTOLIN HFA }\end{array}$ & & 0 & 3 & 0 & 5 & 8 & 1.142 \\
\hline 20 & $\begin{array}{l}\text { HYDROCO/APAP--IBUPROFEN-- } \\
\text { OXYCOD/APAP }\end{array}$ & 2 & & & 2 & 4 & 8 & 1.6 \\
\hline 21 & $\begin{array}{l}\text { AMLODIPINE--HYDROCO/APAP-- } \\
\text { LISINOPRIL }\end{array}$ & & 2 & 6 & 0 & & 8 & 1.6 \\
\hline 22 & $\begin{array}{l}\text { ALBUTEROL--FLOVENT DISK-- } \\
\text { PREDNISONE }\end{array}$ & & 8 & 0 & 0 & & 8 & 2 \\
\hline 23 & $\begin{array}{l}\text { CARISOPRODOL--IBUPROFEN-- } \\
\text { PROMETHAZINE }\end{array}$ & & & & & 8 & 8 & 4 \\
\hline 24 & $\begin{array}{l}\text { ALPRAZOLAM--FOLIC_ACID-- } \\
\text { HYDROCO/APAP }\end{array}$ & 8 & & & & & 8 & 4 \\
\hline
\end{tabular}




\begin{tabular}{|r|l|c|c|c|c|c|c|c|}
\hline 25 & $\begin{array}{l}\text { ALBUTEROL--AMOX/K CLAV-- } \\
\text { PREDNISONE }\end{array}$ & & & & 8 & & 8 & 4 \\
\hline 26 & $\begin{array}{l}\text { ACETAMINOPHEN TAB 500 MG-- } \\
\text { ATORVASTATIN--PROPRAN/HCTZ }\end{array}$ & & 8 & & & & 8 & 8 \\
\hline 27 & $\begin{array}{l}\text { HYDROXYZ HCL--RANITIDINE-- } \\
\text { TRAZODONE }\end{array}$ & $\begin{array}{l}\text { HYDROCOD/HOM--LISINOPRIL-- } \\
\text { QUETIAPINE }\end{array}$ & 8 & & & 8 & 8 \\
\hline 29 & $\begin{array}{l}\text { ESCITALOPRAM-ONDANSETRON-- } \\
\text { OXYCODONE }\end{array}$ & 8 & & & & & 8 & 8 \\
\hline 30 & $\begin{array}{l}\text { DICLOFENAC--HYDROCHLOROT-- } \\
\text { RANITIDINE }\end{array}$ & & & 8 & & 8 & 8 \\
\hline
\end{tabular}

Table 4: Top 30 single ingredients in the dataset ranked by ER visits

\begin{tabular}{|c|l|c|c|c|c|c|c|c|c|}
\hline & \multicolumn{6}{|c|}{ Number of ER } & Occ & ER & $\begin{array}{l}\text { \#ER/ } \\
\text { \#Occ }\end{array}$ \\
\hline & Single active ingredients & $\mathbf{2 0 1 1}$ & $\mathbf{2 0 1 2}$ & $\mathbf{2 0 1 3}$ & $\mathbf{2 0 1 4}$ & Total & Total & Total & Total \\
\hline 1 & AMOXICILLIN & 201 & 255 & 253 & 261 & 227 & 4,505 & 1,197 & 0.266 \\
\hline 2 & $\begin{array}{l}\text { AZITHROMYCIN } \\
\text { DIHYDRATE }\end{array}$ & 219 & 244 & 221 & 186 & 39 & 3,233 & 909 & 0.281 \\
\hline 3 & IBUPROFEN & 113 & 141 & 141 & 149 & 152 & 1,696 & 696 & 0.410 \\
\hline 4 & ALBUTEROL SULFATE & 79 & 115 & 145 & 98 & 119 & 1,875 & 556 & 0.297 \\
\hline 5 & LISINOPRIL & 66 & 46 & 69 & 70 & 40 & 1,737 & 291 & 0.168 \\
\hline 6 & $\begin{array}{l}\text { LEVOTHYROXINE } \\
\text { SODIUM }\end{array}$ & 38 & 63 & 46 & 48 & 50 & 2,393 & 245 & 0.102 \\
\hline 7 & CEPHALEXIN & 46 & 28 & 50 & 51 & 27 & 553 & 202 & 0.365 \\
\hline 8 & PREDNISONE & 32 & 42 & 46 & 48 & 23 & 438 & 191 & 0.436 \\
\hline 9 & NAPROXEN & 31 & 26 & 40 & 34 & 44 & 499 & 175 & 0.351 \\
\hline 10 & $\begin{array}{l}\text { ONDANSETRON } \\
\text { HYDROCHLORIDE }\end{array}$ & 19 & 32 & 45 & 46 & 29 & 258 & 171 & 0.663 \\
\hline 11 & OMEPRAZOLE & 21 & 36 & 37 & 34 & 28 & 726 & 156 & 0.215 \\
\hline 12 & $\begin{array}{l}\text { OXYCODONE } \\
\text { HYDROCHLORIDE }\end{array}$ & 24 & 35 & 26 & 33 & 22 & 255 & 140 & 0.549 \\
\hline 13 & ACETAMINOPHEN & 44 & 37 & 38 & 8 & 9 & 308 & 136 & 0.442 \\
\hline 14 & $\begin{array}{l}\text { CYCLOBENZAPRINE } \\
\text { HYDROCHLORIDE }\end{array}$ & 32 & 21 & 28 & 20 & 24 & 270 & 125 & 0.463 \\
\hline 15 & $\begin{array}{l}\text { TRAMADOL } \\
\text { HYDROCHLORIDE }\end{array}$ & 12 & 27 & 19 & 32 & 33 & 293 & 123 & 0.420 \\
\hline 16 & $\begin{array}{l}\text { METFORMIN } \\
\text { HYDROCHLORIDE }\end{array}$ & 38 & 32 & 11 & 16 & 25 & 802 & 122 & 0.152 \\
\hline 17 & $\begin{array}{l}\text { PENICILLIN V } \\
\text { POTASSIUM }\end{array}$ & 25 & 19 & 23 & 36 & 18 & 328 & 121 & 0.369 \\
\hline 18 & $\begin{array}{l}\text { METHYLPHENIDATE } \\
\text { HYDROCHLORIDE }\end{array}$ & 15 & 42 & 17 & 17 & 18 & 930 & 109 & 0.117 \\
\hline 19 & $\begin{array}{l}\text { RANITIDINE } \\
\text { HYDROCHLORIDE }\end{array}$ & 39 & 14 & 21 & 14 & 343 & 108 & 0.315 \\
\hline
\end{tabular}




\begin{tabular}{|c|l|c|c|c|c|c|c|c|c|}
\hline 20 & SIMVASTATIN & 20 & 25 & 20 & 22 & 20 & 1,022 & 107 & 0.105 \\
\hline 21 & $\begin{array}{l}\text { SERTRALINE } \\
\text { HYDROCHLORIDE }\end{array}$ & 32 & 15 & 24 & 23 & 12 & 603 & 106 & 0.176 \\
\hline 22 & HYDROCHLOROTHIAZIDE & 17 & 20 & 22 & 17 & 24 & 527 & 100 & 0.190 \\
\hline 23 & $\begin{array}{l}\text { CIPROFLOXACIN } \\
\text { HYDROCHLORIDE }\end{array}$ & 15 & 21 & 24 & 16 & 24 & 305 & 100 & 0.328 \\
\hline 24 & $\begin{array}{l}\text { PROMETHAZINE } \\
\text { HYDROCHLORIDE }\end{array}$ & 31 & 21 & 22 & 12 & 11 & 200 & 97 & 0.485 \\
\hline 25 & MONTELUKAST SODIUM & 16 & 27 & 13 & 12 & 27 & 452 & 95 & 0.210 \\
\hline 26 & AMLODIPINE BESYLATE & 5 & 13 & 33 & 22 & 18 & 722 & 91 & 0.126 \\
\hline 27 & $\begin{array}{l}\text { CITALOPRAM } \\
\text { HYDROBROMIDE }\end{array}$ & 24 & 17 & 25 & 15 & 9 & 459 & 90 & 0.196 \\
\hline 28 & INSULIN HUMAN & 18 & 15 & 20 & 20 & 16 & 309 & 89 & 0.288 \\
\hline 29 & ALPRAZOLAM & 19 & 19 & 10 & 22 & 19 & 246 & 89 & 0.362 \\
\hline 30 & $\begin{array}{l}\text { FLUTICASONE } \\
\text { PROPIONATE }\end{array}$ & 16 & 16 & 15 & 22 & 17 & 627 & 86 & 0.137 \\
\hline
\end{tabular}

Table 5: Top 30 two-ingredient polypharmacy ranked by ER visits

\begin{tabular}{|l|l|l|l|l|l|l|l|l|l|}
\hline & \multicolumn{2}{|l|}{ Number of ER } & \multicolumn{2}{l|}{} & Occ & ER & $\begin{array}{l}\text { \#ER } \\
\text { /\#Occ }\end{array}$ \\
\hline & $\begin{array}{l}\text { Polypharmacy of two- } \\
\text { ingredients }\end{array}$ & 2011 & 2012 & 2013 & 2014 & 2015 & Total & Total & Total \\
\hline 1 & $\begin{array}{l}\text { ACETAMINOPHEN-- } \\
\text { HYDROCODONE BITARTRATE }\end{array}$ & 121 & 98 & 99 & 180 & 130 & 1119 & 628 & 0.561 \\
\hline 2 & $\begin{array}{l}\text { ACETAMINOPHEN-- } \\
\text { CODEINE PHOSPHATE }\end{array}$ & 71 & 68 & 83 & 48 & 47 & 632 & 317 & 0.502 \\
\hline 3 & $\begin{array}{l}\text { AMOXICILLIN-- } \\
\text { CLAVULANATE POTASSIUM }\end{array}$ & 45 & 34 & 46 & 36 & 92 & 678 & 253 & 0.373 \\
\hline 4 & $\begin{array}{l}\text { ACETAMINOPHEN-- } \\
\text { OXYCODONE HYDROCHLORIDE }\end{array}$ & 49 & 45 & 42 & 42 & 57 & 440 & 235 & 0.534 \\
\hline 5 & $\begin{array}{l}\text { SULFAMETHOXAZOLE-- } \\
\text { TRIMETHOPRIM }\end{array}$ & 31 & 42 & 35 & 19 & 62 & 508 & 189 & 0.372 \\
\hline 6 & $\begin{array}{l}\text { AMOXICILLIN-- } \\
\text { IBUPROFEN }\end{array}$ & 8 & 23 & 14 & 13 & 34 & 735 & 92 & 0.125 \\
\hline 7 & $\begin{array}{l}\text { HYDROCHLOROTHIAZIDE-- } \\
\text { LISINOPRIL }\end{array}$ & 10 & 14 & 18 & 16 & 9 & 75 & 67 & 0.893 \\
\hline 8 & $\begin{array}{l}\text { ALBUTEROL SULFATE-- } \\
\text { PREDNISONE }\end{array}$ & 13 & 9 & 7 & 13 & 18 & 103 & 60 & 0.583 \\
\hline 9 & $\begin{array}{l}\text { AMOXICILLIN-- } \\
\text { ALBUTEROL SULFATE }\end{array}$ & 8 & 17 & 12 & 7 & 12 & 190 & 56 & 0.295 \\
\hline 10 & $\begin{array}{l}\text { ALBUTEROL SULFATE-- } \\
\text { FLUTICASONE PROPIONATE }\end{array}$ & $\begin{array}{l}\text { CYCLOBENZAPRINE } \\
\text { HYDROCHLORIDE-- } \\
\text { IBUPROFEN }\end{array}$ & 16 & 11 & 8 & 13 & 98 & 56 & 0.571 \\
\hline
\end{tabular}




\begin{tabular}{|c|c|c|c|c|c|c|c|c|c|}
\hline 12 & $\begin{array}{l}\text { AZITHROMYCIN DIHYDRATE-- } \\
\text { IBUPROFEN }\end{array}$ & 8 & 13 & 8 & 15 & 3 & 122 & 47 & 0.385 \\
\hline 13 & IBUPROFEN--PREDNISONE & 1 & 0 & 3 & 42 & 0 & 14 & 46 & 3.286 \\
\hline 14 & AMLODIPINE BESYLATE--LISINOPRIL & 4 & 5 & 1 & 16 & 17 & 147 & 43 & 0.293 \\
\hline 15 & $\begin{array}{l}\text { ETHINYL ESTRADIOL-- } \\
\text { NORETHINDRONE ACETATE }\end{array}$ & 2 & 13 & 7 & 2 & 12 & 311 & 36 & 0.116 \\
\hline 16 & $\begin{array}{l}\text { AZITHROMYCIN DIHYDRATE-- } \\
\text { PREDNISONE }\end{array}$ & 5 & 7 & 12 & 5 & 7 & 63 & 36 & 0.571 \\
\hline 17 & $\begin{array}{l}\text { ALBUTEROL SULFATE-- } \\
\text { BUDESONIDE }\end{array}$ & 4 & 13 & 6 & 7 & 3 & 100 & 33 & 0.330 \\
\hline 18 & $\begin{array}{l}\text { CYCLOBENZAPRINE } \\
\text { HYDROCHLORIDE--NAPROXEN }\end{array}$ & 8 & 7 & 10 & 6 & 2 & 74 & 33 & 0.446 \\
\hline 19 & $\begin{array}{l}\text { IBUPROFEN--TRAMADOL } \\
\text { HYDROCHLORIDE }\end{array}$ & 2 & 9 & 3 & 8 & 11 & 31 & 33 & 1.065 \\
\hline 20 & $\begin{array}{l}\text { AMOXICILLIN--AZITHROMYCIN } \\
\text { DIHYDRATE }\end{array}$ & 15 & 2 & 5 & 6 & 4 & 63 & 32 & 0.508 \\
\hline 21 & $\begin{array}{l}\text { SERTRALINE HYDROCHLORIDE-- } \\
\text { TRAZODONE HYDROCHLORIDE }\end{array}$ & 1 & 7 & 10 & 13 & 0 & 30 & 31 & 1.033 \\
\hline 22 & $\begin{array}{l}\text { HYDROCODONE BITARTRATE-- } \\
\text { HOMATROPINE METHYLBROMIDE }\end{array}$ & 11 & 7 & 11 & 1 & 0 & 94 & 30 & 0.319 \\
\hline 23 & LISINOPRIL--SIMVASTATIN & 10 & 6 & 3 & 5 & 5 & 222 & 29 & 0.131 \\
\hline 24 & ATENOLOL--CHLORTHALIDONE & 8 & 8 & 3 & 9 & 1 & 145 & 29 & 0.200 \\
\hline 25 & $\begin{array}{l}\text { ALBUTEROL SULFATE-- } \\
\text { MONTELUKAST SODIUM }\end{array}$ & 12 & 7 & 6 & 2 & 2 & 138 & 29 & 0.210 \\
\hline 26 & $\begin{array}{l}\text { ALBUTEROL SULFATE-- } \\
\text { PREDNISOLONE }\end{array}$ & 7 & 9 & 4 & 7 & 2 & 52 & 29 & 0.558 \\
\hline 27 & $\begin{array}{l}\text { ETONOGESTREL--ETHINYL } \\
\text { ESTRADIOL }\end{array}$ & 10 & 5 & 5 & 3 & 5 & 248 & 28 & 0.113 \\
\hline 28 & $\begin{array}{l}\text { ALBUTEROL SULFATE-- } \\
\text { BECLOMETHASONE } \\
\text { DIPROPIONATE }\end{array}$ & 4 & 9 & 5 & 3 & 7 & 104 & 28 & 0.269 \\
\hline 29 & $\begin{array}{l}\text { AMLODIPINE BESYLATE-- } \\
\text { HYDROCHLOROTHIAZIDE }\end{array}$ & 1 & 6 & 6 & 8 & 5 & 113 & 26 & 0.230 \\
\hline 30 & AMOXICILLIN--PREDNISONE & 4 & 1 & 5 & 15 & 1 & 49 & 26 & 0.530 \\
\hline
\end{tabular}


Table 6: Top 30 three-ingredient polypharmacy ranked by ER visits.

\begin{tabular}{|c|c|c|c|c|c|c|c|c|}
\hline & & Numb & er of $E$ & & & & ER & \#ER/ \\
\hline & Polypharmacy of three-ingredients & 2011 & 2012 & 2013 & 2014 & 2015 & Total & Total \\
\hline 1 & $\begin{array}{l}\text { ACETAMINOPHEN--HYDROCODONE } \\
\text { BITARTRATE--IBUPROFEN }\end{array}$ & 8 & 23 & 15 & 57 & 34 & 137 & 0.840 \\
\hline 2 & $\begin{array}{l}\text { ACETAMINOPHEN--IBUPROFEN-- } \\
\text { OXYCODONE HYDROCHLORIDE }\end{array}$ & 7 & 11 & 15 & 13 & 7 & 53 & 0.654 \\
\hline 3 & $\begin{array}{l}\text { ACETAMINOPHEN-- } \\
\text { CYCLOBENZAPRINE } \\
\text { HYDROCHLORIDE--HYDROCODONE } \\
\text { BITARTRATE }\end{array}$ & 12 & 8 & 2 & 16 & 14 & 52 & 0.684 \\
\hline 4 & $\begin{array}{l}\text { ALBUTEROL SULFATE--FLUTICASONE } \\
\text { PROPIONATE--SALMETEROL } \\
\text { XINAFOATE }\end{array}$ & 10 & 8 & 10 & 13 & 6 & 47 & 0.285 \\
\hline 5 & $\begin{array}{l}\text { ACETAMINOPHEN--HYDROCODONE } \\
\text { BITARTRATE-- } \\
\text { OXYCODONE HYDROCHLORIDE }\end{array}$ & 6 & 8 & 14 & 10 & 2 & 40 & 0.727 \\
\hline 6 & $\begin{array}{l}\text { ACETAMINOPHEN--CODEINE } \\
\text { PHOSPHATE--IBUPROFEN }\end{array}$ & 9 & 0 & 6 & 10 & 13 & 38 & 0.760 \\
\hline 7 & $\begin{array}{l}\text { ACETAMINOPHEN--HYDROCODONE } \\
\text { BITARTRATE--PROMETHAZINE } \\
\text { HYDROCHLORIDE }\end{array}$ & 4 & 4 & 9 & 14 & 3 & 34 & 1.478 \\
\hline 8 & $\begin{array}{l}\text { AZITHROMYCIN DIHYDRATE-- } \\
\text { ACETAMINOPHEN-- } \\
\text { CODEINE PHOSPHATE }\end{array}$ & 5 & 11 & 11 & 5 & 0 & 32 & 0.444 \\
\hline 9 & $\begin{array}{l}\text { AZITHROMYCIN DIHYDRATE-- } \\
\text { ACETAMINOPHEN-- } \\
\text { HYDROCODONE BITARTRATE }\end{array}$ & 5 & 12 & 4 & 9 & 1 & 31 & 0.660 \\
\hline 10 & $\begin{array}{l}\text { ACETAMINOPHEN--BUTALBITAL-- } \\
\text { CAFFEINE }\end{array}$ & 3 & 5 & 5 & 9 & 8 & 30 & 0.476 \\
\hline 11 & $\begin{array}{l}\text { HYDROCORTISONE--NEOMYCIN } \\
\text { SULFATE-- } \\
\text { POLYMYXIN B SULFATE }\end{array}$ & 5 & 1 & 14 & 10 & 0 & 30 & 0.244 \\
\hline 12 & $\begin{array}{l}\text { ACETAMINOPHEN--AZITHROMYCIN } \\
\text { DIHYDRATE--CODEINE PHOSPHATE }\end{array}$ & 3 & 8 & 10 & 7 & & 28 & 0.571 \\
\hline 13 & $\begin{array}{l}\text { ACETAMINOPHEN--CLINDAMYCIN } \\
\text { PHOSPHATE-- } \\
\text { HYDROCODONE BITARTRATE }\end{array}$ & 0 & 2 & 4 & 11 & 11 & 28 & 0.875 \\
\hline 14 & $\begin{array}{l}\text { ACETAMINOPHEN--HYDROCODONE } \\
\text { BITARTRATE--PENICILLIN V } \\
\text { POTASSIUM }\end{array}$ & 6 & 2 & 5 & 6 & 6 & 25 & 0.455 \\
\hline 15 & $\begin{array}{l}\text { AMLODIPINE BESYLATE-- } \\
\text { HYDROCHLOROTHIAZIDE-- } \\
\text { LOSARTAN POTASSIUM }\end{array}$ & 0 & 11 & 4 & 2 & 8 & 25 & 0.357 \\
\hline
\end{tabular}




\begin{tabular}{|c|c|c|c|c|c|c|c|c|}
\hline 16 & $\begin{array}{l}\text { AMOXICILLIN--ACETAMINOPHEN-- } \\
\text { HYDROCODONE BITARTRATE }\end{array}$ & 5 & 0 & 2 & 9 & 8 & 24 & 0.264 \\
\hline 17 & $\begin{array}{l}\text { AMOXICILLIN--CLAVULANATE } \\
\text { POTASSIUM--IBUPROFEN }\end{array}$ & 4 & 1 & 3 & 12 & 4 & 24 & 0.828 \\
\hline 18 & $\begin{array}{l}\text { ATENOLOL--CARISOPRODOL-- } \\
\text { HYDROCHLOROTHIAZIDE }\end{array}$ & & & 24 & & & 24 & 24.00 \\
\hline 19 & $\begin{array}{l}\text { AMLODIPINE BESYLATE-- } \\
\text { HYDROCHLOROTHIAZIDE--LISINOPRIL }\end{array}$ & 4 & 1 & 9 & 8 & 1 & 23 & 0.228 \\
\hline 20 & $\begin{array}{l}\text { ACETAMINOPHEN--AMOXICILLIN-- } \\
\text { HYDROCODONE BITARTRATE }\end{array}$ & 4 & 3 & 6 & 4 & 5 & 22 & 0.449 \\
\hline 21 & $\begin{array}{l}\text { ACETAMINOPHEN--AZITHROMYCIN } \\
\text { DIHYDRATE-- } \\
\text { HYDROCODONE BITARTRATE }\end{array}$ & 4 & 3 & 2 & 6 & 7 & 22 & 0.710 \\
\hline 22 & $\begin{array}{l}\text { ACETAMINOPHEN--HYDROCODONE } \\
\text { BITARTRATE--NAPROXEN }\end{array}$ & 2 & 4 & 4 & 8 & 4 & 22 & 0.688 \\
\hline 23 & $\begin{array}{l}\text { BROMPHENIRAMINE MALEATE-- } \\
\text { DEXTROMETHORPHAN } \\
\text { HYDROBROMIDE-- } \\
\text { PSEUDOEPHEDRINE } \\
\text { HYDROCHLORIDE } \\
\end{array}$ & 0 & 5 & 8 & 5 & 4 & 22 & 0.142 \\
\hline 24 & $\begin{array}{l}\text { INSULIN GLARGINE--IBUPROFEN-- } \\
\text { OMEPRAZOLE }\end{array}$ & & 10 & 11 & & & 21 & 10.500 \\
\hline 25 & $\begin{array}{l}\text { ACETAMINOPHEN--CEPHALEXIN-- } \\
\text { HYDROCODONE BITARTRATE }\end{array}$ & 7 & 6 & 1 & 4 & 0 & 18 & 0.375 \\
\hline 26 & $\begin{array}{l}\text { ACETAMINOPHEN--CIPROFLOXACIN } \\
\text { HYDROCHLORIDE--OXYCODONE } \\
\text { HYDROCHLORIDE }\end{array}$ & & 7 & 2 & 5 & 4 & 18 & 2.571 \\
\hline 27 & $\begin{array}{l}\text { ACETAMINOPHEN-- } \\
\text { CYCLOBENZAPRINE } \\
\text { HYDROCHLORIDE--OXYCODONE } \\
\text { HYDROCHLORIDE }\end{array}$ & 1 & 4 & 9 & 4 & 0 & 18 & 1.059 \\
\hline 28 & $\begin{array}{l}\text { DIVALPROEX SODIUM-- } \\
\text { LEVETIRACETAM } \\
-- \\
\text { OXCARBAZEPINE }\end{array}$ & & 6 & 12 & & & 18 & 3.600 \\
\hline 29 & $\begin{array}{l}\text { ACETAMINOPHEN--AZITHROMYCIN } \\
\text { DIHYDRATE-- } \\
\text { OXYCODONE HYDROCHLORIDE }\end{array}$ & 8 & 1 & 2 & 3 & 2 & 16 & 0.762 \\
\hline 30 & $\begin{array}{l}\text { ALBUTEROL SULFATE--FLUTICASONE } \\
\text { PROPIONATE--MONTELUKAST } \\
\text { SODIUM }\end{array}$ & 1 & 1 & 11 & 0 & 3 & 16 & 0.347 \\
\hline
\end{tabular}


Table 7: Single TC1-class polypharmacy in the dataset ranked by ER visits

\begin{tabular}{|c|c|c|c|c|c|c|c|c|}
\hline & \multirow[b]{2}{*}{ Single TC1 Classes } & \multicolumn{5}{|c|}{ Number of ER } & \multirow{2}{*}{$\begin{array}{l}\text { ER } \\
\text { Total }\end{array}$} & \multirow[b]{2}{*}{$\begin{array}{l}\text { \#ER/ } \\
\text { \#Occ }\end{array}$} \\
\hline & & 2011 & 2012 & 2013 & 2014 & 2015 & & \\
\hline 1 & Central nervous system agents & 1,278 & 1,375 & 1,141 & 1,538 & 1,460 & 6,792 & 0.396 \\
\hline 2 & Anti-infectives & 825 & 790 & 980 & 807 & 651 & 4,053 & 0.3 \\
\hline 3 & Cardiovascular agents & 379 & 361 & 692 & 404 & 409 & 2,245 & 0.206 \\
\hline 4 & Respiratory agents & 307 & 340 & 442 & 254 & 304 & 1,647 & 0.257 \\
\hline 5 & Topical agents & 213 & 210 & 511 & 248 & 310 & 1,492 & 0.226 \\
\hline 6 & Hormones/hormone modifiers & 217 & 262 & 571 & 235 & 206 & 1,491 & 0.17 \\
\hline 7 & Metabolic agents & 172 & 174 & 400 & 153 & 150 & 1,049 & 0.177 \\
\hline 8 & Psychotherapeutic agents & 150 & 145 & 267 & 165 & 104 & 831 & 0.246 \\
\hline 9 & Gastrointestinal agents & 101 & 160 & 183 & 152 & 118 & 714 & 0.279 \\
\hline 10 & Nutritional products & 161 & 117 & 178 & 137 & 76 & 669 & 0.296 \\
\hline 11 & Coagulation modifiers & 30 & 11 & 16 & 30 & 32 & 119 & 0.358 \\
\hline 12 & Antineoplastics & 14 & 23 & 46 & 10 & 17 & 110 & 0.212 \\
\hline 13 & Miscellaneous agents & 18 & 8 & 49 & 20 & 10 & 105 & 0.248 \\
\hline 14 & Genitourinary tract agents & 3 & 3 & 16 & 2 & 3 & 27 & 0.157 \\
\hline 15 & Immunologic agents & 2 & 5 & 4 & 10 & 2 & 23 & 0.168 \\
\hline 16 & Alternative medicines & 1 & 0 & 3 & 14 & 2 & 20 & 0.351 \\
\hline \multirow[t]{2}{*}{17} & Biologicals & 0 & 0 & 0 & 0 & 0 & 0 & 0 \\
\hline & Grand Total & 3,910 & 4,024 & 5,526 & 4,244 & 3,880 & 21,584 & 0.27 \\
\hline
\end{tabular}


Table 8: Top 30 two TC1-class polypharmacy ranked by ER visits.

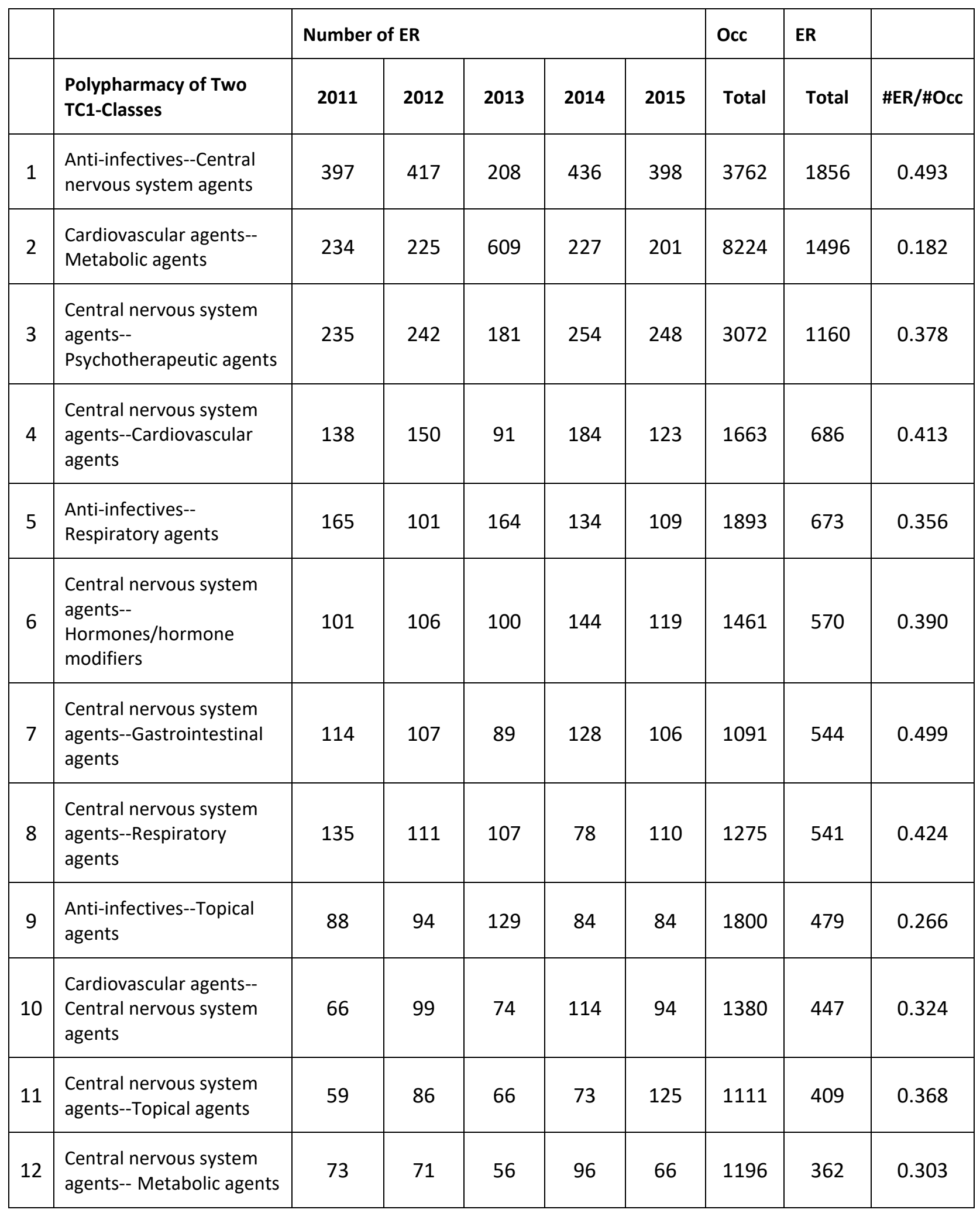




\begin{tabular}{|c|c|c|c|c|c|c|c|c|c|}
\hline 13 & $\begin{array}{l}\text { Hormones/hormone } \\
\text { modifiers--Respiratory } \\
\text { agents }\end{array}$ & 64 & 83 & 59 & 73 & 47 & 835 & 326 & 0.390 \\
\hline 14 & $\begin{array}{l}\text { Respiratory agents-- } \\
\text { Topical agents }\end{array}$ & 39 & 40 & 130 & 69 & 39 & 1197 & 317 & 0.265 \\
\hline 15 & $\begin{array}{l}\text { Anti-infectives-- } \\
\text { Hormones/hormone } \\
\text { modifiers }\end{array}$ & 52 & 50 & 76 & 73 & 65 & 1075 & 316 & 0.294 \\
\hline 16 & $\begin{array}{l}\text { Central nervous system } \\
\text { agents--Nutritional } \\
\text { products }\end{array}$ & 78 & 62 & 63 & 45 & 55 & 602 & 303 & 0.503 \\
\hline 17 & $\begin{array}{l}\text { Cardiovascular agents-- } \\
\text { Gastrointestinal agents }\end{array}$ & 18 & 38 & 79 & 50 & 51 & 830 & 236 & 0.284 \\
\hline 18 & $\begin{array}{l}\text { Anti-infectives-- } \\
\text { Cardiovascular agents }\end{array}$ & 33 & 47 & 48 & 47 & 46 & 736 & 221 & 0.300 \\
\hline 19 & $\begin{array}{l}\text { Cardiovascular agents-- } \\
\text { Hormones/hormone } \\
\text { modifiers }\end{array}$ & 25 & 40 & 59 & 42 & 48 & 1335 & 214 & 0.160 \\
\hline 20 & $\begin{array}{l}\text { Cardiovascular agents-- } \\
\text { Psychotherapeutic agents }\end{array}$ & 27 & 57 & 39 & 26 & 36 & 752 & 185 & 0.246 \\
\hline 21 & $\begin{array}{l}\text { Cardiovascular agents-- } \\
\text { Nutritional products }\end{array}$ & 28 & 39 & 38 & 40 & 34 & 501 & 179 & 0.357 \\
\hline 22 & $\begin{array}{l}\text { Cardiovascular agents-- } \\
\text { Topical agents }\end{array}$ & 34 & 15 & 50 & 37 & 31 & 651 & 167 & 0.257 \\
\hline 23 & $\begin{array}{l}\text { Hormones/hormone } \\
\text { modifiers--Topical agents }\end{array}$ & 19 & 24 & 49 & 30 & 16 & 631 & 138 & 0.219 \\
\hline 24 & $\begin{array}{l}\text { Cardiovascular agents-- } \\
\text { Respiratory agents }\end{array}$ & 26 & 26 & 32 & 25 & 22 & 504 & 131 & 0.260 \\
\hline 25 & $\begin{array}{l}\text { Anti-infectives-- } \\
\text { Metabolic agents }\end{array}$ & 17 & 20 & 33 & 23 & 24 & 347 & 117 & 0.337 \\
\hline 26 & $\begin{array}{l}\text { Anti-infectives-- } \\
\text { Nutritional products }\end{array}$ & 25 & 27 & 18 & 30 & 14 & 277 & 114 & 0.412 \\
\hline 27 & $\begin{array}{l}\text { Anti-infectives-- } \\
\text { Gastrointestinal agents }\end{array}$ & 10 & 24 & 13 & 28 & 37 & 322 & 112 & 0.348 \\
\hline
\end{tabular}




\begin{tabular}{|c|l|c|c|c|c|c|c|c|c|}
\hline 28 & $\begin{array}{l}\text { Hormones/hormone } \\
\text { modifiers-- } \\
\text { Psychotherapeutic agents }\end{array}$ & 18 & 24 & 39 & 14 & 13 & 577 & 108 & 0.187 \\
\hline 29 & $\begin{array}{l}\text { Hormones/hormone } \\
\text { modifiers--Nutritional } \\
\text { products }\end{array}$ & 15 & 16 & 37 & 4 & 25 & 184 & 97 & 0.527 \\
\hline 30 & $\begin{array}{l}\text { Gastrointestinal } \\
\text { agents-Nutritional } \\
\text { products }\end{array}$ & 13 & 28 & 28 & 18 & 144 & 95 & 0.660 \\
\hline
\end{tabular}

Table 9: Top 30 three TC1-class polypharmacy ranked by ER visits.

\begin{tabular}{|c|c|c|c|c|c|c|c|c|c|}
\hline & & Number & f ER & & & & Occ & ER & \\
\hline & $\begin{array}{l}\text { Polypharmacy of Three TC1- } \\
\text { Classes }\end{array}$ & 2011 & 2012 & 2013 & 2014 & 2015 & Total & Total & \#ER/\#Occ \\
\hline 1 & $\begin{array}{l}\text { Cardiovascular agents--Central } \\
\text { nervous system agents-- } \\
\text { Metabolic agents }\end{array}$ & 52 & 83 & 108 & 84 & 62 & 1376 & 389 & 0.283 \\
\hline 2 & $\begin{array}{l}\text { Central nervous system agents-- } \\
\text { Cardiovascular agents-- } \\
\text { Metabolic agents }\end{array}$ & 57 & 82 & 80 & 109 & 53 & 1157 & 381 & 0.329 \\
\hline 3 & $\begin{array}{l}\text { Central nervous system agents-- } \\
\text { Cardiovascular agents-- } \\
\text { Psychotherapeutic agents }\end{array}$ & 48 & 55 & 41 & 70 & 93 & 648 & 307 & 0.474 \\
\hline 4 & $\begin{array}{l}\text { Anti-infectives--Central nervous } \\
\text { system agents-- } \\
\text { Psychotherapeutic agents }\end{array}$ & 110 & 54 & 24 & 76 & 33 & 409 & 297 & 0.726 \\
\hline 5 & $\begin{array}{l}\text { Anti-infectives--Central nervous } \\
\text { system agents--Topical agents }\end{array}$ & 49 & 42 & 50 & 54 & 62 & 489 & 257 & 0.526 \\
\hline 6 & $\begin{array}{l}\text { Anti-infectives--Central nervous } \\
\text { system agents--Respiratory } \\
\text { agents }\end{array}$ & 47 & 54 & 52 & 50 & 50 & 540 & 253 & 0.469 \\
\hline 7 & $\begin{array}{l}\text { Anti-infectives-- } \\
\text { Hormones/hormone modifiers-- } \\
\text { Respiratory agents }\end{array}$ & 34 & 23 & 53 & 60 & 47 & 1038 & 217 & 0.209 \\
\hline 8 & $\begin{array}{l}\text { Cardiovascular agents-- } \\
\text { Gastrointestinal agents-- } \\
\text { Metabolic agents }\end{array}$ & 48 & 50 & 21 & 52 & 46 & 473 & 217 & 0.459 \\
\hline 9 & $\begin{array}{l}\text { Anti-infectives--Central nervous } \\
\text { system agents--Gastrointestinal } \\
\text { agents }\end{array}$ & 35 & 36 & 49 & 37 & 57 & 282 & 214 & 0.759 \\
\hline 10 & $\begin{array}{l}\text { Anti-infectives--Central nervous } \\
\text { system agents-- } \\
\text { Hormones/hormone modifiers }\end{array}$ & 59 & 29 & 44 & 38 & 40 & 439 & 210 & 0.478 \\
\hline
\end{tabular}




\begin{tabular}{|c|c|c|c|c|c|c|c|c|c|}
\hline 11 & $\begin{array}{l}\text { Cardiovascular agents-- } \\
\text { Coagulation modifiers-- } \\
\text { Metabolic agents }\end{array}$ & 31 & 50 & 41 & 43 & 32 & 525 & 197 & 0.375 \\
\hline 12 & $\begin{array}{l}\text { Cardiovascular agents-- } \\
\text { Hormones/hormone modifiers-- } \\
\text { Metabolic agents }\end{array}$ & 44 & 41 & 40 & 37 & 32 & 1159 & 194 & 0.167 \\
\hline 13 & $\begin{array}{l}\text { Central nervous system agents-- } \\
\text { Gastrointestinal agents-- } \\
\text { Psychotherapeutic agents }\end{array}$ & 30 & 32 & 21 & 74 & 31 & 307 & 188 & 0.612 \\
\hline 14 & $\begin{array}{l}\text { Anti-infectives--Central nervous } \\
\text { system agents--Cardiovascular } \\
\text { agents }\end{array}$ & 44 & 38 & 21 & 34 & 43 & 283 & 180 & 0.636 \\
\hline 15 & $\begin{array}{l}\text { Central nervous system agents-- } \\
\text { Hormones/hormone modifiers-- } \\
\text { Psychotherapeutic agents }\end{array}$ & 30 & 29 & 27 & 29 & 51 & 473 & 166 & 0.351 \\
\hline 16 & $\begin{array}{l}\text { Cardiovascular agents--Central } \\
\text { nervous system agents-- } \\
\text { Psychotherapeutic agents }\end{array}$ & 27 & 32 & 29 & 26 & 34 & 386 & 148 & 0.383 \\
\hline 17 & $\begin{array}{l}\text { Central nervous system agents-- } \\
\text { Psychotherapeutic agents-- } \\
\text { Respiratory agents }\end{array}$ & 25 & 12 & 24 & 45 & 41 & 271 & 147 & 0.542 \\
\hline 18 & $\begin{array}{l}\text { Coagulation modifiers-- } \\
\text { Cardiovascular agents-- } \\
\text { Metabolic agents }\end{array}$ & 24 & 36 & 43 & 15 & 25 & 375 & 143 & 0.381 \\
\hline 19 & $\begin{array}{l}\text { Cardiovascular agents--Central } \\
\text { nervous system agents-- } \\
\text { Gastrointestinal agents } \\
\end{array}$ & 25 & 36 & 35 & 32 & 10 & 228 & 138 & 0.605 \\
\hline 20 & $\begin{array}{l}\text { Anti-infectives--Respiratory } \\
\text { agents--Topical agents }\end{array}$ & 28 & 20 & 24 & 27 & 31 & 445 & 130 & 0.292 \\
\hline 21 & $\begin{array}{l}\text { Anti-infectives--Cardiovascular } \\
\text { agents-- Metabolic agents }\end{array}$ & 22 & 20 & 39 & 22 & 25 & 490 & 128 & 0.261 \\
\hline 22 & $\begin{array}{l}\text { Central nervous system agents-- } \\
\text { Cardiovascular agents-- } \\
\text { Gastrointestinal agents }\end{array}$ & 36 & 15 & 14 & 32 & 24 & 305 & 121 & 0.397 \\
\hline 23 & $\begin{array}{l}\text { Cardiovascular agents-- } \\
\text { Metabolic agents--Nutritional } \\
\text { products }\end{array}$ & 15 & 19 & 29 & 30 & 24 & 622 & 117 & 0.188 \\
\hline 24 & $\begin{array}{l}\text { Central nervous system agents-- } \\
\text { Metabolic agents -- } \\
\text { Psychotherapeutic agents }\end{array}$ & 12 & 15 & 32 & 36 & 20 & 319 & 115 & 0.361 \\
\hline 25 & $\begin{array}{l}\text { Cardiovascular agents-- } \\
\text { Metabolic agents --Topical } \\
\text { agents }\end{array}$ & 13 & 25 & 37 & 22 & 16 & 652 & 113 & 0.173 \\
\hline 26 & $\begin{array}{l}\text { Cardiovascular agents-- } \\
\text { Metabolic agents -- } \\
\text { Psychotherapeutic agents }\end{array}$ & 9 & 19 & 36 & 12 & 27 & 567 & 103 & 0.182 \\
\hline 27 & $\begin{array}{l}\text { Central nervous system agents-- } \\
\text { Respiratory agents--Topical } \\
\text { agents }\end{array}$ & 17 & 24 & 17 & 13 & 32 & 310 & 103 & 0.332 \\
\hline 28 & $\begin{array}{l}\text { Anti-infectives--Central nervous } \\
\text { system agents-- Metabolic } \\
\text { agents }\end{array}$ & 19 & 29 & 6 & 18 & 19 & 208 & 91 & 0.438 \\
\hline
\end{tabular}




\begin{tabular}{|c|l|c|c|c|c|c|c|c|c|}
\hline 29 & $\begin{array}{l}\text { Central nervous system agents-- } \\
\text { Gastrointestinal agents- } \\
\text { Metabolic agents }\end{array}$ & 16 & 16 & 31 & 16 & 11 & 204 & 90 & 0.441 \\
\hline $\begin{array}{l}\text { Anti-infectives-- } \\
\text { Cardiovascular agents- } \\
\begin{array}{l}\text { Central nervous system } \\
\text { agents }\end{array}\end{array}$ & 20 & 9 & 4 & 34 & 18 & 158 & 85 & 0.538 \\
\hline
\end{tabular}

Table 10: Top 30 single TC1S1-class in the dataset ranked by ER visits.

\begin{tabular}{|c|c|c|c|c|c|c|c|c|}
\hline & & \multicolumn{5}{|c|}{ Number of ER } & \multirow{2}{*}{$\begin{array}{c}\text { ER } \\
\text { Total }\end{array}$} & \multirow[b]{2}{*}{ \#ER/\#Oc } \\
\hline & Single TC1S1-Classes & 2011 & 2012 & 2013 & 2014 & 2015 & & \\
\hline 1 & ANALGESICS & 752 & 802 & 781 & 877 & 761 & 3,973 & 0.466 \\
\hline 2 & PENICILLINS & 287 & 317 & 335 & 336 & 316 & 1,591 & 0.284 \\
\hline 3 & MACROLIDE_DERIVATIVES & 230 & 254 & 223 & 186 & 39 & 932 & 0.278 \\
\hline 4 & BRONCHODILATORS & 127 & 152 & 173 & 125 & 146 & 723 & 0.269 \\
\hline 5 & DERMATOLOGICAL_AGENTS & 123 & 129 & 139 & 131 & 185 & 707 & 0.213 \\
\hline 6 & ANTIDEPRESSANTS & 117 & 121 & 131 & 118 & 88 & 575 & 0.206 \\
\hline 7 & SEX_HORMONES & 119 & 131 & 108 & 100 & 101 & 559 & 0.113 \\
\hline 8 & ANTICONVULSANTS & 85 & 115 & 131 & 89 & 85 & 505 & 0.395 \\
\hline 9 & ANTIEMETIC/ANTIVERTIGO_AGENTS & 73 & 90 & 91 & 86 & 80 & 420 & 0.580 \\
\hline 10 & ANTIDIABETIC_AGENTS & 103 & 92 & 70 & 70 & 71 & 406 & 0.195 \\
\hline 11 & IRON_PRODUCTS & 102 & 81 & 95 & 85 & 31 & 394 & 0.389 \\
\hline 12 & CNS_STIMULANTS & 51 & 94 & 80 & 75 & 87 & 387 & 0.131 \\
\hline 13 & $\begin{array}{l}\text { ANGIOTENSIN_CONVERTING } \\
\text { _ENZYME_INHIBITORS }\end{array}$ & 76 & 49 & 87 & 75 & 42 & 329 & 0.164 \\
\hline 14 & PROTON_PUMP_INHIBITORS & 56 & 74 & 73 & 61 & 51 & 315 & 0.220 \\
\hline 15 & ADRENAL_CORTICAL_STEROIDS & 52 & 58 & 77 & 73 & 42 & 302 & 0.387 \\
\hline 16 & CEPHALOSPORINS & 67 & 45 & 66 & 70 & 42 & 290 & 0.333 \\
\hline 17 & OPHTHALMIC_PREPARATIONS & 34 & 52 & 85 & 55 & 64 & 290 & 0.167 \\
\hline 18 & ANTIHYPERLIPIDEMIC_AGENTS & 50 & 58 & 54 & 58 & 62 & 282 & 0.096 \\
\hline 19 & THYROID_HORMONES & 40 & 65 & 48 & 50 & 55 & 258 & 0.101 \\
\hline 20 & ANTIHYPERTENSIVE_COMBINATIONS & 53 & 44 & 40 & 56 & 49 & 242 & 0.126 \\
\hline 21 & MISCELLANEOUS_ANTIBIOTICS & 33 & 45 & 44 & 21 & 60 & 203 & 0.373 \\
\hline 22 & BETA-ADRENERGIC_BLOCKING_AGENTS & 40 & 47 & 37 & 35 & 37 & 196 & 0.178 \\
\hline 23 & MUSCLE_RELAXANTS & 39 & 26 & 36 & 35 & 30 & 166 & 0.376 \\
\hline 24 & ANTIHISTAMINES & 29 & 37 & 28 & 36 & 34 & 164 & 0.178 \\
\hline 25 & DIURETICS & 31 & 32 & 34 & 31 & 36 & 164 & 0.229 \\
\hline 26 & ANTIVIRAL_AGENTS & 39 & 23 & 28 & 34 & 36 & 160 & 0.216 \\
\hline 27 & ANXIOLYTICSSEDATIVESAND_HYPNOTICS & 30 & 33 & 23 & 45 & 29 & 160 & 0.251 \\
\hline 28 & (H2_ANTAGONISTS) & 27 & 51 & 24 & 28 & 20 & 150 & 0.351 \\
\hline 29 & UPPER_RESPIRATORY_COMBINATIONS & 39 & 31 & 44 & 20 & 16 & 150 & 0.182 \\
\hline 30 & QUINOLONES & 27 & 26 & 40 & 28 & 20 & 141 & 0.359 \\
\hline
\end{tabular}


Table 11: Top 30 two TC1S1-class polypharmacy ranked by ER visits.

\begin{tabular}{|c|c|c|c|c|c|c|c|c|c|}
\hline & & Num & er of $E$ & & & & Occ & ER & \\
\hline & Polypharmacy of Two TC1S1-Classes & 2011 & 2012 & 2013 & 2014 & 2015 & Total & Total & \#ER/\#Occ \\
\hline 1 & ANALGESICS--MUSCLE_RELAXANTS & 94 & 116 & 105 & 117 & 93 & 921 & 525 & 0.57 \\
\hline 2 & ANALGESICS--PENICILLINS & 88 & 81 & 139 & 106 & 105 & 1,169 & 519 & 0.44 \\
\hline 3 & ANALGESICS--MACROLIDE_DERIVATIVES & 70 & 95 & 74 & 77 & 19 & 609 & 335 & 0.55 \\
\hline 4 & $\begin{array}{l}\text { ANALGESICS-- } \\
\text { ANTIEMETIC/ANTIVERTIGO_AGENTS }\end{array}$ & 26 & 23 & 50 & 58 & 51 & 176 & 208 & 1.18 \\
\hline 5 & $\begin{array}{l}\text { ADRENAL_CORTICAL_STEROIDS-- } \\
\text { BRONCHODILATORS }\end{array}$ & 30 & 58 & 43 & 39 & 22 & 319 & 192 & 0.60 \\
\hline 6 & ANALGESICS--ANTICONVULSANTS & 19 & 37 & 17 & 37 & 52 & 281 & 162 & 0.58 \\
\hline 7 & $\begin{array}{l}\text { ANALGESICS-- } \\
\text { DERMATOLOGICAL_AGENTS }\end{array}$ & 25 & 33 & 24 & 14 & 64 & 322 & 160 & 0.50 \\
\hline 8 & ANALGESICS--CEPHALOSPORINS & 36 & 17 & 18 & 38 & 14 & 223 & 123 & 0.55 \\
\hline 9 & $\begin{array}{l}\text { ANALGESICS-- } \\
\text { PROTON_PUMP_INHIBITORS }\end{array}$ & 26 & 30 & 23 & 14 & 21 & 260 & 114 & 0.44 \\
\hline 10 & ANALGESICS--IRON_PRODUCTS & 24 & 20 & 33 & 22 & 8 & 157 & 107 & 0.68 \\
\hline 11 & $\begin{array}{l}\text { ANALGESICS-- } \\
\text { MISCELLANEOUS_ANTIBIOTICS }\end{array}$ & 20 & 13 & 21 & 6 & 42 & 114 & 102 & 0.89 \\
\hline 12 & ANALGESICS--SEX_HORMONES & 22 & 20 & 13 & 26 & 20 & 278 & 101 & 0.36 \\
\hline 13 & BRONCHODILATORS--PENICILLINS & 21 & 19 & 14 & 23 & 23 & 210 & 100 & 0.48 \\
\hline 14 & $\begin{array}{l}\text { BRONCHODILATORS-- } \\
\text { RESPIRATORY_INHALANT_PRODUCTS }\end{array}$ & 17 & 29 & 18 & 11 & 18 & 308 & 93 & 0.30 \\
\hline 15 & ANALGESICS--BRONCHODILATORS & 32 & 8 & 13 & 15 & 22 & 149 & 90 & 0.60 \\
\hline 16 & ANALGESICS--ANTIDEPRESSANTS & 12 & 16 & 30 & 20 & 10 & 200 & 88 & 0.44 \\
\hline 17 & $\begin{array}{l}\text { ANTIEMETIC/ANTIVERTIGO_AGENTS-- } \\
\text { ANALGESICS }\end{array}$ & 8 & 6 & 32 & 9 & 33 & 89 & 88 & 0.99 \\
\hline 18 & ANALGESICS--QUINOLONES & 13 & 16 & 22 & 8 & 23 & 96 & 82 & 0.85 \\
\hline 19 & MACROLIDE_DERIVATIVES--PENICILLINS & 32 & 4 & 19 & 16 & 9 & 148 & 80 & 0.54 \\
\hline 20 & $\begin{array}{l}\text { ADRENAL_CORTICAL_STEROIDS-- } \\
\text { ANALGESICS }\end{array}$ & 6 & 9 & 9 & 50 & 5 & 80 & 79 & 0.99 \\
\hline 21 & ANTIDEPRESSANTS--ANTICONVULSANTS & 15 & 20 & 7 & 10 & 24 & 192 & 76 & 0.40 \\
\hline 22 & $\begin{array}{l}\text { ANALGESICS-- } \\
\text { LINCOMYCIN_DERIVATIVES }\end{array}$ & 7 & 14 & 11 & 28 & 15 & 100 & 75 & 0.75 \\
\hline 23 & ANTICONVULSANTS--ANALGESICS & 10 & 15 & 10 & 11 & 27 & 165 & 73 & 0.44 \\
\hline 24 & ANALGESICS--LAXATIVES & 9 & 7 & 21 & 23 & 10 & 142 & 70 & 0.49 \\
\hline 25 & $\begin{array}{l}\text { ANALGESICS-- } \\
\text { ANTIHYPERTENSIVE_COMBINATIONS }\end{array}$ & 11 & 12 & 13 & 13 & 18 & 120 & 67 & 0.56 \\
\hline 26 & ANALGESICS--DIURETICS & 21 & 23 & 6 & 5 & 11 & 101 & 66 & 0.65 \\
\hline 27 & $\begin{array}{l}\text { ANALGESICS-- } \\
\text { BETA_ADRENERGIC_BLOCKING_AGENTS }\end{array}$ & 18 & 17 & 18 & 7 & 5 & 138 & 65 & 0.47 \\
\hline 28 & $\begin{array}{l}\text { BRONCHODILATORS-- } \\
\text { LEUKOTRIENE_MODIFIERS }\end{array}$ & 29 & 10 & 12 & 6 & 5 & 263 & 62 & 0.24 \\
\hline 29 & $\begin{array}{l}\text { BRONCHODILATORS-- } \\
\text { MACROLIDE_DERIVATIVES }\end{array}$ & 20 & 13 & 5 & 17 & 7 & 175 & 62 & 0.35 \\
\hline 30 & $\begin{array}{l}\text { ANALGESICS-- } \\
\text { ADRENAL CORTICAL STEROIDS }\end{array}$ & 10 & 17 & 9 & 8 & 18 & 99 & 62 & 0.63 \\
\hline
\end{tabular}


Table 12: Top 30 three TC1S1-class polypharmacy ranked by ER visits.

\begin{tabular}{|c|c|c|c|c|c|c|c|c|c|}
\hline & & Numb & er of ER & & & & Occ & ER & \\
\hline & Polypharmacy of Three TC1S1-Classes & 2011 & 2012 & 2013 & 2014 & 2015 & Total & Total & $\begin{array}{l}\text { \#ER } \\
\text { /\#Occ }\end{array}$ \\
\hline 1 & $\begin{array}{l}\text { ADRENAL_CORTICAL_STEROIDS--BRONCHODILATORS-- } \\
\text { MACROLIDE_DERIVATIVES }\end{array}$ & 11 & 13 & 7 & 9 & 12 & 77 & 52 & 0.68 \\
\hline 2 & ANALGESICS--ANTICONVULSANTS--MUSCLE_RELAXANTS & 12 & 8 & 5 & 9 & 11 & 66 & 45 & 0.68 \\
\hline 3 & $\begin{array}{l}\text { ANALGESICS--ANTIDEPRESSANTS-- } \\
\text { ANXIOLYTICSSEDATIVESAND_HYPNOTICS }\end{array}$ & 27 & 5 & 4 & 7 & 0 & 23 & 43 & 1.87 \\
\hline 4 & $\begin{array}{l}\text { ADRENAL_CORTICAL_STEROIDS--BRONCHODILATORS-- } \\
\text { PENICILLINS }\end{array}$ & 7 & 6 & 15 & 7 & 2 & 56 & 37 & 0.66 \\
\hline 5 & $\begin{array}{l}\text { ANALGESICS--PENICILLINS-- } \\
\text { UPPER_RESPIRATORY_COMBINATIONS }\end{array}$ & 7 & 9 & 11 & 3 & 0 & 66 & 30 & 0.45 \\
\hline 6 & $\begin{array}{l}\text { ANTIDIABETIC_AGENTS-- } \\
\text { ANGIOTENSIN_CONVERTING_ENZYME_INHIBITORS-- } \\
\text { ANTIHYPERLIPIDEMIC_AGENTS }\end{array}$ & 5 & 10 & 7 & 4 & 3 & 185 & 29 & 0.16 \\
\hline 7 & ANALGESICS--ANTICONVULSANTS--ANTIDEPRESSANTS & 7 & 3 & 7 & 1 & 10 & 33 & 28 & 0.85 \\
\hline 8 & $\begin{array}{l}\text { ANTIDIABETIC_AGENTS-- } \\
\text { ANGIOTENSIN_CONVERTING_ENZYME_INHIBITORS-- } \\
\text { BETA--ADRENERGIC_BLOCKING_AGENTS }\end{array}$ & 5 & 8 & 2 & 10 & 1 & 43 & 26 & 0.60 \\
\hline 9 & $\begin{array}{l}\text { BRONCHODILATORS--LEUKOTRIENE_MODIFIERS-- } \\
\text { RESPIRATORY_INHALANT_PRODUCTS }\end{array}$ & 6 & 1 & 15 & 3 & 1 & 62 & 26 & 0.42 \\
\hline 10 & ANALGESICS--IRON_PRODUCTS--LAXATIVES & 6 & 4 & 8 & 7 & 0 & 31 & 25 & 0.81 \\
\hline 11 & ANALGESICS--ANTIDEPRESSANTS--MUSCLE_RELAXANTS & 2 & 7 & 4 & 5 & 6 & 46 & 24 & 0.52 \\
\hline 12 & $\begin{array}{l}\text { BETA--ADRENERGIC_BLOCKING_AGENTS--DIURETICS-- } \\
\text { MUSCLE_RELAXANTS }\end{array}$ & & & 24 & 0 & & 2 & 24 & 12.00 \\
\hline 13 & $\begin{array}{l}\text { ANALGESICS--ANTIEMETIC/ANTIVERTIGO_AGENTS-- } \\
\text { MUSCLE_RELAXANTS }\end{array}$ & 9 & & 2 & 9 & 3 & 15 & 23 & 1.53 \\
\hline 14 & ANALGESICS--MACROLIDE_DERIVATIVES--PENICILLINS & 8 & 4 & 9 & 1 & 1 & 30 & 23 & 0.77 \\
\hline 15 & $\begin{array}{l}\text { ANALGESICS--MUSCLE_RELAXANTS-- } \\
\text { PROTON_PUMP_INHIBITORS }\end{array}$ & 5 & 9 & 2 & 1 & 6 & 41 & 23 & 0.56 \\
\hline 16 & $\begin{array}{l}\text { ANGIOTENSIN_CONVERTING_ENZYME_INHIBITORS-- } \\
\text { BETA--ADRENERGIC_BLOCKING_AGENTS--DIURETICS }\end{array}$ & 1 & 4 & 3 & 9 & 6 & 86 & 23 & 0.27 \\
\hline 17 & ANALGESICS--ANTIDEPRESSANTS--ANTICONVULSANTS & 1 & 7 & 6 & 3 & 5 & 39 & 22 & 0.56 \\
\hline 18 & ANALGESICS--MUSCLE_RELAXANTS--PENICILLINS & 1 & 3 & 8 & 9 & 1 & 21 & 22 & 1.05 \\
\hline 19 & $\begin{array}{l}\text { ANTIDIABETIC_AGENTS--ANALGESICS-- } \\
\text { PROTON_PUMP_INHIBITORS }\end{array}$ & 0 & 10 & 11 & 0 & 0 & 9 & 21 & 2.33 \\
\hline 20 & $\begin{array}{l}\text { ANTIHYPERTENSIVE_COMBINATIONS--BETA-- } \\
\text { ADRENERGIC_BLOCKING_AGENTS-- } \\
\text { CALCIUM_CHANNEL_BLOCKING_AGENTS }\end{array}$ & 0 & 4 & 15 & 0 & 2 & 44 & 21 & 0.48 \\
\hline 21 & $\begin{array}{l}\text { BETA_ADRENERGIC_BLOCKING_AGENTS-- } \\
\text { CALCIUM_CHANNEL_BLOCKING_AGENTS--DIURETICS }\end{array}$ & 2 & 3 & 0 & 11 & 5 & 40 & 21 & 0.53 \\
\hline 22 & $\begin{array}{l}\text { ADRENAL_CORTICAL_STEROIDS--BRONCHODILATORS-- } \\
\text { RESPIRATORY_INHALANT_PRODUCTS }\end{array}$ & 3 & 8 & 2 & 2 & 5 & 29 & 20 & 0.69 \\
\hline 23 & $\begin{array}{l}\text { ANALGESICS--ANTIEMETIC/ANTIVERTIGO_AGENTS-- } \\
\text { PENICILLINS }\end{array}$ & 1 & 8 & 3 & 4 & 4 & 24 & 20 & 0.83 \\
\hline 24 & ANALGESICS--OTIC_PREPARATIONS--PENICILLINS & 7 & 1 & 1 & 5 & 6 & 21 & 20 & 0.95 \\
\hline 25 & $\begin{array}{l}\text { ANALGESICS--ANTIEMETIC/ANTIVERTIGO_AGENTS-- } \\
\text { MACROLIDE_DERIVATIVES }\end{array}$ & 1 & 4 & 9 & 3 & 2 & 17 & 19 & 1.12 \\
\hline
\end{tabular}




\begin{tabular}{|c|l|c|c|c|c|c|c|c|c|}
\hline 26 & $\begin{array}{l}\text { ANALGESICS--BRONCHODILATORS-- } \\
\text { RESPIRATORY_INHALANT_PRODUCTS }\end{array}$ & 13 & 4 & 1 & 1 & 0 & 22 & 19 & 0.86 \\
\hline 27 & $\begin{array}{l}\text { ANALGESICS--CEPHALOSPORINS-- } \\
\text { MISCELLANEOUS_ANTIBIOTICS }\end{array}$ & 3 & 2 & 1 & 7 & 6 & 16 & 19 & 1.19 \\
\hline 28 & $\begin{array}{l}\text { ANTICOAGULANTS--ANTIARRHYTHMIC_AGENTS-- } \\
\text { BETA_ADRENERGIC_BLOCKING_AGENTS }\end{array}$ & & 2 & 8 & 2 & 7 & 15 & 19 & 1.27 \\
\hline 29 & $\begin{array}{l}\text { ADRENAL_CORTICAL_STEROIDS--BRONCHODILATORS-- } \\
\text { QUINOLONES }\end{array}$ & 0 & 7 & 1 & 5 & 4 & 15 & 17 & 1.13 \\
\hline 30 & ANALGESICS-IRON_PRODUCTS--VITAMINS & & 7 & 4 & 1 & 5 & 16 & 17 & 1.06 \\
\hline
\end{tabular}




\section{Richness of three-elements polypharmacy}

Table 13: Top 30 drug-triples and their individual and co-occurrences probability, and occurrence richness ranked by ER total.

\begin{tabular}{|c|c|c|c|c|c|c|c|c|c|c|}
\hline & Drug triples & Occ & $\begin{array}{l}\text { part1- } \\
\text { occ }\end{array}$ & $\begin{array}{l}\text { part2- } \\
\text { occ }\end{array}$ & $\begin{array}{l}\text { part3- } \\
\text { occ }\end{array}$ & $\begin{array}{l}\text { p(three- } \\
\text { occ) }\end{array}$ & $\begin{array}{l}\text { p(part1- } \\
\text { occ) }\end{array}$ & $\begin{array}{l}p(\text { part2- } \\
o c c)\end{array}$ & $\begin{array}{l}\text { p(part3- } \\
\text { occ) }\end{array}$ & $\begin{array}{l}\text { Occ- } \\
\text { richness }\end{array}$ \\
\hline 1 & $\begin{array}{l}\text { CYCLOBENZAPR-- } \\
\text { HYDROCO/APAP-- } \\
\text { IBUPROFEN }\end{array}$ & 29 & 260 & 1238 & 1675 & 0.00113 & 0.0038 & 0.0182 & 0.0247 & 651.95 \\
\hline 2 & $\begin{array}{l}\text { ATENOLOL-- } \\
\text { CARISOPRODOL-- } \\
\text { HYDROCHLOROT }\end{array}$ & 1 & 447 & 37 & 463 & 0.00004 & 0.0066 & 0.0005 & 0.0068 & 1582.84 \\
\hline 3 & $\begin{array}{l}\text { IBUPROFEN-- } \\
\text { LANTUS-- } \\
\text { OMEPRAZOLE }\end{array}$ & 2 & 1675 & 75 & 721 & 0.00008 & 0.0247 & 0.0011 & 0.0106 & 267.64 \\
\hline 4 & $\begin{array}{l}\text { AMLODIPINE-- } \\
\text { METOPROLOL-- } \\
\text { VALSART/HCTZ }\end{array}$ & 5 & 684 & 184 & 34 & 0.00019 & 0.0101 & 0.0027 & 0.0005 & 14162.66 \\
\hline 5 & $\begin{array}{l}\text { DIVALPROEX-- } \\
\text { LEVETIRACETA-- } \\
\text { OXCARBAZEPIN }\end{array}$ & 3 & 46 & 104 & 72 & 0.00012 & 0.0007 & 0.0015 & 0.0011 & 105566.29 \\
\hline 6 & $\begin{array}{l}\text { CLINDAMYCIN-- } \\
\text { CYCLOBENZAPR-- } \\
\text { OXYCODONE }\end{array}$ & 1 & 260 & 260 & 211 & 0.00004 & 0.0038 & 0.0038 & 0.0031 & 849.76 \\
\hline 7 & $\begin{array}{l}\text { GLIPIZIDE-- } \\
\text { LISINOPRIL-- } \\
\text { METFORMIN }\end{array}$ & 33 & 38 & 1737 & 780 & 0.00128 & 0.0006 & 0.0256 & 0.0115 & 7768.97 \\
\hline 8 & $\begin{array}{l}\text { ALBUTEROL-- } \\
\text { AMOXICILLIN-- } \\
\text { PREDNISONE }\end{array}$ & 5 & 785 & 4471 & 436 & 0.00019 & 0.0116 & 0.0659 & 0.0064 & 39.60 \\
\hline 9 & $\begin{array}{l}\text { AMOXICILLIN-- } \\
\text { HYDROCO/APAP-- } \\
\text { IBUPROFEN }\end{array}$ & 20 & 4471 & 1238 & 1675 & 0.00078 & 0.0659 & 0.0182 & 0.0247 & 26.15 \\
\hline 10 & $\begin{array}{l}\text { APAP/CODEINE-- } \\
\text { AZITHROMYCIN-- } \\
\text { IBUPROFEN }\end{array}$ & 6 & 614 & 3180 & 1675 & 0.00023 & 0.0090 & 0.0469 & 0.0247 & 22.24 \\
\hline 11 & $\begin{array}{l}\text { CEPHALEXIN-- } \\
\text { HYDROCO/APAP-- } \\
\text { IBUPROFEN }\end{array}$ & 5 & 538 & 1238 & 1675 & 0.00019 & 0.0079 & 0.0182 & 0.0247 & 54.32 \\
\hline 12 & $\begin{array}{l}\text { MIRTAZAPINE-- } \\
\text { PRAVASTATIN-- } \\
\text { TERAZOSIN }\end{array}$ & 3 & 28 & 257 & 22 & 0.00012 & 0.0004 & 0.0038 & 0.0003 & 229686.31 \\
\hline 13 & $\begin{array}{l}\text { LISINOPRIL-- } \\
\text { PERCOCET-- } \\
\text { SIMVASTATIN }\end{array}$ & 1 & 1737 & 62 & 1014 & 0.00004 & 0.0256 & 0.0009 & 0.0149 & 110.99 \\
\hline 14 & $\begin{array}{l}\text { AMOXICILLIN-- } \\
\text { APAP/CODEINE-- } \\
\text { IBUPROFEN }\end{array}$ & 9 & 4471 & 614 & 1675 & 0.00035 & 0.0659 & 0.0090 & 0.0247 & 23.72 \\
\hline
\end{tabular}




\begin{tabular}{|c|c|c|c|c|c|c|c|c|c|c|}
\hline 15 & $\begin{array}{l}\text { FEXOFENADINE-- } \\
\text { LEVETIRACETA-- } \\
\text { VITAMIN D }\end{array}$ & 3 & 23 & 104 & 217 & 0.00012 & 0.0003 & 0.0015 & 0.0032 & 70053.21 \\
\hline 16 & $\begin{array}{l}\text { AMOX/K CLAV-- } \\
\text { HYDROMORPHON- } \\
\text {-NEXIUM }\end{array}$ & 1 & 485 & 14 & 186 & 0.00004 & 0.0071 & 0.0002 & 0.0027 & 9597.21 \\
\hline 17 & $\begin{array}{l}\text { ALBUTEROL-- } \\
\text { QVAR-- } \\
\text { TRAMADOL_HCL } \\
\end{array}$ & 1 & 785 & 61 & 41 & 0.00004 & 0.0116 & 0.0009 & 0.0006 & 6173.68 \\
\hline 18 & $\begin{array}{l}\text { ALBUTEROL-- } \\
\text { AZITHROMYCIN-- } \\
\text { PREDNISOLONE } \\
\end{array}$ & 11 & 785 & 3180 & 150 & 0.00043 & 0.0116 & 0.0469 & 0.0022 & 356.07 \\
\hline 19 & $\begin{array}{l}\text { AZITHROMYCIN-- } \\
\text { PREDNISONE-- } \\
\text { VENTOLIN HFA }\end{array}$ & 7 & 3180 & 436 & 318 & 0.00027 & 0.0469 & 0.0064 & 0.0047 & 192.44 \\
\hline 20 & $\begin{array}{l}\text { HYDROCO/APAP-- } \\
\text { IBUPROFEN-- } \\
\text { OXYCOD/APAP }\end{array}$ & 5 & 1238 & 1675 & 324 & 0.00019 & 0.0182 & 0.0247 & 0.0048 & 90.20 \\
\hline 21 & $\begin{array}{l}\text { AMLODIPINE-- } \\
\text { HYDROCO/APAP-- } \\
\text { LISINOPRIL }\end{array}$ & 5 & 684 & 1238 & 1737 & 0.00019 & 0.0101 & 0.0182 & 0.0256 & 41.20 \\
\hline 22 & $\begin{array}{l}\text { ALBUTEROL-- } \\
\text { FLOVENT DISK-- } \\
\text { PREDNISONE } \\
\end{array}$ & 4 & 785 & 31 & 436 & 0.00016 & 0.0116 & 0.0005 & 0.0064 & 4569.51 \\
\hline 23 & $\begin{array}{l}\text { CARISOPRODOL-- } \\
\text { IBUPROFEN-- } \\
\text { PROMETHAZINE }\end{array}$ & 2 & 37 & 1675 & 187 & 0.00008 & 0.0005 & 0.0247 & 0.0028 & 2091.70 \\
\hline 24 & $\begin{array}{l}\text { ALPRAZOLAM-- } \\
\text { FOLIC_ACID-- } \\
\text { HYDROCO/APAP }\end{array}$ & 2 & 227 & 12 & 1238 & 0.00008 & 0.0033 & 0.0002 & 0.0182 & 7188.36 \\
\hline 25 & $\begin{array}{l}\text { ALBUTEROL-- } \\
\text { AMOX/K CLAV-- } \\
\text { PREDNISONE } \\
\end{array}$ & 2 & 785 & 485 & 436 & 0.00008 & 0.0116 & 0.0071 & 0.0064 & 146.04 \\
\hline 26 & $\begin{array}{l}\text { ACETAMINOPHEN } \\
\text { TAB } 500 \text { MG-- } \\
\text { ATORVASTATIN-- } \\
\text { PROPRAN/HCTZ } \\
\end{array}$ & 2 & 26 & 556 & 126 & 0.00008 & 0.0004 & 0.0082 & 0.0019 & 13308.80 \\
\hline 27 & $\begin{array}{l}\text { HYDROXYZ HCL-- } \\
\text { RANITIDINE-- } \\
\text { TRAZODONE } \\
\end{array}$ & 1 & 54 & 327 & 80 & 0.00004 & 0.0008 & 0.0048 & 0.0012 & 8580.18 \\
\hline 28 & $\begin{array}{l}\text { HYDROCOD/HOM- } \\
\text {-LISINOPRIL-- } \\
\text { QUETIAPINE }\end{array}$ & 1 & 57 & 1737 & 29 & 0.00004 & 0.0008 & 0.0256 & 0.0004 & 4221.39 \\
\hline 29 & $\begin{array}{l}\text { ESCITALOPRAM-- } \\
\text { ONDANSETRON-- } \\
\text { OXYCODONE }\end{array}$ & 1 & 197 & 254 & 211 & 0.00004 & 0.0029 & 0.0037 & 0.0031 & 1148.01 \\
\hline 30 & $\begin{array}{l}\text { DICLOFENAC-- } \\
\text { HYDROCHLOROT-- } \\
\text { RANITIDINE }\end{array}$ & 1 & 116 & 463 & 327 & 0.00004 & 0.0017 & 0.0068 & 0.0048 & 690.15 \\
\hline
\end{tabular}


Table 14: Individual and joint probability of occurrence for the top 30 two- drug polypharmacy (sorted by ER total) with richness of ER.

\begin{tabular}{|c|c|c|c|c|c|c|c|c|c|c|}
\hline & Drug triples & ER & $\begin{array}{l}\text { part1- } \\
\text { EROcc }\end{array}$ & $\begin{array}{l}\text { part2- } \\
\text { EROcc }\end{array}$ & $\begin{array}{l}\text { part3- } \\
\text { EROcc }\end{array}$ & $\begin{array}{c}\text { p(part1- } \\
\text { ERocc) }\end{array}$ & $\begin{array}{c}\text { p(part2- } \\
\text { ERocc) }\end{array}$ & $\begin{array}{l}\text { p(part } \\
3- \\
\text { ERocc) }\end{array}$ & $\begin{array}{l}\text { p(three- } \\
\text { case-ER) }\end{array}$ & ER-richness \\
\hline 1 & $\begin{array}{l}\text { CYCLOBENZAPR-- } \\
\text { HYDROCO/APAP-- } \\
\text { IBUPROFEN }\end{array}$ & 27 & 122 & 698 & 691 & 0.0076 & 0.0436 & 0.0431 & 0.0033 & 229.43 \\
\hline 2 & $\begin{array}{l}\text { ATENOLOL-- } \\
\text { CARISOPRODOL-- } \\
\text { HYDROCHLOROT }\end{array}$ & 24 & 66 & 3 & 85 & 0.0041 & 0.0002 & 0.0053 & 0.0029 & 713024.60 \\
\hline 3 & $\begin{array}{l}\text { IBUPROFEN-- } \\
\text { LANTUS-- } \\
\text { OMEPRAZOLE }\end{array}$ & 21 & 691 & 18 & 154 & 0.0431 & 0.0011 & 0.0096 & 0.0026 & 5481.83 \\
\hline 4 & $\begin{array}{l}\text { AMLODIPINE-- } \\
\text { METOPROLOL-- } \\
\text { VALSART/HCTZ }\end{array}$ & 12 & 81 & 36 & 6 & 0.0051 & 0.0022 & 0.0004 & 0.0015 & 342941.36 \\
\hline 5 & $\begin{array}{l}\text { DIVALPROEX-- } \\
\text { LEVETIRACETA-- } \\
\text { OXCARBAZEPIN }\end{array}$ & 12 & 29 & 54 & 11 & 0.0018 & 0.0034 & 0.0007 & 0.0015 & 348316.62 \\
\hline 6 & $\begin{array}{l}\text { CLINDAMYCIN-- } \\
\text { CYCLOBENZAPR-- } \\
\text { OXYCODONE }\end{array}$ & 12 & 77 & 122 & 115 & 0.0048 & 0.0076 & 0.0072 & 0.0015 & 5554.06 \\
\hline 7 & $\begin{array}{l}\text { GLIPIZIDE-- } \\
\text { LISINOPRIL-- } \\
\text { METFORMIN } \\
\end{array}$ & 11 & 3 & 291 & 114 & 0.0002 & 0.0182 & 0.0071 & 0.0013 & 55265.10 \\
\hline 8 & $\begin{array}{l}\text { ALBUTEROL-- } \\
\text { AMOXICILLIN-- } \\
\text { PREDNISONE }\end{array}$ & 11 & 275 & 1190 & 190 & 0.0172 & 0.0743 & 0.0119 & 0.0013 & 88.46 \\
\hline 9 & $\begin{array}{l}\text { AMOXICILLIN-- } \\
\text { HYDROCO/APAP-- } \\
\text { IBUPROFEN }\end{array}$ & 10 & 1190 & 698 & 691 & 0.0743 & 0.0436 & 0.0431 & 0.0012 & 8.71 \\
\hline 10 & $\begin{array}{l}\text { APAP/CODEINE-- } \\
\text { AZITHROMYCIN-- } \\
\text { IBUPROFEN }\end{array}$ & 10 & 304 & 891 & 691 & 0.0190 & 0.0556 & 0.0431 & 0.0012 & 26.71 \\
\hline 11 & $\begin{array}{l}\text { CEPHALEXIN-- } \\
\text { HYDROCO/APAP-- } \\
\text { IBUPROFEN }\end{array}$ & 10 & 196 & 698 & 691 & 0.0122 & 0.0436 & 0.0431 & 0.0012 & 52.89 \\
\hline 12 & $\begin{array}{l}\text { MIRTAZAPINE-- } \\
\text { PRAVASTATIN-- } \\
\text { TERAZOSIN }\end{array}$ & 10 & 3 & 30 & 4 & 0.0002 & 0.0019 & 0.0002 & 0.0012 & $\begin{array}{c}13889125 . \\
08\end{array}$ \\
\hline 13 & $\begin{array}{l}\text { LISINOPRIL-- } \\
\text { PERCOCET-- } \\
\text { SIMVASTATIN } \\
\end{array}$ & 10 & 291 & 21 & 106 & 0.0182 & 0.0013 & 0.0066 & 0.0012 & 7718.97 \\
\hline 14 & $\begin{array}{l}\text { AMOXICILLIN-- } \\
\text { APAP/CODEINE-- } \\
\text { IBUPROFEN }\end{array}$ & 9 & 1190 & 304 & 691 & 0.0743 & 0.0190 & 0.0431 & 0.0011 & 18.00 \\
\hline 15 & $\begin{array}{l}\text { FEXOFENADINE-- } \\
\text { LEVETIRACETA-- } \\
\text { VITAMIN D }\end{array}$ & 9 & 3 & 54 & 26 & 0.0002 & 0.0034 & 0.0016 & 0.0011 & $\begin{array}{c}1068394.2 \\
4\end{array}$ \\
\hline 16 & $\begin{array}{l}\text { AMOX/K CLAV-- } \\
\text { HYDROMORPHON } \\
\text {--NEXIUM }\end{array}$ & 9 & 187 & 8 & 29 & 0.0117 & 0.0005 & 0.0018 & 0.0011 & 103726.64 \\
\hline 17 & $\begin{array}{l}\text { ALBUTEROL-- } \\
\text { QVAR-- } \\
\text { TRAMADOL_HCL }\end{array}$ & 9 & 275 & 17 & 12 & 0.0172 & 0.0011 & 0.0007 & 0.0011 & 80215.27 \\
\hline 18 & $\begin{array}{l}\text { ALBUTEROL-- } \\
\text { AZITHROMYCIN-- } \\
\text { PREDNISOLONE }\end{array}$ & 8 & 275 & 891 & 54 & 0.0172 & 0.0556 & 0.0034 & 0.0010 & 302.32 \\
\hline 19 & $\begin{array}{l}\text { AZITHROMYCIN-- } \\
\text { PREDNISONE-- } \\
\text { VENTOLIN HFA }\end{array}$ & 8 & 891 & 190 & 75 & 0.0556 & 0.0119 & 0.0047 & 0.0010 & 315.05 \\
\hline
\end{tabular}




\begin{tabular}{|c|l|c|c|c|c|c|c|c|c|c|}
\hline 20 & $\begin{array}{l}\text { HYDROCO/APAP-- } \\
\text { IBUPROFEN-- } \\
\text { OXYCOD/APAP }\end{array}$ & 8 & 698 & 691 & 175 & 0.0436 & 0.0431 & 0.0109 & 0.0010 & 47.39 \\
\hline $\begin{array}{l}\text { AMLODIPINE-- } \\
\text { HYDROCO/APAP-- } \\
\text { LISINOPRIL }\end{array}$ & 8 & 81 & 698 & 291 & 0.0051 & 0.0436 & 0.0182 & 0.0010 & 243.13 \\
\hline $\begin{array}{l}\text { ALBUTEROL-- } \\
\text { FLOVENT DISK-- } \\
\text { PREDNISONE }\end{array}$ & 8 & 275 & 15 & 190 & 0.0172 & 0.0009 & 0.0119 & 0.0010 & 5103.76 \\
\hline $\begin{array}{l}\text { CARISOPRODOL-- } \\
\text { IBUPROFEN-- } \\
\text { PROMETHAZINE }\end{array}$ & 8 & 3 & 691 & 85 & 0.0002 & 0.0431 & 0.0053 & 0.0010 & 22701.22 \\
\hline $\begin{array}{l}\text { ALPRAZOLAM-- } \\
\text { FOLICAACID-- } \\
\text { HYDROCO/APAP }\end{array}$ & 8 & 81 & 3 & 698 & 0.0051 & 0.0002 & 0.0436 & 0.0010 & 23583.36 \\
\hline $\begin{array}{l}\text { ALBUTEROL-- } \\
\text { AMOX/KCLAV-- } \\
\text { PREDNISONE }\end{array}$ & 8 & 275 & 187 & 190 & 0.0172 & 0.0117 & 0.0119 & 0.0010 & 409.39 \\
\hline $\begin{array}{l}\text { ACETAMINOPHEN } \\
\text { TAB 5OO MG-- } \\
\text { ATORVASTATIN-- } \\
\text { PROPRAN/HCTZ }\end{array}$ & 8 & 13 & 45 & 20 & 0.0008 & 0.0028 & 0.0012 & 0.0010 & 341886.16 \\
\hline $\begin{array}{l}\text { HYDROXYZ HCL-- } \\
\text { RANITIDINE-- } \\
\text { TRAZODONE }\end{array}$ & 8 & 10 & 99 & 13 & 0.0006 & 0.0062 & 0.0008 & 0.0010 & 310805.60 \\
\hline 28 & $\begin{array}{l}\text { HYDROCOD/HOM- } \\
\text {-LISINOPRIL-- } \\
\text { QUETIAPINE }\end{array}$ & 8 & 16 & 291 & 11 & 0.0010 & 0.0182 & 0.0007 & 0.0010 & 78101.92 \\
\hline 29 & $\begin{array}{l}\text { ESCITALOPRAM-- } \\
\text { ONDANSETRON-- } \\
\text { OXYCODONE }\end{array}$ & 8 & 37 & 167 & 115 & 0.0023 & 0.0104 & 0.0072 & 0.0010 & 5629.26 \\
\hline $\begin{array}{l}\text { DICLOFENAC-- } \\
\text { HYDROCHLOROT-- } \\
\text { RANITIDINE }\end{array}$ & 8 & 25 & 85 & 99 & 0.0016 & 0.0053 & 0.0062 & 0.0010 & 19013.99 \\
\hline
\end{tabular}


Figure 1: Relationship between drug triples and occurrence and ER richness. Logarithms are in base 10.

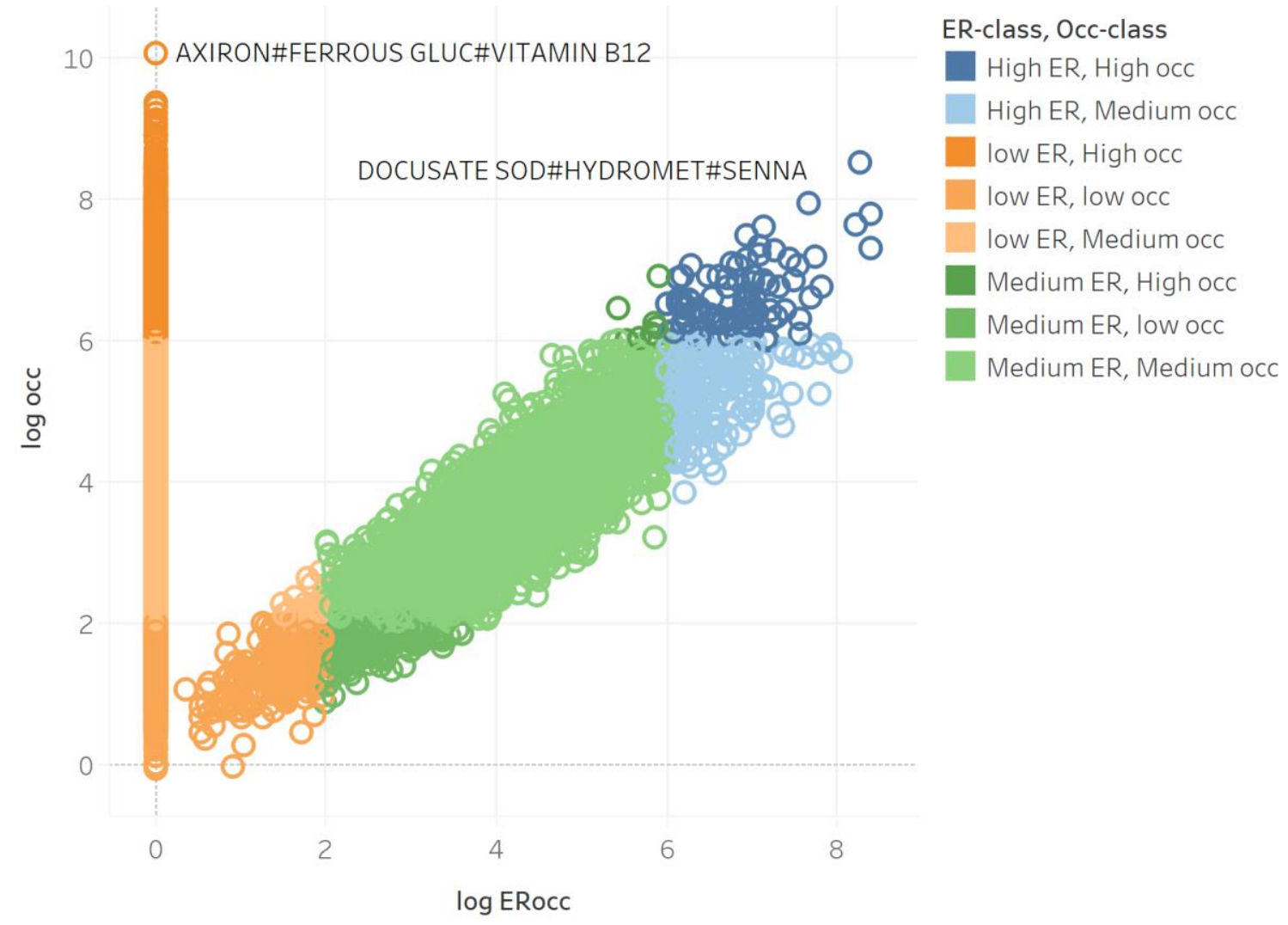

Table 15: Top 30 active ingredient-triples and their individual and co-occurrences probability, and occurrence richness ranked by ER total.

\begin{tabular}{|c|c|c|c|c|c|c|c|c|c|c|}
\hline & Active ingredient triples & Occ & $\begin{array}{l}\text { part1- } \\
\text { occ }\end{array}$ & $\begin{array}{l}\text { part2- } \\
\text { occ }\end{array}$ & $\begin{array}{l}\text { part3- } \\
\text { occ }\end{array}$ & $\begin{array}{l}\text { p(three- } \\
\text { occ) }\end{array}$ & $\begin{array}{l}\text { p(part1- } \\
\text { occ) }\end{array}$ & $\begin{array}{l}\text { p(part2- } \\
\text { occ) }\end{array}$ & $\begin{array}{l}\text { p(part3- } \\
\text { occ) }\end{array}$ & $\begin{array}{c}\text { Occ- } \\
\text { richness }\end{array}$ \\
\hline 1 & $\begin{array}{l}\text { ACETAMINOPHEN-- } \\
\text { HYDROCODONE } \\
\text { BITARTRATE--IBUPROFEN }\end{array}$ & 163 & 308 & 47 & 1696 & 0.0061 & 0.0052 & 0.0008 & 0.0287 & 50648.74 \\
\hline 2 & $\begin{array}{l}\text { ACETAMINOPHEN-- } \\
\text { IBUPROFEN--OXYCODONE } \\
\text { HYDROCHLORIDE }\end{array}$ & 81 & 308 & 1696 & 255 & 0.0030 & 0.0052 & 0.0287 & 0.0043 & 4638.99 \\
\hline 3 & $\begin{array}{l}\text { ACETAMINOPHEN-- } \\
\text { CYCLOBENZAPRINE } \\
\text { HYDROCHLORIDE-- } \\
\text { HYDROCODONE } \\
\text { BITARTRATE }\end{array}$ & 76 & 308 & 270 & 47 & 0.0028 & 0.0052 & 0.0046 & 0.0008 & 148339.47 \\
\hline 4 & $\begin{array}{l}\text { ALBUTEROL SULFATE-- } \\
\text { FLUTICASONE } \\
\text { PROPIONATE-- } \\
\text { SALMETEROL XINAFOATE }\end{array}$ & 165 & 1875 & 627 & 1 & 0.0061 & 0.0318 & 0.0106 & 0.0000 & 1070706.91 \\
\hline
\end{tabular}




\begin{tabular}{|c|c|c|c|c|c|c|c|c|c|c|}
\hline 5 & $\begin{array}{l}\text { ACETAMINOPHEN-- } \\
\text { HYDROCODONE } \\
\text { BITARTRATE--OXYCODONE } \\
\text { HYDROCHLORIDE } \\
\end{array}$ & 55 & 308 & 47 & 255 & 0.0020 & 0.0052 & 0.0008 & 0.0043 & 113665.69 \\
\hline 6 & $\begin{array}{l}\text { ACETAMINOPHEN-- } \\
\text { CODEINE PHOSPHATE-- } \\
\text { IBUPROFEN }\end{array}$ & 50 & 308 & - & 1696 & 0.0019 & 0.0052 & - & 0.0287 & - \\
\hline 7 & $\begin{array}{l}\text { ACETAMINOPHEN-- } \\
\text { HYDROCODONE } \\
\text { BITARTRATE-- } \\
\text { PROMETHAZINE } \\
\text { HYDROCHLORIDE }\end{array}$ & 23 & 308 & 47 & 200 & 0.0009 & 0.0052 & 0.0008 & 0.0034 & 60604.48 \\
\hline 8 & $\begin{array}{l}\text { AZITHROMYCIN } \\
\text { DIHYDRATE-- } \\
\text { ACETAMINOPHEN-- } \\
\text { CODEINE PHOSPHATE }\end{array}$ & 72 & 3233 & 308 & - & 0.0027 & 0.0548 & 0.0052 & - & - \\
\hline 9 & $\begin{array}{l}\text { AZITHROMYCIN } \\
\text { DIHYDRATE-- } \\
\text { ACETAMINOPHEN-- } \\
\text { HYDROCODONE } \\
\text { BITARTRATE }\end{array}$ & 47 & 3233 & 308 & 47 & 0.0017 & 0.0548 & 0.0052 & 0.0008 & 7661.24 \\
\hline 10 & $\begin{array}{l}\text { ACETAMINOPHEN-- } \\
\text { BUTALBITAL--CAFFEINE }\end{array}$ & 63 & 308 & - & - & 0.0023 & 0.0052 & - & - & - \\
\hline 11 & $\begin{array}{l}\text { HYDROCORTISONE-- } \\
\text { NEOMYCIN SULFATE-- } \\
\text { POLYMYXIN B SULFATE }\end{array}$ & 123 & 286 & 4 & - & 0.0046 & 0.0048 & 0.0001 & - & - \\
\hline 12 & $\begin{array}{l}\text { ACETAMINOPHEN-- } \\
\text { AZITHROMYCIN } \\
\text { DIHYDRATE--CODEINE } \\
\text { PHOSPHATE }\end{array}$ & 49 & 308 & 3233 & - & 0.0018 & 0.0052 & 0.0548 & - & - \\
\hline 13 & $\begin{array}{l}\text { ACETAMINOPHEN-- } \\
\text { CLINDAMYCIN } \\
\text { PHOSPHATE-- } \\
\text { HYDROCODONE } \\
\text { BITARTRATE }\end{array}$ & 32 & 308 & 263 & 47 & 0.0012 & 0.0052 & 0.0045 & 0.0008 & 64121.12 \\
\hline 14 & $\begin{array}{l}\text { ACETAMINOPHEN-- } \\
\text { HYDROCODONE } \\
\text { BITARTRATE--PENICILLIN V } \\
\text { POTASSIUM }\end{array}$ & 55 & 308 & 47 & 328 & 0.0020 & 0.0052 & 0.0008 & 0.0056 & 88368.15 \\
\hline 15 & $\begin{array}{l}\text { AMLODIPINE BESYLATE--- } \\
\text { HYDROCHLOROTHIAZIDE-- } \\
\text { LOSARTAN POTASSIUM }\end{array}$ & 70 & 722 & 527 & 294 & 0.0026 & 0.0122 & 0.0089 & 0.0050 & 4773.74 \\
\hline 16 & $\begin{array}{l}\text { AMOXICILLIN-- } \\
\text { ACETAMINOPHEN-- } \\
\text { HYDROCODONE } \\
\text { BITARTRATE }\end{array}$ & 91 & 4505 & 308 & 47 & 0.0034 & 0.0764 & 0.0052 & 0.0008 & 10645.19 \\
\hline 17 & $\begin{array}{l}\text { AMOXICILLIN-- } \\
\text { CLAVULANATE } \\
\text { POTASSIUM-IBUPROFEN }\end{array}$ & 29 & 4505 & - & 1696 & 0.0011 & 0.0764 & - & 0.0287 & - \\
\hline 18 & $\begin{array}{l}\text { ATENOLOL-- } \\
\text { CARISOPRODOL-- } \\
\text { HYDROCHLOROTHIAZIDE }\end{array}$ & 1 & 447 & 37 & 527 & 0.0000 & 0.0076 & 0.0006 & 0.0089 & 875.26 \\
\hline 19 & $\begin{array}{l}\text { AMLODIPINE BESYLATE-- } \\
\text { HYDROCHLOROTHIAZIDE- } \\
\text { LISINOPRIL }\end{array}$ & 101 & 722 & 527 & 1737 & 0.0038 & 0.0122 & 0.0089 & 0.0294 & 1165.81 \\
\hline
\end{tabular}




\begin{tabular}{|c|c|c|c|c|c|c|c|c|c|c|}
\hline 20 & $\begin{array}{l}\text { ACETAMINOPHEN-- } \\
\text { AMOXICILLIN-- } \\
\text { HYDROCODONE } \\
\text { BITARTRATE }\end{array}$ & 49 & 308 & 4505 & 47 & 0.0018 & 0.0052 & 0.0764 & 0.0008 & 5732.03 \\
\hline 21 & $\begin{array}{l}\text { ACETAMINOPHEN-- } \\
\text { AZITHROMYCIN } \\
\text { DIHYDRATE-- } \\
\text { HYDROCODONE } \\
\text { BITARTRATE }\end{array}$ & 31 & 308 & 3233 & 47 & 0.0012 & 0.0052 & 0.0548 & 0.0008 & 5053.16 \\
\hline 22 & $\begin{array}{l}\text { ACETAMINOPHEN-- } \\
\text { HYDROCODONE } \\
\text { BITARTRATE--NAPROXEN }\end{array}$ & 32 & 308 & 47 & 499 & 0.0012 & 0.0052 & 0.0008 & 0.0085 & 33795.30 \\
\hline 23 & $\begin{array}{l}\text { BROMPHENIRAMINE } \\
\text { MALEATE-- } \\
\text { DEXTROMETHORPHAN } \\
\text { HYDROBROMIDE-- } \\
\text { PSEUDOEPHEDRINE } \\
\text { HYDROCHLORIDE }\end{array}$ & 155 & - & 5 & 20 & 0.0058 & - & 0.0001 & 0.0003 & - \\
\hline 24 & $\begin{array}{l}\text { INSULIN GLARGINE-- } \\
\text { IBUPROFEN--OMEPRAZOLE }\end{array}$ & 2 & 79 & 1696 & 726 & 0.0001 & 0.0013 & 0.0287 & 0.0123 & 156.85 \\
\hline 25 & $\begin{array}{l}\text { ACETAMINOPHEN-- } \\
\text { CEPHALEXIN-- } \\
\text { HYDROCODONE } \\
\text { BITARTRATE }\end{array}$ & 48 & 308 & 553 & 47 & 0.0018 & 0.0052 & 0.0094 & 0.0008 & 45742.83 \\
\hline 26 & $\begin{array}{l}\text { ACETAMINOPHEN-- } \\
\text { CIPROFLOXACIN } \\
\text { HYDROCHLORIDE-- } \\
\text { OXYCODONE } \\
\text { HYDROCHLORIDE }\end{array}$ & 7 & 308 & 305 & 255 & 0.0003 & 0.0052 & 0.0052 & 0.0043 & 2229.27 \\
\hline 27 & $\begin{array}{l}\text { ACETAMINOPHEN-- } \\
\text { CYCLOBENZAPRINE } \\
\text { HYDROCHLORIDE-- } \\
\text { OXYCODONE } \\
\text { HYDROCHLORIDE }\end{array}$ & 17 & 308 & 270 & 255 & 0.0006 & 0.0052 & 0.0046 & 0.0043 & 6115.75 \\
\hline 28 & $\begin{array}{l}\text { DIVALPROEX SODIUM-- } \\
\text { LEVETIRACETAM-- } \\
\text { OXCARBAZEPINE }\end{array}$ & 5 & 49 & 112 & 82 & 0.0002 & 0.0008 & 0.0019 & 0.0014 & 84761.28 \\
\hline 29 & $\begin{array}{l}\text { ACETAMINOPHEN-- } \\
\text { AZITHROMYCIN } \\
\text { DIHYDRATE--OXYCODONE } \\
\text { HYDROCHLORIDE }\end{array}$ & 21 & 308 & 3233 & 255 & 0.0008 & 0.0052 & 0.0548 & 0.0043 & 630.93 \\
\hline 30 & $\begin{array}{l}\text { ALBUTEROL SULFATE-- } \\
\text { FLUTICASONE } \\
\text { PROPIONATE-- } \\
\text { MONTELUKAST SODIUM }\end{array}$ & 46 & 1875 & 627 & 452 & 0.0017 & 0.0318 & 0.0106 & 0.0077 & 660.40 \\
\hline
\end{tabular}

Table 16: Individual and joint probability of occurrence for the top 30 two-active ingredients polypharmacy (sorted by ER total) with richness of ER.

\begin{tabular}{|c|l|c|c|c|c|c|c|c|c|c|}
\hline & Active ingredient triples & ER & $\begin{array}{c}\text { part1- } \\
\text { EROcc }\end{array}$ & $\begin{array}{c}\text { part2- } \\
\text { EROcc }\end{array}$ & $\begin{array}{c}\text { part3- } \\
\text { EROcc }\end{array}$ & $\begin{array}{c}\text { p(part1- } \\
\text { ERocc) }\end{array}$ & $\begin{array}{c}\text { p(part2- } \\
\text { ERocc) }\end{array}$ & $\begin{array}{c}\text { p(part3 } \\
\text {-ERocc) }\end{array}$ & $\begin{array}{c}\mathbf{p} \text { (three- } \\
\text { case-ER) }\end{array}$ & $\begin{array}{c}\text { ER- } \\
\text { richness }\end{array}$ \\
\hline 1 & $\begin{array}{l}\text { ACETAMINOPHEN-- } \\
\text { HYDROCODONE } \\
\text { BITARTRATE--IBUPROFEN }\end{array}$ & 137 & 136 & 13 & 696 & 0.0101 & 0.0010 & 0.0517 & 0.0159 & 31661.23 \\
\hline 2 & $\begin{array}{l}\text { ACETAMINOPHEN-- } \\
\text { BUUPROFEN--OXYCODONE } \\
\text { HYDROCHLORIDE }\end{array}$ & 53 & 136 & 696 & 140 & 0.0101 & 0.0517 & 0.0104 & 0.0062 & 1137.36 \\
\hline
\end{tabular}




\begin{tabular}{|c|c|c|c|c|c|c|c|c|c|c|}
\hline 3 & $\begin{array}{l}\text { ACETAMINOPHEN-- } \\
\text { CYCLOBENZAPRINE } \\
\text { HYDROCHLORIDE-- } \\
\text { HYDROCODONE } \\
\text { BITARTRATE } \\
\end{array}$ & 52 & 136 & 125 & 13 & 0.0101 & 0.0093 & 0.0010 & 0.0060 & 66912.90 \\
\hline 4 & $\begin{array}{l}\text { ALBUTEROL SULFATE-- } \\
\text { FLUTICASONE } \\
\text { PROPIONATE--SALMETEROL } \\
\text { XINAFOATE }\end{array}$ & 47 & 556 & 86 & 2 & 0.0413 & 0.0064 & 0.0001 & 0.0055 & 139763.38 \\
\hline 5 & $\begin{array}{l}\text { ACETAMINOPHEN-- } \\
\text { HYDROCODONE } \\
\text { BITARTRATE--OXYCODONE } \\
\text { HYDROCHLORIDE }\end{array}$ & 40 & 136 & 13 & 140 & 0.0101 & 0.0010 & 0.0104 & 0.0047 & 45956.66 \\
\hline 6 & $\begin{array}{l}\text { ACETAMINOPHEN-- } \\
\text { CODEINE PHOSPHATE-- } \\
\text { IBUPROFEN }\end{array}$ & 38 & 136 & - & 696 & 0.0101 & - & 0.0517 & 0.0044 & - \\
\hline 7 & $\begin{array}{l}\text { ACETAMINOPHEN-- } \\
\text { HYDROCODONE } \\
\text { BITARTRATE-- } \\
\text { PROMETHAZINE } \\
\text { HYDROCHLORIDE } \\
\end{array}$ & 34 & 136 & 13 & 97 & 0.0101 & 0.0010 & 0.0072 & 0.0040 & 56379.82 \\
\hline 8 & $\begin{array}{l}\text { AZITHROMYCIN } \\
\text { DIHYDRATE-- } \\
\text { ACETAMINOPHEN-- } \\
\text { CODEINE PHOSPHATE }\end{array}$ & 32 & 909 & 136 & - & 0.0675 & 0.0101 & - & 0.0037 & - \\
\hline 9 & $\begin{array}{l}\text { AZITHROMYCIN } \\
\text { DIHYDRATE-- } \\
\text { ACETAMINOPHEN-- } \\
\text { HYDROCODONE } \\
\text { BITARTRATE } \\
\end{array}$ & 31 & 909 & 136 & 13 & 0.0675 & 0.0101 & 0.0010 & 0.0036 & 5485.48 \\
\hline 10 & $\begin{array}{l}\text { ACETAMINOPHEN-- } \\
\text { BUTALBITAL--CAFFEINE }\end{array}$ & 30 & 136 & - & - & 0.0101 & - & - & 0.0035 & - \\
\hline 11 & $\begin{array}{l}\text { HYDROCORTISONE-- } \\
\text { NEOMYCIN SULFATE-- } \\
\text { POLYMYXIN B SULFATE }\end{array}$ & 30 & 72 & 0 & - & 0.0053 & 0.0000 & - & 0.0035 & - \\
\hline 12 & $\begin{array}{l}\text { ACETAMINOPHEN-- } \\
\text { AZITHROMYCIN } \\
\text { DIHYDRATE--CODEINE } \\
\text { PHOSPHATE }\end{array}$ & 28 & 136 & 909 & - & 0.0101 & 0.0675 & - & 0.0033 & - \\
\hline 13 & $\begin{array}{l}\text { ACETAMINOPHEN-- } \\
\text { CLINDAMYCIN PHOSPHATE- } \\
\text {-HYDROCODONE } \\
\text { BITARTRATE }\end{array}$ & 28 & 136 & 77 & 13 & 0.0101 & 0.0057 & 0.0010 & 0.0033 & 58490.30 \\
\hline 14 & $\begin{array}{l}\text { ACETAMINOPHEN-- } \\
\text { HYDROCODONE } \\
\text { BITARTRATE--PENICILLIN V } \\
\text { POTASSIUM }\end{array}$ & 25 & 136 & 13 & 121 & 0.0101 & 0.0010 & 0.0090 & 0.0029 & 33233.12 \\
\hline 15 & $\begin{array}{l}\text { AMLODIPINE BESYLATE-- } \\
\text { HYDROCHLOROTHIAZIDE-- } \\
\text { LOSARTAN POTASSIUM }\end{array}$ & 25 & 91 & 100 & 42 & 0.0068 & 0.0074 & 0.0031 & 0.0029 & 18601.51 \\
\hline 16 & $\begin{array}{l}\text { AMOXICILLIN-- } \\
\text { ACETAMINOPHEN-- } \\
\text { HYDROCODONE } \\
\text { BITARTRATE }\end{array}$ & 24 & 1197 & 136 & 13 & 0.0889 & 0.0101 & 0.0010 & 0.0028 & 3225.03 \\
\hline 17 & $\begin{array}{l}\text { AMOXICILLIN-- } \\
\text { CLAVULANATE POTASSIUM- } \\
\text {-IBUPROFEN }\end{array}$ & 24 & 1197 & - & 696 & 0.0889 & - & 0.0517 & 0.0028 & - \\
\hline 18 & $\begin{array}{l}\text { ATENOLOL-- } \\
\text { CARISOPRODOL-- } \\
\text { HYDROCHLOROTHIAZIDE }\end{array}$ & 24 & 66 & 3 & 100 & 0.0049 & 0.0002 & 0.0074 & 0.0028 & 344702.82 \\
\hline 19 & $\begin{array}{l}\text { AMLODIPINE BESYLATE-- } \\
\text { HYDROCHLOROTHIAZIDE- } \\
\text { LISINOPRIL }\end{array}$ & 23 & 91 & 100 & 291 & 0.0068 & 0.0074 & 0.0216 & 0.0027 & 2469.97 \\
\hline
\end{tabular}




\begin{tabular}{|c|c|c|c|c|c|c|c|c|c|c|}
\hline 20 & $\begin{array}{l}\text { ACETAMINOPHEN-- } \\
\text { AMOXICILLIN-- } \\
\text { HYDROCODONE } \\
\text { BITARTRATE }\end{array}$ & 22 & 136 & 1197 & 13 & 0.0101 & 0.0889 & 0.0010 & 0.0026 & 2956.28 \\
\hline 21 & $\begin{array}{l}\text { ACETAMINOPHEN-- } \\
\text { AZITHROMYCIN } \\
\text { DIHYDRATE-- } \\
\text { HYDROCODONE } \\
\text { BITARTRATE }\end{array}$ & 22 & 136 & 909 & 13 & 0.0101 & 0.0675 & 0.0010 & 0.0026 & 3892.92 \\
\hline 22 & $\begin{array}{l}\text { ACETAMINOPHEN-- } \\
\text { HYDROCODONE } \\
\text { BITARTRATE--NAPROXEN }\end{array}$ & 22 & 136 & 13 & 175 & 0.0101 & 0.0010 & 0.0130 & 0.0026 & 20220.93 \\
\hline 23 & $\begin{array}{l}\text { BROMPHENIRAMINE } \\
\text { MALEATE-- } \\
\text { DEXTROMETHORPHAN } \\
\text { HYDROBROMIDE-- } \\
\text { PSEUDOEPHEDRINE } \\
\text { HYDROCHLORIDE } \\
\end{array}$ & 22 & - & 2 & 0 & - & 0.0001 & 0.0000 & 0.0026 & - \\
\hline 24 & $\begin{array}{l}\text { INSULIN GLARGINE-- } \\
\text { IBUPROFEN--OMEPRAZOLE }\end{array}$ & 21 & 19 & 696 & 156 & 0.0014 & 0.0517 & 0.0116 & 0.0024 & 2894.88 \\
\hline 25 & $\begin{array}{l}\text { ACETAMINOPHEN-- } \\
\text { CEPHALEXIN-- } \\
\text { HYDROCODONE } \\
\text { BITARTRATE }\end{array}$ & 18 & 136 & 202 & 13 & 0.0101 & 0.0150 & 0.0010 & 0.0021 & 14333.02 \\
\hline 26 & $\begin{array}{l}\text { ACETAMINOPHEN-- } \\
\text { CIPROFLOXACIN } \\
\text { HYDROCHLORIDE-- } \\
\text { OXYCODONE } \\
\text { HYDROCHLORIDE }\end{array}$ & 18 & 136 & 100 & 140 & 0.0101 & 0.0074 & 0.0104 & 0.0021 & 2688.46 \\
\hline 27 & $\begin{array}{l}\text { ACETAMINOPHEN-- } \\
\text { CYCLOBENZAPRINE } \\
\text { HYDROCHLORIDE-- } \\
\text { OXYCODONE } \\
\text { HYDROCHLORIDE }\end{array}$ & 18 & 136 & 125 & 140 & 0.0101 & 0.0093 & 0.0104 & 0.0021 & 2150.77 \\
\hline 28 & $\begin{array}{l}\text { DIVALPROEX SODIUM-- } \\
\text { LEVETIRACETAM-- } \\
\text { OXCARBAZEPINE }\end{array}$ & 18 & 34 & 71 & 17 & 0.0025 & 0.0053 & 0.0013 & 0.0021 & 124734.07 \\
\hline 29 & $\begin{array}{l}\text { ACETAMINOPHEN-- } \\
\text { AZITHROMYCIN } \\
\text { DIHYDRATE--OXYCODONE } \\
\text { HYDROCHLORIDE }\end{array}$ & 16 & 136 & 909 & 140 & 0.0101 & 0.0675 & 0.0104 & 0.0019 & 262.90 \\
\hline 30 & $\begin{array}{l}\text { ALBUTEROL SULFATE-- } \\
\text { FLUTICASONE } \\
\text { PROPIONATE-- } \\
\text { MONTELUKAST SODIUM }\end{array}$ & 16 & 556 & 86 & 95 & 0.0413 & 0.0064 & 0.0071 & 0.0019 & 1001.66 \\
\hline
\end{tabular}


Figure 2: Relationship between active ingredient triples and occurrence and ER richness. Logarithms are in base 10.

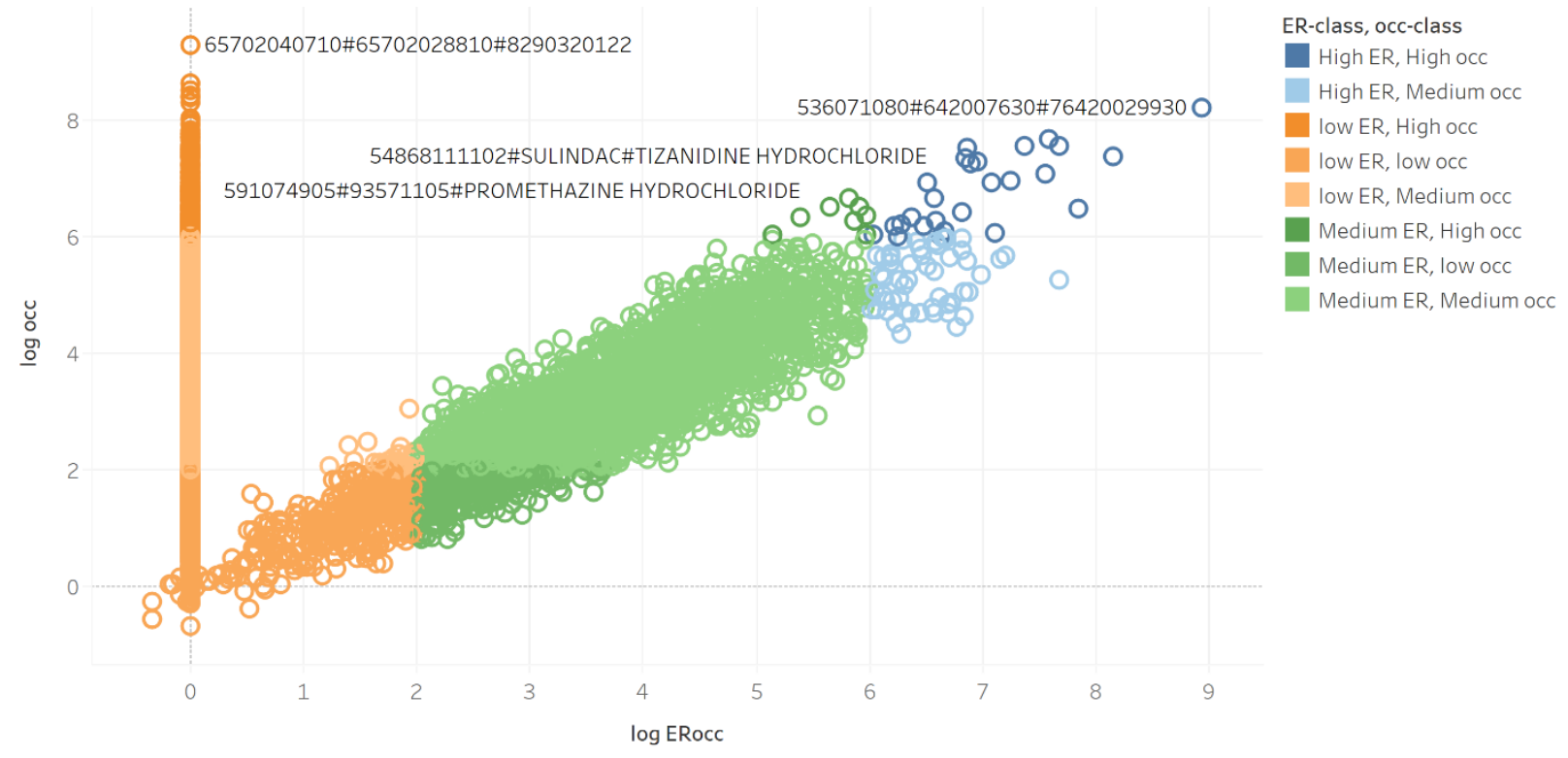

Table 17: Top 30 TC1 drug class triples and their individual and co-occurrences probability, and occurrence richness ranked by ER total.

\begin{tabular}{|c|c|c|c|c|c|c|c|c|c|c|}
\hline & TC1 drug class triples & Occ & $\begin{array}{l}\text { part1 } \\
\text {-occ }\end{array}$ & $\begin{array}{l}\text { part2 } \\
\text {-occ }\end{array}$ & $\begin{array}{l}\text { part3 } \\
\text {-occ }\end{array}$ & $\begin{array}{l}\text { p(three- } \\
\text { occ) }\end{array}$ & $\begin{array}{l}\text { p(part1- } \\
\text { occ) }\end{array}$ & $\begin{array}{l}\text { p(part2- } \\
\text { occ) }\end{array}$ & $\begin{array}{l}\text { p(part3- } \\
\text { occ) }\end{array}$ & $\begin{array}{l}\text { Occ- } \\
\text { richness }\end{array}$ \\
\hline 1 & $\begin{array}{l}\text { Cardiovascular agents-- } \\
\text { Central nervous system } \\
\text { agents-- Metabolic } \\
\text { agents }\end{array}$ & 1,376 & 10883 & 17160 & 5914 & 0.05 & 0.14 & 0.22 & 0.07 & 22.35 \\
\hline 2 & $\begin{array}{l}\text { Central nervous system } \\
\text { agents--Cardiovascular } \\
\text { agents-- Metabolic } \\
\text { agents }\end{array}$ & 1,157 & 17160 & 10883 & 5914 & 0.04 & 0.22 & 0.14 & 0.07 & 18.79 \\
\hline 3 & $\begin{array}{l}\text { Central nervous system } \\
\text { agents--Cardiovascular } \\
\text { agents-- } \\
\text { Psychotherapeutic agents }\end{array}$ & 648 & 17160 & 10883 & 3379 & 0.02 & 0.22 & 0.14 & 0.04 & 18.42 \\
\hline 4 & $\begin{array}{l}\text { Anti-infectives--Central } \\
\text { nervous system agents-- } \\
\text { Psychotherapeutic agents }\end{array}$ & 409 & 13495 & 17160 & 3379 & 0.01 & 0.17 & 0.22 & 0.04 & 9.37 \\
\hline 5 & $\begin{array}{l}\text { Anti-infectives--Central } \\
\text { nervous system agents-- } \\
\text { Topical agents }\end{array}$ & 489 & 13495 & 17160 & 6588 & 0.02 & 0.17 & 0.22 & 0.08 & 5.75 \\
\hline 6 & $\begin{array}{l}\text { Anti-infectives--Central } \\
\text { nervous system agents-- } \\
\text { Respiratory agents }\end{array}$ & 540 & 13495 & 17160 & 6407 & 0.02 & 0.17 & 0.22 & 0.08 & 6.53 \\
\hline 7 & $\begin{array}{l}\text { Anti-infectives-- } \\
\text { Hormones/hormone } \\
\text { modifiers--Respiratory } \\
\text { agents }\end{array}$ & 473 & 13495 & 8787 & 6407 & 0.02 & 0.17 & 0.11 & 0.08 & 11.17 \\
\hline
\end{tabular}




\begin{tabular}{|c|c|c|c|c|c|c|c|c|c|c|}
\hline 8 & $\begin{array}{l}\text { Cardiovascular agents-- } \\
\text { Gastrointestinal agents-- } \\
\text { Metabolic agents }\end{array}$ & 1,038 & 10883 & 2561 & 5914 & 0.04 & 0.14 & 0.03 & 0.07 & 112.95 \\
\hline 9 & $\begin{array}{l}\text { Anti-infectives--Central } \\
\text { nervous system agents-- } \\
\text { Gastrointestinal agents }\end{array}$ & 282 & 13495 & 17160 & 2561 & 0.01 & 0.17 & 0.22 & 0.03 & 8.53 \\
\hline 10 & $\begin{array}{l}\text { Anti-infectives--Central } \\
\text { nervous system agents-- } \\
\text { Hormones/hormone } \\
\text { modifiers }\end{array}$ & 439 & 13495 & 17160 & 8787 & 0.02 & 0.17 & 0.22 & 0.11 & 3.87 \\
\hline 11 & $\begin{array}{l}\text { Cardiovascular agents-- } \\
\text { Coagulation modifiers-- } \\
\text { Metabolic agents }\end{array}$ & 525 & 10883 & 332 & 5914 & 0.02 & 0.14 & 0.00 & 0.07 & 440.66 \\
\hline 12 & $\begin{array}{l}\text { Cardiovascular agents-- } \\
\text { Hormones/hormone } \\
\text { modifiers-- Metabolic } \\
\text { agents }\end{array}$ & 1,159 & 10883 & 8787 & 5914 & 0.04 & 0.14 & 0.11 & 0.07 & 36.76 \\
\hline 13 & $\begin{array}{l}\text { Central nervous system } \\
\text { agents--Gastrointestinal } \\
\text { agents-- } \\
\text { Psychotherapeutic agents }\end{array}$ & 307 & 17160 & 2561 & 3379 & 0.01 & 0.22 & 0.03 & 0.04 & 37.08 \\
\hline 14 & $\begin{array}{l}\text { Anti-infectives--Central } \\
\text { nervous system agents-- } \\
\text { Cardiovascular agents }\end{array}$ & 283 & 13495 & 17160 & 10883 & 0.01 & 0.17 & 0.22 & 0.14 & 2.01 \\
\hline 15 & $\begin{array}{l}\text { Central nervous system } \\
\text { agents-- } \\
\text { Hormones/hormone } \\
\text { modifiers-- } \\
\text { Psychotherapeutic agents }\end{array}$ & 473 & 17160 & 8787 & 3379 & 0.02 & 0.22 & 0.11 & 0.04 & 16.65 \\
\hline 16 & $\begin{array}{l}\text { Cardiovascular agents-- } \\
\text { Central nervous system } \\
\text { agents-- } \\
\text { Psychotherapeutic agents }\end{array}$ & 386 & 10883 & 17160 & 3379 & 0.01 & 0.14 & 0.22 & 0.04 & 10.97 \\
\hline 17 & $\begin{array}{l}\text { Central nervous system } \\
\text { agents-- } \\
\text { Psychotherapeutic } \\
\text { agents--Respiratory } \\
\text { agents }\end{array}$ & 271 & 17160 & 3379 & 6407 & 0.01 & 0.22 & 0.04 & 0.08 & 13.08 \\
\hline 18 & $\begin{array}{l}\text { Coagulation modifiers-- } \\
\text { Cardiovascular agents-- } \\
\text { Metabolic agents }\end{array}$ & 375 & 332 & 10883 & 5914 & 0.01 & 0.00 & 0.14 & 0.07 & 314.76 \\
\hline 19 & $\begin{array}{l}\text { Cardiovascular agents-- } \\
\text { Central nervous system } \\
\text { agents--Gastrointestinal } \\
\text { agents }\end{array}$ & 228 & 10883 & 17160 & 2561 & 0.01 & 0.14 & 0.22 & 0.03 & 8.55 \\
\hline 20 & $\begin{array}{l}\text { Anti-infectives-- } \\
\text { Respiratory agents-- } \\
\text { Topical agents }\end{array}$ & 445 & 13495 & 6407 & 6588 & 0.02 & 0.17 & 0.08 & 0.08 & 14.01 \\
\hline 21 & $\begin{array}{l}\text { Anti-infectives-- } \\
\text { Cardiovascular agents-- } \\
\text { Metabolic agents }\end{array}$ & 490 & 13495 & 10883 & 5914 & 0.02 & 0.17 & 0.14 & 0.07 & 10.12 \\
\hline 22 & $\begin{array}{l}\text { Central nervous system } \\
\text { agents--Cardiovascular } \\
\text { agents--Gastrointestinal } \\
\text { agents }\end{array}$ & 305 & 17160 & 10883 & 2561 & 0.01 & 0.22 & 0.14 & 0.03 & 11.44 \\
\hline 23 & $\begin{array}{l}\text { Cardiovascular agents-- } \\
\text { Metabolic agents -- } \\
\text { Nutritional products }\end{array}$ & 622 & 10883 & 5914 & 2258 & 0.02 & 0.14 & 0.07 & 0.03 & 76.76 \\
\hline 24 & $\begin{array}{l}\text { Central nervous system } \\
\text { agents-- Metabolic } \\
\text { agents -- } \\
\text { Psychotherapeutic agents }\end{array}$ & 319 & 17160 & 5914 & 3379 & 0.01 & 0.22 & 0.07 & 0.04 & 16.68 \\
\hline 25 & $\begin{array}{l}\text { Cardiovascular agents-- } \\
\text { Metabolic agents -- } \\
\text { Topical agents }\end{array}$ & 652 & 10883 & 5914 & 6588 & 0.02 & 0.14 & 0.07 & 0.08 & 27.58 \\
\hline
\end{tabular}




\begin{tabular}{|c|c|c|c|c|c|c|c|c|c|c|}
\hline 26 & $\begin{array}{l}\text { Cardiovascular agents-- } \\
\text { Metabolic agents -- } \\
\text { Psychotherapeutic agents }\end{array}$ & 567 & 10883 & 5914 & 3379 & 0.02 & 0.14 & 0.07 & 0.04 & 46.76 \\
\hline 27 & $\begin{array}{l}\text { Central nervous system } \\
\text { agents--Respiratory } \\
\text { agents--Topical agents }\end{array}$ & 310 & 17160 & 6407 & 6588 & 0.01 & 0.22 & 0.08 & 0.08 & 7.68 \\
\hline 28 & $\begin{array}{l}\text { Anti-infectives--Central } \\
\text { nervous system agents-- } \\
\text { Metabolic agents }\end{array}$ & 208 & 13495 & 17160 & 5914 & 0.01 & 0.17 & 0.22 & 0.07 & 2.72 \\
\hline 29 & $\begin{array}{l}\text { Central nervous system } \\
\text { agents--Gastrointestinal } \\
\text { agents-- Metabolic } \\
\text { agents }\end{array}$ & 204 & 17160 & 2561 & 5914 & 0.01 & 0.22 & 0.03 & 0.07 & 14.08 \\
\hline 30 & $\begin{array}{l}\text { Anti-infectives-- } \\
\text { Cardiovascular agents-- } \\
\text { Central nervous system } \\
\text { agents }\end{array}$ & 158 & 13495 & 10883 & 17160 & 0.01 & 0.17 & 0.14 & 0.22 & 1.12 \\
\hline
\end{tabular}

Table 18: Individual and joint probability of occurrence for the top 30 two-TC1 drug class polypharmacy (sorted by ER total) with richness of ER.

\begin{tabular}{|c|c|c|c|c|c|c|c|c|c|c|}
\hline & $\begin{array}{l}\text { TC1 drug class } \\
\text { triples }\end{array}$ & ER & $\begin{array}{l}\text { part1- } \\
\text { EROcc }\end{array}$ & $\begin{array}{l}\text { part2- } \\
\text { EROcc }\end{array}$ & $\begin{array}{l}\text { part3- } \\
\text { EROcc }\end{array}$ & $\begin{array}{l}\text { p(part1- } \\
\text { ERocc) }\end{array}$ & $\begin{array}{l}\text { p(part2- } \\
\text { ERocc) }\end{array}$ & $\begin{array}{l}\text { p(part } \\
3- \\
\text { ERocc) }\end{array}$ & $\begin{array}{l}\text { p(three- } \\
\text { case-ER) }\end{array}$ & $\begin{array}{l}\text { ER- } \\
\text { richness }\end{array}$ \\
\hline 1 & $\begin{array}{l}\text { Cardiovascular } \\
\text { agents--Central } \\
\text { nervous system } \\
\text { agents-- Metabolic } \\
\text { agents }\end{array}$ & 389 & 2245 & 6792 & 1049 & 0.104 & 0.315 & 0.049 & 0.039 & 24.55 \\
\hline 2 & $\begin{array}{l}\text { Central nervous } \\
\text { system agents-- } \\
\text { Cardiovascular } \\
\text { agents-- Metabolic } \\
\text { agents }\end{array}$ & 381 & 6792 & 2245 & 1049 & 0.315 & 0.104 & 0.049 & 0.038 & 24.05 \\
\hline 3 & $\begin{array}{l}\text { Central nervous } \\
\text { system agents-- } \\
\text { Cardiovascular } \\
\text { agents-- } \\
\text { Psychotherapeutic } \\
\text { agents }\end{array}$ & 307 & 6792 & 2245 & 831 & 0.315 & 0.104 & 0.039 & 0.031 & 24.46 \\
\hline 4 & $\begin{array}{l}\text { Anti-infectives-- } \\
\text { Central nervous } \\
\text { system agents-- } \\
\text { Psychotherapeutic } \\
\text { agents }\end{array}$ & 297 & 4053 & 6792 & 831 & 0.188 & 0.315 & 0.039 & 0.030 & 13.11 \\
\hline 5 & $\begin{array}{l}\text { Anti-infectives-- } \\
\text { Central nervous } \\
\text { system agents-- } \\
\text { Topical agents }\end{array}$ & 257 & 4053 & 6792 & 1492 & 0.188 & 0.315 & 0.069 & 0.026 & 6.32 \\
\hline 6 & $\begin{array}{l}\text { Anti-infectives-- } \\
\text { Central nervous } \\
\text { system agents-- } \\
\text { Respiratory agents }\end{array}$ & 253 & 4053 & 6792 & 1647 & 0.188 & 0.315 & 0.076 & 0.025 & 5.63 \\
\hline 7 & $\begin{array}{l}\text { Anti-infectives-- } \\
\text { Hormones/hormone } \\
\text { modifiers-- } \\
\text { Respiratory agents }\end{array}$ & 217 & 4053 & 1491 & 1647 & 0.188 & 0.069 & 0.076 & 0.022 & 22.01 \\
\hline 8 & $\begin{array}{l}\text { Cardiovascular } \\
\text { agents-- } \\
\text { Gastrointestinal } \\
\text { agents-- Metabolic } \\
\text { agents }\end{array}$ & 217 & 2245 & 714 & 1049 & 0.104 & 0.033 & 0.049 & 0.022 & 130.28 \\
\hline
\end{tabular}




\begin{tabular}{|c|c|c|c|c|c|c|c|c|c|c|}
\hline 9 & $\begin{array}{l}\text { Anti-infectives-- } \\
\text { Central nervous } \\
\text { system agents-- } \\
\text { Gastrointestinal } \\
\text { agents }\end{array}$ & 214 & 4053 & 6792 & 714 & 0.188 & 0.315 & 0.033 & 0.021 & 10.99 \\
\hline 10 & $\begin{array}{l}\text { Anti-infectives-- } \\
\text { Central nervous } \\
\text { system agents-- } \\
\text { Hormones/hormone } \\
\text { modifiers }\end{array}$ & 210 & 4053 & 6792 & 1491 & 0.188 & 0.315 & 0.069 & 0.021 & 5.16 \\
\hline 11 & $\begin{array}{l}\text { Cardiovascular } \\
\text { agents--Coagulation } \\
\text { modifiers-- } \\
\text { Metabolic agents }\end{array}$ & 197 & 2245 & 119 & 1049 & 0.104 & 0.006 & 0.049 & 0.020 & 709.61 \\
\hline 12 & $\begin{array}{l}\text { Cardiovascular } \\
\text { agents-- } \\
\text { Hormones/hormone } \\
\text { modifiers-- } \\
\text { Metabolic agents }\end{array}$ & 194 & 2245 & 1491 & 1049 & 0.104 & 0.069 & 0.049 & 0.019 & 55.77 \\
\hline 13 & $\begin{array}{l}\text { Central nervous } \\
\text { system agents-- } \\
\text { Gastrointestinal } \\
\text { agents-- } \\
\text { Psychotherapeutic } \\
\text { agents }\end{array}$ & 188 & 6792 & 714 & 831 & 0.315 & 0.033 & 0.039 & 0.019 & 47.09 \\
\hline 14 & $\begin{array}{l}\text { Anti-infectives-- } \\
\text { Central nervous } \\
\text { system agents-- } \\
\text { Cardiovascular } \\
\text { agents }\end{array}$ & 180 & 4053 & 6792 & 2245 & 0.188 & 0.315 & 0.104 & 0.018 & 2.94 \\
\hline 15 & $\begin{array}{l}\text { Central nervous } \\
\text { system agents-- } \\
\text { Hormones/hormone } \\
\text { modifiers-- } \\
\text { Psychotherapeutic } \\
\text { agents }\end{array}$ & 166 & 6792 & 1491 & 831 & 0.315 & 0.069 & 0.039 & 0.017 & 19.91 \\
\hline 16 & $\begin{array}{l}\text { Cardiovascular } \\
\text { agents--Central } \\
\text { nervous system } \\
\text { agents-- } \\
\text { Psychotherapeutic } \\
\text { agents }\end{array}$ & 148 & 2245 & 6792 & 831 & 0.104 & 0.315 & 0.039 & 0.015 & 11.79 \\
\hline 17 & $\begin{array}{l}\text { Central nervous } \\
\text { system agents-- } \\
\text { Psychotherapeutic } \\
\text { agents--Respiratory } \\
\text { agents }\end{array}$ & 147 & 6792 & 831 & 1647 & 0.315 & 0.039 & 0.076 & 0.015 & 15.96 \\
\hline 18 & $\begin{array}{l}\text { Coagulation } \\
\text { modifiers-- } \\
\text { Cardiovascular } \\
\text { agents-- Metabolic } \\
\text { agents }\end{array}$ & 143 & 119 & 2245 & 1049 & 0.006 & 0.104 & 0.049 & 0.014 & 515.10 \\
\hline 19 & $\begin{array}{l}\text { Cardiovascular } \\
\text { agents--Central } \\
\text { nervous system } \\
\text { agents-- } \\
\text { Gastrointestinal } \\
\text { agents }\end{array}$ & 138 & 2245 & 6792 & 714 & 0.104 & 0.315 & 0.033 & 0.014 & 12.80 \\
\hline 20 & $\begin{array}{l}\text { Anti-infectives-- } \\
\text { Respiratory agents-- } \\
\text { Topical agents }\end{array}$ & 130 & 4053 & 1647 & 1492 & 0.188 & 0.076 & 0.069 & 0.013 & 13.18 \\
\hline 21 & $\begin{array}{l}\text { Anti-infectives-- } \\
\text { Cardiovascular } \\
\text { agents-- Metabolic } \\
\text { agents }\end{array}$ & 128 & 4053 & 2245 & 1049 & 0.188 & 0.104 & 0.049 & 0.013 & 13.54 \\
\hline
\end{tabular}




\begin{tabular}{|c|c|c|c|c|c|c|c|c|c|c|}
\hline 22 & $\begin{array}{l}\text { Central nervous } \\
\text { system agents-- } \\
\text { Cardiovascular } \\
\text { agents-- } \\
\text { Gastrointestinal } \\
\text { agents }\end{array}$ & 121 & 6792 & 2245 & 714 & 0.315 & 0.104 & 0.033 & 0.012 & 11.22 \\
\hline 23 & $\begin{array}{l}\text { Cardiovascular } \\
\text { agents-- Metabolic } \\
\text { agents --Nutritional } \\
\text { products }\end{array}$ & 117 & 2245 & 1049 & 669 & 0.104 & 0.049 & 0.031 & 0.012 & 74.97 \\
\hline 24 & $\begin{array}{l}\text { Central nervous } \\
\text { system agents-- } \\
\text { Metabolic agents -- } \\
\text { Psychotherapeutic } \\
\text { agents }\end{array}$ & 115 & 6792 & 1049 & 831 & 0.315 & 0.049 & 0.039 & 0.012 & 19.61 \\
\hline 25 & $\begin{array}{l}\text { Cardiovascular } \\
\text { agents-- Metabolic } \\
\text { agents --Topical } \\
\text { agents }\end{array}$ & 113 & 2245 & 1049 & 1492 & 0.104 & 0.049 & 0.069 & 0.011 & 32.46 \\
\hline 26 & $\begin{array}{l}\text { Cardiovascular } \\
\text { agents-- Metabolic } \\
\text { agents -- } \\
\text { Psychotherapeutic } \\
\text { agents }\end{array}$ & 103 & 2245 & 1049 & 831 & 0.104 & 0.049 & 0.039 & 0.010 & 53.13 \\
\hline 27 & $\begin{array}{l}\text { Central nervous } \\
\text { system agents-- } \\
\text { Respiratory agents-- } \\
\text { Topical agents } \\
\end{array}$ & 103 & 6792 & 1647 & 1492 & 0.315 & 0.076 & 0.069 & 0.010 & 6.23 \\
\hline 28 & $\begin{array}{l}\text { Anti-infectives-- } \\
\text { Central nervous } \\
\text { system agents-- } \\
\text { Metabolic agents }\end{array}$ & 91 & 4053 & 6792 & 1049 & 0.188 & 0.315 & 0.049 & 0.009 & 3.18 \\
\hline 29 & $\begin{array}{l}\text { Central nervous } \\
\text { system agents-- } \\
\text { Gastrointestinal } \\
\text { agents-- Metabolic } \\
\text { agents }\end{array}$ & 90 & 6792 & 714 & 1049 & 0.315 & 0.033 & 0.049 & 0.009 & 17.86 \\
\hline 30 & $\begin{array}{l}\text { Anti-infectives-- } \\
\text { Cardiovascular } \\
\text { agents--Central } \\
\text { nervous system } \\
\text { agents }\end{array}$ & 85 & 4053 & 2245 & 6792 & 0.188 & 0.104 & 0.315 & 0.009 & 1.39 \\
\hline
\end{tabular}


Figure 3: Relationship between TC1 drug class triples and occurrence and ER richness. Logarithms are in base 10 .

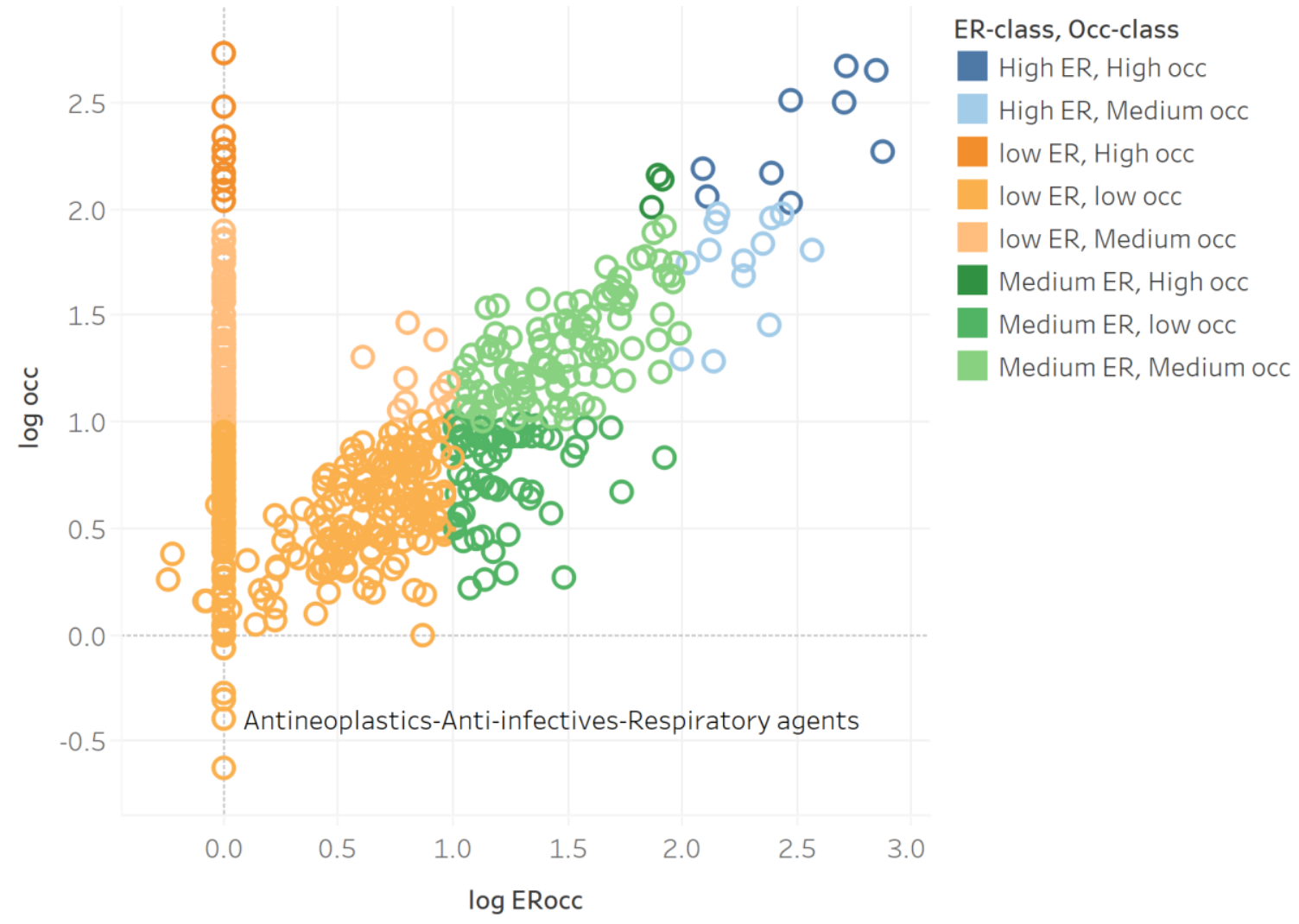

Table 19: Top 30 TC1S1 drug class triples and their individual and co-occurrences probability, and occurrence richness ranked by ER total.

\begin{tabular}{|c|c|c|c|c|c|c|c|c|c|c|}
\hline & TC1S1 drug class triples & $\begin{array}{l}\text { Oc } \\
\text { c }\end{array}$ & $\begin{array}{l}\text { part1 } \\
\text {-occ }\end{array}$ & $\begin{array}{l}\text { part2 } \\
\text {-occ }\end{array}$ & $\begin{array}{l}\text { part3 } \\
\text {-occ }\end{array}$ & $\begin{array}{l}p(\text { part1 } \\
\text {-occ) }\end{array}$ & $\begin{array}{l}p \text { (part2 } \\
-o c c)\end{array}$ & $\begin{array}{l}p \text { (part3 } \\
\text {-occ) }\end{array}$ & $\begin{array}{l}\text { p(three } \\
\text {-occ) }\end{array}$ & $\begin{array}{l}\text { Occ- } \\
\text { richnes } \\
\text { s }\end{array}$ \\
\hline 1 & $\begin{array}{l}\text { ADRENAL_CORTICAL_STEROIDS-- } \\
\text { BRONCHODILATORS-- } \\
\text { MACROLIDE_DERIVATIVES }\end{array}$ & 77 & 781 & 2683 & 3357 & 0.01 & 0.04 & 0.05 & 0.003 & 154.58 \\
\hline 2 & $\begin{array}{l}\text { ANALGESICS--ANTICONVULSANTS-- } \\
\text { MUSCLE_RELAXANTS }\end{array}$ & 66 & 8519 & 1280 & 441 & 0.12 & 0.02 & 0.01 & 0.003 & 193.82 \\
\hline 3 & $\begin{array}{l}\text { ANALGESICS--ANTIDEPRESSANTS-- } \\
\text { ANXIOLYTICSSEDATIVESAND_ } \\
\text { HYPNOTICS }\end{array}$ & 23 & 8519 & 2798 & 637 & 0.12 & 0.04 & 0.01 & 0.001 & 21.39 \\
\hline 4 & $\begin{array}{l}\text { ADRENAL_CORTICAL_STEROIDS-- } \\
\text { BRONCHODILATORS--PENICILLINS }\end{array}$ & 56 & 781 & 2683 & 5593 & 0.01 & 0.04 & 0.08 & 0.002 & 67.48 \\
\hline 5 & $\begin{array}{l}\text { ANALGESICS--PENICILLINS-- } \\
\text { UPPER_RESPIRATORY_- } \\
\text { COMBINATIONS }\end{array}$ & 66 & 8519 & 5593 & 822 & 0.12 & 0.08 & 0.01 & 0.003 & 23.80 \\
\hline 6 & $\begin{array}{l}\text { ANTIDIABETIC_AGENTS-- } \\
\text { ANGIOTENSIN_CONVERTING_ } \\
\text { ENZYME_INHIBITORS-- } \\
\text { ANTIHYPERLIPIDEMIC_AGENTS }\end{array}$ & 185 & 2079 & 2005 & 2931 & 0.03 & 0.03 & 0.04 & 0.007 & 213.83 \\
\hline 7 & $\begin{array}{l}\text { ANALGESICS--ANTICONVULSANTS- } \\
\text { ANTIDEPRESSANTS }\end{array}$ & 33 & 8519 & 1280 & 2798 & 0.12 & 0.02 & 0.04 & 0.001 & 15.27 \\
\hline
\end{tabular}




\begin{tabular}{|c|c|c|c|c|c|c|c|c|c|c|}
\hline 8 & $\begin{array}{l}\text { ANTIDIABETIC_AGENTS-- } \\
\text { ANGIOTENSIN_CONVERTING_ } \\
\text { ENZYME_INHIBITORS-- } \\
\text { BETA_ADRENERGIC_BLOCKING_- } \\
\text { AGENTS }\end{array}$ & 43 & 2079 & 2005 & 1103 & 0.03 & 0.03 & 0.02 & 0.002 & 132.07 \\
\hline 9 & $\begin{array}{l}\text { BRONCHODILATORS-- } \\
\text { LEUKOTRIENE_MODIFIERS-- } \\
\text { RESPIRATORY_INHALANT_PRODUCTS }\end{array}$ & 62 & 2683 & 457 & 200 & 0.04 & 0.01 & 0.00 & 0.002 & 3570.34 \\
\hline 10 & $\begin{array}{l}\text { ANALGESICS-IRON_PRODUCTS-- } \\
\text { LAXATIVES }\end{array}$ & 31 & 8519 & 1013 & 268 & 0.12 & 0.01 & 0.00 & 0.001 & 189.28 \\
\hline 11 & $\begin{array}{l}\text { ANALGESICS--ANTIDEPRESSANTS-- } \\
\text { MUSCLE_RELAXANTS }\end{array}$ & 46 & 8519 & 2798 & 441 & 0.12 & 0.04 & 0.01 & 0.002 & 61.80 \\
\hline 12 & $\begin{array}{l}\text { BETA_ADRENERGIC_BLOCKING_ } \\
\text { AGENTS--DIURETICS-- } \\
\text { MUSCLE_RELAXANTS }\end{array}$ & 2 & 1103 & 716 & 441 & 0.02 & 0.01 & 0.01 & 0.000 & 81.09 \\
\hline 13 & $\begin{array}{l}\text { ANALGESICS-- } \\
\text { ANTIEMETIC/ANTIVERTIGO_AGENTS- } \\
\text {-MUSCLE_RELAXANTS }\end{array}$ & 15 & 8519 & 724 & 441 & 0.12 & 0.01 & 0.01 & 0.001 & 77.88 \\
\hline 14 & $\begin{array}{l}\text { ANALGESICS-- } \\
\text { MACROLIDE_DERIVATIVES-- } \\
\text { PENICILLINS }\end{array}$ & 30 & 8519 & 3357 & 5593 & 0.12 & 0.05 & 0.08 & 0.001 & 2.65 \\
\hline 15 & $\begin{array}{l}\text { ANALGESICS--MUSCLE_RELAXANTS-- } \\
\text { PROTON_PUMP_INHIBITORS }\end{array}$ & 41 & 8519 & 441 & 1429 & 0.12 & 0.01 & 0.02 & 0.002 & 107.85 \\
\hline 16 & $\begin{array}{l}\text { ANGIOTENSIN_CONVERTING_ } \\
\text { ENZYME_INHIBITORS-- } \\
\text { BETA_ADRENERGIC_BLOCKING_ } \\
\text { AGENTS--DIURETICS } \\
\end{array}$ & 86 & 2005 & 1103 & 716 & 0.03 & 0.02 & 0.01 & 0.003 & 766.97 \\
\hline 17 & $\begin{array}{l}\text { ANALGESICS--ANTIDEPRESSANTS-- } \\
\text { ANTICONVULSANTS }\end{array}$ & 39 & 8519 & 2798 & 1280 & 0.12 & 0.04 & 0.02 & 0.001 & 18.05 \\
\hline 18 & $\begin{array}{l}\text { ANALGESICS--MUSCLE_RELAXANTS-- } \\
\text { PENICILLINS }\end{array}$ & 21 & 8519 & 441 & 5593 & 0.12 & 0.01 & 0.08 & 0.001 & 14.11 \\
\hline 19 & $\begin{array}{l}\text { ANTIDIABETIC_AGENTS-- } \\
\text { ANALGESICS-- } \\
\text { PROTON_PUMP_INHIBITORS } \\
\end{array}$ & 9 & 2079 & 8519 & 1429 & 0.03 & 0.12 & 0.02 & 0.000 & 5.02 \\
\hline 20 & $\begin{array}{l}\text { ANTIHYPERTENSIVE_COMBINATIONS } \\
\text {--BETA_ADRENERGIC_BLOCKING_- } \\
\text { AGENTS-- } \\
\text { CALCIUM_CHANNEL_BLOCKING_ } \\
\text { AGENTS }\end{array}$ & 44 & 1925 & 1103 & 804 & 0.03 & 0.02 & 0.01 & 0.002 & 363.98 \\
\hline 21 & $\begin{array}{l}\text { BETA_ADRENERGIC_BLOCKING_ } \\
\text { AGENTS-- } \\
\text { CALCIUM_CHANNEL_BLOCKING_- } \\
\text { AGENTS--DIURETICS }\end{array}$ & 40 & 1103 & 804 & 716 & 0.02 & 0.01 & 0.01 & 0.002 & 889.61 \\
\hline 22 & $\begin{array}{l}\text { ADRENAL_CORTICAL_STEROIDS-- } \\
\text { BRONCHODILATORS-- } \\
\text { RESPIRATORY_INHALANT_PRODUCTS }\end{array}$ & 29 & 781 & 2683 & 200 & 0.01 & 0.04 & 0.00 & 0.001 & 977.20 \\
\hline 23 & $\begin{array}{l}\text { ANALGESICS-- } \\
\text { ANTIEMETIC/ANTIVERTIGO_AGENTS- } \\
\text {-PENICILLINS }\end{array}$ & 24 & 8519 & 724 & 5593 & 0.12 & 0.01 & 0.08 & 0.001 & 9.82 \\
\hline 24 & $\begin{array}{l}\text { ANALGESICS--OTIC_PREPARATIONS-- } \\
\text { PENICILIINS }\end{array}$ & 21 & 8519 & 350 & 5593 & 0.12 & 0.00 & 0.08 & 0.001 & 17.78 \\
\hline 25 & $\begin{array}{l}\text { ANALGESICS-- } \\
\text { ANTIEMETIC/ANTIVERTIGO_AGENTS- } \\
\text {-MACROLIDE_DERIVATIVES }\end{array}$ & 17 & 8519 & 724 & 3357 & 0.12 & 0.01 & 0.05 & 0.001 & 11.59 \\
\hline 26 & $\begin{array}{l}\text { ANALGESICS--BRONCHODILATORS-- } \\
\text { RESPIRATORY_INHALANT_PRODUCTS }\end{array}$ & 22 & 8519 & 2683 & 200 & 0.12 & 0.04 & 0.00 & 0.001 & 67.96 \\
\hline 27 & $\begin{array}{l}\text { ANALGESICS--CEPHALOSPORINS-- } \\
\text { MISCELLANEOUS_ANTIBIOTICS }\end{array}$ & 16 & 8519 & 871 & 544 & 0.12 & 0.01 & 0.01 & 0.001 & 55.98 \\
\hline 28 & $\begin{array}{l}\text { ANTICOAGULANTS-- } \\
\text { ANTIARRHYTHMIC_AGENTS-- } \\
\text { BETA_ADRENERGIC_BLOCKING_ } \\
\text { AGENTS }\end{array}$ & 15 & 256 & 398 & 1103 & 0.00 & 0.01 & 0.02 & 0.001 & 1884.85 \\
\hline
\end{tabular}




\begin{tabular}{|c|l|c|c|c|c|c|c|c|c|c|}
\hline 29 & $\begin{array}{l}\text { ADRENAL_CORTICAL_STEROIDS-- } \\
\text { BRONCHODILATORS--QUINOLONES }\end{array}$ & 15 & 781 & 2683 & 393 & 0.01 & 0.04 & 0.01 & 0.001 & 257.22 \\
\hline 30 & $\begin{array}{l}\text { ANALGESICS--IRON_PRODUCTS-- } \\
\text { VITAMINS }\end{array}$ & 16 & 8519 & 1013 & 483 & 0.12 & 0.01 & 0.01 & 0.001 & 54.21 \\
\hline
\end{tabular}

Table 20: Individual and joint probability of occurrence for the top 30 two-TC1S1 drug class polypharmacy (sorted by ER total) with richness of and ER.

\begin{tabular}{|c|c|c|c|c|c|c|c|c|c|c|}
\hline & $\begin{array}{l}\text { TC1S1 drug class } \\
\text { triples }\end{array}$ & ER & $\begin{array}{l}\text { part1- } \\
\text { EROcc }\end{array}$ & $\begin{array}{l}\text { part2- } \\
\text { EROcc }\end{array}$ & $\begin{array}{l}\text { part3- } \\
\text { EROcc }\end{array}$ & $\begin{array}{l}\text { p(part1- } \\
\text { ERocc) }\end{array}$ & $\begin{array}{l}\text { p(part2- } \\
\text { ERocc) }\end{array}$ & $\begin{array}{l}\text { p(part } \\
3- \\
\text { ERocc) }\end{array}$ & $\begin{array}{l}\text { p(three- } \\
\text { case-ER) }\end{array}$ & $\begin{array}{l}\text { ER- } \\
\text { richness }\end{array}$ \\
\hline 1 & $\begin{array}{l}\text { ADRENAL_CORTICAL_ } \\
\text { STEROIDS-- } \\
\text { BRONCHODILATORS-- } \\
\text { MACROLIDE_- } \\
\text { DERIVATIVES } \\
\end{array}$ & 52 & 302 & 723 & 932 & 0.02 & 0.04 & 0.05 & 0.01 & 160.16 \\
\hline 2 & $\begin{array}{l}\text { ANALGESICS-- } \\
\text { ANTICONVULSANTS-- } \\
\text { MUSCLE_RELAXANTS }\end{array}$ & 45 & 3973 & 505 & 166 & 0.23 & 0.03 & 0.01 & 0.01 & 84.69 \\
\hline 3 & $\begin{array}{l}\text { ANALGESICS-- } \\
\text { ANTIDEPRESSANTS-- } \\
\text { ANXIOLYTICSSEDATIVES } \\
\text { AND_HYPNOTICS }\end{array}$ & 43 & 3973 & 575 & 160 & 0.23 & 0.03 & 0.01 & 0.01 & 73.74 \\
\hline 4 & $\begin{array}{l}\text { ADRENAL_- } \\
\text { CORTICAL_STEROIDS-- } \\
\text { BRONCHODILATORS-- } \\
\text { PENICILLINS }\end{array}$ & 37 & 302 & 723 & 1591 & 0.02 & 0.04 & 0.09 & 0.00 & 66.76 \\
\hline 5 & $\begin{array}{l}\text { ANALGESICS-- } \\
\text { PENICILLINS-- } \\
\text { UPPER_RESPIRATORY_- } \\
\text { COMBINATIONS } \\
\end{array}$ & 30 & 3973 & 1591 & 150 & 0.23 & 0.09 & 0.01 & 0.00 & 19.83 \\
\hline 6 & $\begin{array}{l}\text { ANTIDIABETIC_AGENTS } \\
-- \\
\text { ANGIOTENSIN_CONVER } \\
\text { TING_ENZYME_ } \\
\text { INHIBITORS-- } \\
\text { ANTIHYPERLIPIDEMIC_ } \\
\text { AGENTS }\end{array}$ & 29 & 406 & 329 & 282 & 0.02 & 0.02 & 0.02 & 0.00 & 482.55 \\
\hline 7 & $\begin{array}{l}\text { ANALGESICS-- } \\
\text { ANTICONVULSANTS-- } \\
\text { ANTIDEPRESSANTS }\end{array}$ & 28 & 3973 & 505 & 575 & 0.23 & 0.03 & 0.03 & 0.00 & 15.21 \\
\hline 8 & $\begin{array}{l}\text { ANTIDIABETIC_AGENTS } \\
-- \\
\text { ANGIOTENSIN_CONVER } \\
\text { TING_ENZYME } \\
\text { _INHIBITORS-- } \\
\text { BETA_ADRENERGIC_- } \\
\text { BLOCKING_AGENTS }\end{array}$ & 26 & 406 & 329 & 196 & 0.02 & 0.02 & 0.01 & 0.00 & 622.46 \\
\hline 9 & $\begin{array}{l}\text { BRONCHODILATORS-- } \\
\text { LEUKOTRIENE_- } \\
\text { MODIFIERS-- } \\
\text { RESPIRATORY_- } \\
\text { INHALANT_PRODUCTS } \\
\end{array}$ & 26 & 723 & 97 & 63 & 0.04 & 0.01 & 0.00 & 0.00 & 3688.42 \\
\hline 10 & $\begin{array}{l}\text { ANALGESICS-- } \\
\text { IRON_PRODUCTS-- } \\
\text { LAXATIVES }\end{array}$ & 25 & 3973 & 394 & 91 & 0.23 & 0.02 & 0.01 & 0.00 & 110.00 \\
\hline 11 & $\begin{array}{l}\text { ANALGESICS-- } \\
\text { ANTIDEPRESSANTS-- } \\
\text { MUSCLE_RELAXANTS }\end{array}$ & 24 & 3973 & 575 & 166 & 0.23 & 0.03 & 0.01 & 0.00 & 39.67 \\
\hline 12 & $\begin{array}{l}\text { BETA_ADRENERGIC_- } \\
\text { BLOCKING_AGENTS-- } \\
\text { DIURETICS-- } \\
\text { MUSCLE_RELAXANTS }\end{array}$ & 24 & 196 & 164 & 166 & 0.01 & 0.01 & 0.01 & 0.00 & 2819.17 \\
\hline 13 & $\begin{array}{l}\text { ANALGESICS-- } \\
\text { ANTIEMETIC/ANTIVERTI } \\
\text { GO_AGENTS-- } \\
\text { MUSCLE_RELAXANTS }\end{array}$ & 23 & 3973 & 420 & 166 & 0.23 & 0.02 & 0.01 & 0.00 & 52.04 \\
\hline
\end{tabular}




\begin{tabular}{|c|c|c|c|c|c|c|c|c|c|c|}
\hline 14 & $\begin{array}{l}\text { ANALGESICS-- } \\
\text { MACROLIDE-- } \\
\text { DERIVATIVES-- } \\
\text { PENICILLINS }\end{array}$ & 23 & 3973 & 932 & 1591 & 0.23 & 0.05 & 0.09 & 0.00 & 2.45 \\
\hline 15 & $\begin{array}{l}\text { ANALGESICS-- } \\
\text { MUSCLE_RELAXANTS-- } \\
\text { PROTON_PUMP_- } \\
\text { INHIBITORS } \\
\end{array}$ & 23 & 3973 & 166 & 315 & 0.23 & 0.01 & 0.02 & 0.00 & 69.39 \\
\hline 16 & $\begin{array}{l}\text { ANGIOTENSIN_CONVER } \\
\text { TING_ENZYME_- } \\
\text { INHIBITORS-- } \\
\text { BETA_ADRENERGIC_- } \\
\text { BLOCKING_AGENTS-- } \\
\text { DIURETICS }\end{array}$ & 23 & 329 & 196 & 164 & 0.02 & 0.01 & 0.01 & 0.00 & 1363.17 \\
\hline 17 & $\begin{array}{l}\text { ANALGESICS-- } \\
\text { ANTIDEPRESSANTS-- } \\
\text { ANTICONVULSANTS } \\
\end{array}$ & 22 & 3973 & 575 & 505 & 0.23 & 0.03 & 0.03 & 0.00 & 11.95 \\
\hline 18 & $\begin{array}{l}\text { ANALGESICS-- } \\
\text { MUSCLE_RELAXANTS-- } \\
\text { PENICILLINS } \\
\end{array}$ & 22 & 3973 & 166 & 1591 & 0.23 & 0.01 & 0.09 & 0.00 & 13.14 \\
\hline 19 & $\begin{array}{l}\text { ANTIDIABETIC_AGENTS- } \\
\text {-ANALGESICS-- } \\
\text { PROTON_PUMP_ } \\
\text { INHIBITORS } \\
\end{array}$ & 21 & 406 & 3973 & 315 & 0.02 & 0.23 & 0.02 & 0.00 & 25.90 \\
\hline 20 & $\begin{array}{l}\text { ANTIHYPERTENSIVE- } \\
\text { COMBINATIONS-- } \\
\text { BETA_ADRENERGIC- } \\
\text { BLOCKING_AGENTS-- } \\
\text { CALCIUM_CHANNEL_- } \\
\text { BLOCKING_AGENTS }\end{array}$ & 21 & 242 & 196 & 114 & 0.01 & 0.01 & 0.01 & 0.00 & 2434.22 \\
\hline 21 & $\begin{array}{l}\text { BETA_ADRENERGIC_- } \\
\text { BLOCKING_AGENTS-- } \\
\text { CALCIUM_CHANNEL- } \\
\text { BLOCKING_AGENTS-- } \\
\text { DIURETICS }\end{array}$ & 21 & 196 & 114 & 164 & 0.01 & 0.01 & 0.01 & 0.00 & 3591.96 \\
\hline 22 & $\begin{array}{l}\text { ADRENAL_CORTICAL_- } \\
\text { STEROIDS-- } \\
\text { BRONCHODILATORS-- } \\
\text { RESPIRATORY_- } \\
\text { INHALANT_PRODUCTS }\end{array}$ & 20 & 302 & 723 & 63 & 0.02 & 0.04 & 0.00 & 0.00 & 911.30 \\
\hline 23 & $\begin{array}{l}\text { ANALGESICS-- } \\
\text { ANTIEMETIC/ANTIVERTI } \\
\text { GO_AGENTS-- } \\
\text { PENICILIINS }\end{array}$ & 20 & 3973 & 420 & 1591 & 0.23 & 0.02 & 0.09 & 0.00 & 4.72 \\
\hline 24 & $\begin{array}{l}\text { ANALGESICS-- } \\
\text { OTIC_PREPARATIONS-- } \\
\text { PENICILLINS } \\
\end{array}$ & 20 & 3973 & 86 & 1591 & 0.23 & 0.00 & 0.09 & 0.00 & 23.06 \\
\hline 25 & $\begin{array}{l}\text { ANALGESICS-- } \\
\text { ANTIEMETIC/ANTIVERTI } \\
\text { GO_AGENTS-- } \\
\text { MACROLIIE_- } \\
\text { DERIVATIVES }\end{array}$ & 19 & 3973 & 420 & 932 & 0.23 & 0.02 & 0.05 & 0.00 & 7.66 \\
\hline 26 & $\begin{array}{l}\text { ANALGESICS-- } \\
\text { BRONCHODILATORS-- } \\
\text { RESPIRATORY_ } \\
\text { INHALANT_PRODUCTS }\end{array}$ & 19 & 3973 & 723 & 63 & 0.23 & 0.04 & 0.00 & 0.00 & 65.81 \\
\hline 27 & $\begin{array}{l}\text { ANALGESICS-- } \\
\text { CEPHALOSPORINS-- } \\
\text { MISCELLANEOUS_- } \\
\text { ANTIBIOTICS }\end{array}$ & 19 & 3973 & 290 & 203 & 0.23 & 0.02 & 0.01 & 0.00 & 50.92 \\
\hline 28 & $\begin{array}{l}\text { ANTICOAGULANTS-- } \\
\text { ANTIARRHYTHMIC_- } \\
\text { AGENTS-- } \\
\text { BETA_ADRENERGIC_- } \\
\text { BLOCKING_AGENTS }\end{array}$ & 19 & 102 & 77 & 196 & 0.01 & 0.00 & 0.01 & 0.00 & 7736.14 \\
\hline 29 & $\begin{array}{l}\text { ADRENAL_CORTICAL_- } \\
\text { STEROIDS-- } \\
\text { BRONCHODILATORS-- } \\
\text { QUINOLONES } \\
\end{array}$ & 17 & 302 & 723 & 141 & 0.02 & 0.04 & 0.01 & 0.00 & 346.10 \\
\hline 30 & $\begin{array}{l}\text { ANALGESICS-- } \\
\text { IRON_PRODUCTS-- } \\
\text { VITAMINS }\end{array}$ & 17 & 3973 & 394 & 89 & 0.23 & 0.02 & 0.01 & 0.00 & 76.48 \\
\hline
\end{tabular}


Figure 4: Relationship between TC1S1 drug class triples and occurrence and ER richness. Logarithms are in base 10.

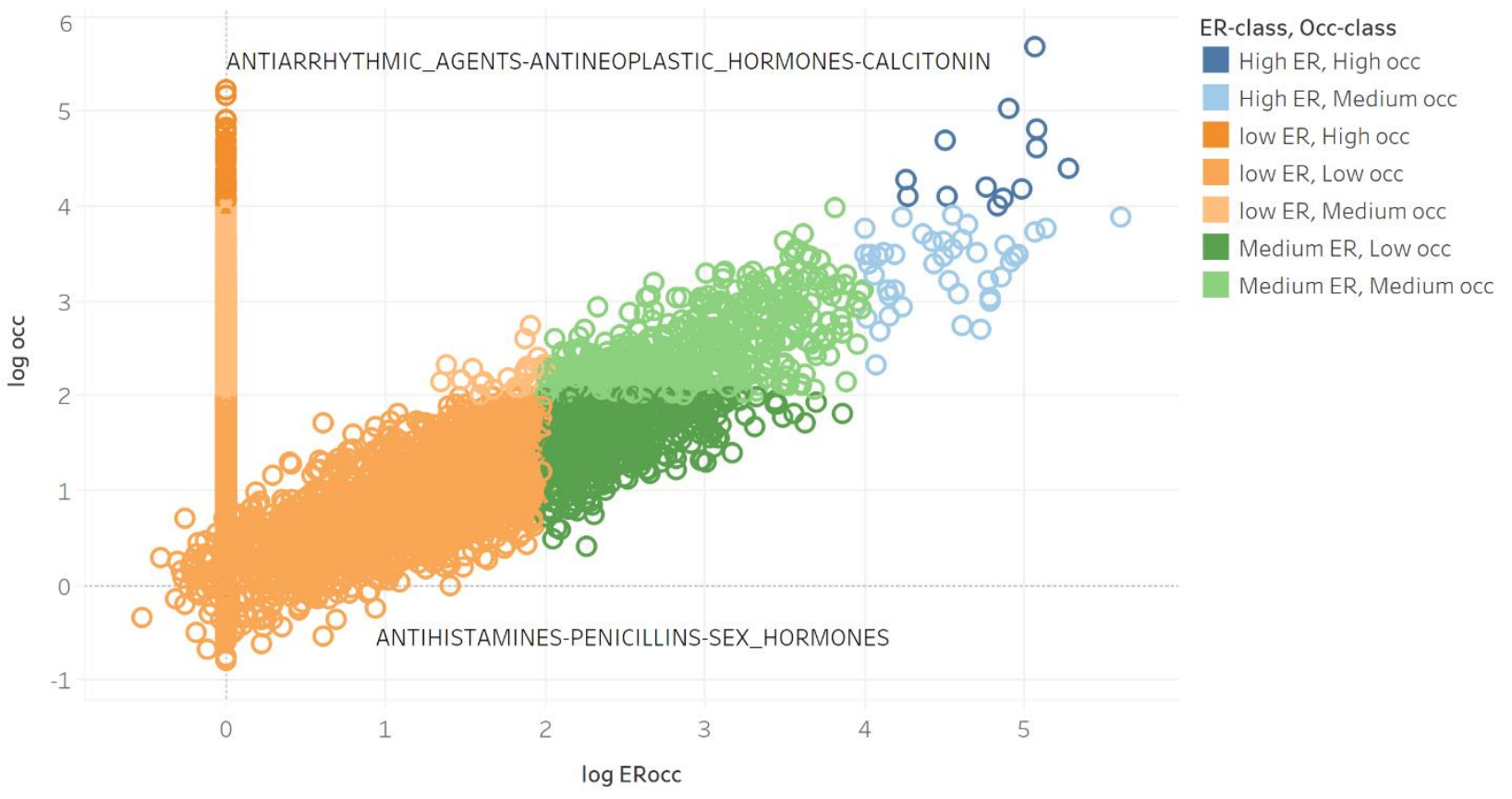

